\title{
On the feasibility of integrated optical waveguide-based in situ monitoring of microelectromechanical systems (MEMS)
}

\author{
Kolin Shay Brown \\ West Virginia University
}

Follow this and additional works at: https://researchrepository.wvu.edu/etd

\section{Recommended Citation}

Brown, Kolin Shay, "On the feasibility of integrated optical waveguide-based in situ monitoring of microelectromechanical systems (MEMS)" (2000). Graduate Theses, Dissertations, and Problem Reports. 1221.

https://researchrepository.wvu.edu/etd/1221

This Dissertation is protected by copyright and/or related rights. It has been brought to you by the The Research Repository @ WVU with permission from the rights-holder(s). You are free to use this Dissertation in any way that is permitted by the copyright and related rights legislation that applies to your use. For other uses you must obtain permission from the rights-holder(s) directly, unless additional rights are indicated by a Creative Commons license in the record and/ or on the work itself. This Dissertation has been accepted for inclusion in WVU Graduate Theses, Dissertations, and Problem Reports collection by an authorized administrator of The Research Repository @ WVU.

For more information, please contact researchrepository@mail.wvu.edu. 


\title{
On the Feasibility of Integrated Optical Waveguide- based In-Situ Monitoring of Microelectromechanical Systems (MEMS)
}

\author{
by \\ Kolin Shay Brown
}

Dissertation submitted to The College of Engineering and Mineral Resources

at

WEST VIRGINIA UNIVERSITY

in partial fulfillment of the requirements for the degree of

Doctor of Philosophy in Engineering

Lawrence A. Hornak, Ph.D., Chair

Biswajit Das, Ph.D.

Parvis Famouri, Ph.D.

Mark Jerabek, Ph.D.

Charter Stienspring, Ph.D.

Department of Computer Science and Electrical Engineering

Morgantown, West Virginia

2000 


\section{ABSTRACT \\ On the Feasibility of Integrated Optical Waveguide-based In-Situ Monitoring of Microelectromechanical Systems (MEMS)}

\section{Kolin Shay Brown}

This dissertation explores the feasibility of using integrated optical waveguides to measure the motion of microelectromechanical structures (MEMS). MEMS are a class of silicon devices which are being developed as sensors and actuators. Because these free moving structures are fabricated using processes similar to microfabrication, MEMS devices and traditional electronics can be integrated on the same substrate. This merging of the technologies will allow the miniaturization of large scale mechanical systems. A difficulty with MEMS devices is determining the submicron motion. One method of noninvasive measurement is optical measurement. Research focused on the characterization of one particular MEMS device, a linear comb resonator. Linear comb resonators displace linearly along a single axis when drive with a sinusoidal voltage signal. This research presents how single mode and multimode guided waves have potential to yield significant positional information. Using optical fibers to create a bulk optical metrology probe, the displacement and operating frequency of this device was characterized. Integration of this an optical probe structure with the MEMS devices can create integrated optical metrology (IOM), which is an in-situ method of device characterization and can represent an enabling technology for MEMS. Co-integration of the two technologies can be achieved through either processing or post processing of integrated waveguides with the MEMS devices. The fabrication process for co-integration of polymer optical waveguides has been experimentally defined in this dissertation, however final results indicate guides wave IOM would best be explored through process interruption or hybrid techniques given existing polymer materials. Analysis yields that the cointegration of inorganic waveguide structures first requires optimization of the design of the microprobe layout. 


\section{Acknowledgements}

I would like to thank Dr. Larry Hornak, my advisor, for his guidance, instruction, and encouragement throughout this research and graduate school. I would also like to thank my committee members, Dr. Biswajit Das, Dr. Parvis Famouri, Dr. Mark Jerabek, and Dr. Charter Stienspring for their suggestions and assistance. An expression of gratitude goes to Jeremy Dawson for his assistance in this research and my fellow students, Steve McGinnis and Paul Sines, for thier help.

I would like to thank my parents, whose continuous support allowed me to pursue my degree, and finally I would like to thank God, my Lord and Savior, who makes all things possible. 


\section{Table of Contents}

$\begin{array}{ll}\text { Abstract } & \text { ii }\end{array}$

Acknowledgments

$\begin{array}{ll}\text { List of Figures } & \text { ix }\end{array}$

1 Introduction $\quad 1$

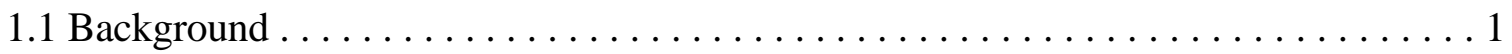

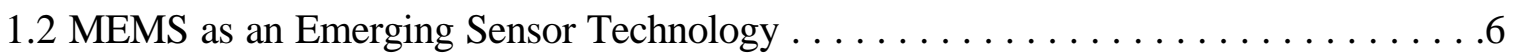

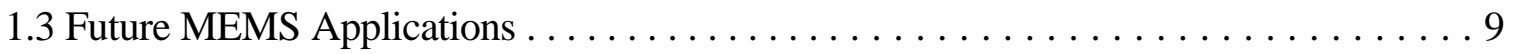

1.4 Monitoring as a MEMS Enabling Technology $\ldots \ldots \ldots \ldots \ldots \ldots \ldots \ldots \ldots \ldots$

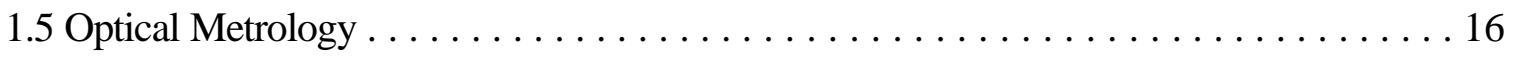

$2 \quad$ Research Motivation and Outline $\quad 17$

2.1 Current Micromachined Optic Devices . . . . . . . . . . . . . . . . 17

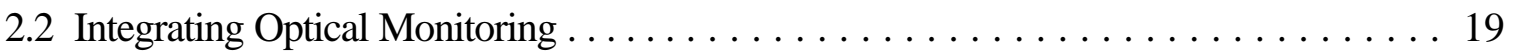

2.3 Development of Integrated Optical Monitoring Cells $\ldots \ldots \ldots \ldots \ldots \ldots \ldots \ldots$

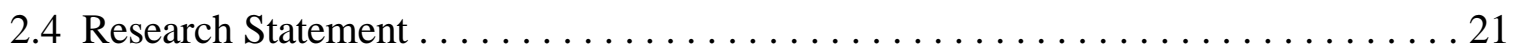

2.4.1 Study of the optical waveguides and signal intensity. . . . . . . 23

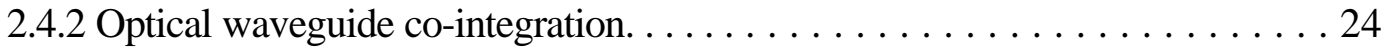




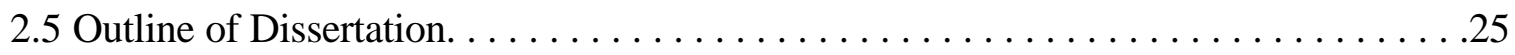

$3 \quad$ Theoretical Calculations 2

3.1 Microelectromechanical Devices . . . . . . . . . . . . . . . . . . . . . . 29

3.2 Linear Comb Resonator . . . . . . . . . . . . . . . . . . . . . . . . . . . . . . . . . . . . 30

3.3 Linear Comb Resonator Operation ............................. 34

3.4 Linear Resonator Forces Under Ideal Operation . . . . . . . . . . . . . . . . . . . 36

3.4.1 Electro static Comb Forces . . . . . . . . . . . . . . . . . . . 37

3.4.2 Folded Flexure Spring Forces $\ldots \ldots \ldots \ldots \ldots \ldots \ldots \ldots \ldots \ldots \ldots \ldots \ldots \ldots \ldots$

3.4.3 Air Resistive Damping Force $\ldots \ldots \ldots \ldots \ldots \ldots \ldots \ldots \ldots \ldots \ldots$

3.5 Model of Linear Resonator Motion $\ldots \ldots \ldots \ldots \ldots \ldots \ldots \ldots \ldots \ldots \ldots$

3.6 Resonant Frequency Calculation ........................... 48

3.7 Calculated Frequency for a 550 $\mathrm{mm}$ Flexure Length Linear Resonator. . . . . . . . . . . 49

$4 \quad$ Bulk Optical Metrology $\quad 53$

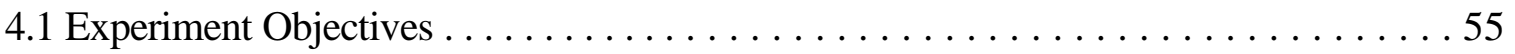

4.2 Bulk Optical Metrology Experimental Setup .....................56

4.3 Optical Measurement of Displacement of a Large Reflective Surface. . . . . . . . . . 57

4.4 Measuring the Displacement of a Linear Resonator Stage. . . . . . . . . . . . . . .62

4.5 Forced Linear Resonator Displacement Measurement. ..................... 64

4.6 Optical Measurement of Static Electrostatic Deflection ............... 67

4.7 Sinusoidal Actuated Linear Resonator Feedback Signal . . . . . . . . . . . . . . . .72

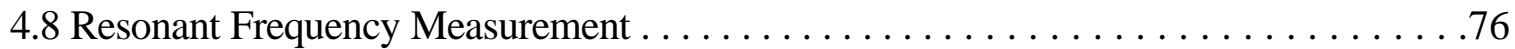


4.9 Fiber Alignment Position Relation to Output Waveform . . . . . . . . . . . . 78

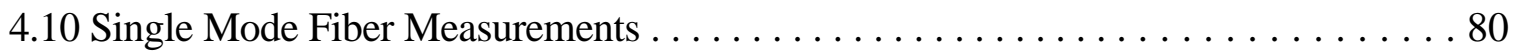

4.11 Displacement Measurements Using a Single Mode Input Fiber. . . . . . . . . . . 81

4.12 Sinusoidal Actuated Linear Resonator Feedback Signal Using a Single Mode Input

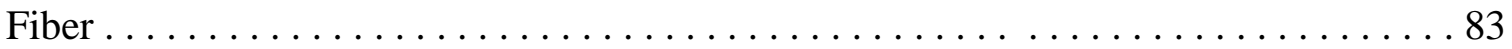

4.13 Multimode and Single Mode Measurements . . . . . . . . . . . . . 88

$5 \quad$ Integrated Optical Metrology $\quad 91$

5.1 Bulk Optical Metrology Review $\ldots \ldots \ldots \ldots \ldots \ldots \ldots \ldots \ldots \ldots \ldots \ldots \ldots \ldots$

5.2 Integrating Optical Waveguides with MEMS Devices $\ldots \ldots \ldots \ldots \ldots \ldots \ldots$

5.3 Critical Design Issues for the Co-Integration of Waveguide with MEMS Devices. . . 95

5.3 .1 Optical Waveguide Geometry . . . . . . . . . . . . . . . . . . . 96

5.3 .2 Optical Waveguide Fabrication $\ldots \ldots \ldots \ldots \ldots \ldots \ldots \ldots \ldots$

5.4 Optical Waveguide Material Selection . . . . . . . . . . . . . . . . . 101

5.5 Inorganic Optical Materials . . . . . . . . . . . . . . . . . . . . . 102

5.6 Co-integrated Fabrication Process Utilizing Inorganic Waveguides. . . . . . . . . . . 105

5.7 Organic Optical Materials . . . . . . . . . . . . . . . . . . . . . . . 108

5.8 Optical Polymer Characteristics . . . . . . . . . . . . . . . . . . . . 109

5.9 Polymer Waveguide Co-Integration $\ldots \ldots \ldots \ldots \ldots \ldots \ldots \ldots \ldots \ldots \ldots \ldots \ldots \ldots$

5.10 Inorganic and Organic Waveguide Applications . . . . . . . . . . . . . . 115

$6 \quad$ Inorganic Single Mode Waveguides $\quad 116$

6.1 Primary Mode Profile . . . . . . . . . . . . . . . . . . . . . . . 117 
6.2 Single Mode Optical Waveguide Layout for Integrated Optical Metrology. . . . . . . 123

6.3 Optical Measurement of Linear Resonator Motion . . . . . . . . . . . . . . . 125

6.4 Optical Interference Measurement $\ldots \ldots \ldots \ldots \ldots \ldots \ldots \ldots \ldots \ldots \ldots \ldots \ldots \ldots$

6.5 Single Mode Integrated Optical Metrology . . . . . . . . . . . . . . 133

$7 \quad$ Multimode Polymer Waveguides $\quad 134$

7.1 Desired Optical Polymer Characteristics for the Formation of Co-Integrated Optical

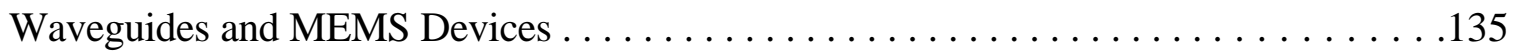

7.2 Ultradel 9000 Polymers . . . . . . . . . . . . . . . . . . . 136

7.3 Waveguide Cross-Sectional Structure . . . . . . . . . . . . . . . . . 137

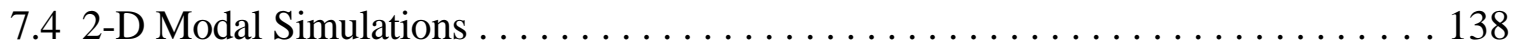

7.5 Single Mode Analysis . . . . . . . . . . . . . . . . . . . . . . . . . . . . 143

7.6 Waveguide Layout $\ldots \ldots \ldots \ldots \ldots \ldots \ldots \ldots \ldots \ldots \ldots \ldots \ldots \ldots \ldots \ldots$

7.7 Simulated Optical Measurement of Linear Resonator Displacement. . . . . . . . . . . 146

$8 \quad$ Optical Waveguide Co-Integration $\quad 157$

8.1 Ultradel Optical Polymer Waveguides . . . . . . . . . . . . . . . . . . . . . . . 157

8.2 Waveguide Mask Layout . . . . . . . . . . . . . . . . . . . . . . . 159

8.3 Ultradel Waveguide Process Flow $\ldots \ldots \ldots \ldots \ldots \ldots \ldots \ldots \ldots \ldots \ldots \ldots \ldots \ldots$

8.4 Fabricated Ultradel Polymer Waveguides . . . . . . . . . . . . . . . . . . . 167

8.5 Prefabricated Linear Resonator . . . . . . . . . . . . . . . . . . . . . . 169

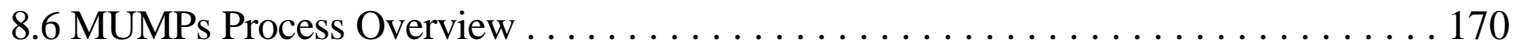

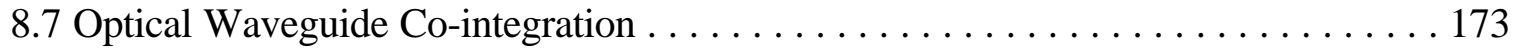

vii 
8.7.1 Optical Waveguide Co-integration Masks . . . . . . . . . . . . . . . . 173

8.7.2 Ultradel Waveguide Co-Integration . . . . . . . . . . . . . . . . . . 174

8.7 .3 Results . . . . . . . . . . . . . . . . . . . . . . .

8.8 Future Work . . . . . . . . . . . . . . . . . . . . . . . . . . . . 182

$9 \quad$ Summary and Conclusions $\quad 184$

$\begin{array}{ll}\text { Bibliography } & 187\end{array}$

$\begin{array}{ll}\text { Vita } & 194\end{array}$ 


\section{List of Figures}

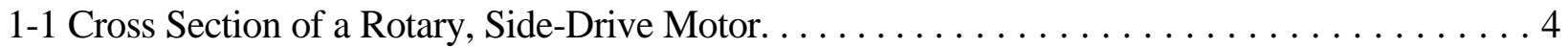

1-2 Process Outline, with Motor Cross-Section, for MCNC MUMPs Surface Micro-Machining

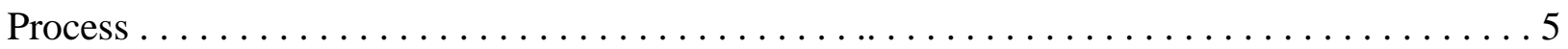

1-3 Predicted Development of MEMS Devices $\ldots \ldots \ldots \ldots \ldots \ldots \ldots \ldots \ldots \ldots \ldots$

1-4 Example MEMS Devices . . . . . . . . . . . . . . . . . . . . . . . . . 14

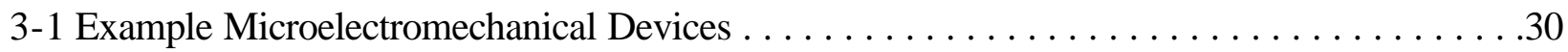

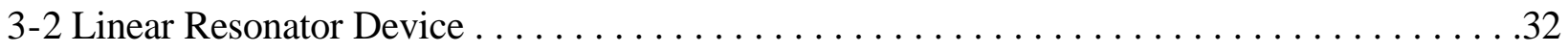

3-3 MEMS Figure Layout Emphasizing a.) Ground Plane, b.) Resonator Stage, c.) Folded Flexures, and

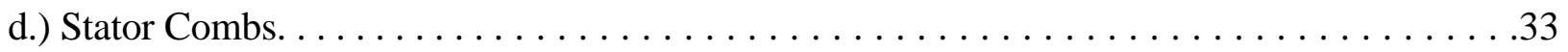

3-4 Typical Operation of a Linear Resonator MEMS Device. . . . . . . . . . . . . . . . . 36

3-5 Dynamic Model of Lumped Force on Linear Resonator Device. . . . . . . . . . . . . . .37

3-6 Close up of Resonant CombDrive Structure . . . . . . . . . . . . . . . . . 38

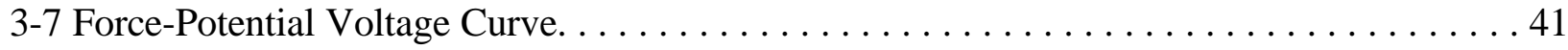

3-8 Frequency Plot of Amplitude of 550 Fm Flexure Length Linear Resonator. . . . . . . . . . .52

3-9 Frequency Plot of Phase Shift of 550 Fm Flexure Length Linear Resonator. . . . . . . . . .52

4-1 Fiber Optic Probe Setup for Bulk Optical Metrology. . . . . . . . . . . . . . . . . . . 57

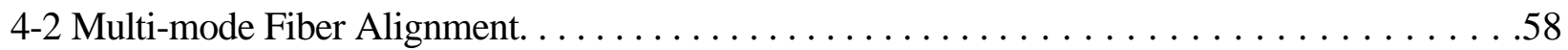


4-3 Position of Digital Linear Gauge. . . . . . . . . . . . . . . . . . . . . . . . . .59

4-4 Optical Measurement of Displacement Using a Multimode Input Fiber and Multimode Detector

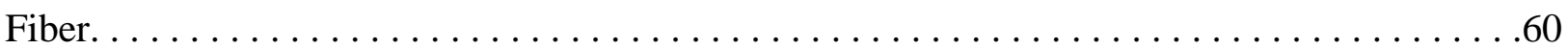

4-5 Optical Displacement Measurement of Large Reflecting Surface. . . . . . . . . . . . . 61

4-6 Experimental Setup for Measuring Linear Resonator Displacement and Motion. . . . . . . .62

4-7 Fiber and Probe Alignments for the Multimode Fiber Forced Displacement Measurement. .65

4-8 Zero Reference of Linear Resonator Position. . . . . . . . . . . . . . . . . . . 68

4-9 Forced Static Displacement Versus the Output Detector Signal Voltage. . . . . . . . . . . . 69

4-10 Input and Detector Fiber Alignment for Electrostatic Actuated Measurements. . . . . . . . 69

4-11 Measured Deflection Using DC Electrostatic Displacement of the Linear Resonator Stage. 70

4-12 Predicted Low Frequency Output Waveform. ......................... 71

4-13 $525 \mathrm{KHz}$ Linear Resonator Motion Measurement. . . . . . . . . . . . . . . . . . . . . 72

$4-141.5 \mathrm{KHz}$ Linear Resonator Motion Measurement......................... 73

4-15 $1.575 \mathrm{KHz}$ Linear Resonator Measurement. .............................73

4-16 $2.05 \mathrm{KHz}$ Linear Resonator Motion Measurement. . . . . . . . . . . . . . . . . . . . . . . 74

4-17 $2.625 \mathrm{KHz}$ Linear Resonator Motion Measurement. ........................ . 74

4-18 Resonant Frequency Measurement. .............................. 77

4-19 Multimode Input Fiber Alignment1 and the Oscilloscope Curve Trace of the Resulting Output

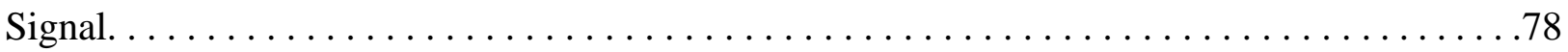

4-20 Multimode Input Fiber Alignment 2 and the Oscilloscope Curve Trace of the Resulting Output

Signal. ..................................................... 79 
4-21 Multimode Input Fiber Alignment 3 and the Oscilloscope Curve Trace of the Resulting Output

Signal. . . . . . . . . . . . . . . . . . . . . 79

4-22 Multimode Input Fiber Alignment 4 and the Oscilloscope Curve Trace of the Resulting Output

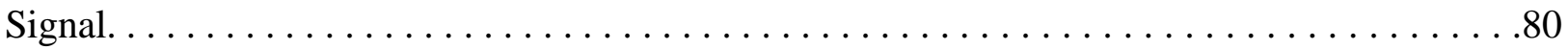

4-23 Measured Optical Displacement of a Reflecting Surface Using a Single Mode Input Fiber and

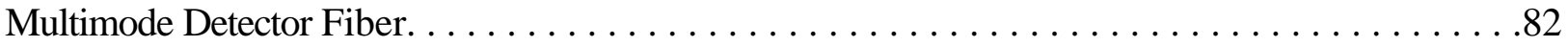

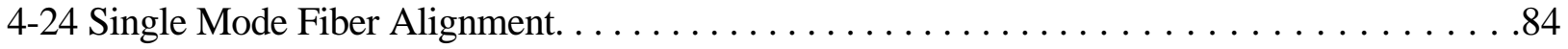

4-25 462Hz Linear Resonator Motion Measurement . . . . . . . . . . . . . . . . . 86

4-26 925Hz Linear Resonator Motion Measurement. . . . . . . . . . . . . . . . . . . . . . . . . .86

4-27 1.387KHz Linear Resonator Motion Measurement. . . . . . . . . . . . . . . . . . . . . . .87

4-28 1.85KHz Linear Resonator Motion Measurement. . . . . . . . . . . . . . . . . . . .87

4-29 $2.312 \mathrm{KHz}$ Linear Resonator Motion Measurement. . . . . . . . . . . . . . . . . .88

5-1 Optical Waveguide Layouts for Probing a Linear Resonator. . . . . . . . . . . . . . . . . . 97

5-2 Optical Path Traversed: a) Upper Diagram Demonstrates Input Optical Path and b) Lower Diagram

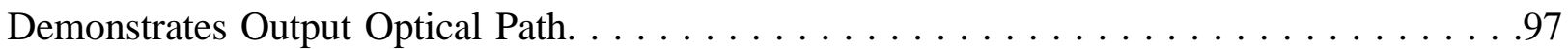

5-3 Cross Section of Inorganic Waveguide and Linear Comb Resonator. . . . . . . . . . . . . 105

5-4 Co-Integration of Inorganic Waveguide with MEMS Process Flow. . . . . . . . . . . . . . .107

5-5 Cross Section of Organic Waveguide with a Fully Released Linear Comb Resonator. . . . 113

5-6 Organic Waveguide Co-Integration with Prefabricated Linear Resonator Comb Device. . . 114

6-1 Cross Section of Inorganic Waveguide and Linear Comb Resonator. . . . . . . . . . . . . . 117

6-2 Cross-Section of Embedded Strip Waveguide. . . . . . . . . . . . . . . . . . 117 
6-3 Optical Profile of Inorganic Waveguide in y-Direction at a Wavelength of $1300 \mathrm{~nm}$. . . . 118

6-4 Optical Profile of Inorganic Waveguide in x-Direction at a wavelength of $1300 \mathrm{~nm}$. . . . 119

6-5 Optical Profile of Inorganic Waveguide in x-Direction at a Wavelength of $1300 \mathrm{~nm}$. . . 120

6-6 Optical Field Intensity Profile and Index Profile at Output. . . . . . . . . . . . . . . 121

6-7 Compilation of Optical Mode Profiles over a $1 \mathrm{~mm}$ Propagation Distance. . . . . . . . . . . 122

6-8 Compilation of Optical Mode Profiles over a 32 Fm Propagation Distance. . . . . . . . . . 122

6-9 Compilation of Optical Mode Profiles over a $0.1 \mathrm{~mm}$ Propagation Distance. . . . . . . . . 124

6-10 Compilation of Optical Mode Profiles over a 5 mm Propagation Distance. . . . . . . . . 125

6-11 Index of Refraction Profile for IOM simulation of $20 \mathrm{Fm}$ gap. . . . . . . . . 126

6-12 Optical Intensity Profiles for IOM simulation of $20 \mathrm{Fm}$ gap. . . . . . . . . . . . . . . 127

6-13 Simulated Normalize Output Optical Intensity of a Linear Resonator Displacement

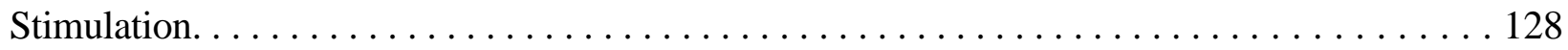

6-14 Endface Structure of Optical Waveguide. . . . . . . . . . . . . . . . . . . . . . . 129

6-15 Interference Pattern Created by Reflected Optical Signal. . . . . . . . . . . . . . . 132

6-16 Reflected Signal Interference Pattern. . . . . . . . . . . . . . . . . . . . .133

7-1 Cross Section of Organic Waveguide With a Fully Released Linear Comb Resonator. . . 134

7-2 a) left is the strip waveguide and b) right is the embedded strip waveguide. . . . . . 137

7-3 Single-Mode Profile at a Wavelength of $632.8 \mathrm{~nm} \ldots \ldots \ldots \ldots \ldots \ldots \ldots \ldots$

7-4 Single-Mode Profile at a Wavelength of $1300 \mathrm{~nm} . \ldots \ldots \ldots$. . . . . . . . . . . 140

7-5 Single-Mode Profile at a Wavelength of $632.8 \mathrm{~nm} \ldots \ldots \ldots \ldots \ldots \ldots \ldots \ldots \ldots \ldots$

7-6 Single-Mode Profile at a Wavelength of $1300 \mathrm{~nm} \ldots \ldots \ldots \ldots \ldots \ldots \ldots \ldots$ 
7-7 Single-Mode Profile at a Wavelength of 632.8 Fm.

7-8 Single-Mode Profile at Wavelength of $1300 \mathrm{~nm} \ldots \ldots \ldots \ldots \ldots \ldots \ldots \ldots$

7-9 Mask Layout of Polymer Waveguides. . . . . . . . . . . . . . . . . . . . 145

7-10 Multimode Waveguide Probe Structure Aligned with Linear Resonator. . . . . . . . . . 146

7-11 Optical Propagation of Partially Filled Modes in 100 Fm Polymer Waveguide. . . . . . . .147

7-12 Partially Filled Modes in Integrate Optical Metrology Structure. . . . . . . . . . . . . . . . . 149

7-13 Reflection of Waveguide Structure to Simulate Optical Gap between Linear Resonator and

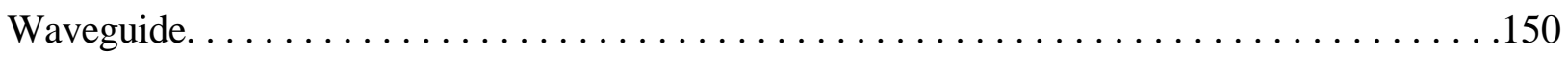

7-14 Region of Simulation of the Reflected Waveguide Structure. . . . . . . . . . . . . . 150

7-15 Optical Power Filling of Lowest Order Modes. . . . . . . . . . . . . . . . . . . . 151

7-16 Simulated Output Signal Intensity with Lowest Order Modes Filled. . . . . . . . . . . . 152

7-17 Optical Power Filling of Lower Order Modes. . . . . . . . . . . . . . . . . . . . 152

7-18 Simulated Output Signal Intensity with Lower Order Modes Filled. . . . . . . . . . . . 153

7-19 Optical Field Distribution of Relatively Higher Ordered Modes. . . . . . . . . . . . . . . 154

7-20 Simulated Optical Output Intensity with Relatively Higher Order Modes Filled. . . . . . .154

7-21 Optical Field Distribution in a 12 Fm Waveguide. . . . . . . . . . . . . . . . . 156

7-22 Simulated Optical Output using 12 Fm Guide. . . . . . . . . . . . . . . 156

8-1 Ultradel Process Flow. . . . . . . . . . . . . . . . . . . . . . 161

8-2 Final Ultradel Polymer. . . . . . . . . . . . . . . . . . . . . . . 162

8-3 25Fm Ultradel Polymer Waveguides. . . . . . . . . . . . . . . . . . . 168

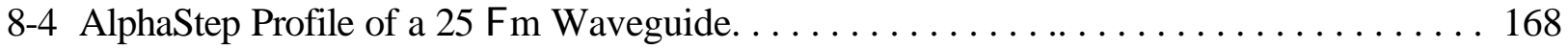

xiii 
8-5 MEMS Die with Close-Up of Linear Resonator Structure. . . . . . . . . . . . . . . . . . . . 169

8-6 SEM Photograph of Fully Released Linear Comb Resonator. . . . . . . . . . . . . . . . . . . . 170

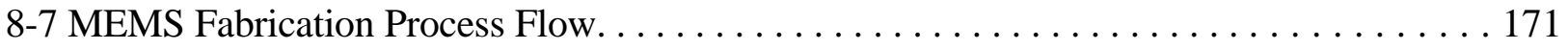

8-8 Oxide Removal Mask, Waveguide Mask and Cladding Mask. . . . . . . . . . . . . . . 173

8-9 Co-Integrations of Linear Resonator, Cladding and Waveguide Core. . . . . . . . . . . . . 174

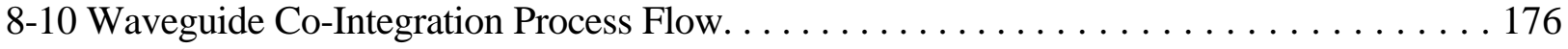

8-11 MEMS Die Mounted on Substrate Wafer. . . . . . . . . . . . . . . . . . . . . . . 177

8-12 Polymer Waveguide and Cladding Fabricate Next to Phase 2 Linear Comb Resonator. . 181

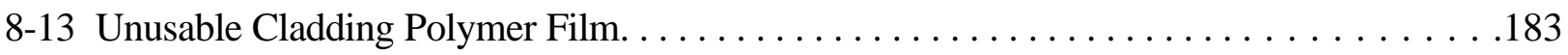




\section{Chapter 1}

\section{Introduction}

This research explores the feasibility of using integrated optical waveguides to measure the motion of microelectromechanical structures (MEMS). MEMS are small devices fabricated in silicon. They are free moving structures with dimensions on the order of several microns. This new silicon technology has many important applications as sensors and actuators, which is why it is being used to miniaturize large scale mechanical systems $[1,2]$. The motion of these devices can be submicron, making the devices hard to characterize. One way of detecting these motions is to use optical measurements. The work presented here explores how the optical measurement techniques can be integrated to create an in-situ measurement process that can characterize MEMS.

\subsection{Background}

Microelectromechanical systems are an exciting new class of silicon devices. Micron-scaled machines are being fabricated out of silicon, and the free moving devices are being actuated by electrostatic forces. Using processes adapted from micro fabrication, small simple machines (for example, membranes, cantilever beams, and hinges) and more complex structures (such as linear comb 
drives and motors) are fabricated on silicon wafers. The sensitivities of these devices to external forces, like pressure, temperature, heat, and light, make them excellent sensors. MEMS sensors are already being used as the standard triggers for air bags, and MEMS devices have been embedded in the tires of the space shuttle orbiter to monitor pressure and temperature [3,4].

The concept for MEMS devices started in the 1950's, and over the past four decades, processes have been perfected so that in the late 1990's, the technology is coming to marketable fruition. Just as the development of transistors allowed the integration of electronics, the development of MEMS is allowing the integration of mechanical devices. These mechanical devices are extremely sensitive to small changes in ambient forces and have been shown to act as good sensors, and they have an added advantage of being small and requiring low power. It is a technology that may not revolutionize the way we live, but one which advances the ability of existing technologies $[4,5,6,7]$. In the 1950's, MEMS were first fabricated using two forms of isotropic etching: alkaline solutions, which attack silicon crystal planes at different rates, and isotropic plasma etches. The etch processes, in combination with silicon oxides and nitrides to act as masks and etch stops, could be used to develop some basic MEMS structures, like diaphragms, trenches, and cantilever beams. This method of fabrication is called "bulk micromachining". Some MEMS devices are still being produced this way today because it allows structures to be fabricated on both sides of the silicon wafer. The reason this method of fabrication in some cases is not as desirable for fabrication is the etching process, particularly the wet etching, is not a standard process for VLSI fabrication. Some etches make the wafers fragile and create significant topology which also complicate bulk fabrication processes $[5,6,8]$. 
In the early 1980's, a new method of fabricating MEMS was developed at Berkeley. This new process is called "surface micromachining", because MEMS structures are built on top of the silicon wafer surface. Westinghouse had originally introduced the idea of creating devices by applying layers of structural films alternated with sacrificial layers in the 1960's. At Berkeley, this idea was developed further by using polysilicon films to fabricate structures and silicon dioxide as a sacrificial material. This advance in MEMS corresponded with the widespread usage of polysilicon and its maturation as a CMOS gate material. An example device fabricated using surface micromachining is shown in Figure 1-1. The device is a rotary side drive motor and was made using the Multi-User MEMS Processes (MUMPs) developed at MCNC. This foundry service was a DARPA and NSF supported program, developed so researchers had access to a cost effective MEMS fabrication. This foundry was recently bought by CRONOS. The MUMPS process is called a two layer process, because two layer polysilicon devices can be fabricated including an initial poly anchor $[3,4,5,6,8,9$, $10,12]$

While the processing is slightly different due to the thicknesses of the films, the surface machining process uses standard VLSI fabrication techniques. In the surface micromachining process the devices are built on top of a silicon substrate by layering and patterning films of polysilicon and sacrificial oxides. After the process is complete, the devices are submerged in a bath of Hydrofluoric Acid (HF) to etch away all the oxide layers. This process is called a release process because the 

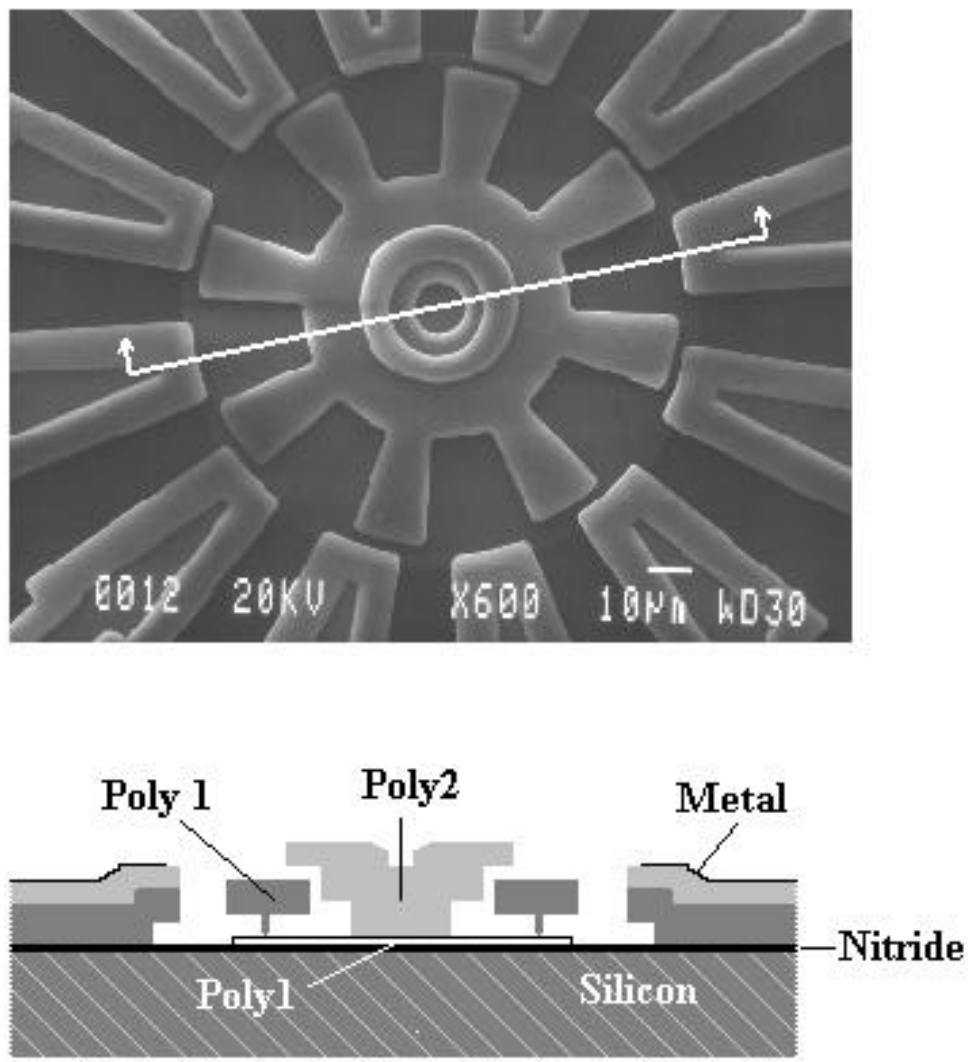

Figure 1-1: Cross Section of a Rotary, Side-Drive Motor (Not to scale.) $[12,13]$

patterned polysilicon layers now exist as free moving structures. The surface micromachining process outline of the MUMPs process at MCNC is shown in Figure 1-2. The limitations of the thicker films, which are on the order of microns, is that the topology becomes uneven after about four layers, limiting the patternability of any additional films $[9,10,12]$.

Despite its limitations, surface micromachining has an important advantage; the development of complex MEMS structures, such as linear comb drives and motors. The advantage of using already standard process is that MEMS devices can be mass produced, just like microchips. A second 


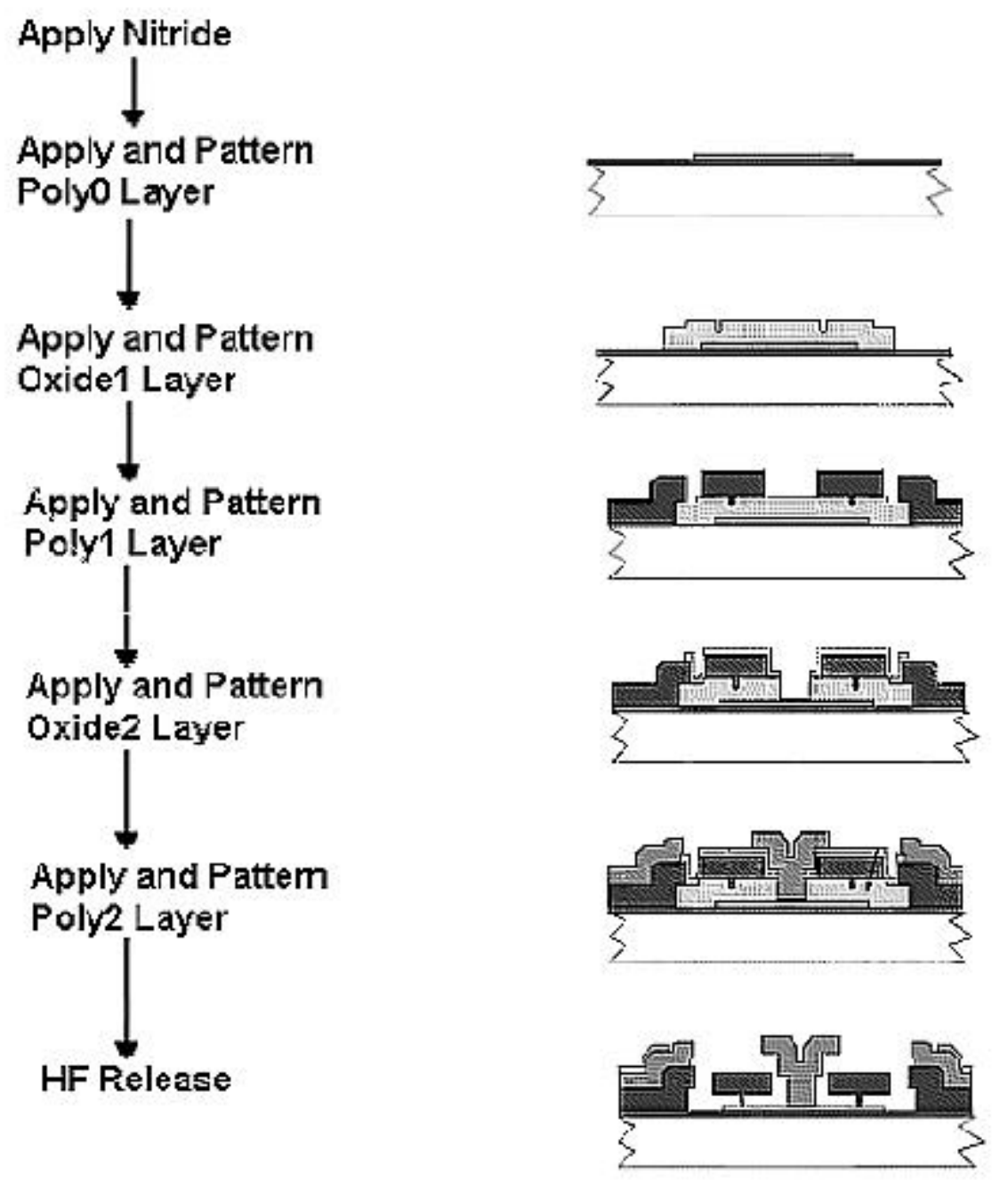

Figure 1-2: Process Outline, with Motor Cross-Section, for MCNC MUMPs Surface Micro-Machining Process [12].

advantage of surface micromachining is that it allows MEMS to be integrated with electronic circuitry on the same substrate. This is achieved by creating the electronic circuitry using tradition fabrication techniques and then masking the circuit areas while the MEMS devices are being fabricated. The cointegration of MEMS sensors and actuators with standard IC electronics provides an opportunity to connect real world forces with electronics and computers. This process method of masking areas to 
co-integrate different devices can also translate to other technologies, like photonic devices $[3,4,5,6$, $9,10,11,14,15]$.

\subsection{MEMS as an Emerging Sensor Technology}

The integration of MEMS with traditional electronics on the same substrate has great potential in the market place. Many possible applications have been proposed which apply across many fields. While the market for MEMS is currently in the automotive and medical fields, the marketplace for MEMS is expanding as the technology crosses over into the communications, aerospace and electronics industries. Just as the number of transistors that can be integrated on a chip increases the functionality of each chip, as the number of MEMS devices that can be fabricated on a chip increases the functionality of the MEMS chip increases. The chart in Figure 1-3 shows that as both the number of transistors and the number of MEMS devices on a chip increases, some very complex systems can be developed. The more advanced functions that may be achieved are Terabit/cm2 data storage, Digital Mirror Displays (DMDs) and distributed structural control (for example, active skins that can change the aerodynamic properties of an airplane wing). These complex functions require that the MEMS devices take on more sophisticated structures and control circuits $[1,4,7,10,16]$.

DARPA's research into MEMS is aimed at improving weapon systems and field intelligence. Their investment objective is to push MEMS technology to a higher degree of performance and to create MEMS out of new materials for specialized environments, for example silicon carbide can withstand higher temperatures and more rugged environments than silicon, so it would be necessary to use as a material for MEMS sensors which are mounted inside engines to monitor pressures and flows. 


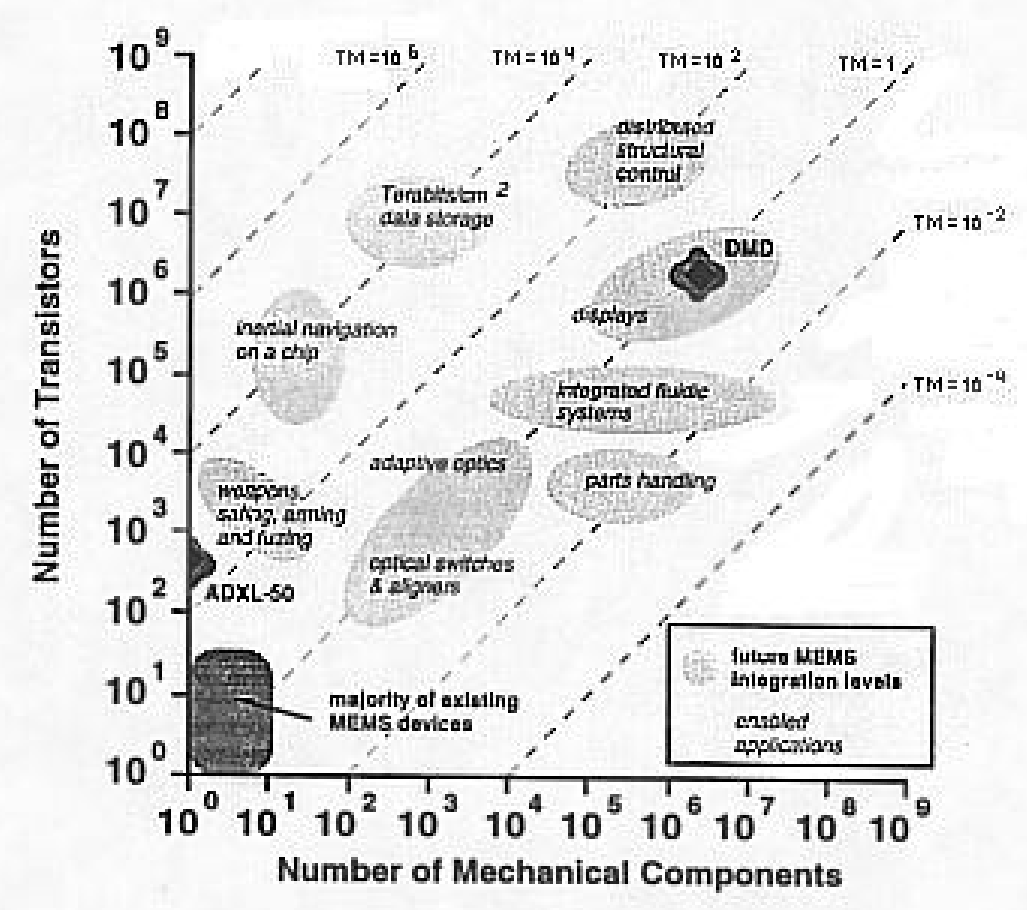

Figure 1-3: Predicted Development of MEMS Devices [8].

Defense applications for MEMS sensors and actuators are focused on three areas: inertial measurement, which will be used in weapons safing, arming and fusing systems, and navigation systems for vehicle and persons; distributed sensing and control, which will be used for situational awareness, miniature analytical instruments, biomedical sensors, friend-or-foe identification and condition based maintenance; and information technology, which is necessary for unattended sensing, mass data storage and low power, high resolution displays [8].

Today, the largest funding source for MEMS research is DARPA, but the driving applications of MEMS is being determined by the commercial market- a market which is demanding high performance, low cost sensors. Unlike DARPA's research into complex sensing networks, the commercial sector is fueling the applications for MEMS devices in some very mundane ways. Most 
commercial MEMS structures only contain one moving part, and some devices contain no moving parts at all. These motionless devices are classified as MEMS because they are made with the surface micromachining process developed for MEMS manufacturing [3, 8, 10].

The commercial sector is focusing on what MEMS can do now, which is act as high performance but inexpensively produced sensors. MEMS devices which act as sensors take physical signals, like pressure, temperature, or motion and convert that signal to an electrical measurement. There is a vast array of application for MEMS sensors, but the operation of these sensors can function in two basic ways. Besides measuring the direct motion of the device, MEMS sensors can also function by measuring the changes in resonance of their microstructure $[1,5,1,17]$.

The original sensor that started the development of the surface micromachining was a chemical sensor. A cantilever beam was fabricated with a thin film of a specific chemical absorbent film on its surface. The beam was driven at a resonant frequency and as a chemical gas was absorbed on the surface of the of the beam its mass was changed, subsequently the resonance of the beam was changed $[5,11]$

A very basic, and important MEMS sensor is an accelerometer. It is the standard sensor used for air bag deployment. One way an accelerometer can be made is to create a mass, called a proof mass, suspended on small thin suspension beams. When the sensor is accelerated the motion of the proof mass, created by the inertial force, can be measured. This measurement is usually performed by either, integrating piezoresistors on the suspension beams and observing changes in resistance, or by changes in capacitance between the proof mass and a sensing plate, which is fabricated next to the device. A different type of accelerometer that operates on a different principle is created by fabricating 
the proof mass with inter-digitated comb capacitive structures. A voltage is applied to the capacitive combs creating a restoring force holding the proof mass in place. When the inertial forces become strong enough to overcome the electrostatic force, a voltage is applied to restore the mass to it zero state. This method of acceleration sensing is more sensitive than the typical air bag sensors. Some capacitive sensors can detect sub-microgravity accelerations at 1 hertz bandwidth. These higher precision accelerometers are useful in navigational systems $[5,15,18,19]$.

A third type of accelerometer operates on an entirely different sensing principle. It uses a resonant structure. At Berkeley, a type of resonant accelerometer consists of the inter-digitated comb capacitor structure, but instead of applying a constant voltage, the structure is driven at frequency by a sinusoidal voltage. When an inertial force acts upon the proof mass, then there is a change in the frequency of the motion of the device, and this frequency shift from the drive signal is detected capacitively $[5,10,11]$.

\subsection{Future MEMS Applications}

Applications of MEMS sensors is looking beyond the low cost aspects and focusing on the uniqueness of the sensor devices. One important, future development is applying MEMS devices in distributed sensor networks. MEMS sensors can be distributed throughout several systems in automobiles and airplanes, for example, and the continuous monitoring of specific conditions can create a "repair as needed" maintenance routine. Another field of growth is data storage. The amount of data that can be stored on any material is determined not only by the storage medium unit cell, but also by the size of the read/write head of the device. It has been proposed that atomic data storage can be 
created by moving atoms with an atomic force microscope head, but only MEMS devices will be small enough and fast enough to move the head efficiently $[2,20]$

The drive to fabricate new types of MEMS sensors is threefold. First, the MEMS devices are unique in size. Microscopic sensors can perform the same functions as larger scale systems. This will allow bulky sensor systems to be reduced to portable or hand held systems. Minute devices have additional applications, because they can be placed in situations where bulky sensors will not fit. Second, being fabricated out of silicon the sensors can be fabricated in mass like IC chips, and they could be just as cheap as IC chips because the development and manufacturing costs are distributed over millions of produced components. While it may not be intuitive that silicon would be a good material to make moving structures, it has several mechanical properties that make it a good choice for fabricating MEMS. Silicon has a hardness of $850 \mathrm{~kg} / \mathrm{mm}_{2}$, which is almost equivalent to quartz and a tensile strength of $6.9 \times 10_{10} \mathrm{dyne} / \mathrm{cm}_{2}$, which is equal to that of steel. The one limiting property is its brittleness. The third advantage of integrated sensors is that the fabrication process of MEMS structures allows for the manufacturing of complete mechanical and electrical systems. Micromachining is a process that utilizes the same deposition and patterning techniques as VLSI fabrication; the only significant difference is the thicknesses of the films. Electronics can be co-integrated by fabricating them first, and then masking these areas during the MEMS fabrication. Large systems with hundreds of individual moving parts and the accompanying control circuitry can be fabricated side by side with little or no assembly. The batch processing of the MEMS devices is what will enable very complex systems to be fabricated on a single die, and the process facilitates hundreds of devices to be fabricated in a small area, which will allow for very compact sensing arrays [2, 6, 11, 14]. 
Advancement of MEMS technology is showing that devices can be used for much more than just sensors, which monitor the environment, but that they can be used as actuators, which affect the world around them. There are also MEMS devices that can also be driven by an electrical signal to create motion and apply force. The co-integration with microelectronics will allow MEMS devices to sense the world around them, make logical decisions, and exert forces to actuate change. Some example devices, like deformable mirrors, have been used to steer laser beams, and miniature motors have shown that they can pump fluids. A single MEMS actuator may only exert a very small force; however, the ability to fabricate hundreds of devices in a small area means that collectively MEMS devices can exert large forces $[6,7,21]$.

\subsection{Monitoring as a MEMS Enabling Technology}

The detection of motion of all MEMS devices is critical for characterizing their behavior. Physical characterization of MEMS motion is not as well understood as the electrical characteristics. The relative scale of forces that effect MEMS sensors and actuators are not the same as those experienced in regular-scaled systems. In the micron world, gravity is negligible and other, weaker forces become very important, like static electricity, air resistance and surface tension. Some forces like friction may be the same, but new forces like 'stiction' must be understood. While the surface micromachining process has been developed as a batch fabrication method for MEMS, it is also one of the main factors limiting the production of MEMS actuators. Multiplicity of devices has a lot of potential, but currently the percent yield of functional devices can be low. The yield rate is dependent upon many factors, but they center around the sticking of the devices during the 
release process. The rinse process after a release bath introduces water in-between the newly released MEMS structures and the substrate. Because these gaps are on the order of a micron the surface tension of the water can "glue" the free moving structures to the substrate; this phenomenon is called "stiction". As the water evaporates, residual dissolved silica in the rinse can redeposit creating solid structures between devices, this is called "release-related stiction". The geometry of the design and the type of materials used affect how much sticking can occur. Other issues also come into play with the use of more complex structures and actuators. After release, the free moving structures are very delicate and back-end processes, like sawing and bonding can create more sticking problems. There are also issues with packaging, like how to seal the devices and the electronics from the environment, while allowing the sensors and actuators to act on the surrounding atmosphere. Another important limiting factor to the current production of actuators is that of the friction and wear. Because MEMS can exert forces on objects and fluids, the interactions of forces and wear need to still be studied. While research is being furthered in the area of micro actuators, it will be another decade before they reach marketable fruition. Research over the past two decades has focused on refining the fabrication process and developing new types of devices, but a new direction of study is necessary. An understanding of these forces and their effects on the motion of devices will be important for furthering the technology $[3,7,8,22,23]$.

When characterizing MEMS behavior, there are two types of devices discussed: rigid and flexible devices. An example of a rigid device is a micromotor. A picture of this device can be seen in Figure 4-1a. This is a solid device that turns on bushings and the rotational stage does not change shape. The characterization of rigid device behavior is called microtribology. If actuators are to be 
used to pump fluids and exert forces on outside systems, the wear and tear on these devices must be studied to predict performance reliability. Therefore it is important to develop measurement techniques that will perform over the lifetime of the devices while in operation. An ability to monitor the use and the performance of MEMS devices also adds a method for optimizing device operation by providing a feedback signal [3].

Motion of deformable MEMS devices consists of displacements, torsions and rotations. An example of a deformable device is a comb resonator as seen in Figure 1-4b. This device consists of a stage suspended on two deformable spring structures, and it oscillates in a plane parallel to the wafer surface. These motions can be linear or nonlinear, depending on the actuation signal intensity, the geometry, and the degree of motion. While computational models can be made to predict each type of motion, they are not always accurate (especially near resonance) and device characterization must be done on actual devices. The motion of these devices needs to be measured on a submicron scale making standard measurement techniques difficult. Some motions can be seen under optical microscopes and CCD cameras, but only bulk optical laser metrology and scanning electron microscopes can be used to detect fine motion. This is not feasible for 'in situ' measurements or lifetime monitoring. An added difficulty is that the devices do not always perform the same under vacuum as 


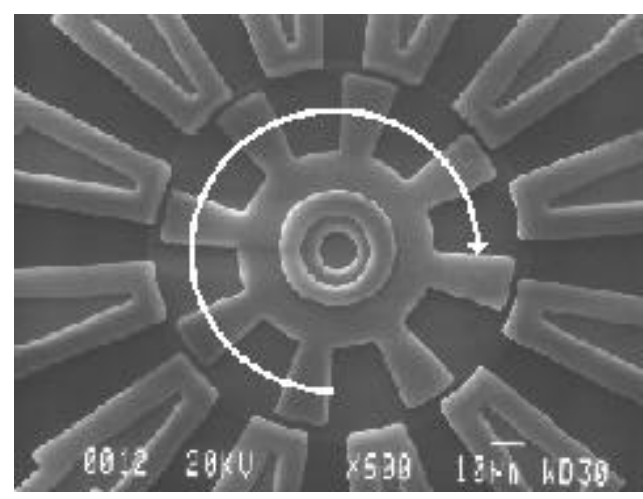

(a) Rotary side drive motor developed by MCNC. [12]

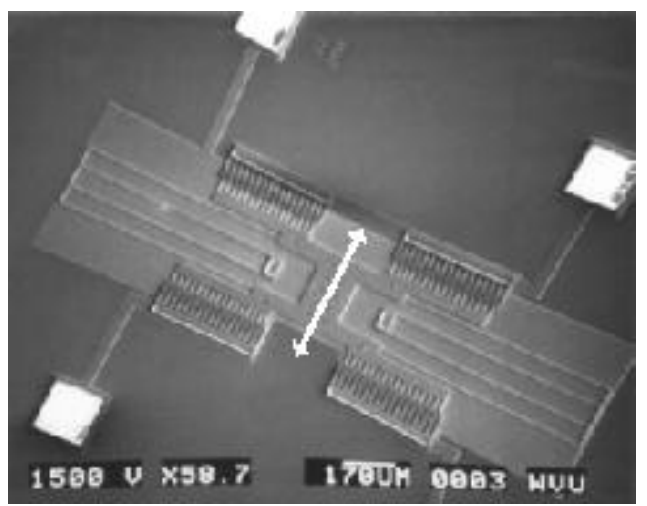

(b) Linear resonator stage designed at WVU. (Arrows indicate the direction of motion.)

Figure 1-4: Example MEMS Devices

they would at atmosphere or in any other environment. MEMS devices that are submerged in fluids will experience fluid resistive forces that they would not experience in a vacuum $[24,25,26]$.

One type of measurement that can be used to detect motion of devices is capacitive measurements. While studies have been made using capacitive signals to detect displacement, there are several limitations to using electrical measurements. Detecting the small impedance changes at 
resonance is extremely difficult. The voltage signals driving the stage are large, in the tens of volts range. This creates a coupling overlap between the input signal and the measurement signal. The measurement signal is further corrupted by parasitic capacitances with the grounding planes of poly0 and the sample substrate. All of these noise sources must be accounted for up to the resonant frequency. Corruption of the signal creates more problems during amplification, which is required to measure the low level of feedback signal. Larger capacitor areas can be used to increase electrical feedback signals, but this averages physical motions over larger areas. Capacitance size can also be limited by the device structure size. Determining the fine modal changes of mechanical motion is very important when studying the failures caused by defects [15, 27, 28].

New methods of measuring motion in-situ must be developed. What is desired is a method of measuring very fine displacements of these MEMS structures. The measurement must be focused on very small parts of the device to determine mechanical deformations over a wide range of mechanical frequencies. The measurement method should be isolated, and the measured signal should be decoupled from the mechanical system. It would also be beneficial if the measurement technique could be integrated and packaged on chip like most electrical test circuitry. Repeatability of measurements over the lifetime of device operation is also desirable for monitoring. MEMS devices are being used as sensors in critical systems, and the failure of a MEMS device is important knowledge in creating fault tolerant systems. 


\subsection{Optical Metrology}

One potential method of in situ measurement is optical measurement. While the motion of the devices can be small, they are still large compared to coherent optical wavelengths, and the small, micron scaled features are large relative to possible optical apertures. Optical measurement is non-invasive and can be used to measure both static and dynamic displacements. Because the optical signal is not electrical, it cannot be capacitively coupled to the input voltages [24].

Research has been done in the area of using macroscopic, bulk optical probes to study the motions of MEMS devices. However these techniques can only be used to test and characterize in a lab setting, which is important for developing the technology, but they can not be readily used to monitor the MEMS devices over their entire lifetimes while still retaining the small size and light weight advantages of MEMS. The bulk optical probes focus lasers onto the MEMS devices and take measurements by either studying the deflections of the beams or using laser vibrometer measurements. Because these systems use lasers, bulk optics, and bulk optomechanics, this method of measurement is called bulk optical metrology (BOM). While the measurements do use optical signals, which are the types of measurements that are desired, what is needed is the ability to perform these measurements insitu, which gives the advantage of an isolated signal that is non-invasive. The large-scale lasers and optical components of BOM mean that the devices cannot be studied in actual operational environments. An integrated measurement technique is needed for lifetime monitoring and control [24, $29,30]$. 


\section{Chapter 2}

\section{Research Motivation and Outline}

\subsection{Current Micromachined Optic Devices}

Over the past two decades, while the MEMS fabrication methods and technology were being developed, the technology of micro-optics, a set of miniaturized optical components and devices, has also matured. The merging of these two technologies has created a class of devices called microoptoelectromechanical systems (MOEMS). Micro-optical devices are integrated forms of lenses, diffractive elements and refractive elements. Since the fabrication of some integrated optical devices utilize processes which are similar to surface micromachining, it is logical that the two technologies can be combined together. Coupling optical devices with small actuators for positioning and movement has created integrated versions of bulk optical systems. The application of MOEMS technology has expanded the functionality of micro-optic devices by creating the ability to move the micro-optic devices with electrical signals $[8,25,31,32]$.

Like optical interconnects, MOEMS are usually classified into two general categories, in-plane and out-of plane, depending on the direction of the light propagation in relation to the MEMS device. Development of in-plane micro-optic devices has focused upon using optical waveguides interconnects. 
However, most research into planar MOEMS has focused largely on micro-optical benches. While these structures are planar, they are not really aligned with structures along the surface of the wafer, and therefore are not useful for Integrated Optical Metrology ( IOM). The type of planar micro-optic integration that has received little to no attention is the use of planar optical waveguides. Out-of-plane MOEMS applications have been explored more extensively. These applications operate using one of many possible optical interactions. Some application use a moving or positionable device to interrupt an optical beam. Another type of MOEMS uses a deformable membrane which creates adjustable microcavities above vertical emitting laser diodes $[8,21,32,33]$.

A well known MOEMS device is the Texas Instruments reflective mirror, projection display, which utilizes optical redirection. Micron scaled mirrors are electrostatically tilted, redirecting light shined upon the mirror surface. This tilting directs light toward or away from the display screen, and turns pixels on and off $[4,32]$.

There are several optical integration methods that can be used to monitor the motion of MEMS devices. Jeremy Dawson, who is a Ph.D. student in Electrical Engineering, has completed an initial exploration of free space optics by using through-wafer signals to monitor the position of a MEMS linear resonator. This research will focus on the use of planar integrated optics. Optical waveguide co-integration with surface micromachined structures has the potential to enable integrated optical tests for metrology and state feedback functions for monitoring of complex MEM systems [33]. 


\subsection{Integrating Optical Monitoring}

Planar optical monitoring can potentially be achieved by integrating optical waveguides in the plane of the devices. Careful planning can allow the waveguides to be fabricated next to the devices, so that an optical signal can be reflected off the edge of the MEMS structures. This co-integration of waveguides to use as optical probes guarantees a permanent positioning and alignment of the guide to the device on the chip, which is necessary for in-situ measurements. It also allows for on-chip monitoring of the device over its lifetime of operation and adds the capability for closed loop feedback control.

IOM will be important for device characterization, microtribology, and signal feedback control. It is a non-invasive, non-destructive testing method. An integrated optical signal being reflected or redirected by a microstructure can be a function of MEMS motion and therefore be used as a monitoring signal or as feedback for the control circuitry of the MEMS devices. Integrated optical metrology can rely on the well developed technologies of micro-optics and optical interconnections [24].

Adding integrated optical waveguides is adding another layer of technology and creating a mixed technology by placing optical elements alongside microelectronics and MEMS devices. The evolution of new devices utilizing these different technologies will develop in a similar fashion to the development of complex microelectronic circuits. Increased functionality of ICs was achieved by assembling groups of simpler functions, which were standard, generic cells. By drawing upon previous designs of simpler functions, higher order functions were created. This same design technique can be 
applied to the MEMS technology and the integrated waveguide technology, as discussed in the next section.

Implementation of IOM will require the co-integration of optical waveguides with MEMS structures. Insertion of the waveguide fabrication process into the MEMS fabrication process requires that both processes be examined for compatibility. Issues such as thermal cycling, material adhesion, material resistance to fabrication and environmental processes, and topology are just some of the possible considerations. An important, defining factor which will determine the entire order of the fabrication procedure is the choice of material for the optical waveguides. The optical material must be resistive to Hydroflouric acid (HF) used in the MEMS release process if both integration processes are to be achieved on the same substrate.

\subsection{Development of Integrated Optical Monitoring Cells}

Standard mechanical functions are being designed as basic MEMS devices, which produce specific motions. Basic MEMS motions can be divided into three basic categories: translational, rotational, and torsional. Translational motion is exhibited in devices, which produce linear displacement along an axis. An example device, which produces this type of motion, is a linear resonator comb drive. Rotational motion is exhibited in devices, which rotate around a central axis. An example of this type of motion is demonstrated in micro-motors. Torsional motion is exhibited in devices, which twist around an axis. Standard MEMS cells are specific devices which produce one or a combination of these motions that can actuate other MEMS devices [15]. 
As optical waveguides are integrated with these standard MEMS cells, a basic set of IOM cells will be developed. Each basic MEMS device will require specific waveguide configurations to measure the motion of its devices. The development of IOM cells will create a third set of standard cells to add to the technology. However, the co-integration of optical waveguides with MEMS structures has not previously been considered. Understanding the development of this technology faces certain challenges. This research will analytically explore the optical signal intensity to evaluate its effectiveness for measuring MEMS displacement, and will evaluate the feasibility of a co-integration process for fabricating optical waveguides with surface micro-machined MEMS devices.

\subsection{Research Statement}

This research will explore the feasibility of using integrated optical waveguides to measure the motion of MEMS structures. This dissertation will study the co-integration of optical waveguide probes with one specific standard MEMS cell, the linear resonator comb drive. Four factors were used in this decision. First, the linear resonator device is deformable and the measurement interest is focused upon the actual device motion, not upon the changes in motion due to device degradation. Secondly, the device moves translationally along a single axis, when operating in a linear range. Simplifying the motion measurement to an axial displacement. Thirdly, the linear resonator is a basic device that is utilized in many applications $[7,18,19,28,34]$. Operating without mechanical loads, they act as gyroscopes and accelerometers. Comb drive resonators can also drive mechanical loads, to operate other MEMS

devices like motors and grippers. Fourthly, the device is a simple design, which can be fabricated using 
the MUMPS foundry service at MCNC. The fact that the structure can be made utilizing only the poly0 and poly1 layer minimizes the problems that can occur with the release process.

This research will focus on the two main aspects of understanding the use of optical waveguides for integrated optical monitoring: the analysis of the measurement signals and the optical waveguides that carry them, and the fabrication issues of co-integrating an optical waveguide fabrication process with the surface micro-machining process. The following is a breakdown of this research.

\section{Study of the optical waveguides and signal intensity.}

- Design of a linear comb resonator for IOM studies.

- Develop an understanding of linear comb resonator motion.

- Determining the optical signal intensity changes with device motion for single mode and multimode guides using a fiber optic metrology method.

\section{Optical waveguide co-integration.}

- $\quad$ Evaluation of optical waveguide materials for co-integration.

- Determination of a process flow for single-mode, inorganic waveguide co-integration.

- $\quad$ Simulation of the optical behavior of a Silicon Oxide single-mode waveguide probe.

- $\quad$ Simulation of the optical behavior of a multimode Polymer waveguide probe.

- Development of polymer waveguide fabrication at WVU.

- $\quad$ Co-integration of polymer waveguides with MEMS devices. 


\subsubsection{Study of the optical waveguides and signal intensity}

The basic principle of IOM will be to measure motion or displacement of a MEMS device by probing it with an optical signal. This research proposes the use of integrated optical waveguides to both introduce the optical signal to the MEMS device and to collect the reflected signal. A primary way of detecting either device motion or displacement is to reflect the beam off of the device and study the signal intensity change of the reflected optical signal to determine changes in optical path length due to device displacement. The change in optical signal in this scenario can be created by changes in optical losses due to variations in optical path length, or by changes due to interference effects if the optical source is coherent. Due to the possible importance of the source coherency, it will be necessary to examine optical waveguide probing structures for both single mode and multimode waveguides.

Besides using waveguides to introduce the optical signal to the MEMS device, integrated waveguides will be used to collect the reflected optical signal and direct it towards an optical detector. Because the waveguides will be carrying the output signal, it will be an important part of this research to study the layout of the optical waveguide structures to minimize optical losses in the guides, which will degrade the optical measurement signal.

Before studying the optical signal and its effectiveness for measuring device motion, an understanding of the linear resonator motion will be developed. The linear resonator is a test structure, and understanding its operation and predicted motion will be critical in understanding how to measure this motion. Because the focus of the research is on the ability of the optical signal to measure device motion, it will be assumed that the linear resonator is operating in a linear region. Being a deformable 
device the resonator can display both linear and non-linear behavior depending on its operational voltages and frequencies.

Initial optical measurements of linear resonator motion will be evaluated using a fiber optic probe system. While not integrated waveguides, the fiber optic system will serve to indicate the measurement techniques that can be performed using optical waveguides. This experiment will also indicate the fundamental difficulties with optical measurements, like alignment and stability, that can be solved utilizing co-integrated waveguides. The development of an optical waveguide layout for optical probing of the linear resonator will be dependent on several factors such as waveguide size and optical material. Analysis of these structures will require mathematical modeling and simulation of the optical wave front as it propagates through the waveguide structure to explore the constructive and destructive interference experienced as the resonator stage changes placement during operation which determined the intensity of the output signal.

\subsubsection{Optical waveguide co-integration}

To demonstrate the co-integration of guides with MEMS devices, the fabrication process for polymer waveguide probes with prefabricated MEMS structures will be developed. Research work targets the application of multimode waveguide structures to achieve integrated optical metrology functions for MEMS. Specially designed 'split-comb' linear resonator devices which enabled coupling of the waveguide to the resonator stage for position feedback measurements for the co-integration experiments were designed at WVU and fabricated at MCNC. 
Initial fabrication studies will focus on the development of a process for polymer optical waveguide fabrication at WVU. After Ultradel Polymer integrated optical waveguides are fabricated, a follow-on fabrication study will be performed to examine the co-integration of the waveguide process with pre-fabricated MEMS devices.

\subsection{Outline of Dissertation}

This research is to explore the use of optical waveguides for integrated optical monitoring of MEMS devices. The remaining part of this dissertation will evaluate the feasibility of waveguide configurations for measuring the device motion with an optical signal by analyzing the optical signal and simulating losses in the waveguide structure. It will also present the implementation of co-integrating optical waveguides with MEMS devices, by studying the possible materials that could be used for fabricating the guides, and by developing a fabrication process for integrating waveguides with surface micro-machined devices. The research of these important issues will create a basis for further research into IOM and MEMS devices.

Chapter 3 will present the operation of linear resonator MEMS devices. An equation which represents the displacement of the device will be derived mathematically. The equation shows that the displacement of the linear resonator, $\mathrm{x}(\mathrm{t})$, is linear with driving voltage, $\mathrm{V}_{\mathrm{s}} \sin (\mathrm{t})$ if driven with opposing stator combs. The equation remains linear if the spring constant, $\mathrm{k}$, is assumed to be linear. All the other variables in the equation are either constants that are determined by the design of the structure or the bias voltage, $\mathrm{Vb}$ (which is a DC voltage) or forces created by the displacement of the device. This 
mathematical model is an idealized model of the device motion, but it provides a basis for understanding the types of displacement that will be measured by the optical monitoring signal. [25, 26, 27]

Chapter 4 presents the use of a fiber optic probe to study the motion of a linear resonator MEMS device. An optical fiber will be used to introduce an optical signal on the edge of a stage of a linear resonator MEMS structure. The resonator stage will be moved by either electrostatic actuation, or by manual probe manipulation. As the optical signal is reflected off the edge of the stage, the change in light intensity will be measured.

The fiber optic probe is actually a form of Bulk Optical Metrology (BOM), not a form of IOM. By performing an BOM experiment, a basis of comparison and expectation for IOM will be developed, and the experiment will show some of the principles that will need to be addressed when using guided wave optics. The chapter will present the experimental setup, the measurements, and a discussion of results.

Chapter 5 will present background information that is required for developing an IOM system for MEMS devices. While discussion will present the development of optical metrology cells to create a design set which will accompany the development of complex MEMS structure, the focus of this research will be on probing an linear resonator stage. A section will focus on the layout of integrated optical waveguides with MEMS, and possible modifications that may be required. More detail will be presented on the structures to show the theoretical operation of the optical measurement and yield a predicted output signal of the probe waveguide using optical modeling simulations.

Also included in this chapter is a discussion of the physical realization of the optical waveguide structure. A portion of this chapter will present the different type of materials that can realistically be 
used to fabricate integrated optical waveguides with MEMS. Two major classes of materials are silicon dioxide waveguides, and organic or polymer waveguides. Each material has its own set of advantages and disadvantages that will be discussed. This chapter will present proposed process flows for realization of both of these major classes of optical materials.

Chapter 6 will present a methodology for using silicon dioxide as a material for fabricating the integrated optical waveguide probes. The chapter will present computational models for the silicon dioxide waveguides for the process that was developed in the previous chapter. Accompanying analysis will explore the quality of signal that can be expected from the waveguide setup and appropriateness of measurement of this proposed IOM. The critical part of this research in proving the feasibility of guided wave IOM is the ability to use the optical signal as feedback for device displacement or motion. By studying single mode waveguide implementation, the effectiveness of signal interference due to changes in optical path length due to device motion will be evaluated.

Chapter 7 will present the methodology for using polymer, integrated waveguides to measure MEMS device motion. As with the silicon dioxide material, polymers have certain advantages and disadvantages. The properties of the material and how they affect the co-integration of the waveguides will be discussed. A significant section of this chapter is devoted to the simulation modeling of these types of waveguides. Predicted signal integrity and appropriateness of measurements will also be presented.

Chapter 8 of the dissertation presents the experimental development of a co-integration process for polymer waveguides with prefabricated MEMS structures and the difficulties which were identified. This chapter will start with an overview of the MEMS fabrication process and the method of insertion 
of the waveguide fabrication process. The next section will present the selection of optical polymer material and the successful development of the fabrication process for integrated waveguides uising the Ultradel 2000 series optical polymer produced by AMOCO. The next section of the chapter will discuss the development of the co-integration process and the difficulties encountered. A discussion of the encountered barriers in this development and potential, future directions for to the process development.

Chapter 9 will include a presentation of a summary of the research findings and conclusions, and a discussion of future work. 


\section{Chapter 3}

\section{Theoretical Calculations}

\subsection{Microelectromechanical Devices}

There are numerous designs of MEMS structures. However, all MEMS actuators can be classified into two general categories: deformable and solid mechanisms. Deformable micro-actuators are devices that change their shape, at least in part, as they move, such as membranes and deformable beams. An example of a deformable MEMS device is the linear resonator which is shown in Figure 31a. Solid mechanisms are micro-devices that are more rigid and therefore require bearings and joints; an example of these devices is a micro-motor, which is shown in Figure 3-1b. Each type of actuator has its trade-offs, deformable devices have limitations in motion and distances traveled, but mechanisms have difficulty with friction [15].

The focus on the measurements of performance of the two different classifications of devices, takes two different directions. Measurement of a deformable device tends to focus on the measurements of the device behavior during operation. Interest centers on finding over which range of operation the device is acting linear. Other operation parameters, such as resonant frequency, 


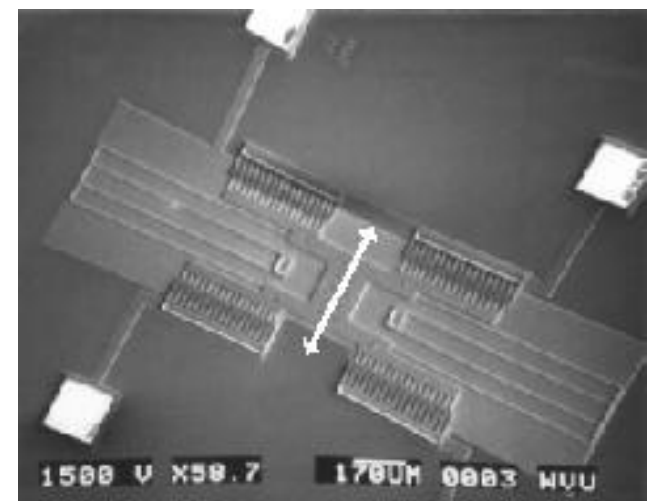

a.) Scanning Electron Microscope Picture of a Linear Resonator.

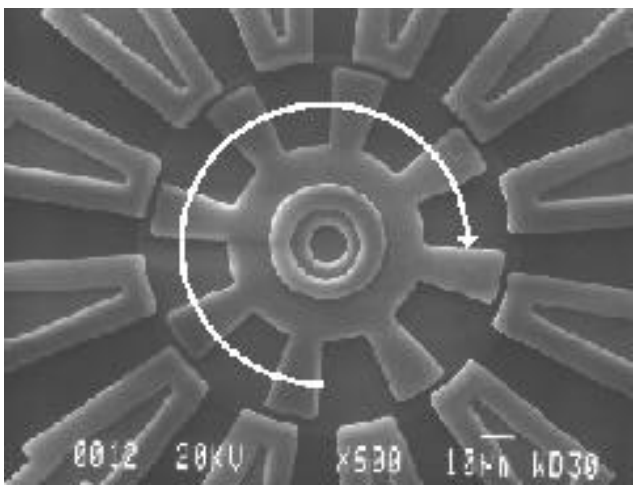

b.) Scanning Electron Microscope Picture of Micro-motor. [13]

Figure 3-1: Example Microelectromechanical Devices.

maximum deflection, and phase shift are also normally measured. Because rigid mechanisms experience different physical forces than the deformable structures, especially friction, the measured device behavior of interest tends to be performance over time. These particular measurements, known as microtribology, are used to calculate characteristics like failure rate and are used to understand the effects wear have on the performance of the device [15, 18, 23, 25].

\subsection{Linear Comb Resonator}

The goal of this research is to explore the feasibility of the fabrication of integrated optical waveguides with a MEMS for the purpose of measuring and monitoring the MEMS device motion. To explore waveguide based integrated optical metrology (IOM), an appropriate MEMS device had to be chosen for experimentation. Characteristics that were looked for were, one, a device which was generic and in wide use as a generic "standard cell" building block in MEMS, two, a device with a 
relatively straightforward layout that could facilitate easy alignment and fabrication of a waveguide, and three, a device which demonstrates simple motion that can be measured directly. The device chosen was the linear comb resonator, which has a simple design, and can be fabricated using the MUMPS foundry service at MCNC.

A picture of one of the WVU designed structure is shown in Figure 3-2 and a SEM image of released MUMPS fabricated device is shown in Figure 3-1a. The fact that the structure can be made utilizing only the poly0 and poly1 layer minimizes the problems that can occur with the release process. One modification added to the device pictured here, is a gap, which was designed in the center of the combs on the stage and in the ground plane under the stage. This gap divided the combs into two groups on each side. The purpose of the gap in the structure was to create a space for introduction of the waveguide, and to create a region along the resonator stage edge where optical measurements can be taken.

A second reason for choosing the linear resonator device is that it is deformable and the measurement undertaken can focus on the actual device motion, not upon the changes in motion due to device degradation. The device moves translationally along a single axis, when operating in a linear range. Simplifying the motion measurement to an axial, one dimensional displacement. Linear device operation is determined by the flexure structures and the intensity of the driving signal. As long as the amplitude of the voltage drive signal is not set too high, the resonator will operate within a linear region $[18,19,28,34]$.

Linear comb actuators are a very significant MEMS structure. The linear resonator is a basic device that is utilized in many applications. Without mechanical loads, these structures have been used 


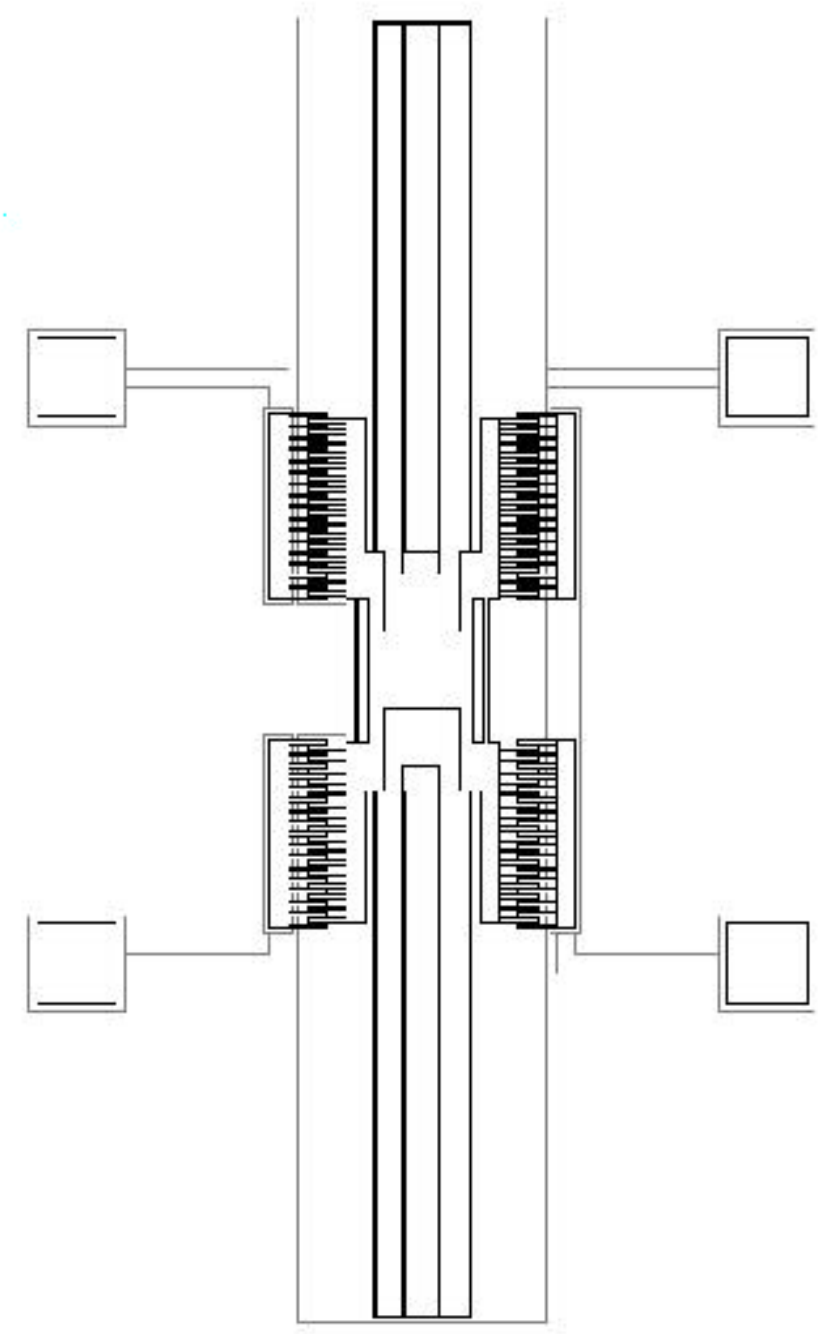

Figure 3-2: Linear Resonator Device

in force-balanced accelerometers, resonant accelerometers, and resonant gyroscopes. Comb drive resonators can also become actuators which drive mechanical loads to operate other MEMS devices micro-grippers and micro-engines [7, 18, 19, 30, 34]. 

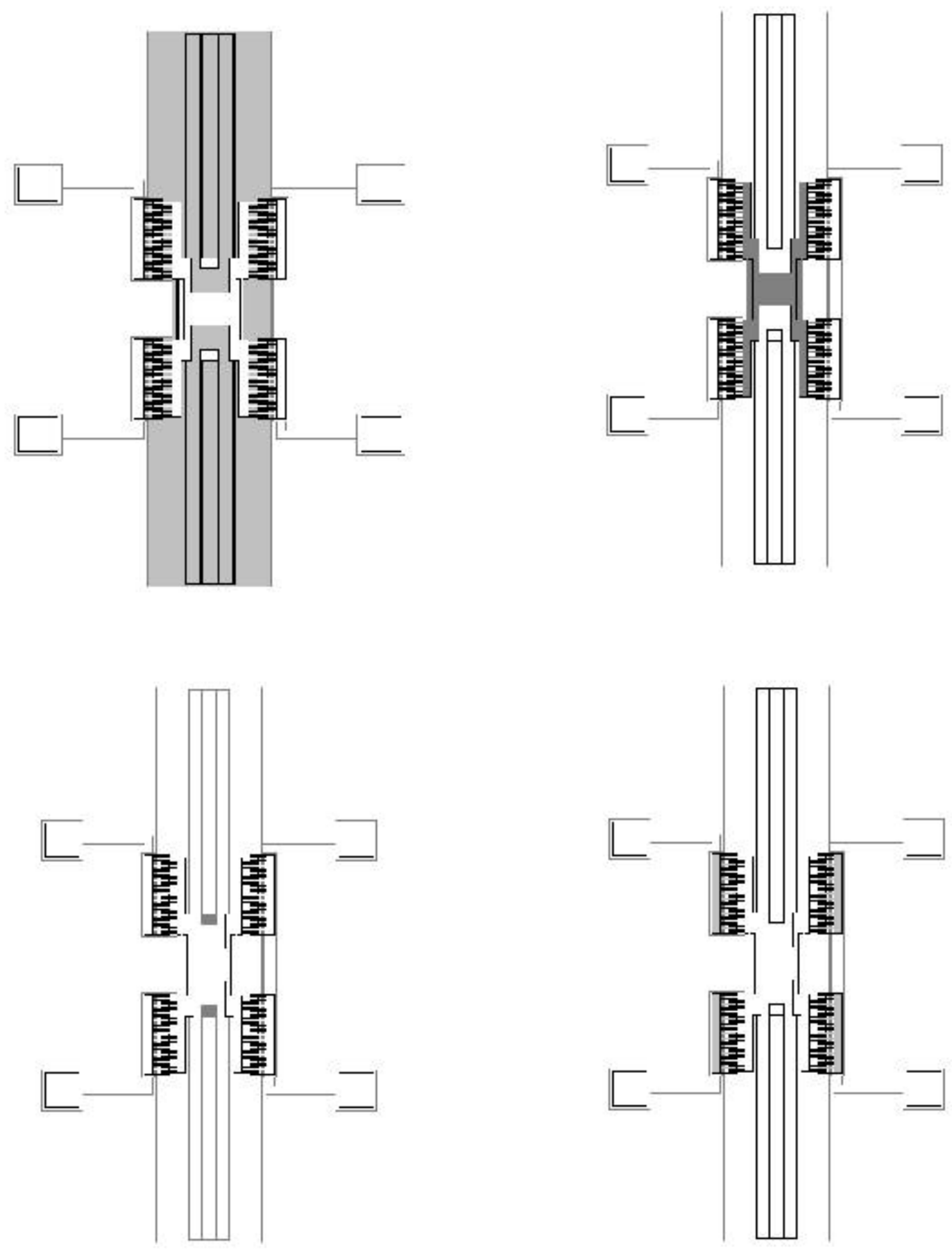

Figure 3-3: MEMS Figure Layout Emphasizing a.) Ground Plane, b.) Resonator Stage, c.) Folded Flexures, and d.) Stator Combs 


\subsection{Linear Comb Resonator Operation}

As seen in Figure 3-3 the linear comb resonator consists of four components. The first component is a ground plane, which is highlighted in Figure 3-3a. This layer under the resonator structure is often referred as a ground plane, though typically it is driven at the same voltage the resonator is. Its purpose is to shield the resonator from capacitively coupling to the silicon substrate. The second structure component is the resonator stage, which is highlighted in Figure3-3b. This is a large structure which is free floating above the ground plane. Being the largest part of the structure it contains most of the mass of the device, and in non-loaded applications it acts as a proof mass. The resonator stage is supported by two folded flexures, shown in Figure 3-3c. Each folded flexure consists of four narrow beams which are connected together. The two inner beams are anchored to the substrate at one end, this anchor also electrically connects the resonator stage to the ground plane, and the two outer beams connect to the stage itself, supporting it above the substrate. Highlighted in Figure 3-3d, is the stator comb drives. These devices are the stationary comb structures on the right and the left. The inter-digitated fingers of the stator comb drive and the resonator stage creates a capacitive coupling which drives the stage by changing the electrostatic voltages.

Electrostatic actuation of the inter-digitated comb fingers can provide displacement or sinusoidal motion in a direction parallel to the surface of the substrate. The comb devices are used in tandem for actuation because together they provide a linear force over the motion of the structure. Operation of this type of actuator was first demonstrated by Tang and Howe [34].

The typical method of operation is to continuously drive the linear resonator with a sinusoidal signal, especially if the device is being used as a resonance style detector. For the linear comb 
resonator to be drive the stationary comb fingers must be set at a bias voltage. Either side being held at an equal but opposite voltage, $+\mathrm{V}_{\mathrm{b}}$ and $-\mathrm{V}_{\mathrm{b}}$. Then the stage, which is electrically connected to the ground plane through the flexures, is driven with a sinusoidal signal which has a peak voltage of $\mathrm{V}_{\mathrm{s}}$. These voltage signals are shown in Figure 3-4. When the drive signal is in the positive half of the sinusoidal cycle, the voltage on the stage is increasing positively. The voltage potential between the stage and the right comb structure is increasing, creating a coupling force between the two.

Simultaneously the voltage potential between the stage and the left comb is decreasing and the coupling force between the two is decreasing. As shown in the figure, the stage will move to the right towards the negative bias. Coupling force between the stage and negative bias reaches a peak when the sinusoidal signal reaches its peak. As the intensity of the voltage reduces, the coupling force between the stage and right comb become weaker, the coupling force between the stage and the left comb becomes stronger, and the stage will shift back towards center. When the voltage of the sinusoidal signal reaches zero the voltage potentials between the stage the right and left combs are equal and the coupling forces upon the stage are equal, so the stage is held in center. As the voltage signal applied to the stage moves into the negative half of the cycle, coupling forces increase between the stage and the left comb, and decrease between the stage and the right comb, and the stage will shift in the opposite direction. The signal voltage will reach peak value and the stage will deflect to the farthest left. As the voltage signal reduces in intensity back towards a zero voltage, the stage will shift back to center [34]. 


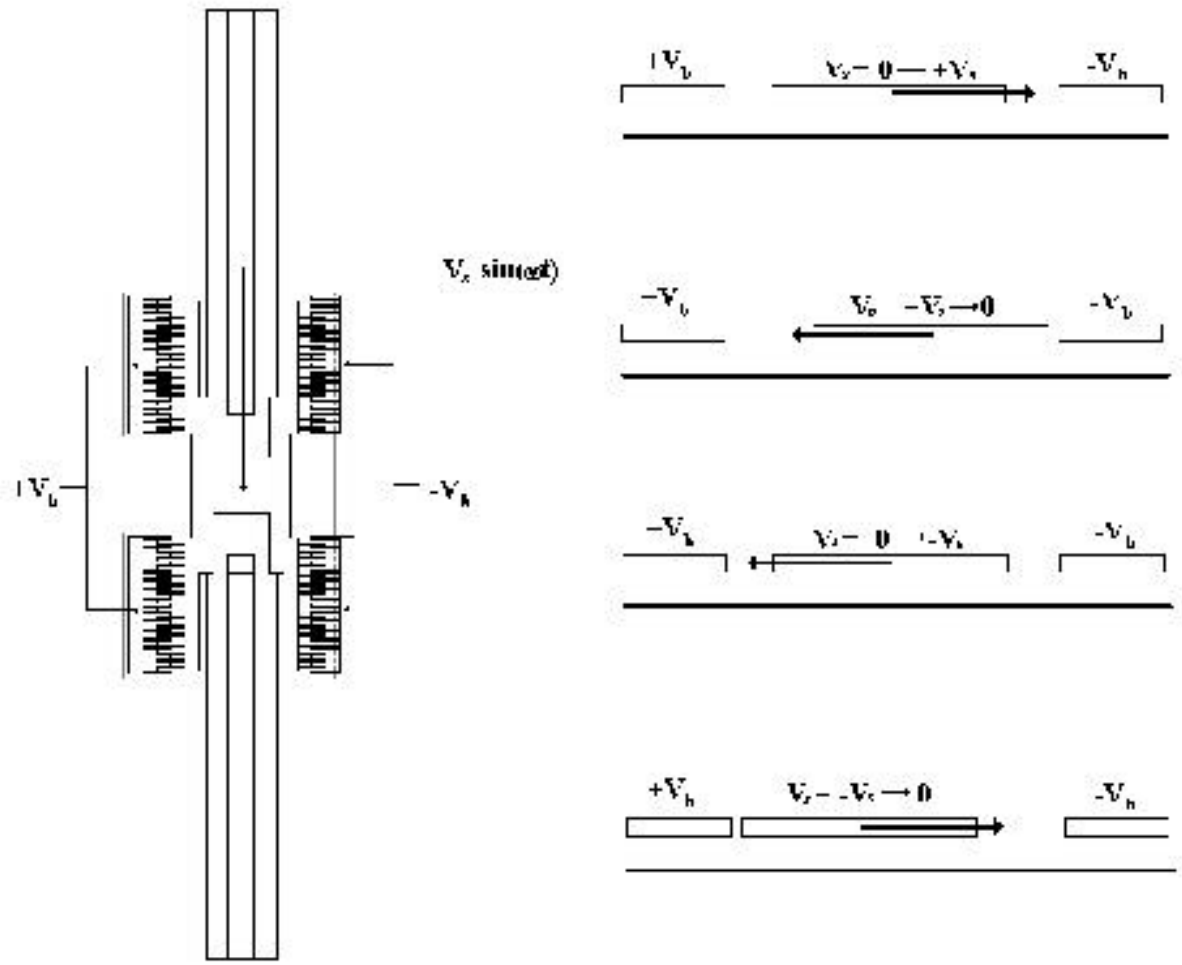

Figure 3-4: Typical Operation of a Linear Resonator MEMS Device

\subsection{Linear Resonator Forces Under Ideal Operation}

If we assume that there is no load applied to the linear comb stage, then the only forces that are exerted on the stage are those of the electrostatic forces created by the capacitive combs, the spring restoring force created by the folded flexure structures, and a friction arising from air resistance on the linear resonator stage. Together the system forms a driven harmonic oscillator with damping. A classic dynamic model of the lumped forces in the system is shown in Figure 3-5. To understand and predict the motion of the device, these forces will be examined individually. 


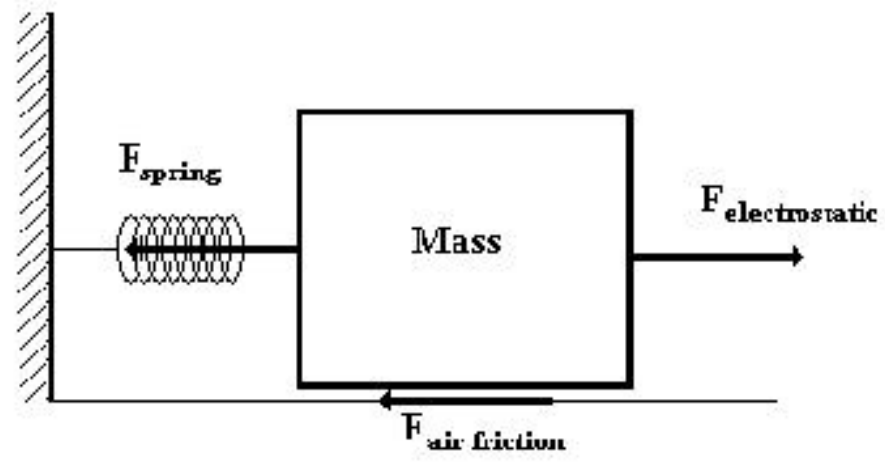

Figure 3-5: Dynamic Model of Lumped Force on Linear Resonator Device.

\subsubsection{Electro static Comb Forces}

The capacitive comb drive was developed by Tang and Howe. A close up of this structure is shown in Figure 3-6. Comb drives are a type of capacitive electrostatic actuator creates a force that remains linear over the range of motion. An electrostatic comb drive requires two comb structures. One comb must remain stationary and the other comb will be free moving. In Figure 3-6, the left comb is assumed to be the stationary one and the right comb is free-moving. The force that causes the free structure to move is created by the capacitance between the two structures. Inter-digitating the two structures increases the capacitive area, which increases the force [7, 34].

In this figure, the stationary comb structure is held at voltage, $\mathrm{Vb}$, and a signal voltage, $\mathrm{Vs}$, is applied to the free moving structure. The length of each comb finger is $\mathrm{L}$. The spacing between the 


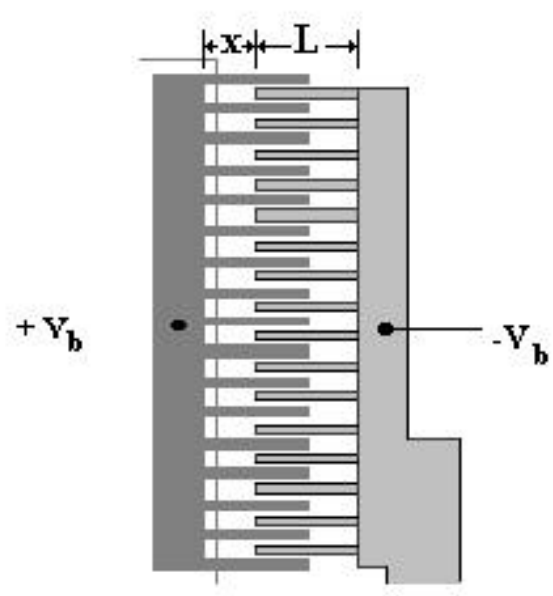

Figure 3-6: Close up of Resonant Comb Drive Structure

inter-digitated fingers is called the gap, g. The other gap spacing, that between the finger ends and the comb structure is labeled, $\mathrm{x}$. This is the distance between the two comb structures and it will also represent the displacement that the right comb will move.

The linear resonator stage is driven by two capacitive comb drives, one the left side of the device and one on the right side of the device. The following derivation of force that acts on the free moving stage is referenced for the comb structure on the left half of the device. It is assumed that the electric fields created by the flat faces of the finger tips, the electric fields that span the $\mathrm{x}$ gap, are negligible and the only significant electric fields are those which contribute to motion is the fields across the g gap. Therefore the capacitance between the two comb finger faces is

$$
C=\frac{\varepsilon A}{g},
$$


where is the permitivity of the material between the gap fingers and $A$ is the area that the fingers overlap [7].

The area can be calculated by multiplying the thickness of the MEMS devices, $t$, by the length of the overlap, L-x. Therefore,

$$
A=t(L-x)
$$

If there are n number of fingers on the free-moving comb structure then the amount of capacitive area is

$$
A_{\text {tot }}=2 n t(L-x)
$$

since each finger has two faces. The total capacitance of this comb structure is,

$$
C_{t o t}=\frac{2 n \varepsilon \cdot A}{g}
$$

[7].

To determine the force being applied to the free moving comb, the potential energy of the system must first be determined. The energy being stored in the electric field of the capacitor is related to the voltage across the capacitor, so

$$
w_{\text {field }}=\frac{1}{2} C\left(V_{s}-V_{b}\right)^{2}
$$

The energy being applied to the capacitive comb structure is

$$
w_{a p p}=-C\left(V_{s}-V_{b}\right)^{2} .
$$


Therefore the total potential energy in the system is

$$
w=w_{\text {field }}+w_{\text {app }}=-\frac{1}{2} C\left(V_{s}-V_{b}\right)^{2}
$$

Substituting in the capacitance derived in Eq. 3.4, the potential energy can be written as

$$
w=-\frac{n \varepsilon A}{g}\left(V_{s}-V_{b}\right)^{2}
$$

[35].

To determine the force exerted on the actuator, the principle of virtual work is used. Virtual work employs the idea that for very small changes in displacement, changes in the system's energy can be found, so that if no real work is applied to the system, i.e. virtual work, then for a virtual displacement the change in energy in the system can be found. To restate the principle of virtual work in mathematical form,

$$
\partial W=F \partial x-\partial w
$$

but,

$$
\partial W=0
$$

In this equation $M$ is the virtual displacement. So if we substitute the potential energy equation into Eq. 3.8 , then

$$
\partial W=F \partial x+\partial\left[\frac{n \varepsilon t(L-x)}{g}\left(V_{s}-V_{b}\right)^{2}\right]
$$


If the virtual work is set to zero, and factor out the virtual displacement the equation becomes

$$
0=\left[F+n \varepsilon \frac{t}{g}\left(V_{s}-V_{b}\right)^{2}\right] \partial x .
$$

Therefore the electrostatic force acting on the comb drive is

$$
F=n \varepsilon \frac{t}{g}\left(V_{s}-V_{b}\right)^{2} .
$$

[36].

In Eq. 3-12 the electrostatic force is the square of the voltage potential, as shown in Figure 37. The force created by one capacitive comb structure is non-linear, however the combination of the two capacitive comb drives will create a linear force relationship. (See section 3.4 below.)

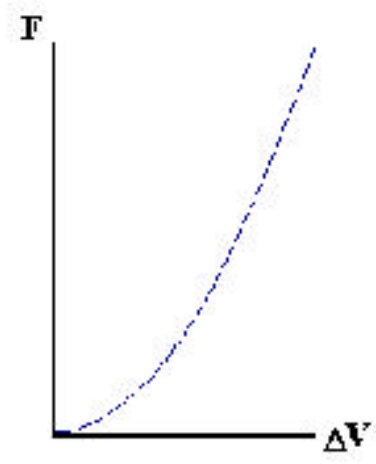

Figure 3-7: Force-Potential Voltage Curve 


\subsubsection{Folded Flexure Spring Forces}

The folded flexures support the resonator stage. They are also anchored to the ground plane. As the stage moves, the folded flexures bend, which creates a restorative force in the direction opposite to the motion. This force acts like a spring and can be represented by the equation,

$$
F=-k x
$$

where $\mathrm{x}$ is the magnitude of deflection and $\mathrm{k}$ is the spring constant.

Folded flexure structures are chosen to support the stage because they apply forces only in the direction of motion, $\mathrm{x}$. Simple beam supports would bend and deflect in both the $\mathrm{x}$ and $\mathrm{y}$ directions. The folded flexures produce a linear spring constant over small deflections. At very large deflections the spring constant of the flexure becomes non-linear, but in this paper it is assumed that the deflections are small enough that the spring constant, $\mathrm{k}$, remains linear $[18,37]$.

The equation for the spring constant is

$$
k=\frac{E a^{3} t}{l^{3}}
$$

Where E is Young's modulus of elasticity for polysilicon, $\mathrm{a}$ is the thickness of one spring beam, $\mathrm{t}$ is the thickness of the polysilicon layer, and 1 is the length of the spring [38]. By substituting Eq. 3.15 into Eq. 3.14, the final force equation can be written as,

$$
F=-\frac{E a^{3} t}{l^{3}} x
$$




\subsubsection{Air Resistive Damping Force}

The third force that acts upon the resonator device is a viscous damping force caused by the air around the resonator. Unlike the unrestricted air above the resonator surface, which can oscillate with the resonator, the air that is trapped under the stage is restricted. As the resonator moves it rides upon this pocket or bubble of air, that cannot move freely. This creates a shear friction on the undersurface of the resonant structure, which is sometimes referred to as a skin friction and it is related to the viscosity of the air $[15,39]$.

Because this friction opposes the direction of velocity, the damping force can be written as,

$$
F=-B \frac{\partial x}{\partial t} .
$$

Where, B is the damping coefficient, and is defined by the equation,

$$
B=\mu\left[\left(A_{s}+\frac{A_{t}}{2}+\frac{A_{b}}{2}\right)\left(\frac{1}{d}\right)+\frac{A_{C}}{g}\right],
$$

where $F$ is the viscosity of air, $A_{s}$ is the surface area of the stage, $A_{t}$ is the surface area of the folded flexure truss (the small beam that connects the two folded flexures), $A_{b}$ is the surface area of the beam and $A_{c}$ is the total surface area for the comb fingers. The constant $d$ is the distance between the ground plane and the undersurface of the resonator, and finally $g$ is the gap space between the comb fingers [15]. 


\subsection{Model of Linear Resonator Motion}

As stated above there are three forces which act upon an unloaded linear resonator: the electrostatic force, the spring force and the viscous damping force. Using Newton's second law of motion we will be able to calculate the expected displacement for the MEMS structure when it is given a specified electrical input. The electrostatic force equation is shown in Eq. 3.13. However, there are two comb drives so there will be two electrostatic forces added into the equation. The voltage potentials between the two combs are $\mathrm{V}_{\mathrm{s}}+\mathrm{V}_{\mathrm{b}}$ and $\mathrm{V}_{\mathrm{s}}-\mathrm{V}_{\mathrm{b}}$. When in operation, the forces caused by these two separate potentials will force the MEMS stage in the same direction, because one will be attractive and one will be repulsive. When mathematically representing these forces, the sign of direction of motion will be applied in the attractive phase,

therefore the attractive force caused by the positive voltage bias will be,

$$
F_{1}=n \varepsilon \frac{t}{g}(V s-V b)^{2},
$$

and the force cause by the negative voltage bias will be,

$$
F_{2}=-n \varepsilon \frac{t}{g}(V s+V b)^{2} .
$$

The cumulative electrostatic forces will be,

$$
F_{\text {tot }}=n \varepsilon \frac{t}{g}\left(V_{s}-V_{b}\right)^{2}-n \varepsilon \frac{t}{g}\left(V_{s}+V_{b}\right)^{2} .
$$


Expanding out the equation results in,

$$
F_{\text {elec. }}=n \varepsilon \frac{t}{g}\left[\left(V_{s}^{2}-2 V_{s} V_{b}+V_{b}^{2}\right)-\left(V_{s}^{2}+2 V_{s} V_{b}+V_{b}^{2}\right)\right]
$$

Simplifying the equation results in,

$$
F_{\text {elec. }}=-4 n \varepsilon \frac{t}{g}\left(V_{b} V_{s}\right)
$$

The cumulative electrostatic force created by both capacitive comb structures is a linear relationship between the force and the voltage potential. In Eq. $3.23 \mathrm{~V}_{\mathrm{s}}$ is the driving signal, and it takes the form of $\mathrm{V} \cos (? \mathrm{t})$, so equation 3.23 can be rewritten as,

$$
F_{\text {elec. }}=-4 n \varepsilon \frac{t}{g}\left(V_{b} V\right) \cos (\omega t) \text {. }
$$

If we substitute,

$$
F_{o}=-4 n \varepsilon \frac{t}{g}\left(V_{b} V\right) \text {, }
$$

then the electrostatic force equation is simplified to,

$$
F_{\text {elec. }}=F_{o} \cos (\omega t)
$$


Eq. 3.14 represents the spring force for one folded flexure. Since there are two flexures on each device, the total spring force in the system is

$$
F_{\text {spring }}=-2 k x \text {. }
$$

Newton's second law states,

$$
\Sigma F=m a
$$

$[37,40]$. Summing the three force equations: $3.26,3.27$ and 3.17 , the newton force equation is

rewritten as,

$$
F_{0} \cos (\omega t)-2 k x-B v=m a .
$$

Rewriting the acceleration and velocity as derivations of $\mathrm{x}$ with respect to time, the force equation is

$$
F_{0} \cos (\omega t)-2 k x-B x^{\prime}=m x^{\prime \prime} .
$$

Rearranging the equation yields

$$
m x^{\prime \prime}+B x^{\prime}+2 k x=F_{0} \cos (\omega t) .
$$

Equation 3.31 is a linear differential equation and that of the well known forced harmonic oscillator with damping. If the electrostatic force equation is rewritten as,

$$
F_{\text {elec. }}=\operatorname{Re}\left\{F_{o} e^{j(\omega t)}\right\}
$$

it can be assume a solution for $\mathrm{x}$ that takes the form,

$$
x=\operatorname{Re}\left\{A F_{0} e^{j(\omega t-\theta)}\right\},
$$

where $\mathrm{A}$ is a constant and ? is a phase shift between the displacement and the driving signal. 
The displacement may be written then as,

$$
x=A F_{0} e^{j(\omega t-\theta)} .
$$

The first derivative of $\mathrm{x}$ will be,

$$
x^{\prime}=j \omega A F_{o} e^{j(\omega t-\theta)},
$$

and the second derivative will be,

$$
x^{\prime \prime}=-\omega^{2} A F_{o} e^{j(\omega t-\theta)} \text {. }
$$

Substituting the derivatives into Eq. 3.31,

$$
\begin{aligned}
& -m \omega^{2} A F_{o} e^{j(\omega t)} e^{-j \theta}+j B \omega A F_{o} e^{j(\omega t)} e^{-\lambda \theta}+2 k A F_{o} e^{j(\omega t)} e^{-\lambda \theta} \\
& =F_{0} e^{j(\omega t)}
\end{aligned}
$$

Simplifying the equation by dividing out and factoring common elements,

$$
A e^{-\lambda \theta}\left(-\omega^{2} m+j \omega B+2 k\right)=1 .
$$

Solving the equation for $\mathrm{Ae}^{-\mathrm{j} \text { ? }}$,

$$
A e^{-\lambda \theta}=\frac{1}{-\omega^{2} m+j \omega B+2 k} .
$$


The final solution is found by solving for the real parts of the displacement coefficient, A, and the phase shift, ?, in terms of the force equation coefficients and the frequency. So,

$$
\operatorname{Re}\{A\}=\frac{1}{\left[\left(2 k-\omega^{2} m\right)^{2}+(\omega B)^{2}\right]^{\frac{1}{2}}},
$$

and,

$$
\operatorname{Re}\{\theta\}=\tan ^{-1}\left(\frac{\omega B}{2 k-\omega^{2} m}\right) .
$$

\subsection{Resonant Frequency Calculation}

Resonance is the natural frequency of a system, and it is calculated with the assumption that the damping force is zero $[37,40]$. At resonance, without damping, the deflection of the linear resonator will go to infinity. The equation for displacement as shown in Eq. 3.34 has two coefficients which determine the amplitude. $\mathrm{F}_{\mathrm{o}}$ is the input signal amplitude which is constant. However, $\mathrm{A}$, the amplifying coefficient, is a function of ? Remembering that $B=0$, we can set $A$ to 4 , which results in the equation:

$$
\infty=\frac{1}{\left(2 k-\omega^{2} m\right)^{2}} .
$$


Inverting both sides of the equation yeilds,

$$
0=2 k-\omega^{2} m
$$

and solving for frequency,

$$
\omega=\sqrt{\frac{2 k}{m}} .
$$

Eq. 3.44 is the equation to find the resonant frequency of the linear resonator MEMS device.

If the resonant frequency, is inserted in the phase shift equation, Eq. 3.41,

$$
\theta=\tan ^{-1} \frac{\omega B}{2 k-\left(\sqrt{\frac{2 k}{m}}\right)^{2} m}
$$

The denominator in the above equation equals zero, therefore the phase shift is equal to $90 \mathrm{E}$ at resonance.

\subsection{Calculated Frequency for a 550 $\mu \mathrm{m}$ Flexure Length Linear Resonator}

Table 3-1 displays the physical characteristics and properties values for the linear resonator displayed in Figure 3-2. There are over 16 MEMS devices on one die, and all of the ten resonators in the middle of the die, were fabricated specifically for integrated waveguide co-integration. For each of the ten resonators, the device stages were made with the same dimensions and the length of the folded 
flexures was varied from device to device. All of the optical measurements taken in the next chapter, were performed on the two linear resonator devices with $550 \mathrm{Fm}$ flexures.

Calculating resonance of the linear resonator with $550 \mathrm{Fm}$ flexure length requires solving for the three different coefficients in Eq. 3.40 and 3.41: m, k, B. The mass of each stage element was found by multiplying the volume of each individual element and multiplying it by the density, ?. The total stage mass was calculated using the equation:

$$
m=m_{\text {stage }}+\frac{1}{4} m_{\text {truss }}+\frac{12}{35} m_{\text {beams }} .
$$

This equation weights the mass of the beams, and their contribution to motion in the $\mathrm{x}$ direction. The total mass of the stage was found to be $222.7 \times 10^{-12} \mathrm{Kg}$. The spring constant, $\mathrm{k}$, was solved for using Eq. 3.15. I has a value of $15.6 \times 10^{-3}$ Pa-m. Eq. 3.18 was used to calculate the damping coefficient: $\mathrm{B}=0.416 \times 10^{-6} \mathrm{Kg} / \mathrm{s}^{2}$.

Substituting these coefficients into the resonant frequency equation, Eq. 3.44, yields a resonant frequency, ? ${ }_{\mathrm{r}}$, equal to $11.896 \times 10^{3} \mathrm{Krad} / \mathrm{s}$. If this number is divided by $2 \mathrm{p}$, the resonant frequency in hertz is $\mathrm{f}_{\mathrm{r}}=1.89 \mathrm{KHz}$. Equation 3.40 and 3.42 can be graphed as a function of the frequency. The change in amplitude as a function of the frequency is shown in Figure 3-8. In the graph, amplitude peaks at $1.89 \mathrm{KHz}$. This peak is very narrow, which indicates that the damping coefficient is low. Figure 3-9 plots the phase shift as a function of frequency. Notice the phase shift reaches $90 \mathrm{E}$ at the resonant frequency and then inverts to $-90 \mathrm{E}$ 


\begin{tabular}{|l|l|r|}
\hline $\mathrm{t}$ & Thickness of polysilicon layer & $2 \mu \mathrm{m}$ \\
\hline 1 & Length of folded flexure & $550 \mu \mathrm{m}$ \\
\hline $\mathrm{a}$ & Width of folded flexure & $2 \mu \mathrm{m}$ \\
\hline $\mathrm{A}_{\mathrm{b}}$ & Surface area of folded flexure & $8,800 \mu \mathrm{m}^{2}$ \\
\hline $\mathrm{A}_{\mathrm{t}}$ & Surface area of truss & $408 \mu \mathrm{m}^{2}$ \\
\hline $\mathrm{L}$ & Length of comb fingers & $40 \mu \mathrm{m}$ \\
\hline $\mathrm{A}_{\mathrm{c}}$ & Total surfae area of comb fingers & $10,240 \mu \mathrm{m}^{2}$ \\
\hline $\mathrm{n}$ & Number of comb fingers & 32 \\
\hline $\mathrm{g}$ & Gap size between comb fingers & $2 \mu \mathrm{m}$ \\
\hline $\mathrm{A}_{\mathrm{s}}$ & Area of stage & $34,440 \mu \mathrm{m}^{2}$ \\
\hline $\mathrm{d}$ & Distance between ground plane and stage & $2 \mu \mathrm{m}$ \\
\hline $\mathrm{g}$ & Permitivity of air & $8.854 \times 10-12 \mathrm{~F} / \mathrm{m}$ \\
\hline $\mathrm{E}$ & Young's modulus of polysilicon & $165 \mathrm{GPa}$ \\
\hline$\mu$ & Viscosity of air & $16.9 \times 10^{-6} \mathrm{~N}-\mathrm{s} / \mathrm{m}^{2}$ \\
\hline$?$ & Density of polysilicon & $2330 \mathrm{Kg} / \mathrm{m}^{3}$ \\
\hline
\end{tabular}

Table 3-1: Linear Resonator Physical Characteristics and Properties [15, 35, 37, 38]. 


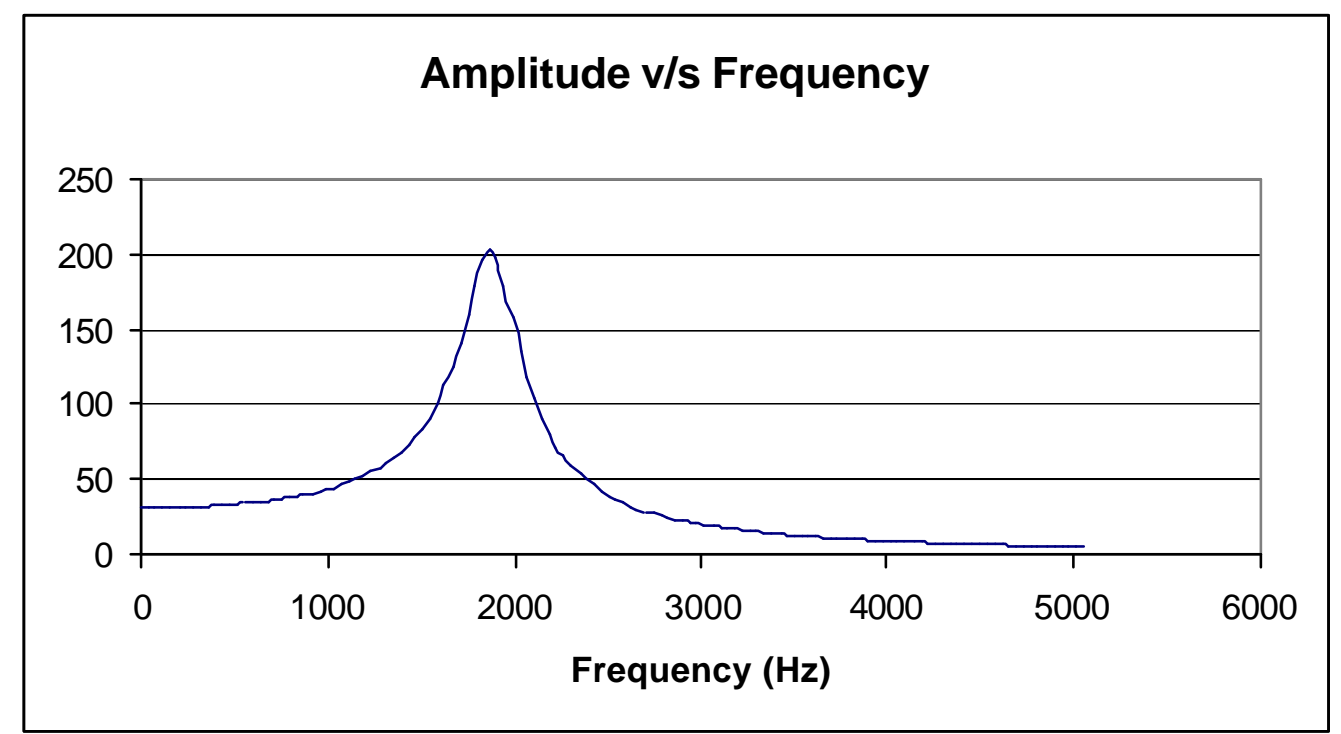

Figure 3-8: Frequency Plot of Amplitude of 550 Fm Flexure Length Linear Resonator

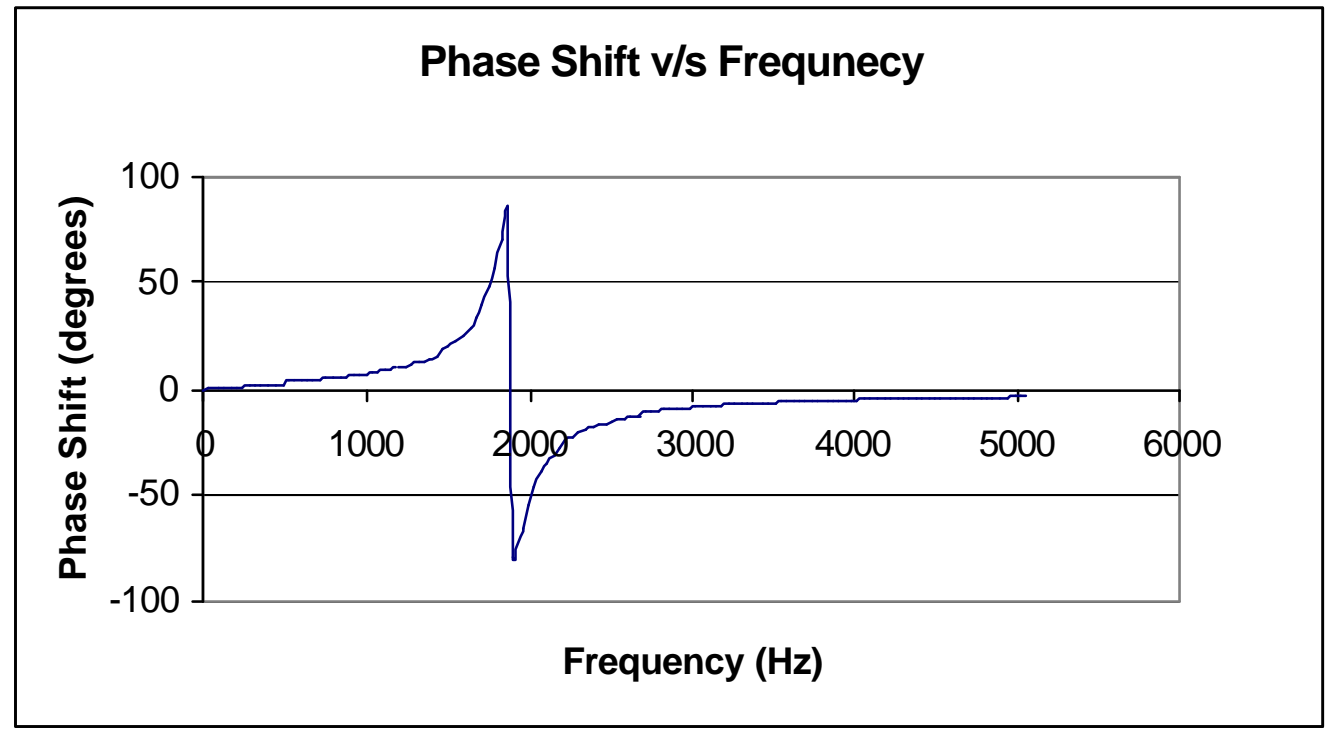

Figure 3-9: Frequency Plot of Phase Shift of 550 Fm Flexure Length Linear Resonator 


\section{Chapter 4}

\section{Bulk Optical Metrology}

The prefabricated linear comb resonator presented in the previous chapter has a calculated resonant frequency of $1.89 \mathrm{KHz}$. Physically the maximum allowable deflection is $\pm 20 \mathrm{Fm}$, although the device does not achieve these distances during operation. To characterize the comb driven, linear resonator, a method is needed that can measure the device operating frequency, which can range from less than $1 \mathrm{~Hz}$ to several $\mathrm{KHz}$. Motion over a range of $40 \mathrm{Fm}$ must be detected. Besides measuring the frequency and deflection, it is also desirable to measure the phase shift between the motion of the device and the input drive signal.

Measuring the deflection, frequency, and phase shift using an optical signal requires illuminating the edge of the linear resonator stage, and measuring the intensity of the light reflected back. Two different optical phenomenon can create changes in the optical feedback intensity. The first is divergence. As the resonator moves back and forth, the optical path length increases and decreases. Because the optical input signal will diverge over distance, the intensity of the light decreases as the optical path length increases. The second phenomenon is interference. If the optical source is coherent, then the reflected light will interact with the input light, creating constructive and destructive 
interference. The optical output signal will vary in intensity, depending on the amount of interference created by the phase shifts in light, which are dependent on the optical path length.

To perform these optical measurements, a way must be found to introduce light from a source to the resonator stage edge, and a way must be found to collect the light reflected by the MEMS device and translated to an optical detector. The goal of this research is to explore the use of integrated optical waveguides to perform these functions. An initial study of the optical measurement was needed before attempting to integrate optical waveguides with MEMS structures. The best obtainable approximation of the integrated waveguides was to use optical fibers to perform the functions of introducing light for illuminating the resonator stage edge, and for collecting light from reflected from the resonator for optical detection. Both fiber optic measurement and integrated waveguide measurement methods will utilized guided wave optics to introduce light onto the device, and the BOM measurement will attempt to see if the reflected signal can be captured using an optical fiber.

While not integrated optical metrology, the experiment presented in this chapter will show the feasibility of using guided waves for MEMS metrology. Optical fiber probing can be used to characterize the linear resonator device experimentally, with results that closely match the behavior theoretically predicted in the previous chapter. The experiments will also examine the different optical phenomenon used to detect linear motion of the resonator by exploring both multimode and single mode fiber probing. These issues will need to be addressed when using guided wave optics, regardless of using optical fibers or integrated waveguides. This chapter will present the experimental setup, the measurements and the discussion of results. 


\subsection{Experiment Objectives}

The basis of this experiment is to assess the feasibility of using optical waveguides to characterize a MEMS device by measuring the motion of a linear resonator device and its parameter. The same device that was designed for integration of optical waveguides in this dissertation will be used for the optical fiber experiments. Linear resonators are deformable MEMS devices that move in a lateral motion parallel to the plane of the substrate. The previous chapter has presented the theoretical, electro-mechanical characteristics of linear comb resonators. During the measurement process the objectives are to see which of these characteristics can be detected. Of particular interest, are the detection of motion, displacement, operating frequency, resonant frequency, and phase shift, using multimode and single mode optical waveguides.

Bulk optical metrology (BOM) experiments undertaken require the use of two individual optical fibers. The input fiber is coupled to a laser, and it is used to illuminate the surface of the object that is moving. The optical signal measurements using the optical fibers were performed twice, once using a single mode input fiber and again using a multimode input fiber. By repeating the experiment with a different input fiber, the measurements taken reflect the two methods of detecting motion, interference and intensity. Single mode fibers have a small core diameter, approximately $8 \mathrm{Fm}$, and support only the primary mode. The higher order modes, which are not supported, are radiated from the fiber. However, the single mode produces a single output intensity field mode and there is no interference speckle pattern which occurs when there are multiple modes; meaning all diffraction patterns created are the result of the reflected signal. Having large core diameters, multimode fibers are easier to couple power into and when fully moded carry more optical power. Therefore the multimode input fiber is 
expected to produce a stronger input signal to the device than the single mode fiber. The disadvantage

of multimode fibers is the speckle pattern created by the interaction of modes. A second advantage of the multimode fibers is that they produce larger spot sizes, which will make alignment of the detector fiber easier.

The second fiber, referred to as the output fiber, is used to collect the light reflected off the object that is moving and carry it to an optical detector. In both experiments the ease of alignment and large intensity gained by using a multimode detector fiber was significant, so a multimode detector fiber was used in both measurement experiments.

\subsection{Bulk Optical Metrology Experimental Setup}

A setup for a BOM optical fiber measuerment is shown in Figure 4-1. A $60 \mathrm{~mW}$ He-Ne laser (optical wavelength of $632.8 \mathrm{~nm}$ ) was used as a light source. This laser was coupled into the input fiber by focusing the laser beam onto the end-face of the fiber using a 20/0.050 microscope objective. A multimode fiber with core diameter of $98 \mathrm{Fm}$ and a cladding diameter of $140 \mathrm{Fm}$ was used for the input fiber for the multimode, and a single mode fiber with a core diameter of approximately $8 \mathrm{Fm}$ and a cladding diameter of $125 \mathrm{Fm}$ was used for the input fiber in the single mode experiments. Both fibers were bare fibers cut from a reel and all the end faces were cleaved by hand.

The input and output fibers were attach to probe manipulators in a micro-electronic probe station to align and hold the fibers in position under a microscope. One end of the detector fiber 


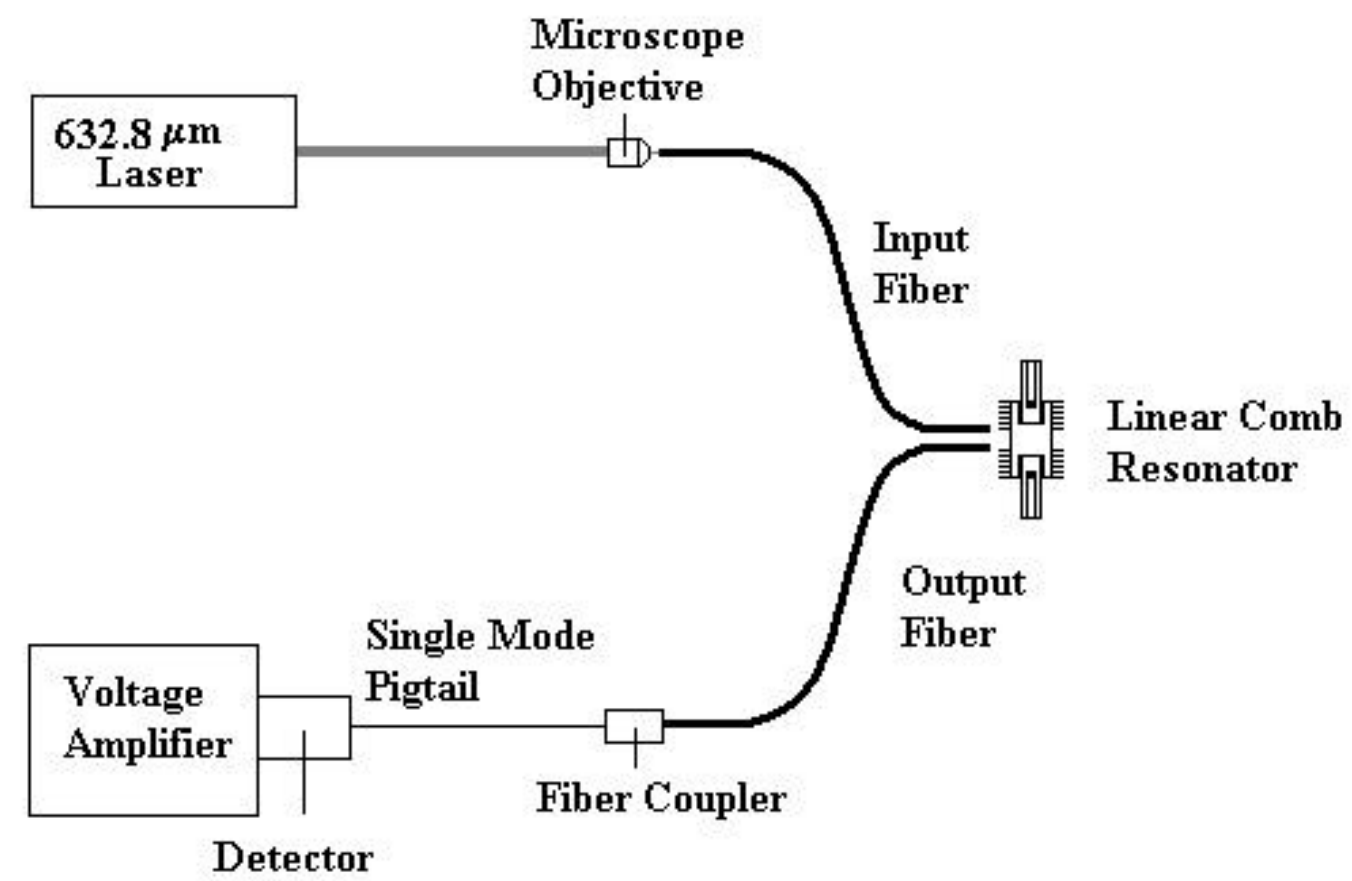

Figure 4-1: Fiber Optic Probe Setup for Bulk Optical Metrology

was cleaved by hand and was used to collect the light reflected off of the linear resonator. The other end was polished and mounted in a coupler holder which was connected to a single mode fiber pigtail which is permanently connected to a photo-detector, which in turn is connected to a voltage amplifier.

\subsection{Optical Measurement of Displacement of a Large Reflective Surface}

Detection of the reflected optical signal will measure the displacement of the linear resonator, since the change in feedback intensity is a function of optical path length. A gap was designed in the linear resonator comb structures so that at flat surface would exist along the edge of the resonator stage for optical measurement. This reflective area is small, $2 \mathrm{Fm} \times 64 \mathrm{Fm}$, and the reflectivity of the 
polysilicon is low. Polysilicon has an index of refraction of 3.5, which yields a reflectivity of $31 \%$ at an air to polysilicon boundary.

Before trying to measure the motion of a linear resonator device, the displacement of a large, highly reflective surface will be measured using a multimode input fiber and multimode output fiber. To create a large, reflecting surface that can be positioned, a blunt end of a probe tip holder held in a probe manipulator was used. The surface of the probe holder end is much larger than the fibers. Basic alignment of these three items are shown in Figure 4-2. The purpose of this measurement was to find the appropriate alignment of the optical fibers with respect to the reflecting surface and to create an output curve which compares the detected optical intensity with respect to the displacement.

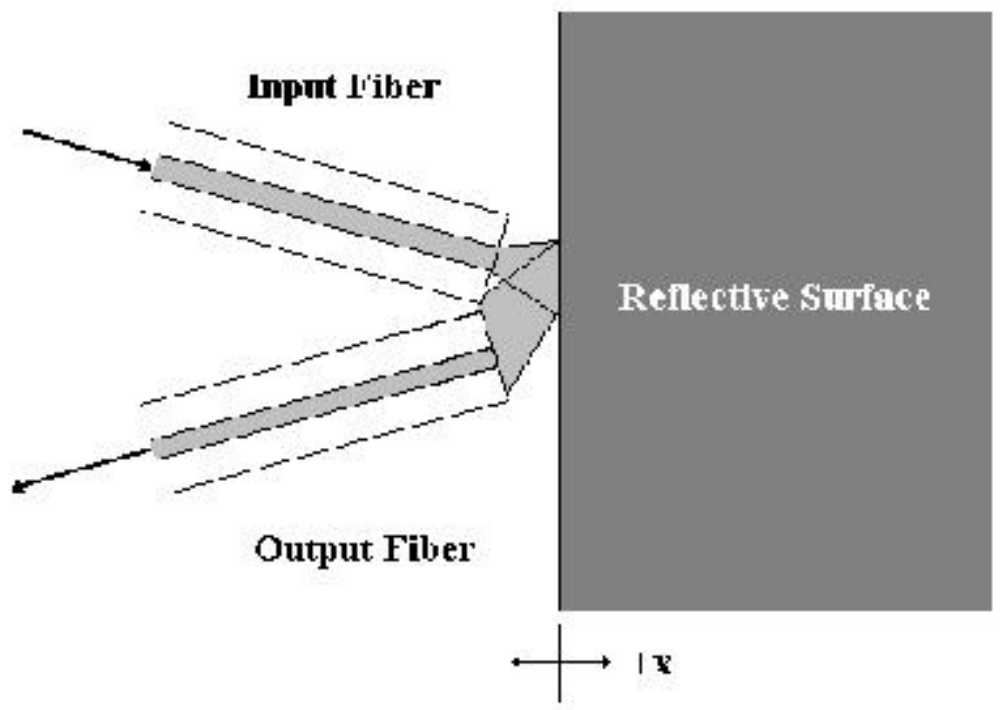

Figure 4-2: Multi-mode Fiber Alignment 
Because there was no initial reference, the zero position was assumed to be the point at which the reflecting surface touches the fiber optic tips, and the reflecting surface moved away from the initial starting in the positive $\mathrm{x}$ direction. To measure the distance that the reflective surface is moved, a digital linear gauge behind the probe manipulator. The positioning of this mounting is shown in Figure 4-3. Change in position is measured using the digital linear gauge by detecting the change in position of a telescoping spindle which collapses in itself, and converting the position change to an electrical signal. This measurement device has a resolution of $1 \mathrm{Fm}$ and has an overal range accuracy of $2 \mathrm{Fm}$.

Changes in optical intensity were measured by connecting a voltmeter to the output of the voltage amplifier connected to the optical detector. The DC voltage was recorded at each position measured. Measurements were taken over a range of $300 \mathrm{Fm}$. The plotted curve of this data is shown in figure 4-4.

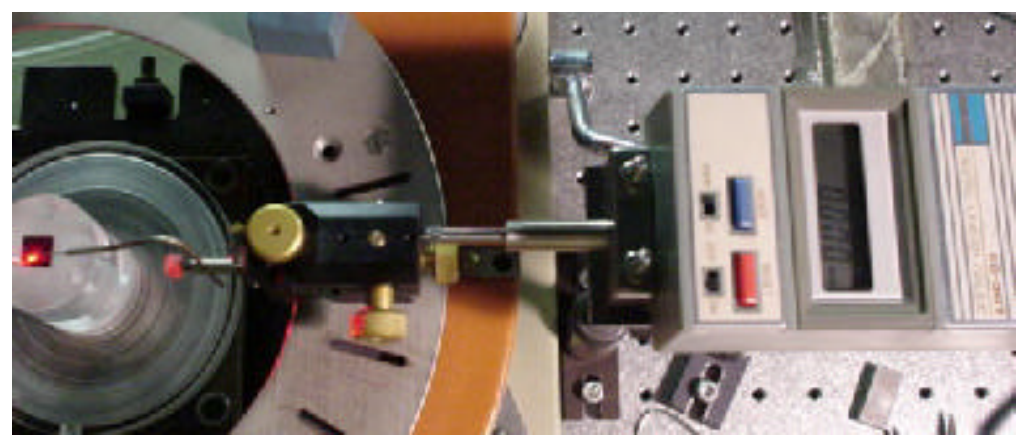

Figure 4-3: Position of Digital Linear Gauge 


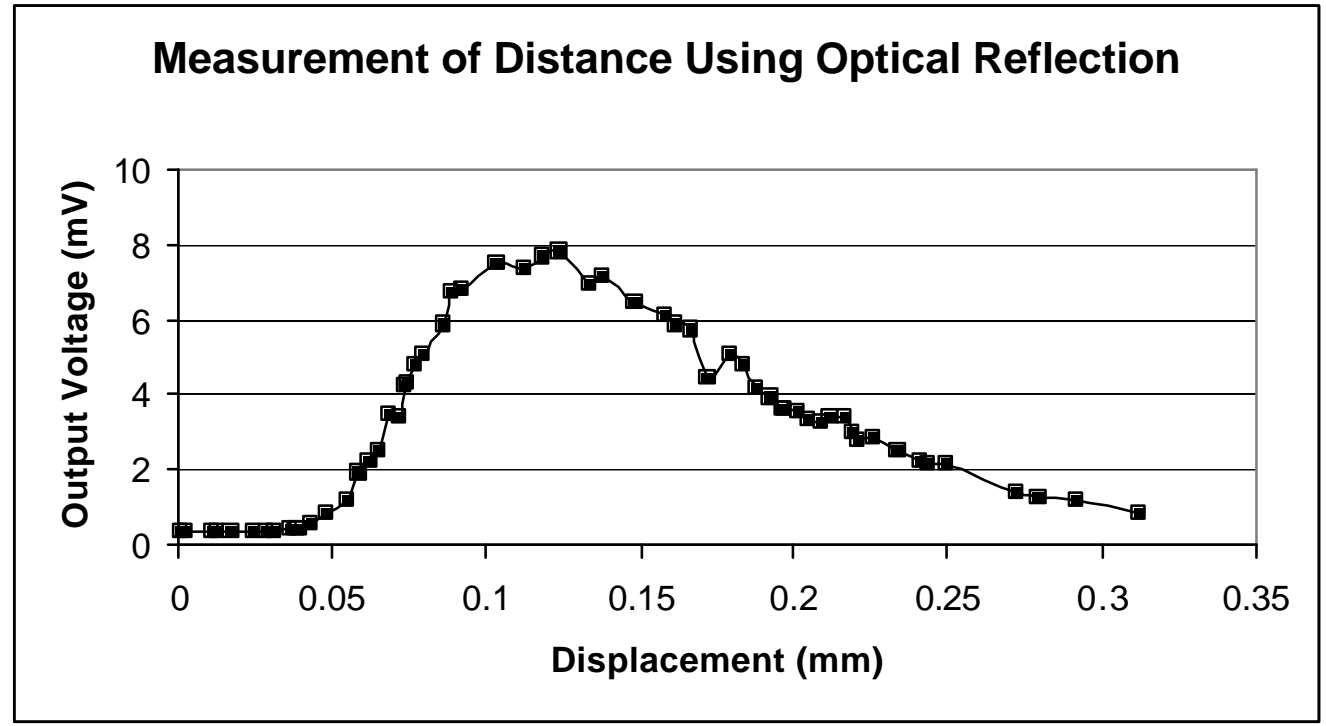

Figure 4-4: Optical Measurement of Displacement Using a Multimode Input Fiber and Multimode Detector Fiber

At the start of the graph (the range below $0.05 \mathrm{~mm}$ ) in Figure 4-5, the output voltage is very low. In this distance range the reflective surface is too close for the fiber, as shown in Figure 4-4a. Very little of the reflected light is collected by the detector fiber. After about $50 \mathrm{Fm}$ the optical signal increases very quickly. The graph peaks around $0.125 \mathrm{~mm}$, when the largest amount of optical power is coupled into the detector fiber as shown in Figure 4-5 b. Afterwards the output voltage slowly decreases as the light diverges and less of the optical power cross-section is coupled into the detector fiber. Figure 4-5 c and Figure 4-5 d show the divergence as the reflective surface is moved away from the optical fibers. 
a)

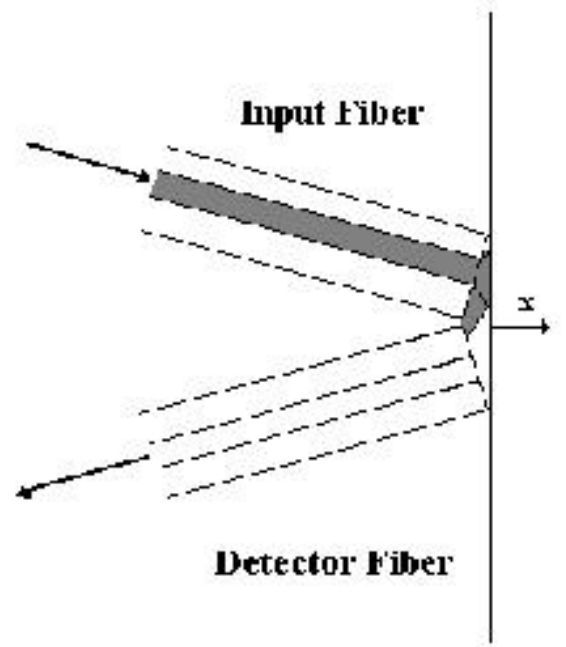

c)

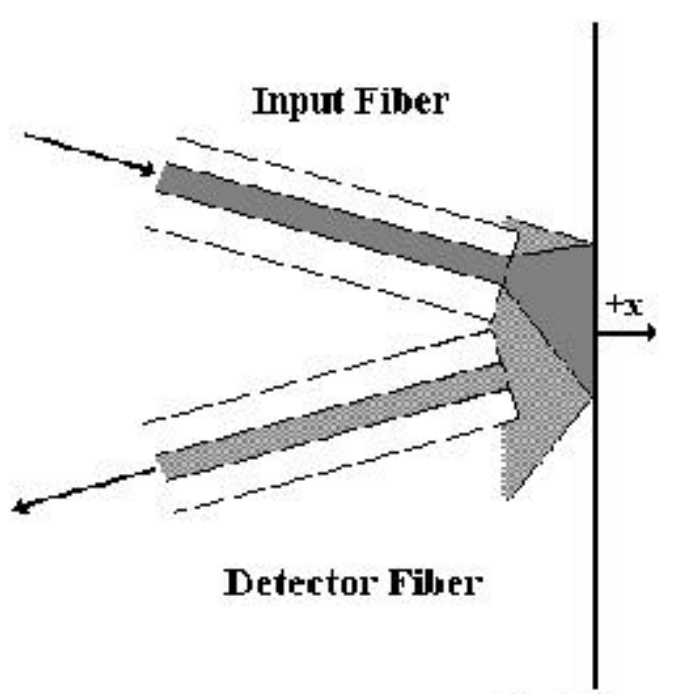

$\mathrm{x}-200 \mu \mathrm{m}$ b)

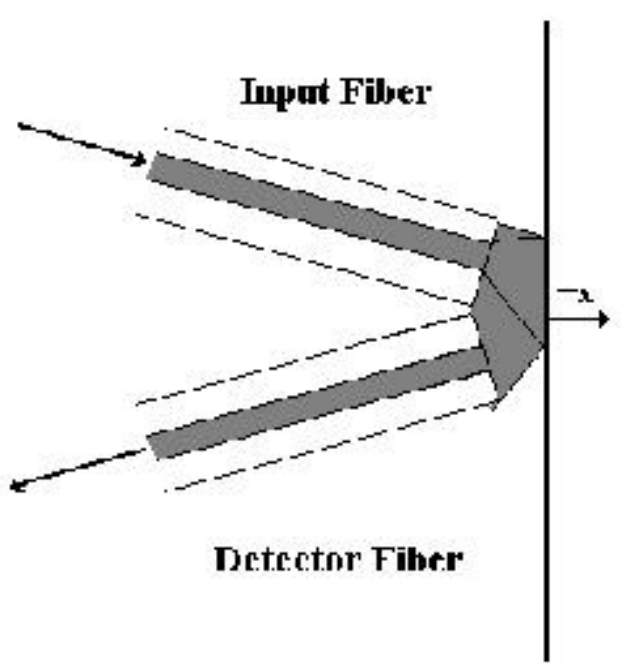

d)

$x \sim 100 \mu \mathrm{m}$

Figure 4-5: Optical Displacement Measurement of Large Reflecting Surface 


\subsection{Measuring the Displacement of a Linear Resonator Stage}

The previous measurement was used to establish the positional measurement capability and alignment of this optical fiber apparatus. Now the setup will be used to determine the displacement of a linear resonator. The edge of the linear resonator is much smaller, only $2 \mathrm{Fm}$ thick, and not perfectly reflecting. Positioning both the input and detector optical fibers will be important to maximize the output signal. Because the detection signal is likely to be small, and because the displacement less than

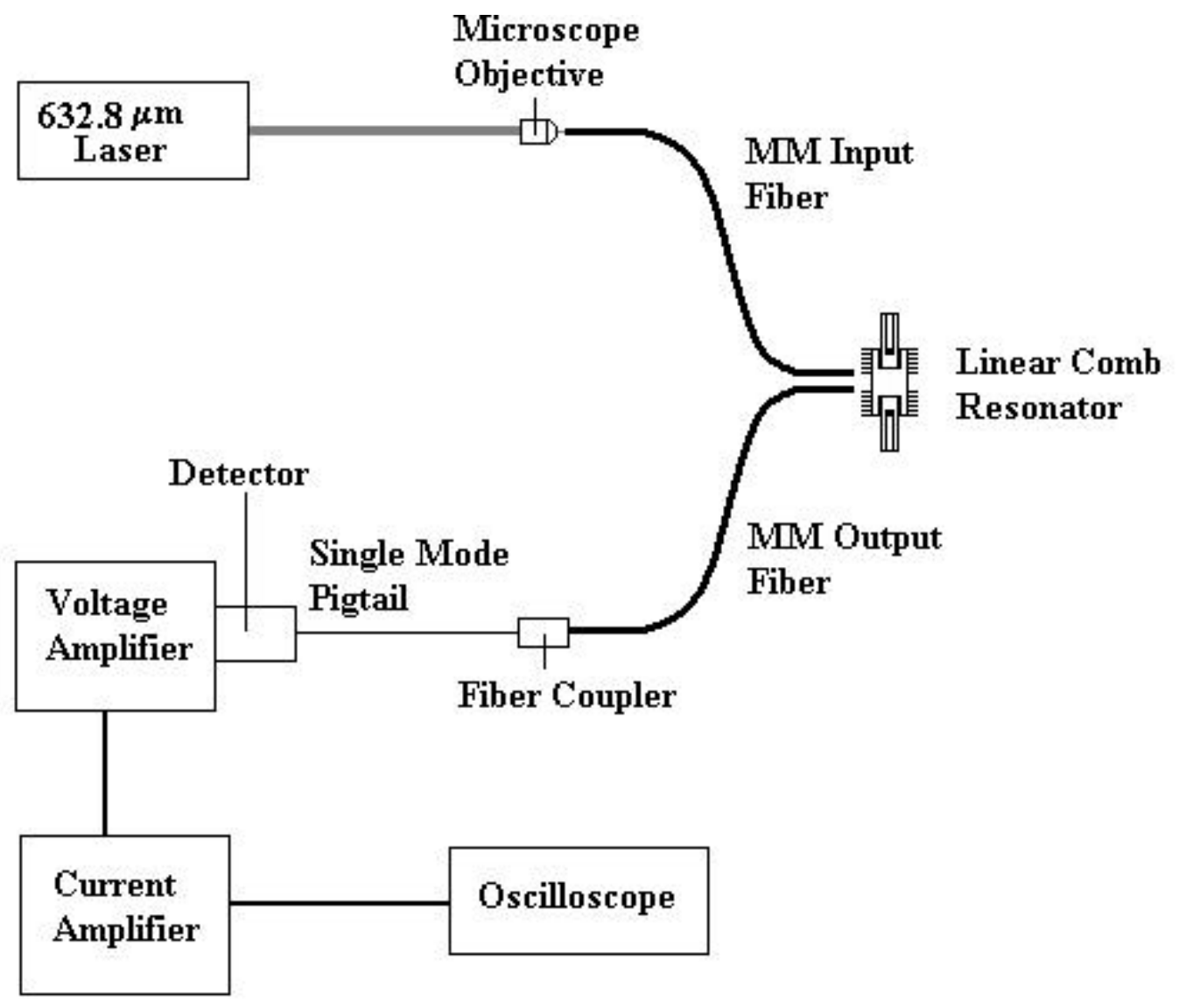

Figure 4-6: Experimental Setup for Measuring Linear Resonator Displacement and Motion 
$40 \mathrm{Fm}$, the output signal will need to be amplified. A diagram of the experimental setup is shown in Figure 4-6.

The die with fully released linear resonator devices is anchored in place in a probe station using a vacuum chuck. The same multimode input fiber is used as in the earlier experiment. The end of the optical fiber probe which outputs the laser power is attached to the tip of a probe, so that the fiber can be manipulated and positioned near the device without damage the fiber or the device. Because the optical losses were expected to be high, the amount of light being coupled into the input fiber had to be maximized. This process first required maximization of the coupling by adjusting the position of the fiber end face relative to the microscope objective being used to focus light onto the waveguide. This was achieved by visually inspecting the output intensity of the input fiber, which was shown upon a white sheet of paper. After maximization, the input fiber and the detector fiber were aligned using the probe manipulators. A chopper was inserted in the laser beam path, and the output of the detector fiber was coupled to an oscilloscope. The signal produced a square wave on the oscilloscope screen. To maximize the input coupling, fine adjustment was aided by adjusting the probe manipulators to maximize the square wave amplitude on the oscilloscope screen. The second step was to re-tune the position of the fiber end face to the microscope objective for maximum power coupling.

The same multimode detector fiber used in the earlier experiments was used, attached to the end of a probe manipulator to carefully position it next to the linear device. The alignment of this fiber was performed with the device in motion to maximize the output signal. Because both fibers were being introduced onto the resonator stage using the electronic probe setup, they were introduced at an angle 
to the die surface. The measurements were, therefore, out of the plane of the linear resonator by an angle of approximately $20 \mathrm{E}$.

Initially the linear resonator was moved by pushing its stage with a probe tip. Measurements were taken of this movement to establish the presence of any signal feedback difference. After a signal was established the linear resonator was moved using electrostatic actuation. Two types of measurements were be made. The device could be moved using either a static DC control signal or a sinusoidal signal. When using a DC voltage signal to move the device the, linear stage displaces to the right or left depending on signal polarity. The distance of motion is determined by the intensity of the DC voltage. Measurements were taken using the static displacement signal for determining the sensitivity of the optical measurement method to determine MEMS motion. The second measurement method used a sinusoidal control signal. When an AC voltage is applied to the linear resonator stage the device will oscillate at the frequency of the AC signal. Optical measurements were made to see if the displacement of the linear resonator could be detected. The output signal was compared to the input voltage signals to compare frequency and phase shift of the signal. Using both the phase shifts of the signal and the changes in the amplitude as the signal frequency was varied, the resonant frequency was determined.

\subsection{Forced Linear Resonator Displacement Measurement}

Before trying to measure an actuated device, it was important to measure the displacement of

the device. This measurement will determine what level of voltage variation will occur over a measured deflection. To measure the distance the linear resonator is displaced, the digital linear gauge was once 
again positioned against the probe manipulator. Whereas the earlier experiment used the blunt end of a probe holder, this experiment placed a probe tip in the probe holder and mounted it into the probe manipulator. This probe tip pushed against the edge of the linear resonator. A picture of the fiber positions and the probe tip is shown in Figure 4-7. The two optical fiber probes were positioned on the left of the device and the probe tip was positioned on the right.

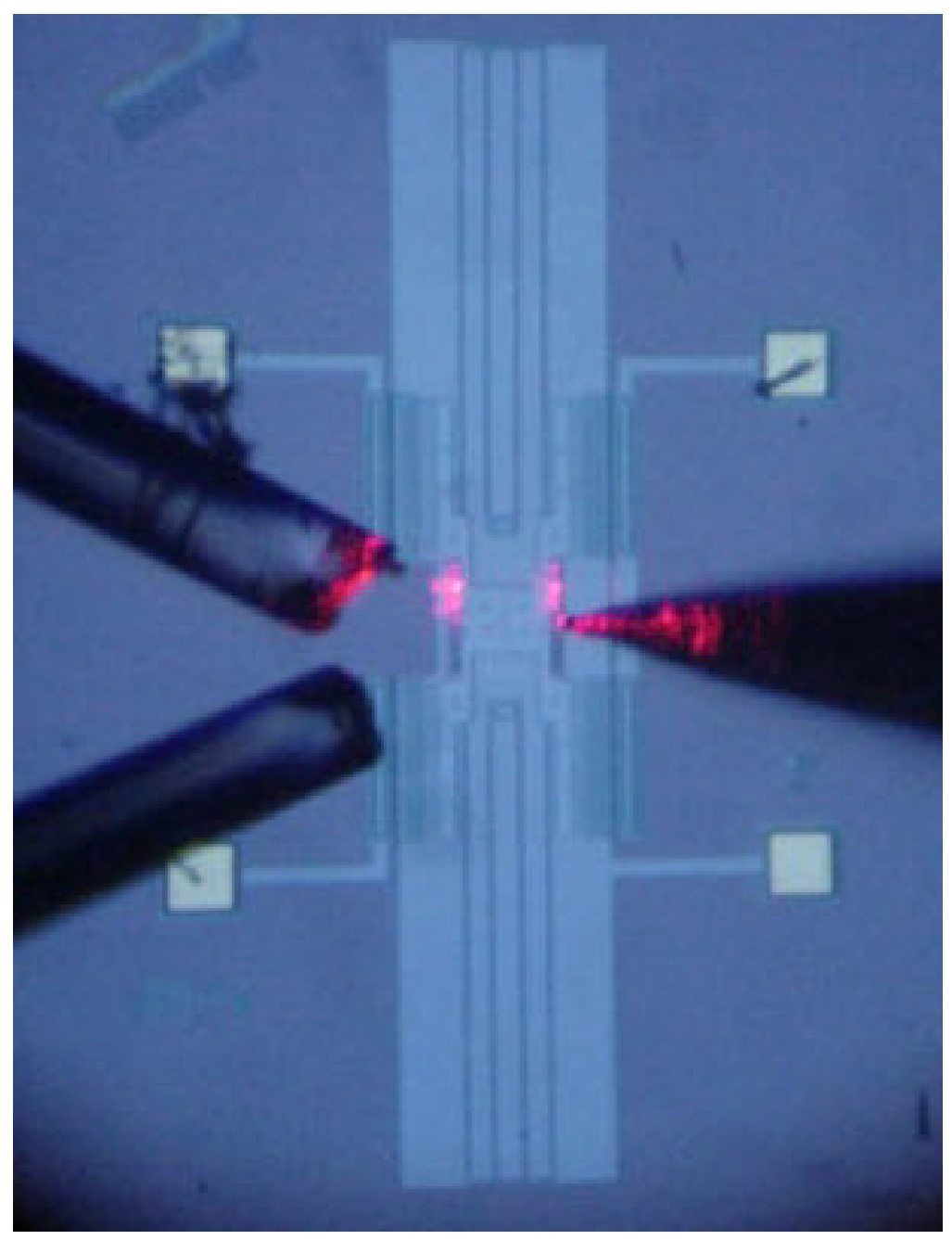

Figure 4-7: Fiber and Probe Alignments for the Multimode Fiber Forced Displacement Measurement 
As stated earlier, the fibers were aligned while the linear resonator was activated. The resonator chosen for measurement for the multimode trials had a flexure length of $550 \mathrm{Fm}$, and it also had extra structures built on the surface of the resonator stage, which were originally added to add vertical height to the cross-section of the resonator and increase the reflective surface area. The resonator was actuated by applying a $+8 \mathrm{~V}$ to the left stator comb and a $-8 \mathrm{~V}$ to the right stator comb. A voltage of $10 \sin (\mathrm{Tt})$ was applied to the stage itself. There are two frequencies ranges over which the stage can be driven when the resulting motion can be detected visually under a microscope. The first frequency range is below $1 \mathrm{~Hz}$, where the linear resonator is moving slowly enough that the viewer can see the device cycling back and forth. The second frequency range is around the resonant frequency, which for this device was visually approximated to be $1.85 \mathrm{KHz}$. At resonance, the devices achieve maximum deflection and the linear resonator becomes a blur when viewed under the microscope. While the stage was being driven at resonance, the input fiber was positioned so that the laser spot shown on the edge of the linear stage within the comb split. The detector fiber was then brought in and its position was varied to determine the optimum coupling position. The detector fiber output signal on the oscilloscope was monitored to achieve an optimum voltage difference and optimum output waveform shape. The variation in waveform profiles as a result of alignment will be discusses in a later section.

After actuation the sinusoidal driving signal was shut off. Because the stator voltages were left on, the resonator stage was held in the zero state. After the resonator stopped, the stator voltages were turned off. The probe tip was then introduced onto the right side of the stage so that it could be pushed left. In the earlier experiment there was no reference point so the zero displacement was chosen to be 
the point where the reflective surface was closest to the optical fibers. Now there is a rest state of the MEMS device. So the zero reference point will be chosen at the left side of the resonator stage in the split comb, when the device is at rest. This reference is shown in Figure 4-8. As the stage moves to the left, it will be measured as displacement in the negative direction and as the stage moves right, it will be measured as displacement in the positive direction. As the probe was moved to the left, the distance moved was measured by the linear gauge and the output voltage was recorded from the detector. The signal out of the voltage amplifier which is connected to the detector, was then amplified by the current amplifier. To reduce the amount of noise amplification the lowest amplification setting was used $1 \mathrm{~mA} / \mathrm{V}$. A low pass filter with a cutoff frequency of $30 \mathrm{KHz}$ was also implemented by the current amplifier. The amplified output signal was then input into the oscilloscope. To help further eliminate noise the measured signal was averaged over 128 samples. Since the amount of reflected light that was being coupled into the detector fiber was measured as a DC voltage level, the signal was DC coupled into the oscilloscope.

A graph of the final measurement data is displayed in Figure 4-9. The data shows that as the resonator stage moves to the left the output signal will increase and then decrease. The graph will be compared to the measured electrostatic displacement measurements.

\subsection{Optical Measurement of Static Electrostatic Deflection}

The next experiment is to measure the deflection of the linear resonator using electrostatic deflection. Unfortunately, maintaining alignment is difficult over a large periods of time due to the 


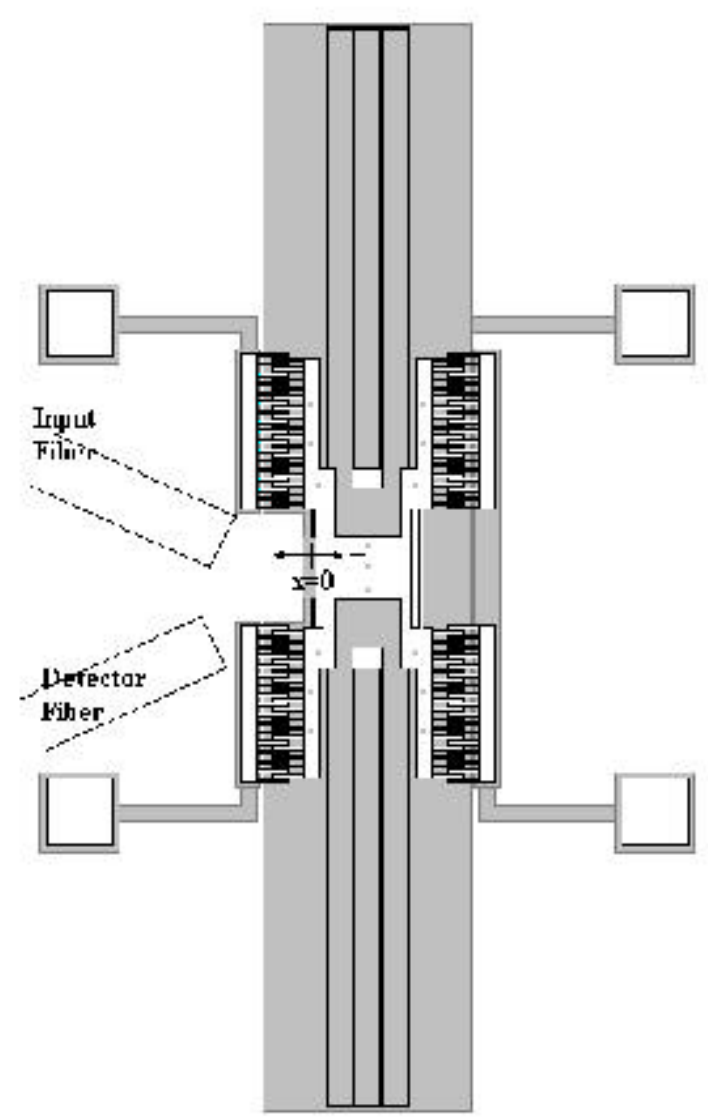

Figure 4-8: Zero Reference of Linear Resonator Position.

mechanical nature of the setup. Therefore this measurement does not use the exact alignment that was used in the forced deflection experiment. The fiber-device configuration used in this experiment is shown in Figure 4-10. Using the same method described earlier, detector fiber alignment was maximized by maximizing the output signal while the device is driven at resonance. This alignment produced a stronger output signal.

The linear resonator stage was deflected by placing a $+8 \mathrm{~V}$ signal on the left stator comb and a $8 \mathrm{~V}$ signal on the right stator comb. A DC voltage signal was then applied to the linear resonator stage. 


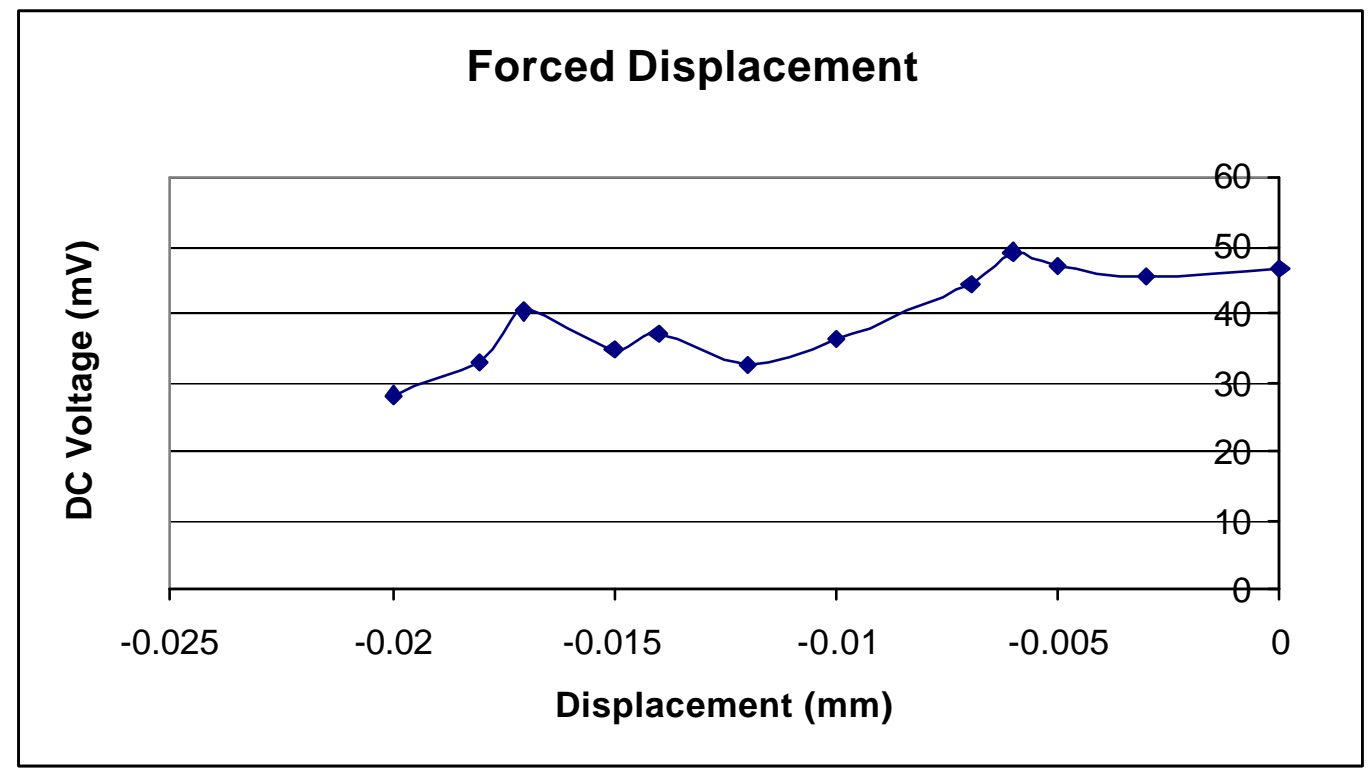

Figure 4-9: Forced Static Displacement Versus the Output Detector Signal Voltage

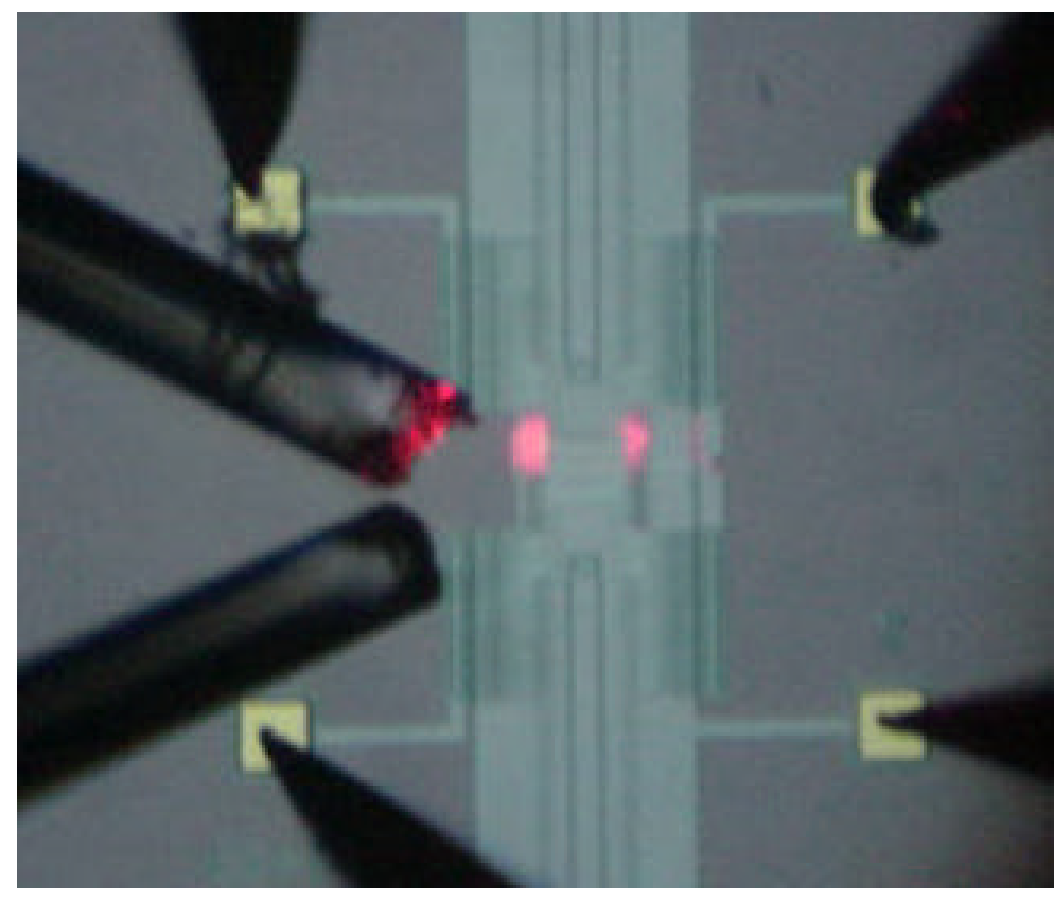

Figure 4-10: Input and Detector Fiber Alignment for Electrostatic Actuated Measurements 
This signal varied from $-10 \mathrm{~V}$ to $+10 \mathrm{~V}$. When the $\mathrm{DC}$ voltage was negative the linear resonator displaced to the left of the zero point, and as the voltage becomes positive the stage displaces to the right.

This time the output detector voltage was recorded as different DC voltage signals were applied to the linear resonator stage. A graph of this data measurement is shown in Figure 4-11. For the negative voltage, which corresponds to a negative displacement, this graph is very similar to the one produced in the forced static displacement. The output voltage increases and then decreases again. Because the device will not achieve the same degree of deflection when electrostatically actuated as when the stage was forced using a probe tip, the graph in Figure 4-12 does not extend to the left to the

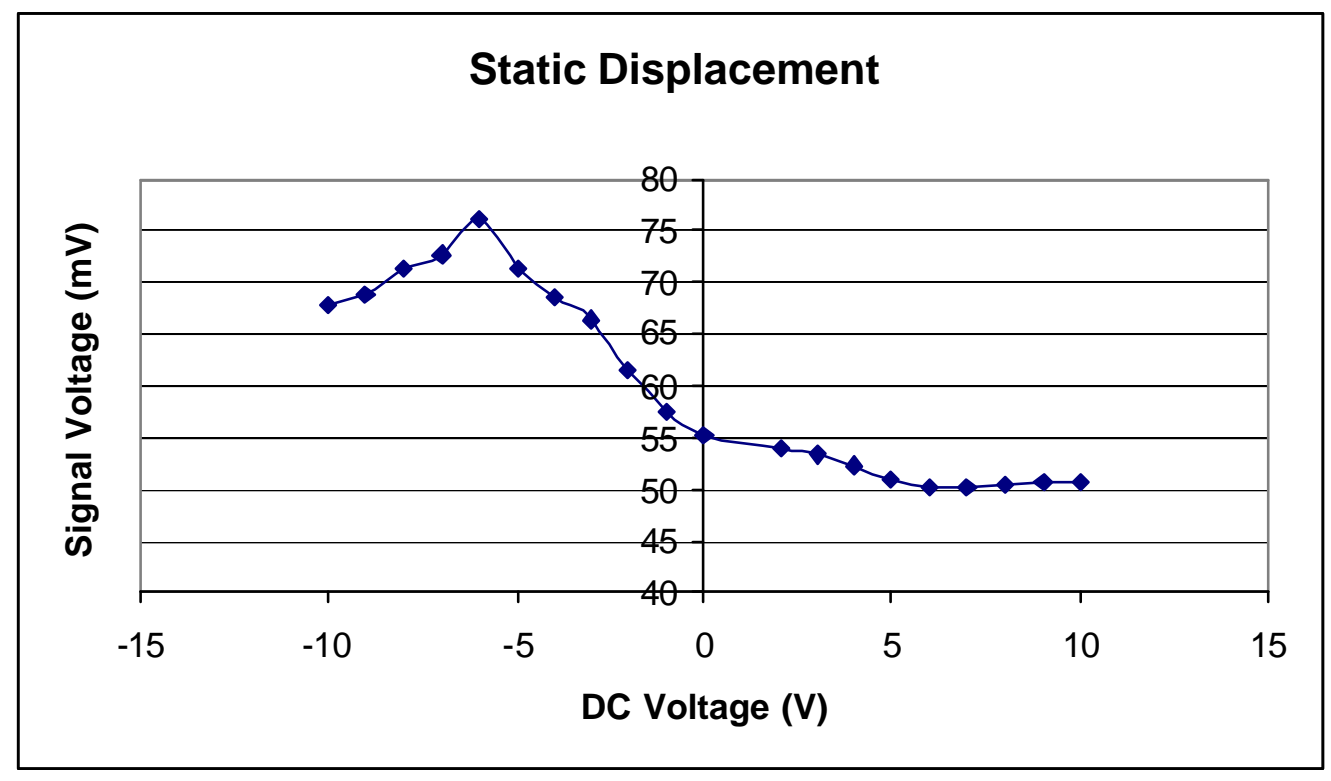

Figure 4-11: Measured Deflection Using DC Electrostatic Displacement of the Linear Resonator Stage 


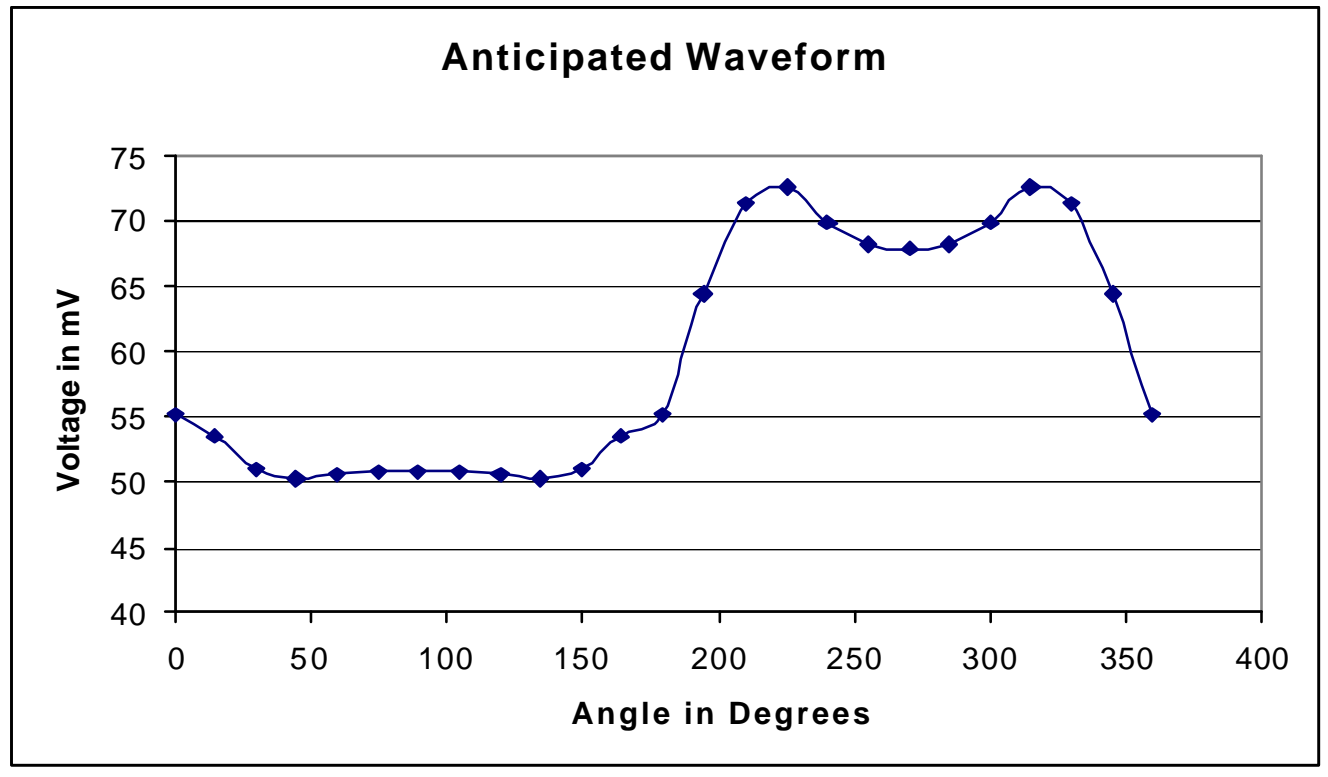

Figure 4-12: Predicted Low Frequency Output Waveform

same extent as the graph in Figure 4-10. Also notice that the output voltage when the stage is at 0V, which is the rest state, is $55.1 \mathrm{mV}$.

Using the data collected to generate the above graph, an anticipated output waveform for low frequency was calculated. The linear resonator is driven by a $10 \sin (? \mathrm{t}) \mathrm{V}$ signal. By calculating the voltage levels at various ? $\mathrm{t}$ values over one period, it was possible to calculate the expected detector voltage for a given value of $\mathrm{Tt}$, using interpolation. The graph of this predicted waveform is shown in Figure 4-12. Because this graph was plotted using static deflection measurements, this predicted waveform is limited to frequencies well below the resonant frequency. 


\subsection{Sinusoidal Actuated Linear Resonator Feedback Signal}

Using the exact same alignment and setup used in the static deflection measurement, the device was actuated with a $10 \sin (\omega \mathrm{t}) \mathrm{V}$ signal. The output signal was DC coupled into the oscilloscope to measure the variations in the level of the output signal. To reduce noise the output signal was averaged over 128 cycles. Measurements were taken at several frequencies: $525 \mathrm{~Hz}$, which is shown in Figure 4-13; $1.5 \mathrm{KHz}$, which is shown in Figure 4-14; $1.575 \mathrm{KHz}$, which is shown in Figure 4-15; $2.05 \mathrm{KHz}$, which is shown in Figure 4-16; and 2.625 KHz, which is shown in Figure 4-17.

In all the oscilloscope graphs, the input voltage signal is a 20VP-P sinusoidal signal, which fluctuates around the zero axis. Because the fiber probe placement, with relation to the voltage bias,

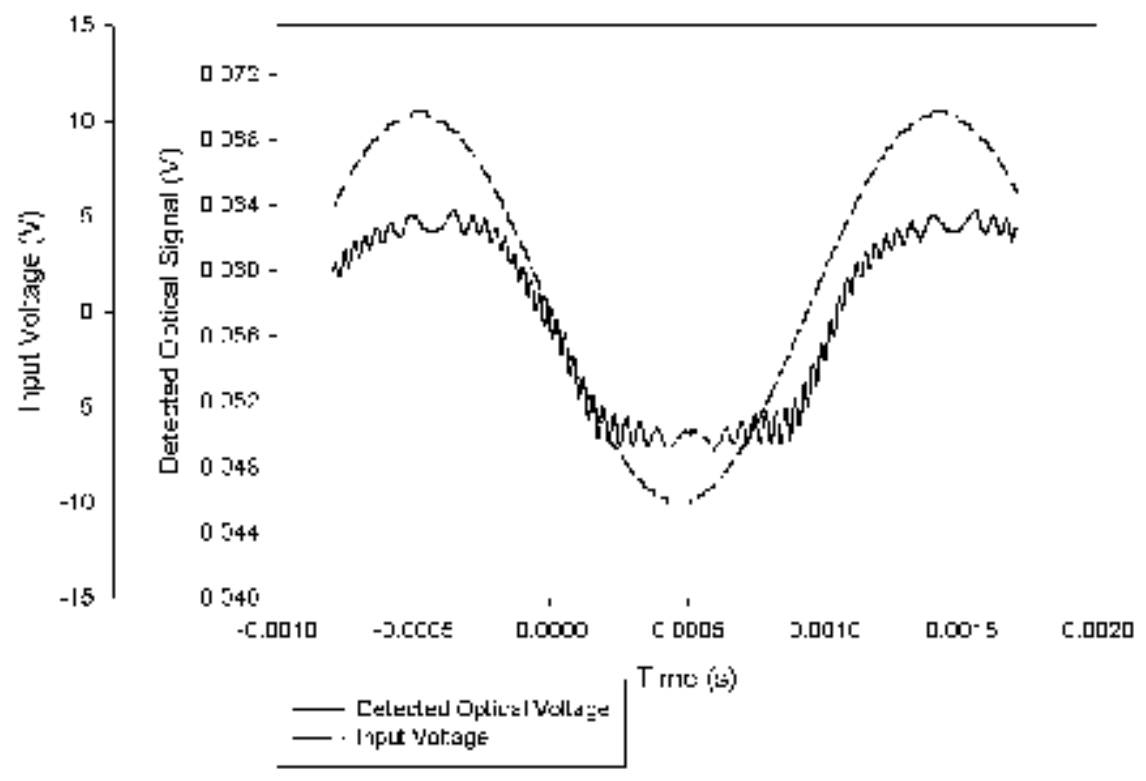

Figure 4-13: 525 KHz Linear Resonator Motion Measurement 


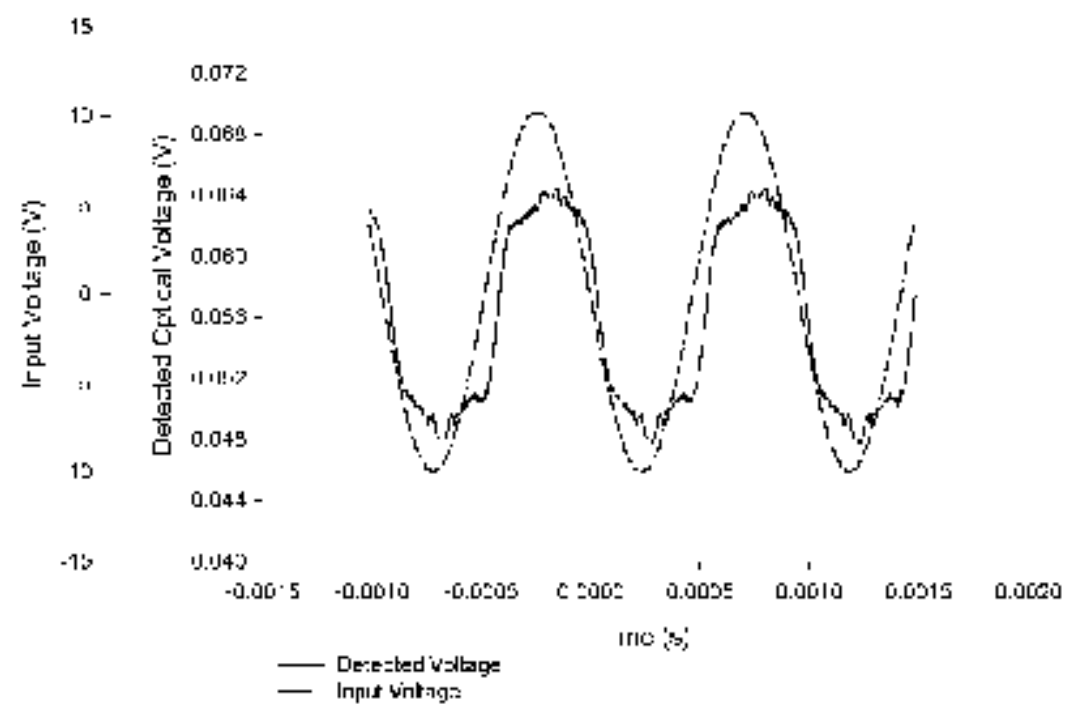

Figure 4-14 1.5 KHz Linear Resonator Motion Measurement

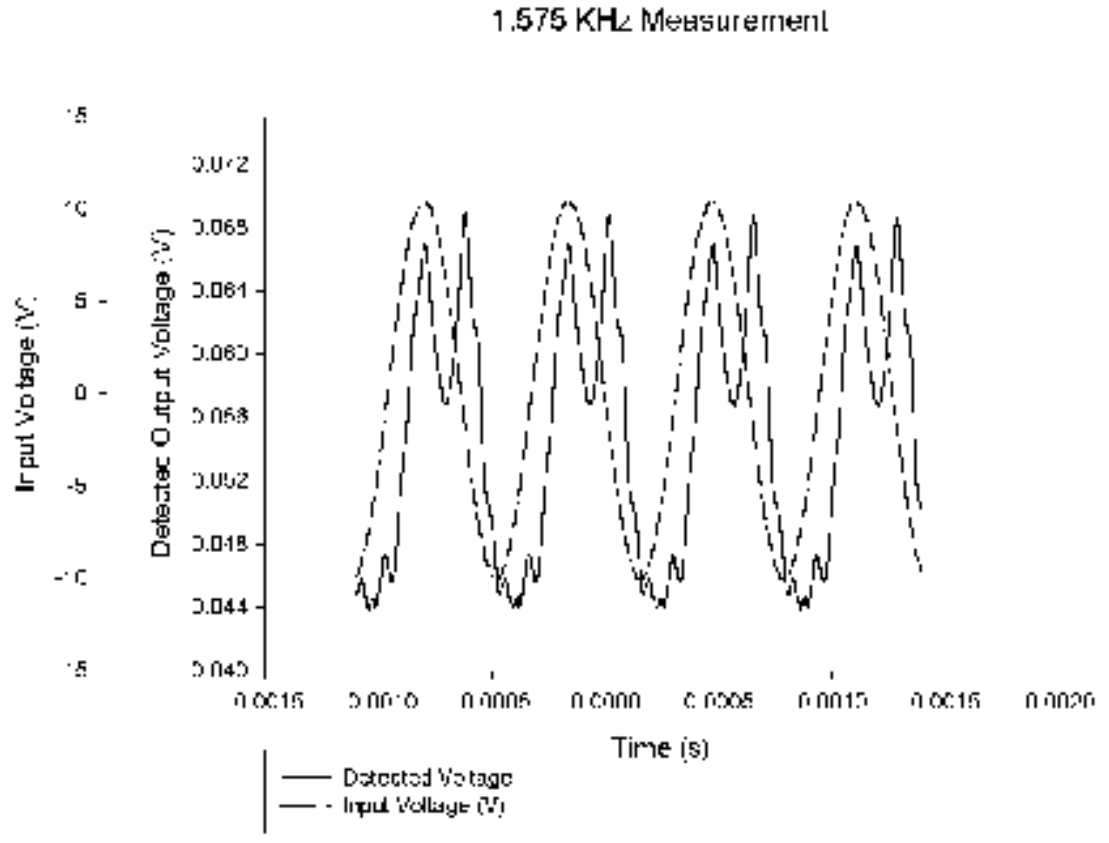

Figure 4-15: $1.575 \mathrm{KHz}$ Linear Resonator Measurement 


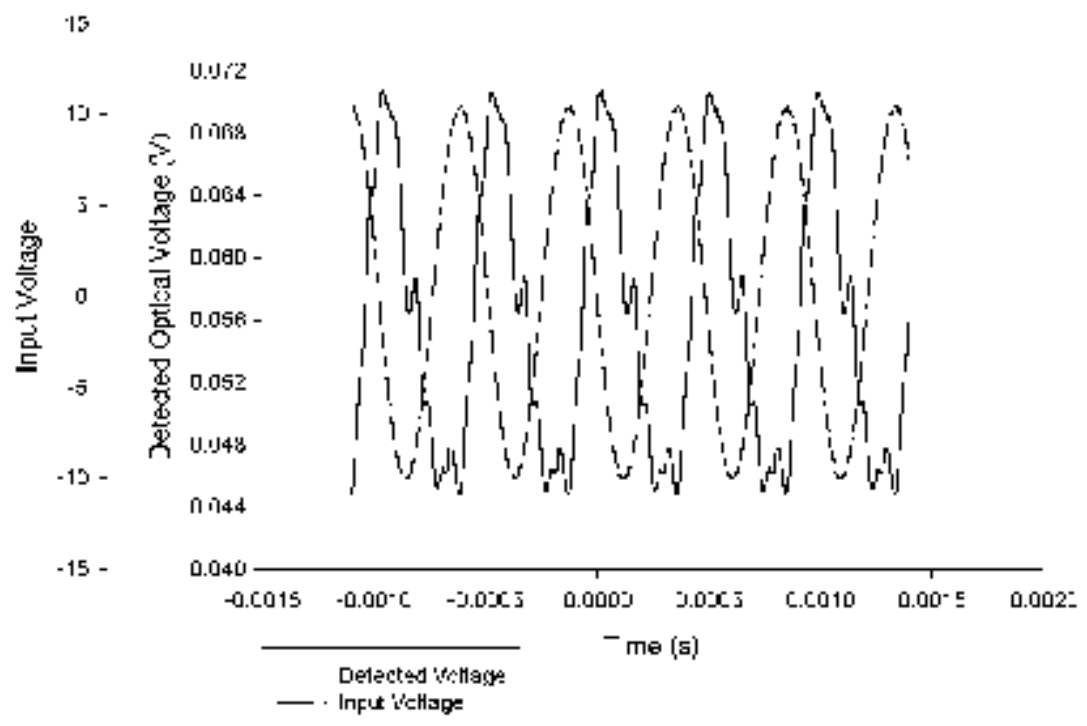

Figure 4-16: $2.05 \mathrm{KHz}$ Linear Resonator Motion Measurement

$2.625 \mathrm{KHz}$ lHejsurement

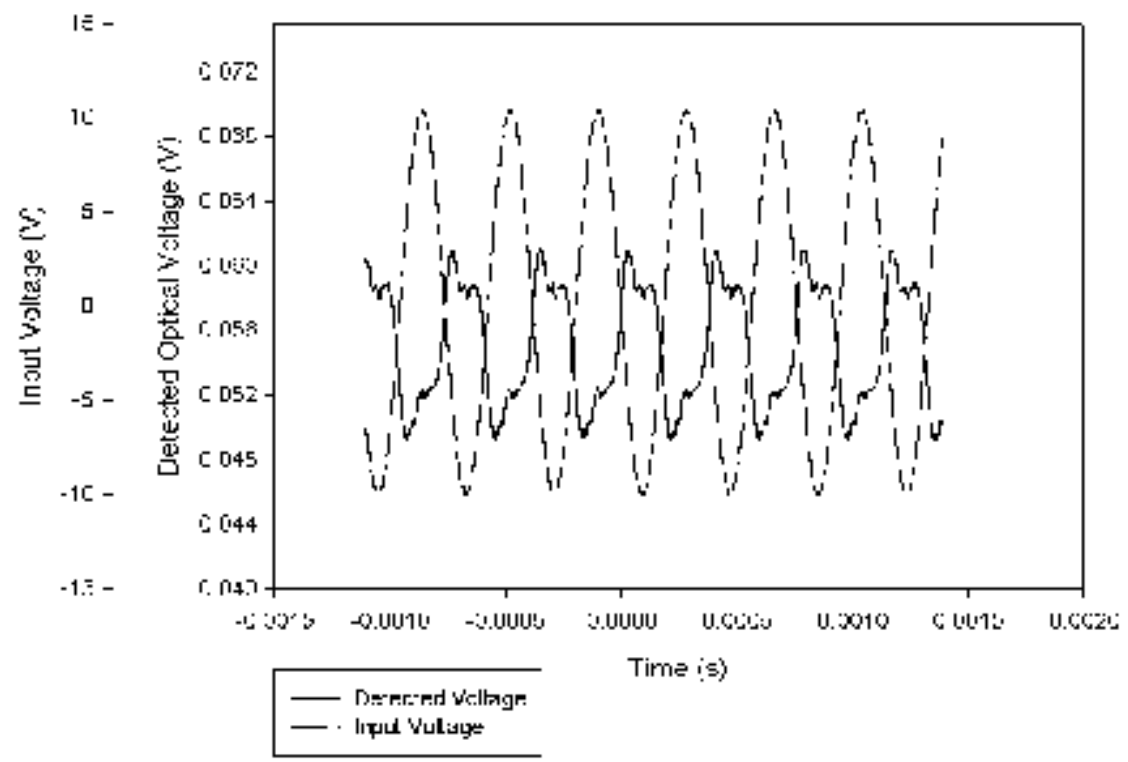

Figure 4-17: $2.625 \mathrm{KHz}$ Linear Resonator Motion Measurement 
created an inverted output signal, compared to the input voltage, the input voltage signal on the graphs has been inverted. The detected signal is DC coupled and the signal fluctuates around a DC offset, of $55.1 \mathrm{mV}$.

Figure 4-13 displays the detected optical signal when the stage was actuated at the lowest frequency, $525 \mathrm{~Hz}$. As one can see on the graph, the output waveform looks almost sinusoidal; it does not take the shape of the anticipated waveform derived in the earlier section. This is because at extremely low frequencies the dominant force acting on the linear resonator is the spring restorative force and the linear resonator stage does not deflect strongly. Therefore the stage never moves far enough left to reach the point at which the optical output decreases and therefore there is not "waveform inversion" in the positive half of the wave. However the overall waveform does behave as expected. When the inverted sinusoidal driving voltage is at its most negative value the linear resonator stage has moved to the farthest right and the output signal should be at its lowest value, since the stage is the farthest it will be from the fibers. Similarly, when the inverted sinusoidal, driving voltage is at its most positive value the linear resonator stage has moved to the farthest left and the output signal should be at its highest value, since the stage is the closest it will be from the fibers. Notice that at this very low frequency the output signal is almost in-phase with the input signal.

Figure 4-14 is a graph of the detected optical signal when the driving frequency for the linear resonator device is $1.5 \mathrm{KHz}$. Once again the output waveform looks more sinusoidal than the anticipated waveform shown in Figure 4-12. At this frequency the spring restorative force is still dominating the linear resonator motion. On the graph the peak-to-peak value is larger than that for the $525 \mathrm{KHz}$ signal. The $1.5 \mathrm{KHz}$ signal is also showing a phase shift which is approaching 45E. Both the 
increase in signal fluctuation and the phase shift are expected as the frequency approaches the resonant frequency of $1.85 \mathrm{KHz}$.

Figure 4-15 shows the output waveform at a frequency of $1.575 \mathrm{KHz}$. In this picture the output waveform takes on a profile that is similar to the anticipated waveform. The peak-to-peak value of the wave continues to increase and the phase shift keeps approaching 90E Figure 4-16 shows the output signal at a frequency of $2.05 \mathrm{KHz}$, which is beyond the calculated resonant frequency. In this graph the waveform no longer takes the profile of the anticipated waveform, because the device has already reached and passed resonance. Peak-to-peak voltage is very large at this point because the device is still near resonance and the phase shift has exceeded 90E. The final graph, Figure 4-17, shows the output signal when the resonator is driven at $2.625 \mathrm{KHz}$. Here the peak-to-peak value of the output waveform has reduced greatly, because the resonator stage is being driven too fast to achieve full deflection. The phase shift of this waveform is approaching $180 \mathrm{E}$, as theoretically expected.

\subsection{Resonant Frequency Measurement}

Given that the stage displacement and resulting magnitude of measured optical detection voltage variation reaches maximum at resonance, the fiber optic probes can be used to experimentally detected the resonant frequency. The fiber probe alignment used in the previous two measurements was able to be maintained long enough to make a resonant frequency measurement. The only change that was made from the above measurement setup is that the coupling of the detector signal to the oscilloscope was changed to AC from the previous DC coupling. Changing the oscilloscope coupling 
to $\mathrm{AC}$ would remove the $\mathrm{DC}$ offset value and make the peak-to-peak voltage measurements easier. As the linear resonator was stepped through several frequencies, the peak-to-peak amplitude voltage of the output signal was measured. A graph displaying the resulting voltages is shown in Figure 4-18. On this graph of the data the peak measured voltage occurs at $1.85 \mathrm{KHz}$, which is the observed resonant frequency. In chapter 3 the theoretical frequency was calculated to be $1.89 \mathrm{KHz}$. The percent error between the measured resonant frequency and the calculated resonant frequency is $2 \%$.

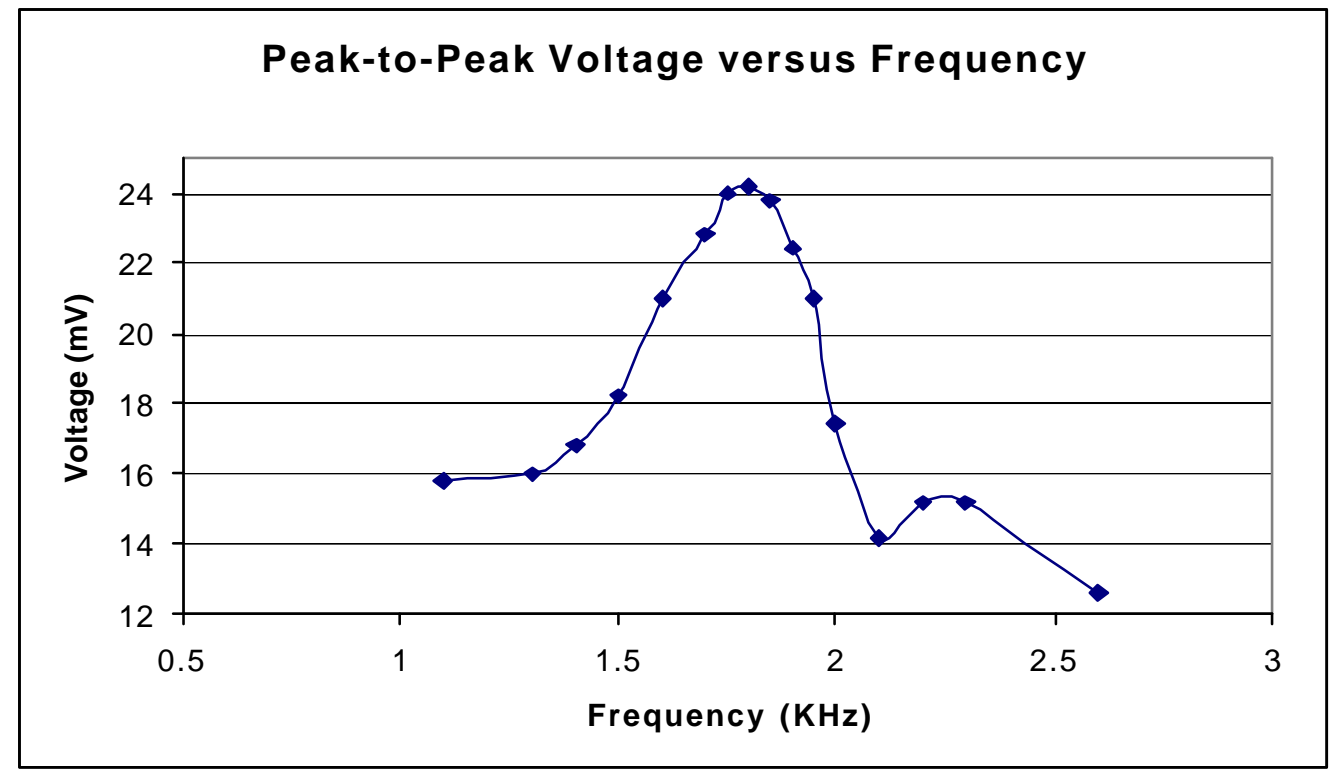

Figure 4-18: Resonant Frequency Measurement 


\subsection{Fiber Alignment Position Relation to Output Waveform}

The position of the input fiber and detector fiber can greatly influence the output voltage signal, both in magnitude and in waveform profile. Maintaining alignment of the optical fibers to the MEMS device over periods longer than a couple of hours was very difficult. Even though the entire probe station setup was isolated on an air table, building vibrations and strains on the fibers would cause the alignment to drift. To demonstrate how the waveform changes with fiber alignment, the linear resonator device was measured using different optical fiber alignments with the same signals that were used previously; $+8 \mathrm{~V}$ on the left comb stator, $-8 \mathrm{~V}$ on the right comb stator and $10 \sin (\omega \mathrm{t})$, where theoperating frequency remained at $2.2 \mathrm{KHz}$. The following figures will show a picture of the alignment of the optical fibers and the resulting output waveform.
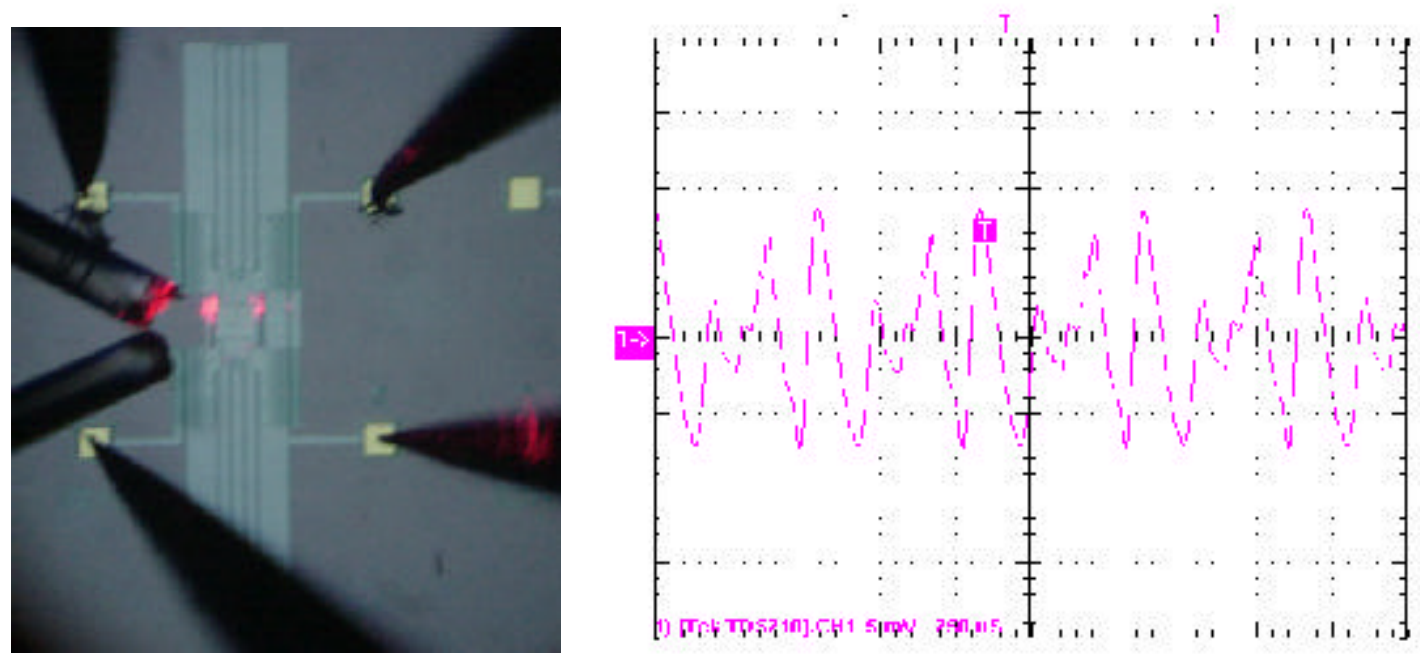

Figure 4-19: Multimode Input Fiber Alignment1 and the Oscilloscope Curve Trace of the Resulting Output Signal (x-axis division is $250 \mu \mathrm{S}$ and y-axis division is $5 \mathrm{mV}$ ). 

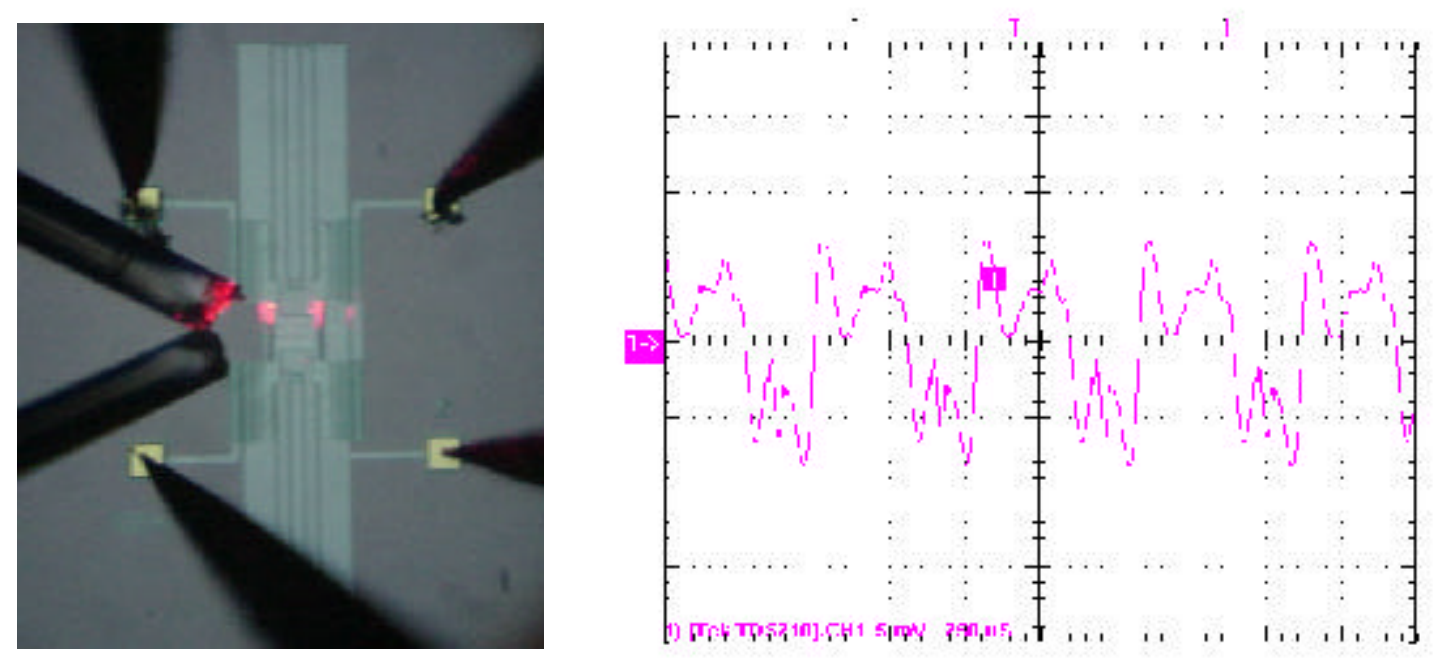

Figure 4-20: Multimode Input Fiber Alignment 2 and the Oscilloscope Curve Trace of the Resulting Output Signal (x-axis division is $250 \mu \mathrm{S}$ and $\mathrm{y}$-axis division is $5 \mathrm{mV}$ ).
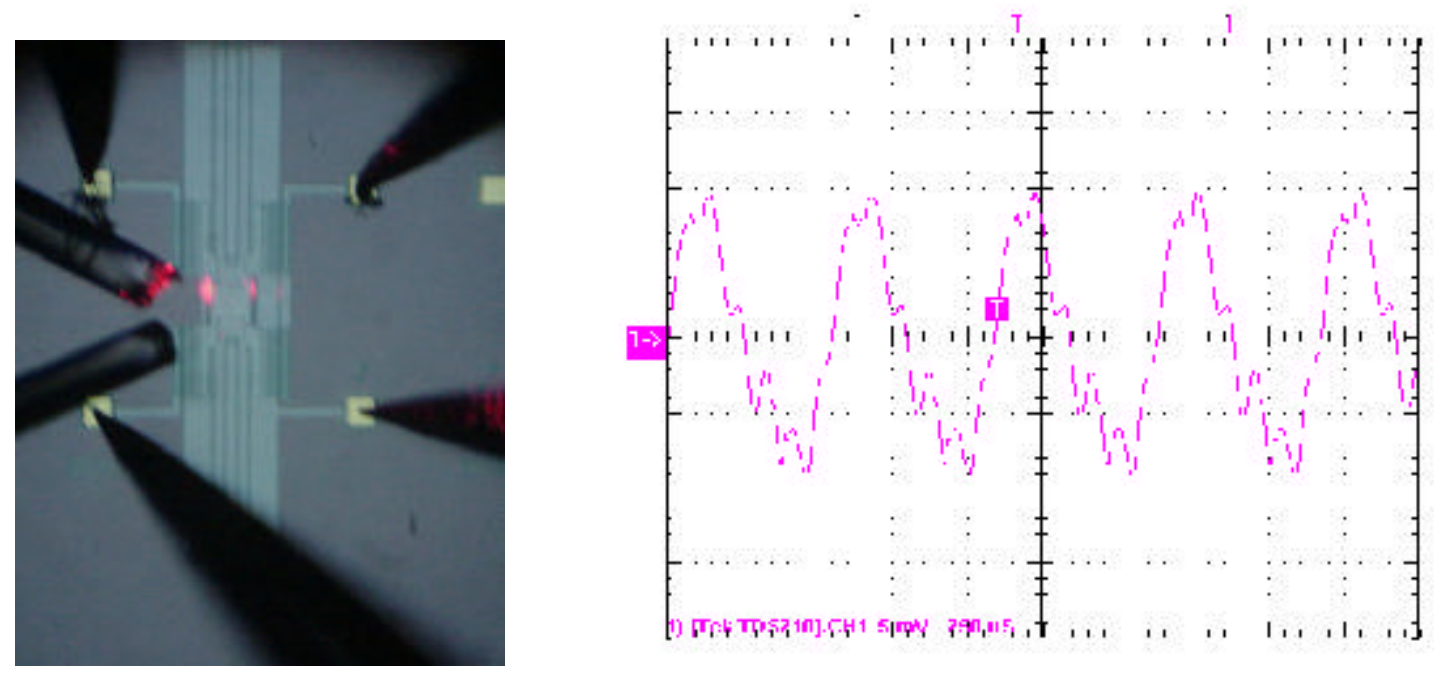

Figure 4-21: Multimode Input Fiber Alignment 3 and the Oscilloscope Curve Trace of the Resulting Output Signal (x-axis division is $250 \mu \mathrm{S}$ and $\mathrm{y}$-axis division is $5 \mathrm{mV}$ ). 

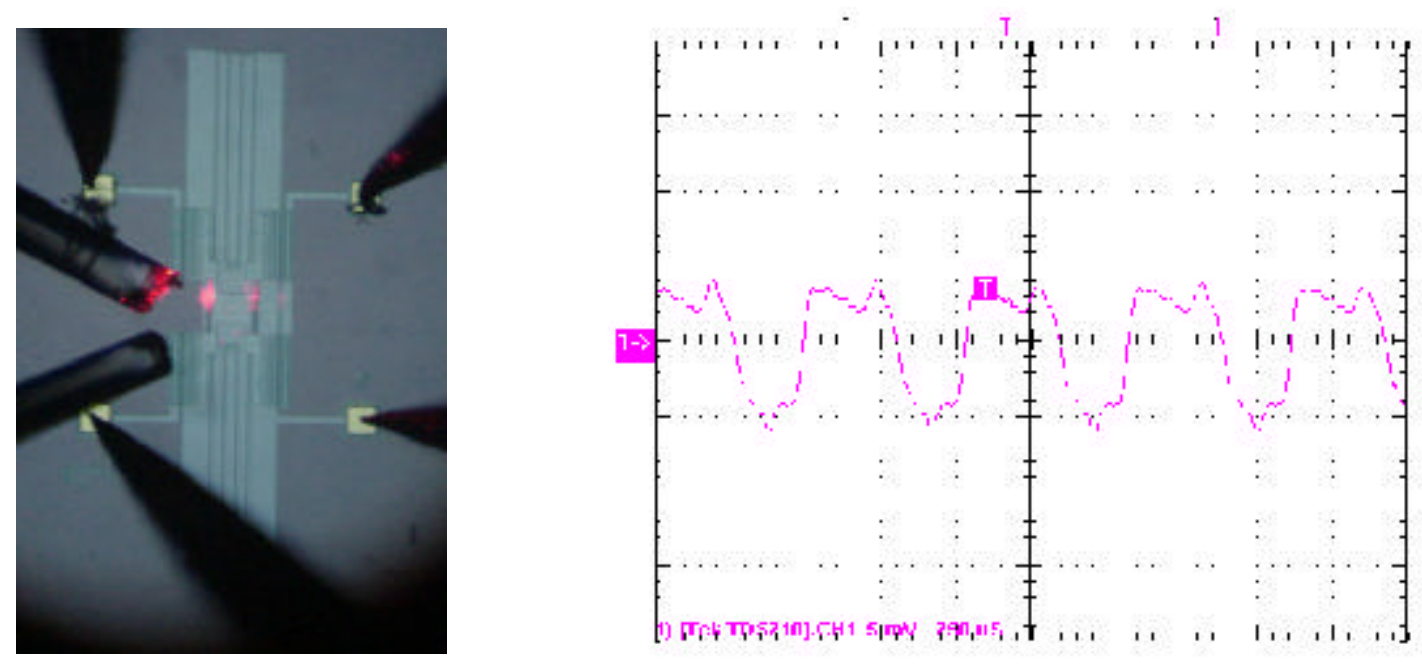

Figure 4-22: Multimode Input Fiber Alignment 4 and the Oscilloscope Curve Trace of the Resulting Output Signal ( $\mathrm{x}$-axis division is $250 \mu \mathrm{S}$ and y-axis division is $5 \mathrm{mV}$ ).

\subsection{Single Mode Fiber Measurements}

All the previous fiber probe experiments utilized multimode fibers for both the input and the detector fiber. The advantage of a multimode input fiber is that it will be easier to couple to, it can carry more optical power, and it can produce a brighter and larger optical probe spot size for the input upon the linear resonator stage edge. Since interference effects between the light introduced by a single mode fiber and the light reflected from the stage will be a result of displacement and not a result of modes, the above experiments are repeated using a single mode fiber to observe the measurement resolution using interference. The smallest optical fiber available in the lab had a core diameter of approximately $8 \mathrm{Fm}$, which is typical for single mode fibers. However, these experiments use a Helium Neon laser which has a wavelength of $632.8 \mathrm{~nm}$, and the single mode fiber was designed to carry 
wavelengths of 1.3 or $1.5 \mathrm{Fm}$. Therefore this fiber is not truly single mode, but it carries three to four modes of each polarization.

Using this fiber a set of measurements was undertaken similar to the ones taken using the multimode input fiber. However, because the fiber core is smaller, the intensity of light being emitted by the input fiber was greatly reduced, and the spot size was smaller creating more difficulties in positioning and maintaining fiber alignment.

\subsection{Displacement Measurements Using a Single Mode Input Fiber}

Once again, before optically probing the linear resonator, to establish how optical measurement of displacement could be made, a displacement measurement was made using a single mode input fiber, a multimode output fiber, and a large reflecting surface. The same setup was used as in Section 4.2. A single mode fiber was used as the input fiber, so that the optical power source would be coherent, and the measurements could determine the effectiveness of measuring displacement using interference effects. A multimode fiber was chosen as the output fiber because the multimode fiber has a large acceptance angle, and would be easier to align. The multimode fiber would carry more optical power, since the input optical power was expected to be small. The input and detector fibers were attached to probe manipulators so that they could be aligned under the probe station microscope. The blunt end of a probe holder was used as a large reflecting surface.

The coupling of laser light into the single mode fiber is very critical to achieve a maximum input signal for the optical fiber probe. To maximize the coupling into the single mode fiber, first the position of the fiber end face was manipulated while the output of the fiber was examined visually in reflection. 
Then, using the probe manipulators, the input fiber and the detector fiber were aligned so that the two fibers were butt-coupled. A chopper was used to interrupt the laser light, and the output of the voltage amplifier was attached to an oscilloscope. By maximizing the output square wave on the oscilloscope, the alignment of the two fibers was maximized and then the coupling of laser light into the input fiber was re-tuned.

After achieving maximum coupling, the fibers and the reflecting surface were aligned in the probe station. The output of the voltage amplifier, which amplifies the output of the optical detector, was connected to a voltmeter. The digital linear gauge was positioned against the probe manipulator holding the reflecting surface to measure the distance displaced. Once again the zero point was take to be when the reflecting surface was at the closest point to the optical fibers. The measured signal is shown in Figure 4-23.

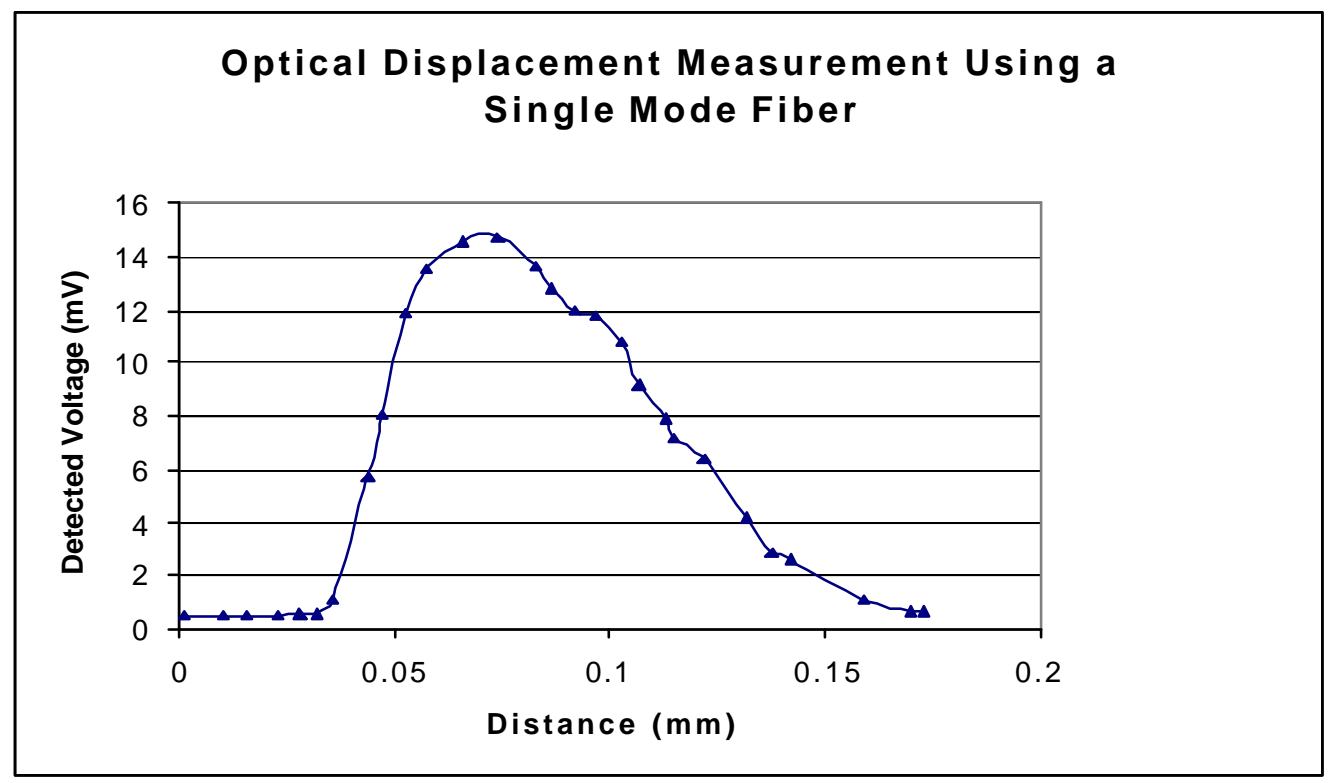

Figure 4-23: Measured Optical Displacement of a Reflecting Surface Using a Single Mode Input Fiber and Multimode Detector Fiber 
This displacement measurement takes a similar form to that in Figure 4-4, when a multimode fiber was used as an input fiber. The difference between the two graphs is that the single mode graph displays a smoother curve, which is expected since multimode fibers produce speckle noise. The single mode fiber also dies off more quickly and measures a shorter range of distance. This is because the single mode fiber has a smaller core and smaller output divergence angle than the multimode fiber, and the detector probe is therefore out of range more rapidly than with the multimode fiber.

\subsection{Sinusoidal Actuated Linear Resonator Feedback Signal Using a Single Mode Input Fiber}

Using the single mode input fiber, a repeat of the types of measurements taken earlier: forced static displacement, electrostatic displacement, actuation measurement, and resonant frequency measurement, were to be taken. The first step to taking these measurements was to align the input and detector fibers to the linear resonator. In the single mode fiber experiments a different comb driven, linear resonator was used. This device also had $550 \mathrm{Fm}$ flexures, but it did not have the fabricated superstructures which were added to increase the vertical height of the resonator stage edge. A new structure was used for measurements because the original MEMS device was shorted out. MEMS are driven with large voltages which generates a high electrostatic field around the devices and dust particles become attracted to the structures. A dust particle which landed on one of the stator comb drives in the linear resonator used in the multimode measurements and permanently shorted out the resonator device. 
The reflectance surface of the resonator stage edge was reduced in these measurements, and other factors limited the intensity of the feedback signal. The primary two were the lower intensity light incident on the resonator edge and the small spot size divergence. These factors made alignment of the optical fiber probes very difficult.

The optical fibers were aligned with the linear resonator in the same fashion as described in previous sections. Visually the resonant frequency of this device was also $1.85 \mathrm{KHz}$. While operating at resonance, the fibers were aligned to the resonator so that the detector received a signal. The position of these fibers is shown in Figure 4-24. As expected the signal was weak and the current amplification was set for $50 \mathrm{FA} / \mathrm{V}$, which was a very large amplification, that also amplified noise in the system.

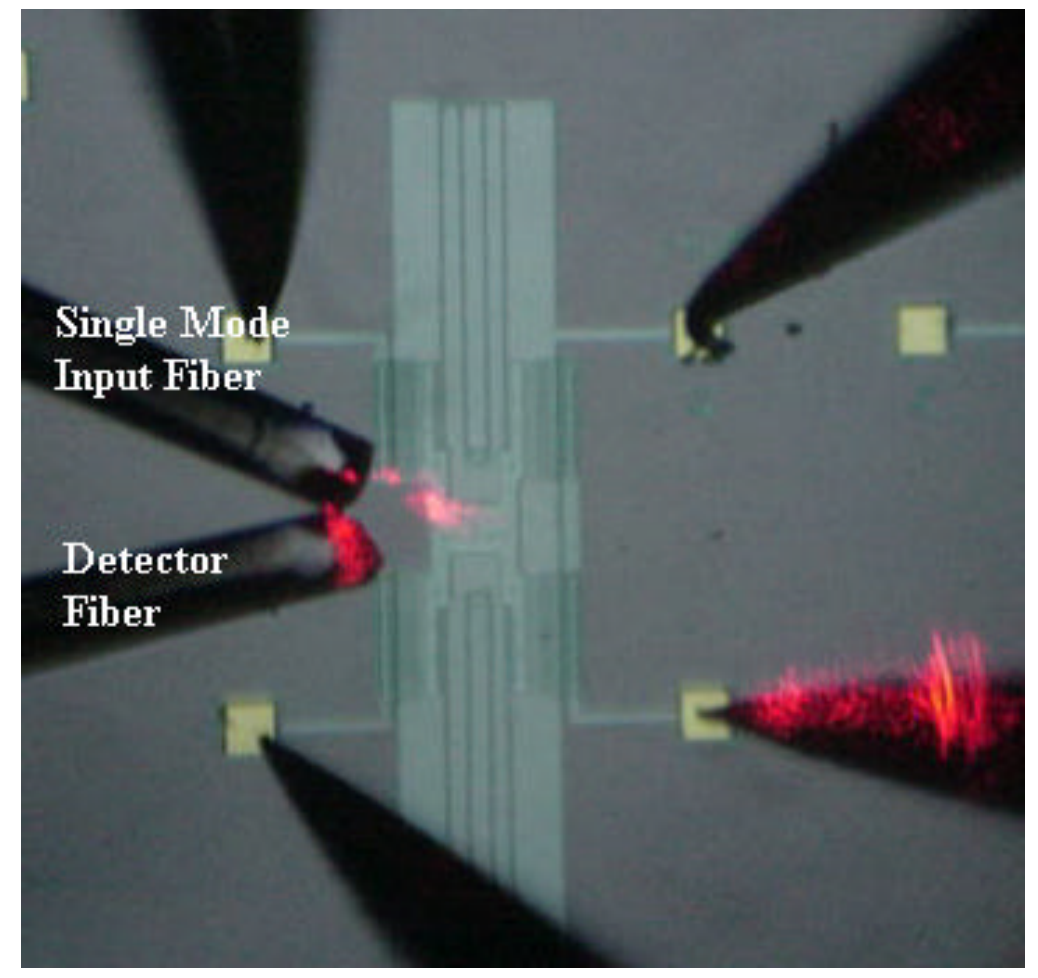

Figure 4-24: Single Mode Fiber Alignment 
The output waveform, which was displayed on the oscilloscope, varied in voltage from 182 $\mathrm{mV}$ to $186 \mathrm{mV}$, which was a measurement of signal intensity variation as the resonator stage oscillated over a distance of less than $40 \mathrm{Fm}$. Detecting the fine voltage variations in the signal for a resonator stage displacement of $1 \mathrm{Fm}$ was very difficult. A low frequency signal variance was superimposed upon the output signal creating a signal drift, which was on the order of 4 or $5 \mathrm{mV}$. This drift compounded the difficulties in taking measurements. Any collected voltage had a margin of error of \pm $2 \mathrm{mV}$.

Neither the forced static displacement or electrostatic displacement measurements could be made with any confidence in the results. While not able to take quantitative measurements with known voltages, the detected signal did demonstrate an output signal which varied with frequency. The following figures display the output signal at different frequencies. Figure 4-25 shows the output waveform while the resonator is being driven at $462 \mathrm{~Hz}$. Figure 4-26 shows the output waveform while the resonator is being driven at $925 \mathrm{~Hz}$. Figure 4-27 shows the output waveform while the resonator is being driven at $1.387 \mathrm{KHz}$. Figure 4-28 shows the output waveform while the resonator is being driven at $1.85 \mathrm{KHz}$. Figure 4-30 shows the output waveform while the resonator is being driven at 2.312 KHz.

In Figure 4-25, when the frequency is the lowest, the output waveform is almost in perfect synchrony with the input. As the frequency increases the phase shift increases until Figure 4-28. In this figure the frequency is at resonance $(1.85 \mathrm{KHz})$, and the phase shift appears to be $90 \mathrm{E}$. In Figure 429 , the frequency is above resonance and the phase shift is approaching $180 \mathrm{E}$. So the detected signal is performing as expected. 
$462 \mathrm{H}_{4}$ Measulermerll

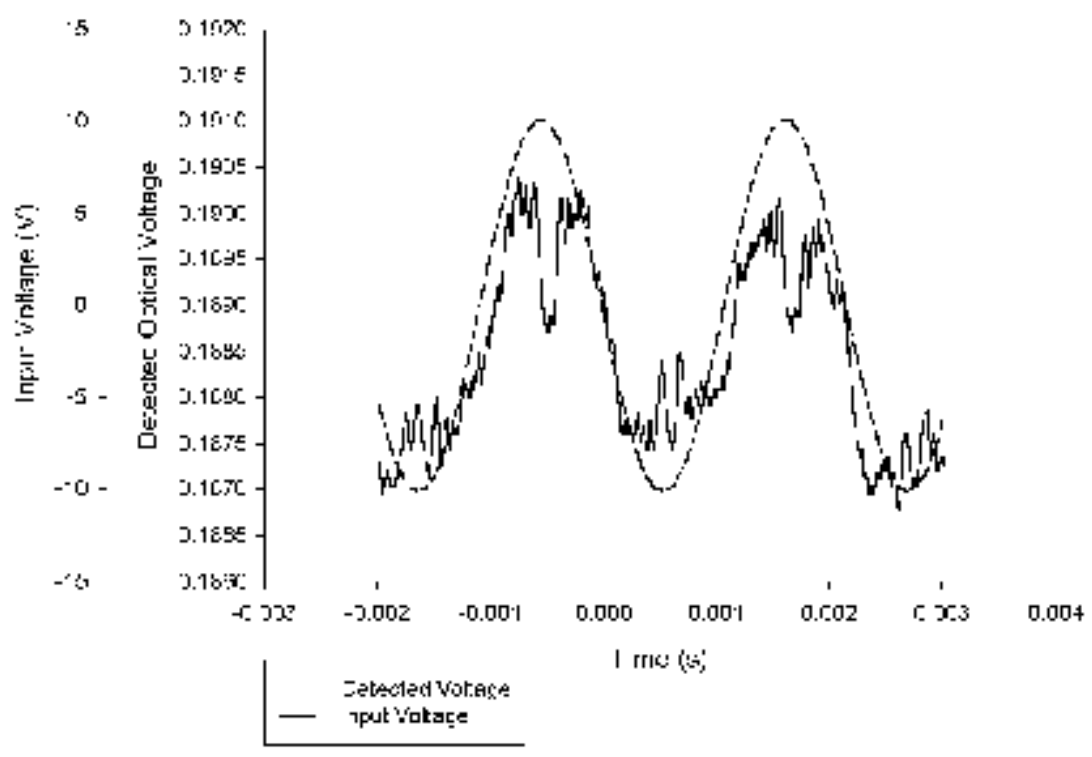

Figure 4-25: 462Hz Linear Resonator Motion Measurement

$925 \mathrm{~Hz}$ Mleasurement

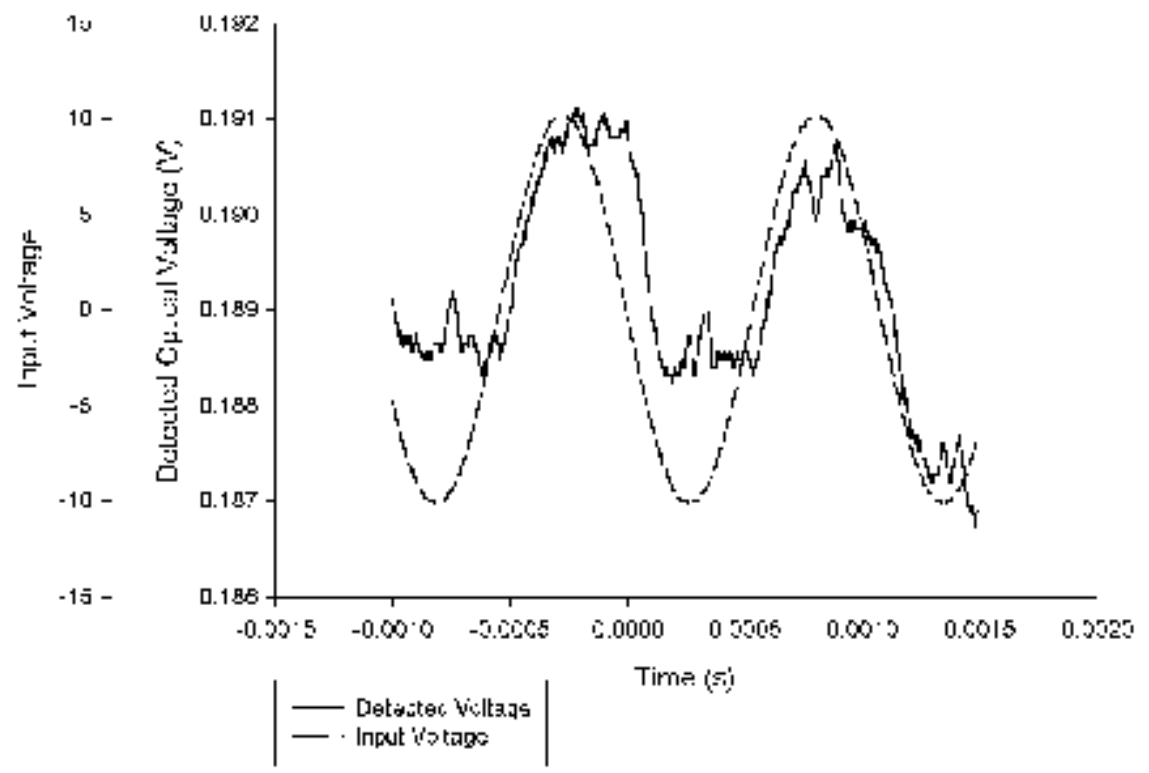

Figure 4-26: 925Hz Linear Resonator Motion Measurement 


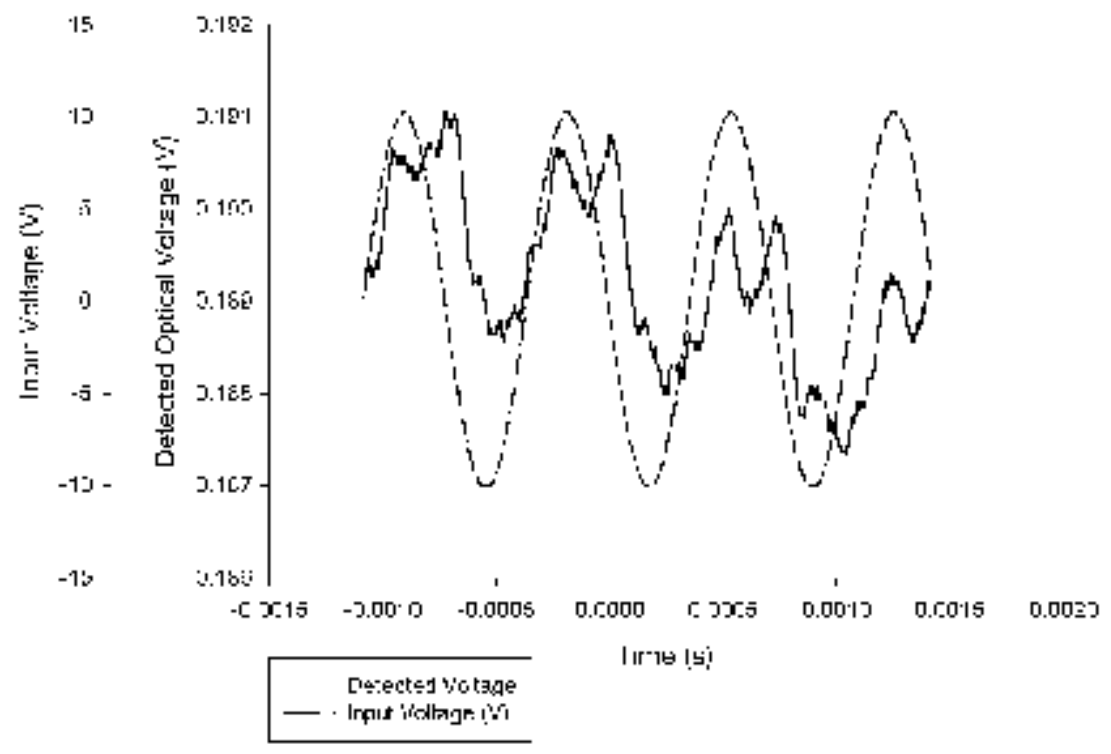

Figure 4-27: 1.387KHz Linear Resonator Motion Measurement

\section{$1.85 \mathrm{KHz}$ hHeasurement}

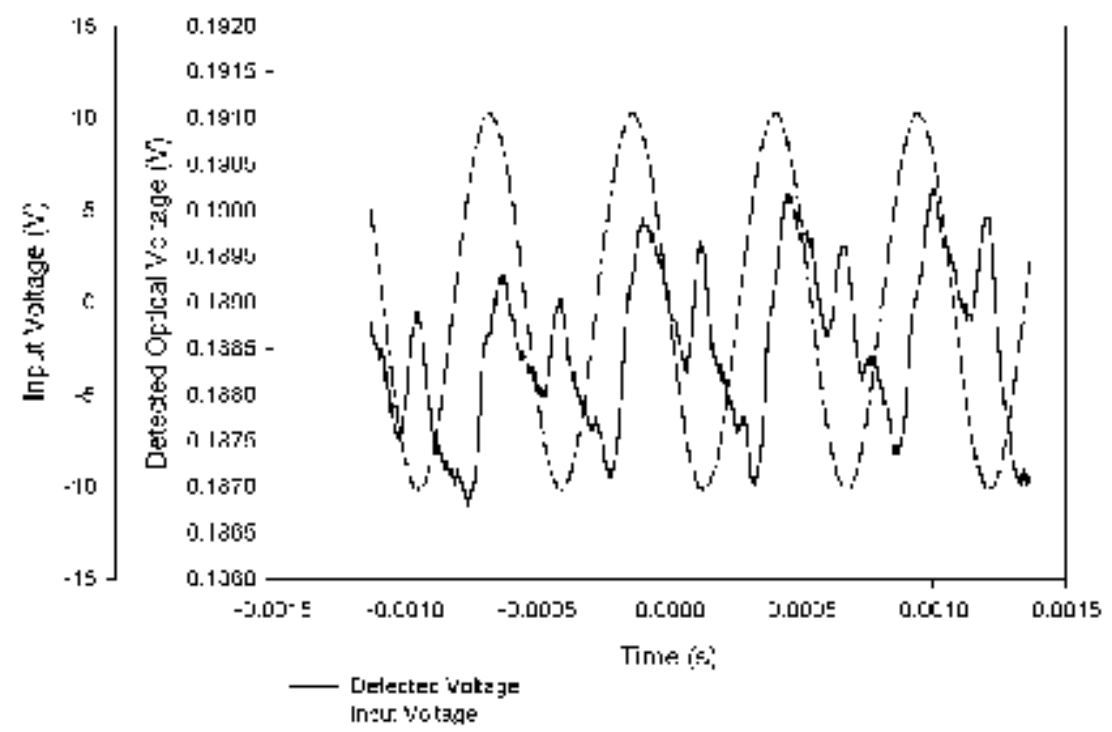

Figure 4-28: 1.85KHz Linear Resonator Motion Measurement 


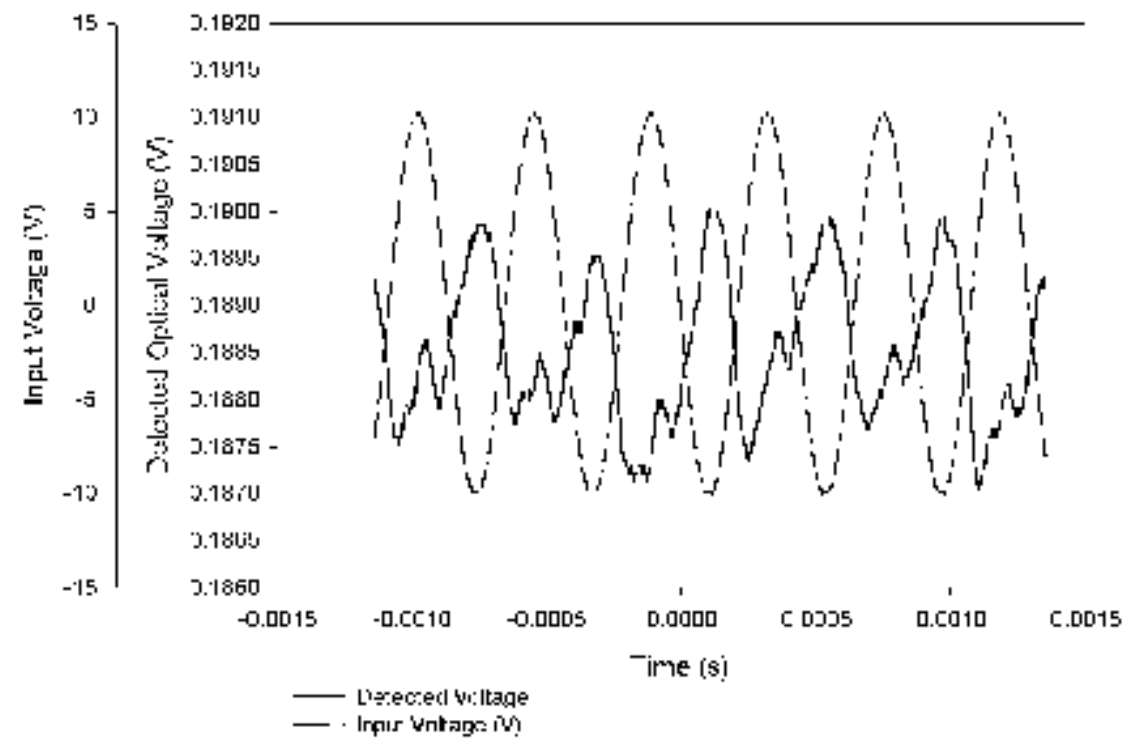

Figure 4-29: $2.312 \mathrm{KHz}$ Linear Resonator Motion Measurement

\subsection{Multimode and Single Mode Measurements}

The objective of these experiments were to demonstrate an optical technique that can be used to characterize a linear comb resonator device. Two different optical phenomenon can detect the displacement of the resonator: optical divergence and interference. To demonstrate the effectiveness of these techniques, two different input, optical fibers were used. A multimode input fiber carries multiple number of modes, and therefore the emitted light is a conglomeration of different optical wave fronts. Multimode optical measurements measure the changes in optical output intensity, which varies with the changes in the optical path length. As demonstrated in this chapter, the multimode input fiber can detect 
the motion of the resonator stage, and can be used to measure operating frequency, phase shift and resonance.

The greatest advantage of using a multimode optical fiber to illuminate the stage edge is that the fiber can carry more optical power, and therefore increase the intensity of the input signal. Larger input fibers also have the advantage of a larger emitted spot size, which relaxed the alignment requirements of the optical probe system.

Using a single mode input fiber is required for measuring the effectiveness of interference in detecting stage motion. Single mode fibers carry only the primary mode, and therefore emit light which has a unified field, which is required for interference. Intereference measurements have the potential to increase the displacement measurement resolution. However, the single mode input fiber also has certain disadvantages. Because the fiber carries only the primary mode, the amount of optical power carried by the fiber is low. In this chapter the decrease of the input optical signal required a large amplification of the output signal, which also amplified noise in the system and increased the margin of measurement error. The single mode fiber also emitted a smaller spot size which increased the difficulty of the detector fiber alignment. However, the experiment demonstrated that a single mode input fiber can be used to detect resonator motion, and it was able to successfully measure the phase shift in the output signal.

The limitation in the single mode fiber measurements was created by limitations with the available facilities in the labs at WVU. To take an accurate displacement measurement using a single mode input fiber some different steps could taken to increase the signal intensity. One detriment to the experiments in the lab is that the fibers are only cleaved and not polished. Polishing the fibers would 
make the end faces smooth and there would be lower loss. Smooth end faces would also eliminate any odd beam deflections. A second alternative is to use a laser with greater optical power. While a HeNe laser was used to allow for visual alignment, the use of an infrared laser should also be explored, so that the measurements could be truly single mode.

Measurement of the detected optical signal required a voltage and a current amplification of the electrical signal. A more sensitive optical detector, and less electrical amplification can also reduce the introduction of noise in the system. 


\section{Chapter 5}

\section{Integrated Optical Metrology}

\subsection{Bulk Optical Metrology Review}

In the previous chapter, it was demonstrated that optical waveguides, in the form of optical fibers, can be used to characterize a comb driven, linear resonator MEMS device. Using multimode and single mode fibers, motion detection is possible to measure specific device operation characteristic like resonant frequency, phase shift and deflection. This optical fiber measurement technique, a form of bulk optical metrology (BOM) has been used to characterize various MEMS structures in laboratory settings. While effective, the use of the optical fiber probes have certain limitations.

BOM is a method which can accurately test and characterize the behavior of many types of devices, however, the application of this measurement method is limited to operational settings where the device is accessible to external optical fiber probes. BOM can not be used for monitoring device performance during embedded operation. If operating as an actuator or sensor, the device will be interacting with its environment, therefore in order to use optical waveguides to monitor the device, a less intrusive, integrated form of optical probe must be developed. A second limitation to bulk optical fibers is that the waveguide and micro-optical packaging and alignment system is massive when 
compared to the device being measured. One of MEMS technology's most attractive features is that very complex MEMS devices can be fabricated, which miniaturizes very bulky systems. Even with a developed optical fiber probe system, it would be very difficult to monitor hundreds of devices on a single chip due to the large diameters of the optical fibers [29, 30].

\subsection{Integrating Optical Waveguides with MEMS Devices}

Integrated optics has become a very well established technology with the advancement of fiber optic communication applications and the integration of fiber coupling devices. When discussing integrated optical metrology (IOM) of MEMS devices, several configurations of optical signal probes can be designed depending on what type of integrated optics is being used. There are two basic classifications of integrated optics: planar and non-planar. Planar integrated optics commonly refers to the creation of integrated waveguides on a substrate. Using planar optics for IOM would consist of fabricating optical waveguides next to the MEMS devices and performing measurements similar to those discussed in Chapter 4.

Non-planar refers to the integration of optic devices where the optical axis is not parallel with the surface of the substrate. These methods normally utilize through wafer measurements or surface reflection to detect motion. Optical probing systems can also be created using both planar and nonplanar optical paths. One example application uses optical waveguides, which are fabricated on a separate wafer plane and are positioned above the plane of the resonator stage, to carry optical power to the device. The optical power is then coupled out of the waveguide using gratings or Fresnel lenses at the waveguide ends. This optical beam is transmitted through the wafer where it is coupled back into an 
output waveguide beneath the MEMS die, fabricated on another seperate wafer. Optical measurement of motion is made as the linear stage interrupts the optical path as it resonates. Variations of the setup can be created by using repositioning the output and input waveguides to detect reflected signals. This research path, using hybrid, integrated optics for IOM, is currently being explored by others [33].

An appealing approach to address the limitations of BOM is to integrate the optical probe system. This integrated optical measurement method will potentially be able to monitor the operation of a multiple number of devices throughout their lifetime, and provide an optical measurement probe that is small in size, non-interfering with the electrical and mechanical operation, and permanently aligned optically to the MEMS device [30].

The focus of the remainder of this research dissertation explores the feasibility of using integrated waveguides to perform IOM on the linear resonator device previously characterized with BOM. An integrated version of the Bulk Optical Metrology system described in the previous chapter will have four basic components. One, there must be an optical source, two, a guided optical path, which carries the optical signal to the device being measured, three, a guided optical path, which carries the reflected signal away from the device, and four, a detector, which converts the optical signal to an electrical one. The optical source can be either mounted inside the packaging of the MEMS device or outside the package. Because most MEMS devices are fabricated on silicon, the laser diode will most likely be hybrid mounted on the chip or with the MEMS package. An external light source implies that the optical waveguide which carries the output signal will be attached via fiber pigtail which will extend outside of the MEMS package so that some external laser light can be coupled into the device. The optical detector may be 
integrated on the same substrate as the MEMS device, or hybrid mounted externally, where once again the output waveguide is extended beyond the package as an output of the chip.

The integration and coupling of lasers and detectors to integrated waveguides has been studied by other researchers and is not the focus of this research, instead the objectives in this research will focus on the optical waveguides which will be used to introduce, collect and measure the motion of a MEMS device [41, 42, 43, 44, 45].

Using integrated waveguides to perform optical metrology has several advantages over a BOM method which utilizes optical fibers. Primarily, integrated waveguides will create a method for measuring device behavior in-situ. A second advantage to integrated waveguides is the permanent mounting to the MEMS substrate. Properly designed waveguides fabricated next to the MEMS devices on the die surface will maintain alignment for the lifetime of the device. Because they are integrated, the optical waveguides can have dimensions that are on the order of magnitude of the MEMS devices or smaller. If optical sources and detectors are fabricated on the chip, then the entire optical measurement system can be integrated. Integration not only miniaturizes the optical probes; but, if the development process of the optical waveguides is performed using standard techniques for microelectronic fabrication, the waveguides can be mass produced in methods similar to the MEMS fabrication. Co-integration of the two technologies will allow the probing of multiple structures simultaneously. Proper packaging of the waveguides will allow the user to monitor the performance of the MEMS devices during operation without interrupting the function of the device [30].

Currently MEMS devices are being co-integrated with microelectronic circuits that are used to read signals being produced by the devices and to drive the MEMS devices. Co-integration of photonic 
and optical devices with MEMS will further expand the technology base from which system function may be designed.

\subsection{Critical Design Issues for the Co-Integration of Waveguide with}

\section{MEMS Devices}

The most important element in the planar IOM system is the integrated optical waveguide. MEMS devices, optical waveguides and electrical circuitry will be existing on the same substrate, and the objective in laying out the waveguides is to find a geometry which minimizes the real estate used without suffering large optical losses. Interference caused by the waveguide with the location of electrical wiring is an issue of routing and is not addressed here, because routing is application dependent and wiring density is typically not an issue in MEMS. Given that a comb drive linear resonator has been chosen as the experimental device, the layout of the optical waveguides has been constrained to some extent. However, the basic principles in waveguide layout and fabrication can be related and transferred to different types of measurements on other types of MEMS devices.

In BOM, the alignment of the optical fibers was a critical issue in achieving a good optical signal. In IOM, the ability to maintain alignment is solved, but dependent upon the waveguide design and placement with respect to the MEMS. Given correct design, the alignment will be performed once during fabrication, and the quality of waveguide alignment becomes a process issue. 


\subsubsection{Optical Waveguide Geometry}

The optical probe layout requires two optical paths, one that directs the light from the source to the MEMS device, and a second one which travels from the MEMS device to a detector. Even though there are two optical paths, the design does not require two separate waveguides. Because of optical non-interaction, the light can travel inside the same waveguide.

Two co-integrated waveguide structures that can be used to optically measure the motion of a linear comb resonator structure are shown in Figure 5-1. While the two waveguides demonstrate different geometries for guiding the optical power, they both utilizes similar paths. An optical source can be coupled into one leg of the optical waveguide. The light traverses the guide and after exiting the end face it illuminates the edge of the linear resonator stage. A fraction of the reflected light will be coupled back into the same end face, travel back along the waveguide structure, and then the optical power will be split between the two waveguide legs. The power that transverses back along the non-input leg will be coupled into the detector this optical path is shown in Figure 5-2.

In Figure 5-1 the top waveguide structure would be used for a multimode waveguide layout, and the bottom figure would be used for a single mode waveguide layout. The upper waveguide structure utilizes corner bends to redirect the optical path. The corners are cut at $45 \mathrm{E}$ angels to reflect the light around the right angle bends. While these bends can be created for single mode waveguides, the dimensions are critical for low optical losses. In this waveguide design, the section of the waveguide 


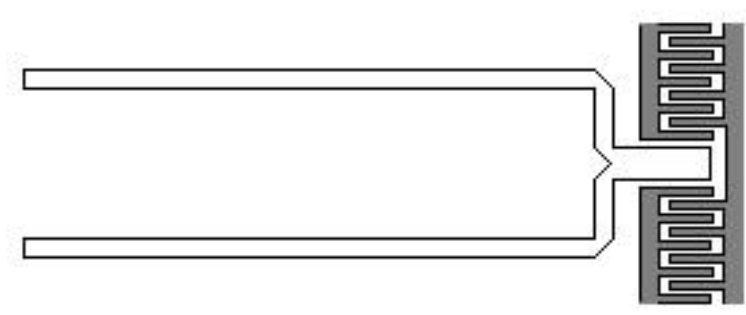

Linear

Resonator

Stage

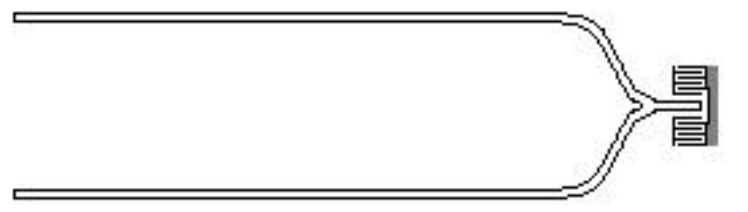

Linear

Resonator

Stage

Figure 5-1: Optical Waveguide Layouts for Probing a Linear Resonaotor.

\section{Optical}

Detector

Optical

Source

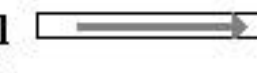

(1)

Stage


probe that will be fabricated next to the linear resonator is twice the thickness of the waveguide legs. The lower waveguide, which maintains uniform waveguide width over the entire optical path, is more suited to a single mode guide layout. The S-bends in the waveguide legs are a gradual method of beam redirection and can be designed to minimize losses in the single mode guides. Because the S-Bends require a large radius of curvature, at least $1 \mathrm{~mm}$, for low losses, the single mode organic waveguide structure will be much wider than the linear resonator stage and flexures [42].

When the linear comb resonator was designed, it was modified with gaps in the comb structures to accommodate the waveguide probe structure. The gap is $140 \mathrm{Fm}$ wide, providing a large space for the waveguide structures. When fabricated the linear resonator stage is fabricated in its natural position, but when operating the stage has a maximum physical deflection of $20 \mathrm{Fm}$ to the left and the right. During operation the linear resonator stage will not achieve this maximum deflection, but the waveguide structure should be aligned a minimum of $20 \mathrm{Fm}$ spacing from the linear resonator stage, which still provides plenty of clearance for additional waveguide processing steps.

\subsubsection{Optical Waveguide Fabrication}

A second issue that will determine the feasibility of using optical waveguides for MEMS metrology is the method by which the waveguides are fabricated. Not only is the influence of fabrication on the optical quality of waveguide a concern, but the compatibility of the optical waveguide cointegration with the MEMS fabrication process will determine the viability of IOM approaches.

The co-integration of the two technologies means that the fabrication steps for fabricating waveguides and fabricating MEMS structures must be interspersed. Surface micromachining has 
become a standard process that utilizes similar techniques to those used to fabricate microelectronic devices. Processes used to fabricate optical waveguides should also utilize standard, VLSI fabrication techniques. Co-integration will also dictate that the waveguide materials and processing techniques be compatible with MEMS processing.

Surface micromachining consists of depositing and patterning alternating layers of polysilicon and silicon dioxide. During each fabrication step the film is deposited by low pressure chemical vapor deposition (LPCVD), then annealed at 1050EC for an hour, and finally is patterned using reactive ion etching (RIE). (See Chapter 8 for a full process description.) During the MEMS processing, the silicon dioxide, which is phosphorosilica glass (PSG) acts as a hard etch mask for the polysilicon RIE. The PSG also acts as a protective coating for underlaying layers of polysilicon and silicon nitride during all etch processes [12].

If waveguides are being co-integrated with the surface micromachining process, the issues of compatibility of the two processes and materials are dependent upon what order the different structures are fabricated. The co-integration of microelectronics and MEMS devices is achieved by fabricating the microelectronic elements first. Then the substrate regions that contain electronic devices are masked to protect the underlying circuitry. Finally, the MEMS devices are fabricated using surface micromachining[21]. Similarly, one method of co-integration would be to fabricate the optical waveguides first. In this pre-MEMS waveguide fabrication, the compatibility issues focus on the exposure of the waveguides to the MEMS processing. Will the waveguides need to be protected? Is the optical material stable at the anneal temperature? Another question to consider is the extra surface topology that the waveguide structures create. 
A second option is to interpose the fabrication steps of the waveguide process with the fabrication steps of the surface micromachining. The entire process would alternate the depositing and patterning of MEMS layers with waveguide layers. An advantage of this method is that the alternating of film depositions will not create excess topology. Because the different layers will be exposed to extra processing steps, issues that face this type of co-integration focus on the compatibility of the materials and processes. What materials will the different films adhere to? Will the materials be resistant to the different etch processes? Can the same materials be used to fabricate both MEMS and waveguide structures?

A third option for waveguide co-integration is to fabricate the optical waveguides last as a postMEMS waveguide fabrication process. In this method, the optical waveguides can be fabricated after the MEMS have been fabricated. The advantage to this processing method is that the waveguides will be aligned to the final MEMS devices. During other processes, the position of the devices may change due to slight changes in position during alignments. The MEMS devices will be protected by a coat of silicon dioxide mask. Is this hard mask significant protection during the waveguide processing? Can the waveguide materials be fabricated over the sacrificial oxide? What extra processing must be performed to deposit optical waveguides?

No matter what process method is used, the ultimate final process step will be the release of the MEMS devices. During the release process the die are submerged in $50 \%$ hydroflouric (HF) acid. This acid bath etches away all the sacrificial silicon dioxides deposited during the micromachining process. Once the MEMS devices are released, they are free moving and they cannot take aggressive forces required for additional processing. Whatever the material choice used for fabricating optical 
waveguides, it must be resistant to the HF bath, or suitably protected with a material that would be a permanent part of the final waveguide-MEMS structure.

\subsection{Optical Waveguide Material Selection}

While the optical waveguide geometry will dictate some of the fabrication criteria, such as feature resolution and film thicknesses, the most important factor that will determine the types of processing steps to be used will be the choice of optical waveguide material. The material will also dictate at which step the surface micromachining process will be interrupted to accommodate the fabrication of optical waveguides.

The choice of optical waveguide material will dictate both the fabrication process and the waveguide geometry. It will help determine the method used to deposit and pattern the waveguides. Because the material may be susceptible to the surface micromachining RIE and the material may not be stable to the annealing temperatures, the material dictates whether the waveguides can be preprocessed, inter-processed, or post-processed with the MEMS devices.

The method of waveguide patterning can also dictate the waveguide cross-section. If the material is etched away, a raised waveguide cross-section, like a ridge waveguide, will be fabricated. If the material is doped or deposited into a recess, then a buried structure, like an embedded strip waveguide, will be fabricated. Another way the material affects the geometry of the waveguide is by its index of refraction. The number of modes supported by a waveguides is dependent upon its dimensions and upon the difference between refractive indices of the waveguide core and cladding. Lower 
differences between core and cladding means that larger waveguides can be fabricated and still support a single mode.

Integrated waveguides are fabricated from thin, optical films deposited on a substrate. In the 1960 's, research in thin film technologies developed the foundation for development of integrated optical technologies in the 1970's. Early integrated passive optical components, which include waveguides, were primarily fabricated in silicon dioxide. Silicon dioxide, as well as silicon nitride, and various metal oxides are classified as inorganic optical films. In the early 1980's research of optical polymers showed they too can be used to fabricate waveguides, and in the early 1990's specially designed optical polymers emerged optimized for low loss waveguide fabrication. The new polymer materials are classified as organic optical polymers [41, 46, 47].

\subsection{Inorganic Optical Materials}

Metal oxide films are normally fabricated on non-silicon substrates for most waveguide applications. The linear resonator will be fabricated on silicon, which indicates using the compatible silicon dioxide and silicon nitride. Both of these materials are already used in the MEMS fabrication process. Silicon dioxide is an excellent optical waveguide material, since it is the same material used to make optical fibers. In the linear resonator fabrication process, silicon oxide is deposited using low pressure chemical vapor deposition. The type of $\mathrm{SiO}_{2}$ being deposited is phosphorosilica glass (PSG). The silicon nitride film is also deposited using LPCVD [12].

In the MEMS fabrication process the silicon nitride film acts as a passivation layer. Nitride is deposited on the surface of the silicon substrate before the MEMS structures are fabricated. This 
surface serves two purposes: one, the nitride is a dielectric and it electrically insulates the MEMS structures from the substrate, and two, the nitride acts as an etch stop during the HF bath, since it is chemically resistive to $\mathrm{HF}$.

While silicon nitride has been used to fabricate waveguides, they are not suited for this application. $\mathrm{Si}_{3} \mathrm{~N}_{4}$ of the density used has an index of refraction of 1.94; $\mathrm{SiO}_{2}$ has a refractive index of 1.45. A silicon nitride waveguide core can be surrounded by a silicon dioxide cladding, but the index of refraction difference between the two materials is quite large. To create a single mode waveguide, the core must be extremely small, less than $0.28 \mathrm{Fm}$. A second property of nitride films that is undesirable is that they are often birefringent.

A better solution would be to make a silicon dioxide waveguide core with silicon dioxide cladding. $\mathrm{SiO}_{2}$ can be doped to adjust its index of refraction. PSG is doped with phosphorous or Boron. This can be achieved with thermal diffusion or ion implantation in already fabricated films. More uniform doping may be achieved by adjusting the gas percentages during LPCVD. During the MEMS surface micromachining, the devices are annealed at 1050EC several times. One purpose of the annealings is to allow the PSG to dope the polysilicon. This annealing temperature could cause diffusion in the waveguide structure, which can create graduated index changes across the waveguide boundary. A second way to adjust the index of refraction of the core is to deposit silicon oxynitride. This hybrid film can have an index of refraction that ranges from 1.5 to 1.98 , depending on the oxide to nitride ratio. SiON is deposited by LPCVD, and the blend percentage is controlled by gas flow. In this case a silicon oxynitride core, with an index of 1.5 could be clad with a silicon dioxide, with and index of 
1.45. The index difference between the core and cladding is 0.05 , and the core dimension for a single mode $\mathrm{SiON}$ waveguide would be $2 \mathrm{Fm}$ operating at a wavelength of $1300 \mathrm{~nm}$ [48, 49].

Using inorganic waveguides, a single mode, or multimode waveguide structure could be fabricated. Because the waveguide materials are fabricated out of the same materials used in the surface micromachining process and use the same deposition methods, the fabricating of the waveguides could be interposed with the fabrication of the MEMS devices. The MUMPS process at MCNC uses an oxide layer of $2 \mathrm{Fm}$ for the first oxide spacer and an oxide layer of $0.75 \mathrm{Fm}$ for the second. Both dimensions are sufficient to act as an upper and lower cladding. Using the first oxide layer as a sub cladding for the waveguide, will ensure that the waveguide core and stage of the linear resonator, which is fabricated in the poly1 layer, will be aligned. Unfortunately the oxide layers deposited during the MEMS device fabrication are used as sacrificial material. After the entire process is complete, the MEMS devices are inserted into an HF bath for the purpose of removing all the silicon dioxide material. If silicon dioxide is going to be used as a waveguide material for the co-integration process, then the waveguide structures must be protected from the HF bath. The protective material which is used to encapsulate the waveguides, must be optically transparent, since there will be no additional processing after the devices are released, the encapsulation material will not be removed.

A thin film of silicon nitride, approximately $0.25 \mathrm{Fm}$ thick, can be deposited over the entire waveguide structure using LPCVD to encapsulate it. $\mathrm{Si}_{3} \mathrm{~N}_{4}$ is optically transparent and it is resistant to HF. Figure 5-3 is a cross-section of this proposed waveguide structure aligned to a fully released resonator. The thickness of the nitride encapsulating layer would be adjusted for maximum transmission at the waveguide endface. 

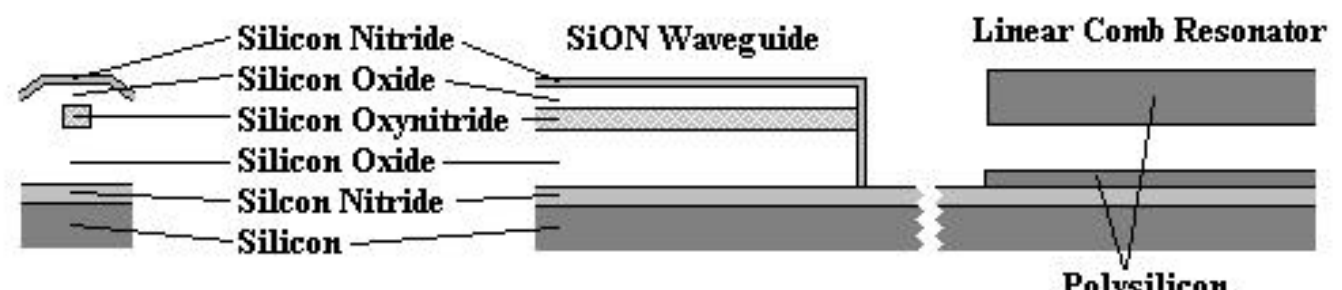

Figure 5-3: Cross Section of Inorganic Waveguide and Linear Comb Resonator

\subsection{Co-integrated Fabrication Process Utilizing Inorganic Waveguides}

Given these considerations, Figure 5-4 shows a proposed process flow for the co-integration of silicon oxynitride waveguides with the linear comb resonator. This process is a modification of the MCNC MUMPS surface micromachining process. The waveguides

achievable with this process will be modeled and simulated in the next chapter with respect to their optical properties.

The first step of the process is to deposit a 0.6 Fm layer of silicon nitride using LPCVD on a clean silicon substrate. Step two is the deposition of the poly0 layer. A $0.5 \mathrm{Fm}$ layer of polysilicon is deposited onto the wafer surface using LPCVD. The poly0 layer is reactive ion etched to pattern the MEMS device ground plane. A 2 Fm film of PSG is deposited using LPCVD and patterned using RIE. This film will act as both a sacrificial oxide layer, Oxide1, and as the subcladding for the SiON waveguide core. The next MEMS fabrication process is to deposit a $2 \mathrm{Fm}$ thick film of polysilicon by LPCVD. This film is then covered with very thin coat of PSG, 0.2 Fm thick, and annealed at 1050EC for one hour. The PSG film serves two purposes. During the anneal, the PSG acts as a doping source of phosphorous for the polysilicon layer. Its second application is to use it as a hard etch mask for the 
patterning of the polysilion. After the anneal process, a coat of photoresist is applied to the PSG, and it is patterned with the polysilicon mask. A RIE is used to pattern the PSG, a second RIE is used then to pattern the polysilicon. After the polysilicon etch the resist is stripped off the PSG surface. The next process step would be to strip the $0.2 \mathrm{Fm}$ layer of PSG using a RIE. To protect the subcladding layer of silicon dioxide, an interruption in the standard surface micromachining process is created by applying a new photoresist mask to protect the silicon dioxide cladding. This is shown in step 5 of Figure 5-4.

Once the PSG is removed the photoresist can be removed from the cladding. At this point, a 0.88 Fm layer of silicon oxynitride can be deposited using LPCVD. The waveguide core can be patterned using RIE. A second layer of PSG is deposited to create the Oxide 2 layer and the top cladding of the waveguide structure. The deposited silicon dioxide is $0.75 \mathrm{Fm}$ thick, and it can be patterned using RIE. After patterning the Oxide 2 layer, a thin film $(0.25 \mathrm{Fm})$ of silicon nitride is deposited over the entire surface using LPCVD to encapsulate the waveguide structure. It will then be patterned using RIE. The linear comb resonator is then metalized.

Finally the MEMS structure can be released by submerging the entire die in a $50 \%$ HF bath for two and a half minutes. The die are then rinsed in DI water, soaked in alcohol to displace the DI water under the devices and baked at 100EC for thirty minutes to dry the MEMS structure. 


\section{PolySi $/ \mathrm{Si} \square \quad \mathrm{Si}_{3} \mathrm{~N}_{4} \square \quad \mathrm{SiO}_{2} \square \quad$ SiON $\square$ Photoresist $\square$}

Step 1: Deposition of $0.6 \mu \mathrm{m}$ layer of silicon nitride.

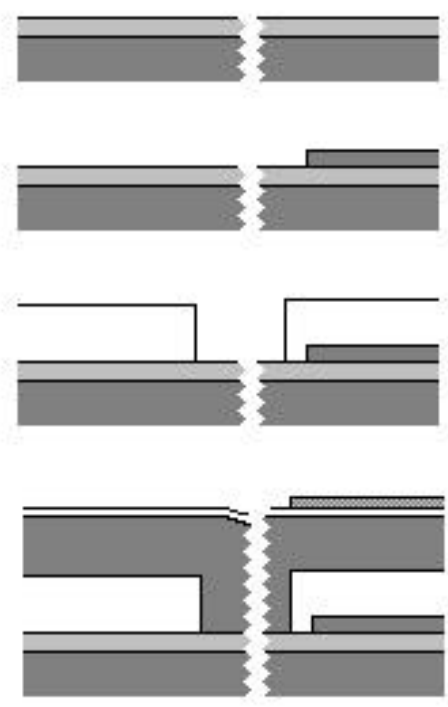

Step 4: Deposition of $2 \mu \mathrm{m}$ layer of polysilicon and $0.2 \mu \mathrm{m}$ layer of PSG and anneal. Deposition and patterning of photoresist for Poly1.

Step 5: Pattern PSG etch mask. Pattern polysilicon for Poly1. Strip photoresist. Apply new photoresist mask to protect subcladding.

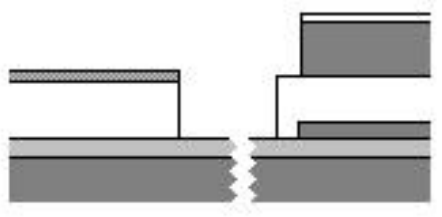

Step 6: Strip PSG and Strip photoresist.
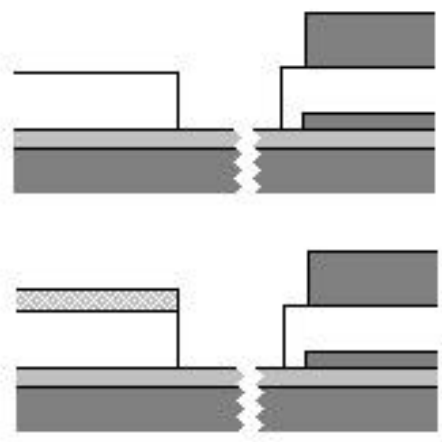

Step 7: Deposit and patterning $0.88 \mu \mathrm{m}$ layer of silicon oxynitride for waveguide core.

Figure 5-4: Co-Integration of Inorganic Waveguide with MEMS Process Flow (Part 1). 
Step 8: Deposit and patterning of $0.75 \mu \mathrm{m}$ layer of Oxide2 and top cladding.

Step 9: Deposit and patterning of $0.25 \mathrm{~m}$ layer of silicon nitride for waveguide encapsulation.
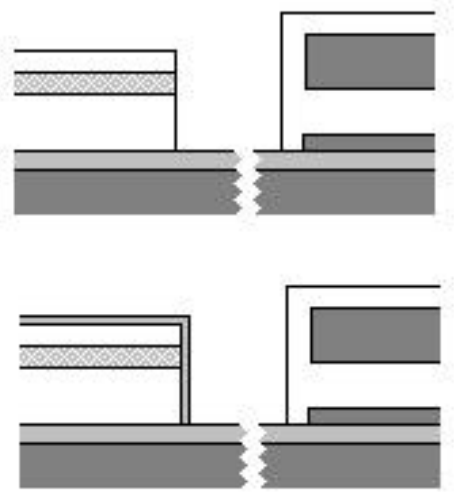

Step 10: HF release.

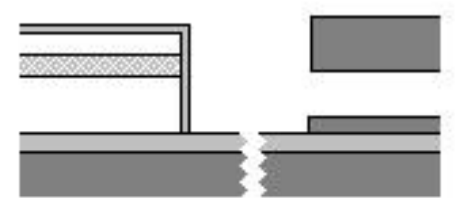

Figure 5-4: Co-Integration of Inorganic Waveguide with MEMS Process Flow (Part 2).

\subsection{Organic Optical Materials}

The use of optical polymers to fabricate waveguides is relatively new when compared to inorganic materials. In the 70's, integrated waveguide technologies expanded rapidly with the development of fiber optics and like the fibers the original integrated waveguides were made from $\mathrm{SiO}_{2}$. In the 80 's, polymers became a material of interest for waveguide fabrication. In the early 80 's research began using polymethyl methacrylate (PMMA) and polystyrenes, which were also being explored for polymer optical fibers. In the mid 80's, two polymer families, polyimides and polysilynes gained favorability, because they were photopatternable $[46,47,50,51,52]$. 
Optical polymer materials attained interest as a waveguide material in electronic and photonic circuits. They can be applied to silicon and III-V materials after all processing is completed. Because they do not require high temperature processing, polymer processing is benign to the integrated circuitry. In addition, polymer chemistry can be engineered to change both its physical and optical properties. Even though polymers have been researched over the past 20 years, there is no one particular polymer that is ideal for fabricating optical waveguides. Selecting a polymer consists of searching for an optical polymer which has the right balance of mechanical and optical properties for a particular application [53].

Polymers are a large group of materials that form long chains for chemical structures. Methane rings are usually attached to the backbone of the polymer, which usually, but not necessarily, contains

carbon; therefore, they are organic materials. The term optical polymers refers to particular families and types of polymers that exhibit certain universal traits that make them favorable for creating dielectric, optical waveguides. These polymers are transparent in the visible and/or infrared wavelength range. They also have a high index of refraction which is necessary for optical waveguiding. Optical polymers must also have low propagation losses over short distances [54].

\subsection{Optical Polymer Characteristics}

While there are different optical polymer families, all optical polymers share some common traits which dictate the use and application of the material. Some of these characteristics are often presented as negative properties. These characteristics are not drawbacks to the material if the proper choice of

processing and handling is utilized with the fabrication of polymer optical waveguides. Like all materials, 
polymers have certain characteristics that impact processing. Traditionally polymer processing is relegated to the end of the fabrication process. This is due to the thermal stability of the materials.

Most optical polymer have a low glass transition temperatures, $T_{g}$, which is typically in the range of $300 \mathrm{EC}$ and $400 \mathrm{EC}$. The glass transition temperature is the temperature at which the polymer will chemically transform from an amorphic polymer to a glass-like material. Traditionally polymers are cured at temperatures just below $\mathrm{T}_{\mathrm{g}}$, because the chemical change in the structure of the material changes its optical loss properties due to ordering and its resulting optical scattering. In typical waveguide microfabrication processing, where growing and diffusion temperatures can reach 1000EC, polymers are considered a low temperature tolerant material $[51,53,55]$.

The conventional and most convenient method for fabrication waveguides is spin-on application. Depending on the substrate size, the polymer may be dipped, sprayed or spread. While polymer films can be deposited by sputtering, the process of sputtering dielectrics can be difficult and this deposition method only increases the complexity of the processing. Normally optical polymers are suspended in solvents to create thick, viscous suspensions that can be applied to the substrate. Afterwards, the films are then baked at a temperature near the glass transition temperature to harden them by driving off the solvents. Using these methods of application may be convenient, but they do not normally achieve thin polymer films. Most spun on films can be 1 to $2 \mathrm{Fm}$ or greater. While not impossible, these minimum thicknesses make it difficult to achieve single mode waveguides. Thinner films may be achieved by sputtering or by diluting the application suspension with more solvents. However the complexities created by either of these solutions is usually not worth the trade offs when other, inorganic options are available. 
The application of polymer films using the spin-on method does require certain considerations. Polymer suspensions can be viscous, and require extra care in handling to avoid the introduction of air bubbles during application. Trapped bubbles in a film can create optical scattering in waveguides. A second issue that must be considered is the speed of the annealing process. Thicker polymer films need time to properly anneal. When polymer films are heated the surface tends to harden quickly, when baking thicker films, the surface can harden before all the solvents are driven out of the film, and when the remaining solvents try to escape they will crack the polymer film. This problem only occurs in thick films [56].

Because polymer films are provided in a solvent suspension, the soft, unannealed polymers are susceptible to solvents. This means special care must be taken when handling unbaked films, and even after baking, polymer films are susceptible to long exposure to solvents. This processing limitations means that most optical polymers are not compatible with photoresist, since it is a solvent suspended polymer. The soft, unbaked optical polymers are also susceptible to $\mathrm{H}_{2} \mathrm{O}$, which if absorbed greatly increases the optical losses of the polymer films. While a soft polymer film can be protected from direct contact with water, care must also be taken when the films are heated. During the baking processes, the films become heated and want to react with water in the atmosphere. To stop this absorption, optical polymer films should be heated in a non-reactive environment, so the furnace is typically purged with nitrogen, any times the polymer films are subjected to a softbake or hardbake. When the films are softbaked on a hotplate, the heating procedure occurs so fast that the water absorption is not usually an issue [57]. 
These are only general sweeping traits of optical polymers that most display. Additional characteristics of individual polymer types must be taken in consideration when selecting a polymer for a particular waveguide application. The evaluation of optical polymers requires examining the mechanical and optical characteristics of a polymer, and each type of polymer has its own unique characteristics. When examining the mechanical characteristics, one must consider the method of application. Most polymers are applied using a spinner, however there are other questions that must be answered in regard to application. Does the polymer require any special substrate preparation, like an adhesion promoter?

A second issue is the patternability of the material. Some polymers are photosensitive and act like positive or negative resist. Other polymers require patterning by a dry etch, and yet other polymers require doping to define waveguides. The type of patterning can dictate what shape of waveguides can be made; whether, the waveguides are strip, rib, embedded strip, etc. Some of the optical properties that should be considered are the value of refractive index, the optical region of transparency, the optical absorption losses, and birefringence. All of these factors can have direct control over the quality of waveguides that can be fabricated.

\subsection{Polymer Waveguide Co-Integration}

Optical polymers can be used to fabricate multimode waveguide probing structures. One great advantage of fabricating waveguides from polymers is that they can be co-integrated in a post-MEMS process. If an $\mathrm{HF}$ resistant polymer is used, then the devices do not have to be protected from the HF release bath. A cross section of a polymer waveguide integrated with a fully released, linear comb 
resonator is shown in Figure 5-5. The thickness of the films limit the waveguide to design to multimode, which will simplify processing since the upper part of the waveguide core can be clad by air. A process flow for the co-integration of polymer waveguides is shown in Figure 5-6. Unlike the inorganic process, where the waveguides were fabricated simultaneously with the MEMS structures, the organic process is performed after the MEMS structures have been fabricated. Before applying the polymer, the PSG that covers the waveguide application area is removed using a buffered oxide etchant. The die is then cleaned in a solvent bath. An adhesion promoter is spun-on the die to help the cladding polymer adhere to the silicon nitride.

For the case of a photopatternable polyimide waveguide, a cladding polymer can be applied using a syringe and spun-on to a thickness of $2 \mathrm{Fm}$. The cladding film is then patterned by exposure to UV light, and solvent development. Once patterned the polymer is baked hard. A second polymer, the waveguide core polymer can be spun-on to a thickness of $2 \mathrm{Fm}$, and then
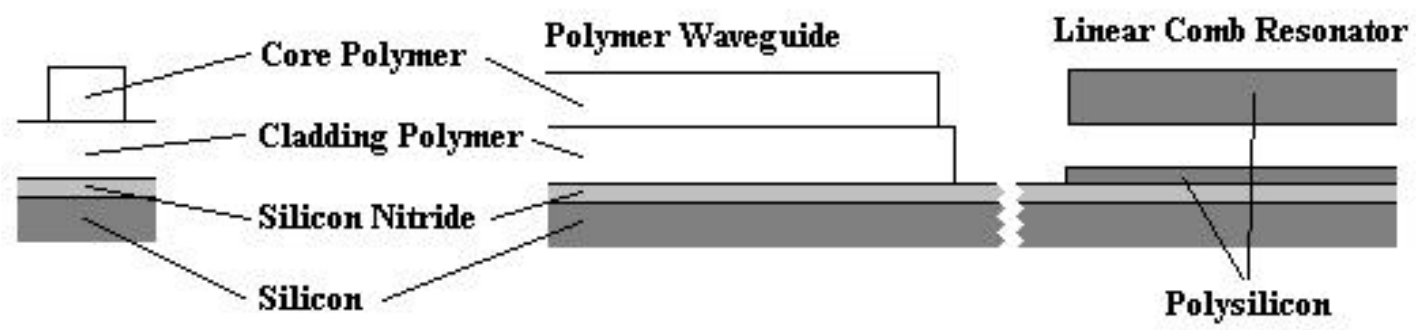

Figure 5-5: Cross Section of Organic Waveguide with a Fully Released Linear Comb Resonator. 
Step 1: Fabricate MEMS structure.

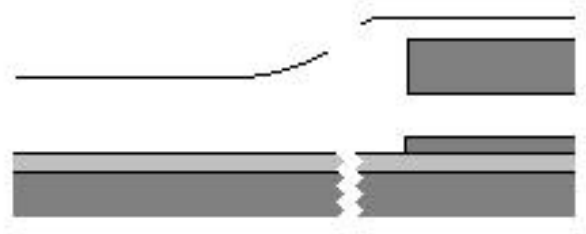

Step 2: Remove sacrificial oxide from waveguide cladding area.

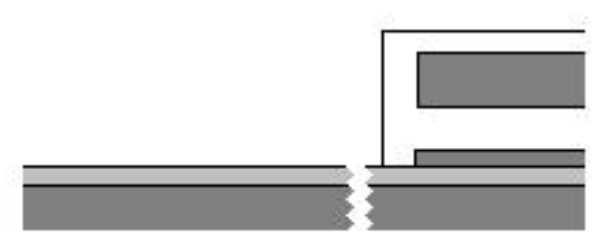

Step 3: Spin-On and pattern waveguide cladding polymer.

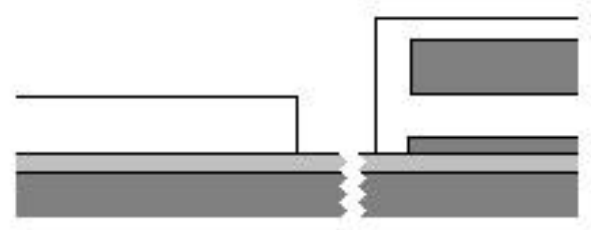

Step 4: Spin-On and pattern waveguide core polymer.

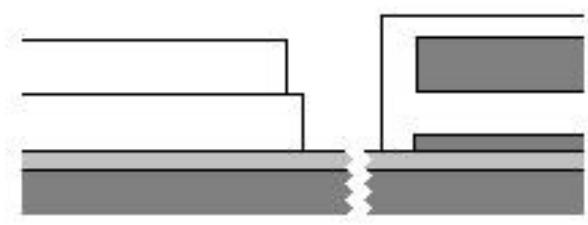

Step 5: HF release.

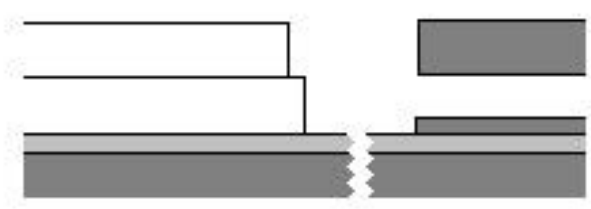

Figure 5-6: Organic Waveguide Co-Integration with Prefabricated Linear Resonator Comb Device. 
exposed, developed and baked. Once the waveguide core polymer is properly hardened, the entire die can be submerged in and HF bath for the linear comb resonator release.

\subsection{Inorganic and Organic Waveguide Applications}

Two methods of fabricating the integrated opitcal metrology probe have been presented. The performance of these two different waveguide designs will be discussed in Chapter 6 and 7 . Each material has advantages and disadvantages. Silicon dioxide/ silicon oxynitride waveguides must be used if a single mode waveguide is desired for the probing application. However, the design and fabrication process must be performed simultaneously with the MEMS development process. Because the waveguides are susceptible to the HF release process, the inorganic waveguides must be encapsulated with silicon nitride.

Being performed after the fabrication of the MEMS structure, the polymer waveguide fabrication does not impact the MEMS process schedule and therefore may be more readily accepted as a co-integration process. An added advantage of post fabrication is that the guides are aligned to the fully fabricated MEMS structures, and the polymer is HF resistant. Only multimode waveguides will be fabricated using the polymer materials in this study, so integrated optical metrology measurements will be limited to changes in intensity, while interference effects resulting from device motion cannot be detected. 


\section{Chapter 6}

\section{Inorganic Single Mode Waveguides}

In Chapter 5 a proposed fabrication process was described for co-integrating a silicon oxynitride waveguide with a linear comb resonator. The purpose of this co-integration is to create an optical probe structure that can monitor the behavior of the linear comb resonator during operation. While the inorganic fabrication process can be used to make both single mode and multimode waveguides, the primary motivation for using inorganic materials is the development of single mode waveguides, which are difficult to realize in high temperature stable polymer materials within the highly topographic MEMS environment.

A cross section of the inorganic waveguide is shown in Figure 6-1. Because the waveguides are being fabricated from the same films in the surface micro-machining process, the thicknesses of the silicon oxide claddings are pre-determined. The subcladding of the waveguide is $2 \mathrm{Fm}$ thick. The maximum thickness that the $\mathrm{SiON}$ waveguide can be and remain single mode is $0.88 \mathrm{~F}$ for a wavelength of $632.8 \mathrm{~nm}$. If an optical wavelength of $1300 \mathrm{~nm}$ is used then the film can be a maximum of $2 \mathrm{Fm}$ thick. The upper, $\mathrm{SiO}_{2}$ cladding on the waveguide structure will be $0.75 \mathrm{Fm}$. On top of the cladding is a $0.25 \mathrm{Fm}$ film of $\mathrm{Si}_{3} \mathrm{~N}_{4}$ which encapsulates the waveguides. 

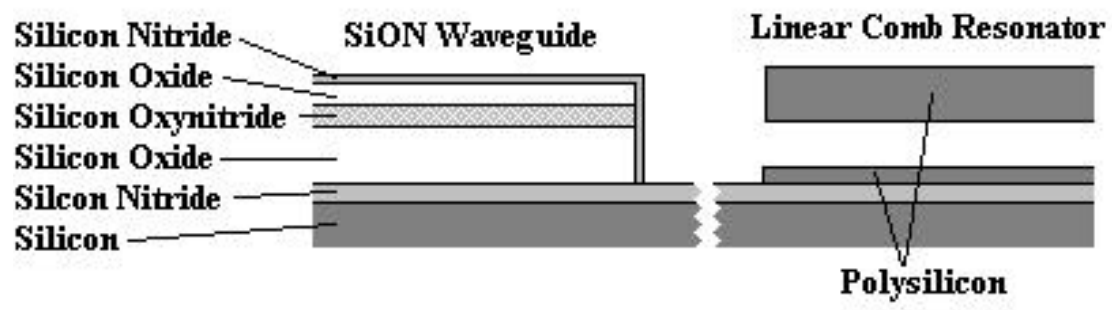

Figure 6-1: Cross Section of Inorganic Waveguide and Linear Comb Resonator.

\subsection{Primary Mode Profile}

The silicon oxide and silicon oxynitride waveguide structure that will be modeled as an imbedded strip waveguide, which is a standard waveguide structure for single mode waveguides. A cross section view of the waveguide structure is shown in Figure 6-2. Silicon dioxide has a refractive index of 1.45 and silicon oxynitride has an index of 1.5 at a wavelength of $1300 \mathrm{~nm}$ [49]. Examining the cross section in Figure 6-2, the structure can be modeled as a 2-D symmetric, slab dielectric waveguide consisting of layers of $\mathrm{SiO}_{2}-\mathrm{SiON}-\mathrm{SiO}_{2}$ along the y-axis. Along the x-axis the structure forms an asymmetric

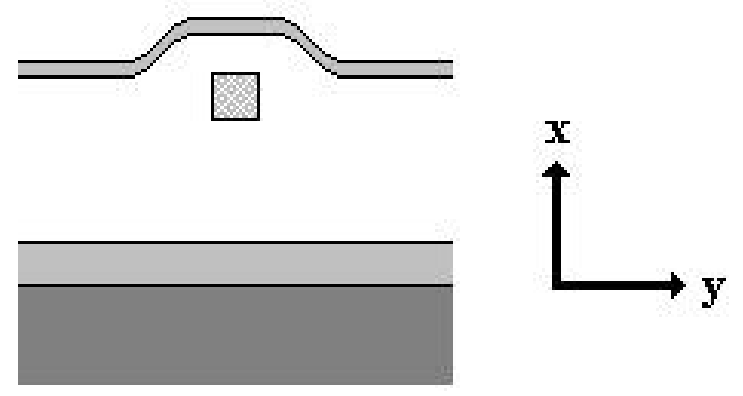

Figure 6-2: Cross-Section of Embedded Strip Waveguide 
waveguide. Modeled as a slab dielectric waveguide the layers would be ordered from the bottom to top as $\mathrm{SiO}_{2}-\mathrm{SiON}-\mathrm{SiO}_{2}-\mathrm{Si}_{3} \mathrm{~N}_{4}$.

Both of these slab dielectric models can be calculated in BPM_CAD 2-D mode solver [58]. Simulations can provide a data graph which gives a visual description of the optical intensity inside the waveguide structure. By varying the thicknesses of the waveguide core in the simulation, the maximum allowable waveguide core dimensions that will support only the primary mode were found. Figure 6-3 shows the mode profile along the y-axis. Here the maximum allowable width of the waveguide core was found to be $1.73 \mathrm{Fm}$, which is slightly different than allowable waveguide thickness which is $2.0 \mathrm{Fm}$. Even in both models the waveguide core is clad by the silicon oxide, the silicon nitride alters the symmetry of the waveguide along the $\mathrm{x}$-axis, which alters the maximum allowable dimension.

\section{Optinal líeld}

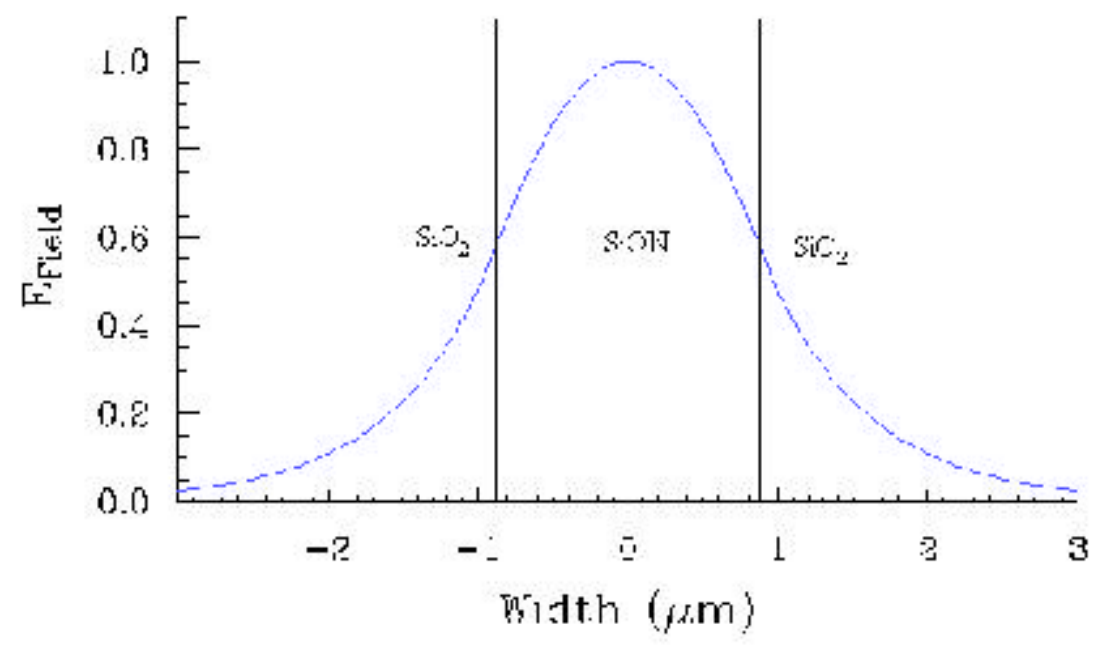

Figure 6-3: Optical Profile of Inorganic Waveguide in y-Direction at a Wavelength of $1300 \mathrm{~nm}$. 
Figure 6-4 shows the optical field profile along the $\mathrm{x}$-axis. The silicon nitride has a comparably high index of refraction, 1.98. Because it has a high index, and is surrounded by silicon oxide, which has an index of 1.45, and air, which has an index of 1 , the thin layer of silicon nitride layer acts as a slab dielectric waveguide. The upper cladding of silicon oxide is only $0.75 \mathrm{Fm}$, and the evanescent field in this cladding layer extends beyond the silicon dioxide and becomes coupled into the silicon nitride. The coupling of the light into the encapsulation material is very small, but over a long distance this amount should increase and create significant loss in the single mode waveguide. However the silicon nitride layer is $0.25 \mathrm{Fm}$, therefore it is single mode. If the nitride carried more modes, it would accept more optical power and the losses in the silicon oxynitride would be more significant.

\section{Optinal lizeld}

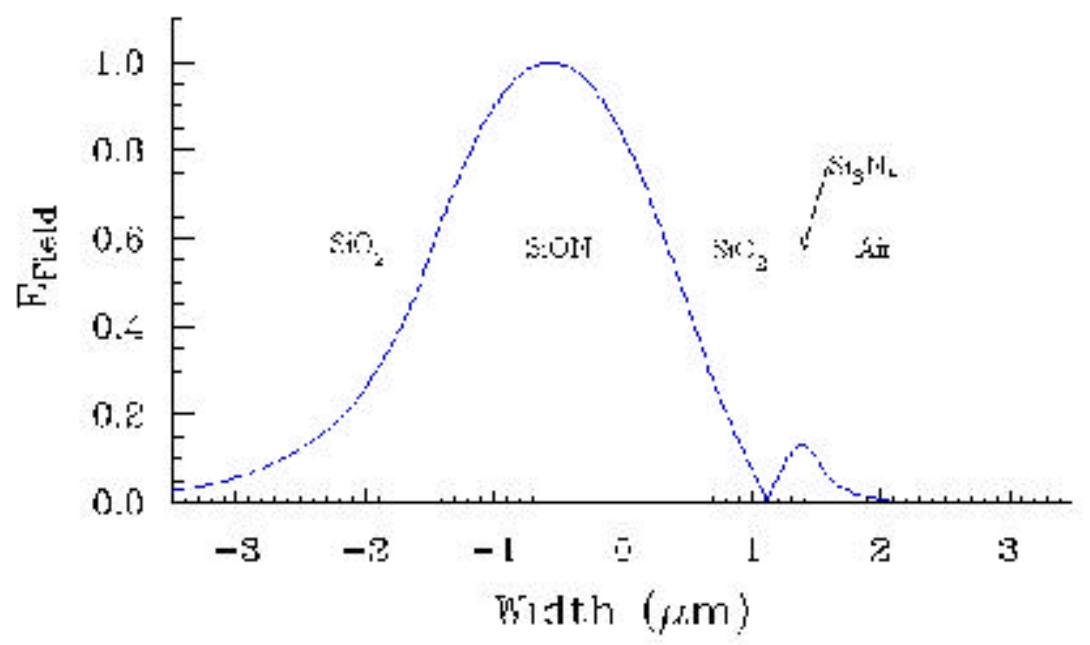

Figure 6-4: Optical Profile of Inorganic Waveguide in x-Direction at a wavelength of $1300 \mathrm{~nm}$. 
If the silicon nitride layer is deposited as a thinner film, less than $1.1 \mathrm{Fm}$, then the encapsulation film can no longer support any modes. This is shown in Figure 6-5. The thickness of the $\mathrm{Si}_{3} \mathrm{~N}_{4}$ is a trade off between ensuring that the waveguides are protected from the HF etch or the encapsulation material coupling optical power out of the waveguide.

To determine if there is significant optical loss over the length of the waveguide, due to the coupling of light into the silicon nitride encapsulation layer, a 2-D optical propagation simulation was performed using an $\mathrm{x}$-axis cross section of the inorganic waveguide. The simulation was performed using BPM 2-D module of the BPM_CAD software. Distribution of the optical field inside and outside of the waveguide structure is calculated using the beam-propagation method. A simulation was performed over a distance of $1 \mathrm{~mm}$. The cross section was modeled by simulating separate waveguides of different indices butted against each other. An output optical intensity profile at $1 \mathrm{~mm}$, with a

\section{Uptinal líeld}

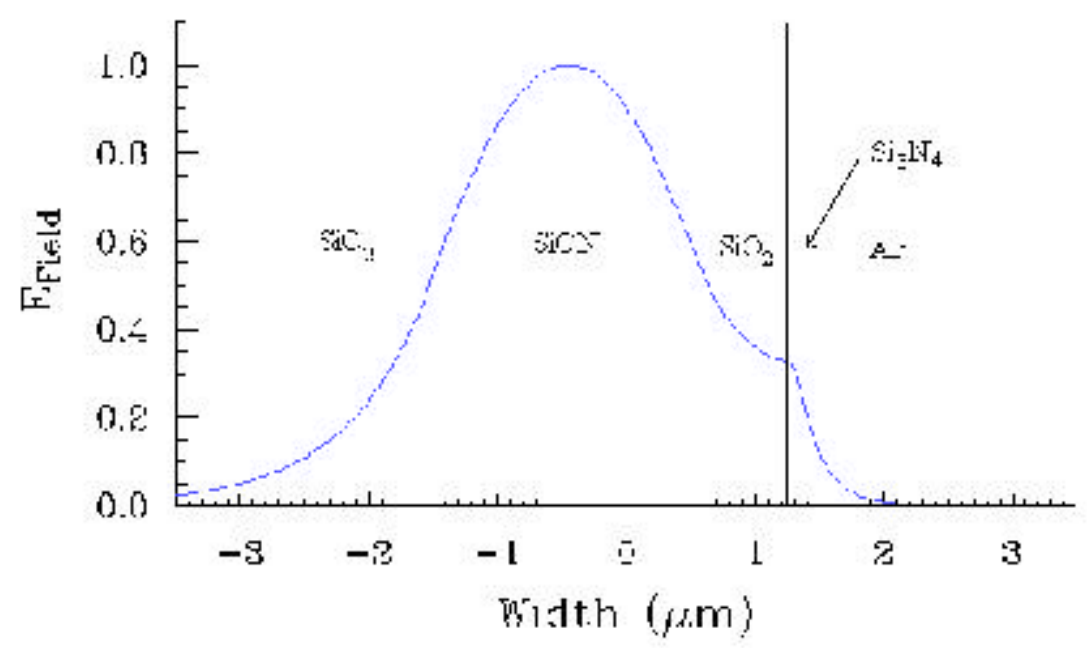

Figure 6-5: Optical Profile of Inorganic Waveguide in x-Direction at a Wavelength of $1300 \mathrm{~nm}$. 
superimposed index profile is shown in Figure 6-6. As shown in Figure 6-7, which is a compilation of the optical field intensity profiles over a distance of $1 \mathrm{~mm}$, there is some small coupling into the silicon nitride layer. The amount of coupling in the encapsulation layer fluctuates, indicate that optical power was being coupled in and out over very short distances.

A simulation was run over a shorter distance of $32 \mathrm{Fm}$ to determine the coupling length, by limiting the simulation to a small distance and performing the analysis over small steps. The resulting compilation of mode profiles is shown in Figure 6-8. The optical power will couple out of the SiON waveguide core, into the $\mathrm{Si}_{3} \mathrm{~N}_{4}$ encapsulation layer and back into the $\mathrm{SiON}$ waveguide core over a length of approximately $8 \mathrm{Fm}$. This coupling distance is very short compared to optical path length over several millimeters.

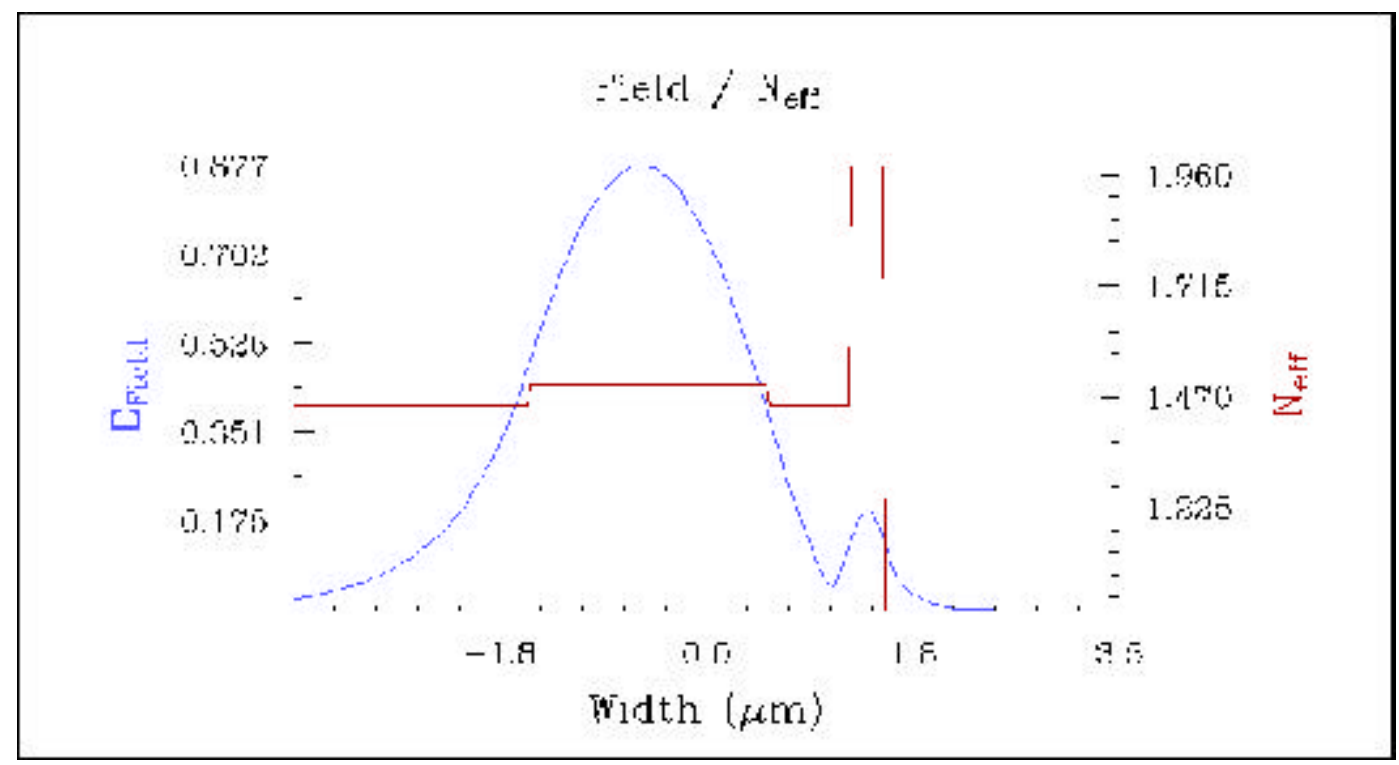

Figure 6-6: Optical Field Intensity Profile and Index Profile at Output. 


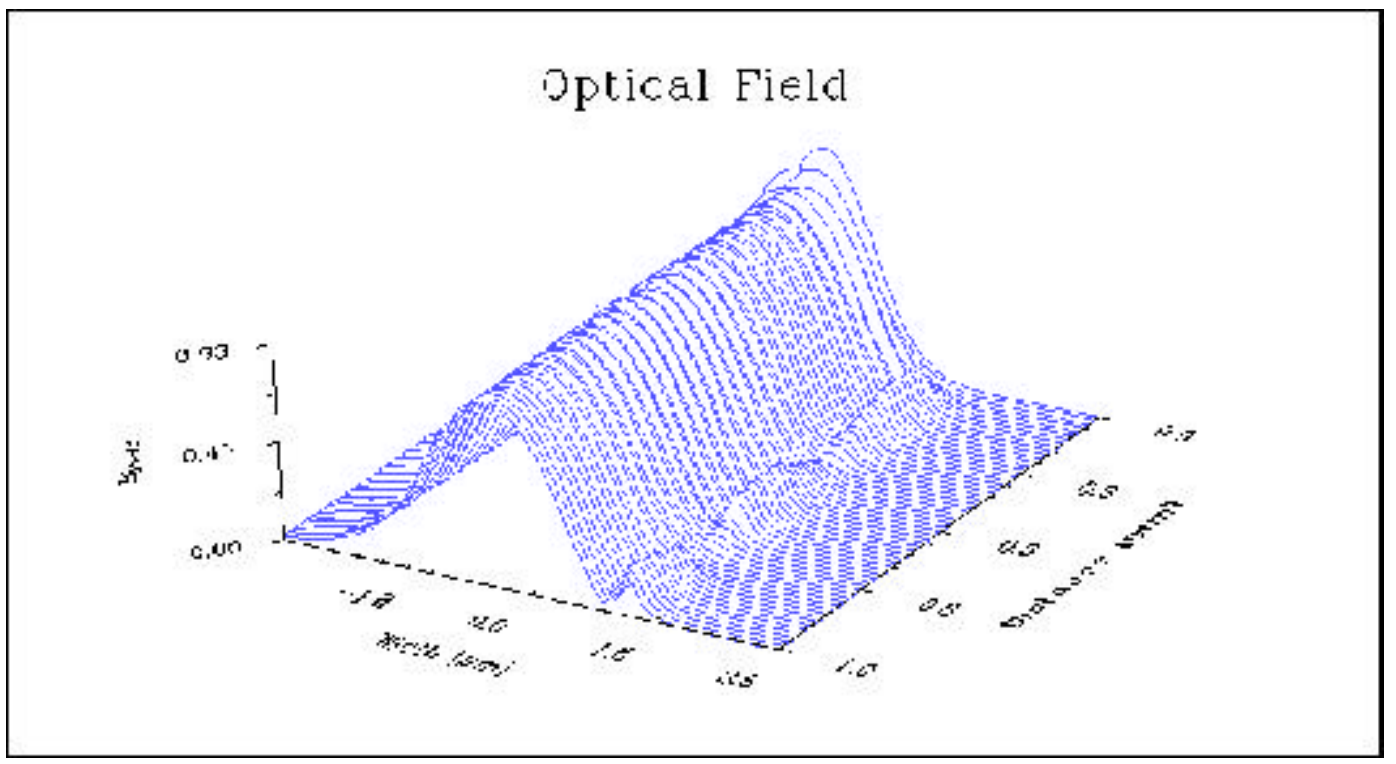

Figure 6-7: Compilation of Optical Mode Profiles over a $1 \mathrm{~mm}$ Propagation Distance.

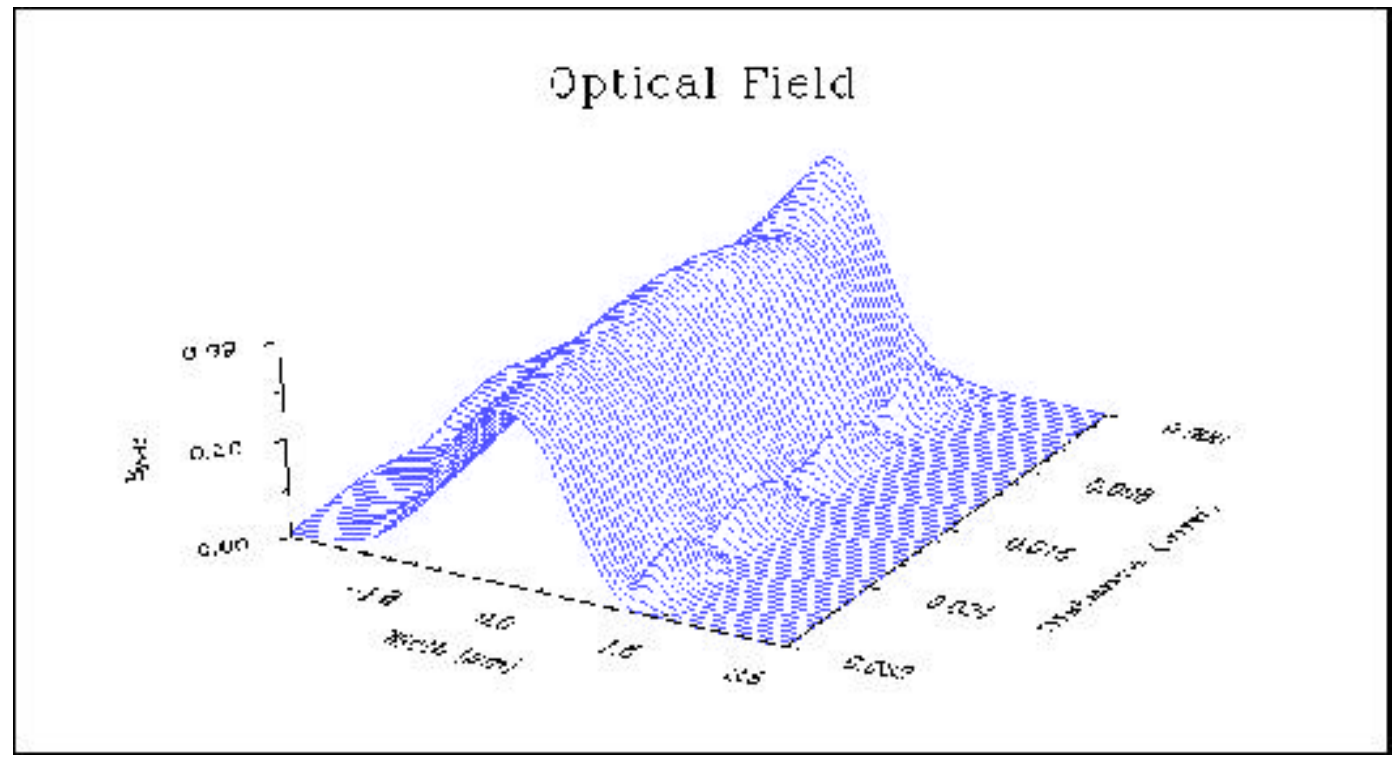

Figure 6-8: Compilation of Optical Mode Profiles over a 32 Fm Propagation Distance. 


\subsection{Single Mode Optical Waveguide Layout for Integrated Optical}

\section{Metrology}

The development of the inorganic single mode waveguide was for the creation of a planar structure that could perform integrated optical metrology. Using single mode optical waveguides to create optical paths for this measurement is desirable if the measurement is to use interference

measurements. Because single mode waveguides emit light that has a coherent field, interference is created between the input light and the reflected light. The changes in optical intensity due to interference can be used to measure very fine distance changes due to high resolution. Using single mode waveguides in this application would create a Fabry-Perot interferometer.

The optical measurement described in Chapter 5 requires a waveguide structure which creates an input path for the optical signal, and waveguide structure which creates an output path for the signal. These two optical paths should overlap for a length of guide where the probe structure is next to the linear resonator. The linear comb resonator has a $140 \mathrm{Fm}$ gap between the combs to allow for the fabrication of the optical probe.

Single mode waveguides carry only the primary mode, and tightly guide the optical power. Sharp bends in the optical waveguide can create significant losses. The layout of the optical probe structure should utilize S-bend waveguides to minimize the optical losses. As demonstrated above, the maximum allowable waveguide width is $1.47 \mathrm{Fm}$. A 3,500 Fm space was left clear of structure to allow for the optical waveguide structure. To optimize the S-bend the change in optical path should be very gradual. Therefore the physical distance between the input waveguide and output waveguide centers was 
chosen to be $10 \mathrm{Fm}$ and the total length of the S-Bend structure is 3,095 Fm. The curve of the S-bend was designed to follow a sine wave curvature. The very tight distances created by fitting the optical waveguide S-bends to very tight spaces create higher losses in the waveguide. Proper design of the waveguides should consider the region where the two S-bends overlap and create a section of waveguide that is larger than the single mode width, and trying to bend the light too fast can lead to high losses and optical fluctuations are demonstrated in Figure 6-9.

Creating single mode layout structure requires finding a balance between good optical characteristics and fabrication dimensions. Single mode guides with low optical losses require S-bends with large radii of curvature on the order of several millimeters, the length of these guides should also be on the order of several millimeters. Shorter guides suffer higher losses and poor

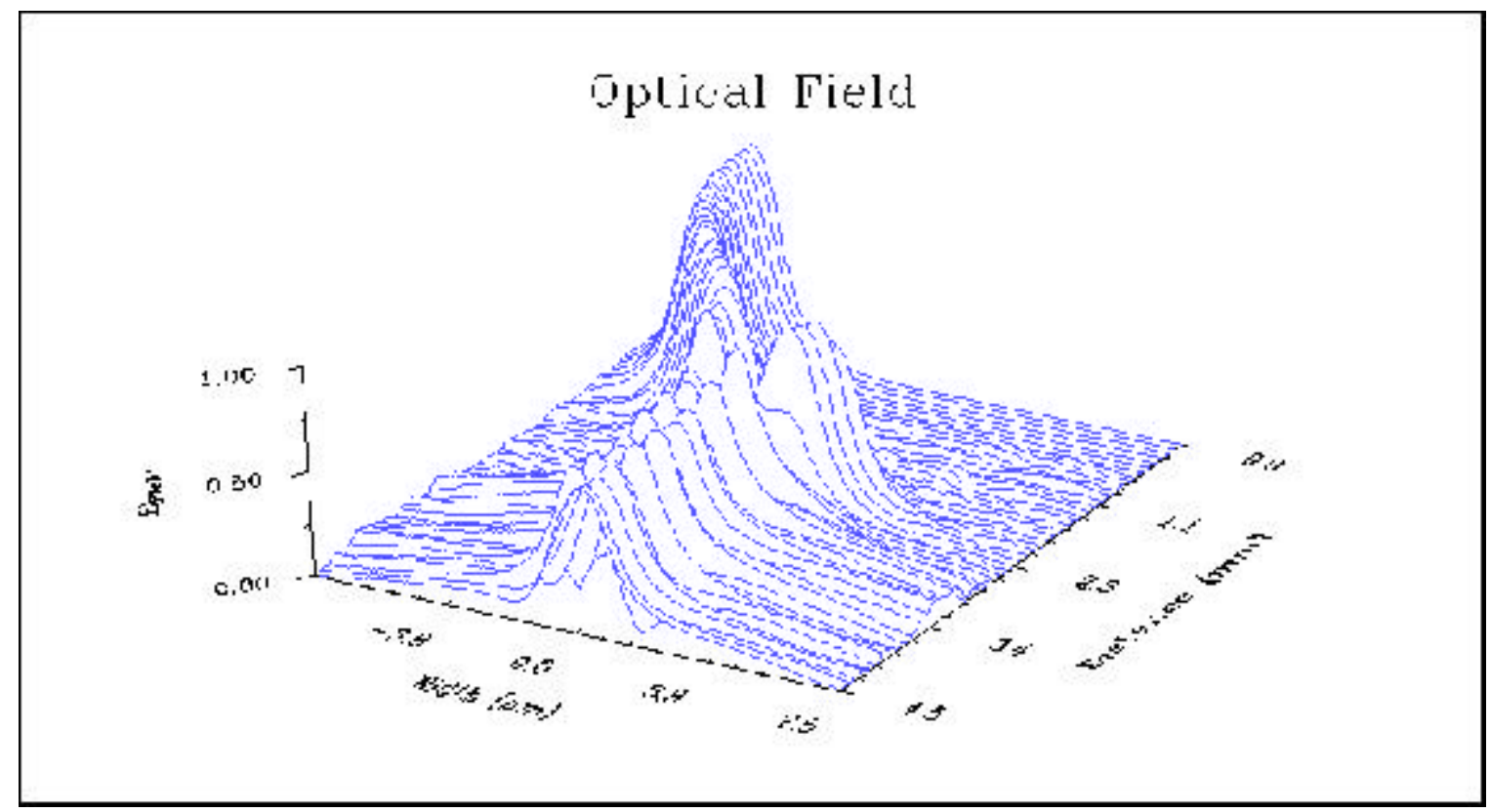

Figure 6-9: Compilation of Optical Mode Profiles over a $0.1 \mathrm{~mm}$ Propagation Distance. 
optical guidance, but MEMS device layouts may not afford long optical waveguide lengths. Figure 6-10 shows the optical field as it travels through a longer single mode guide which contains S-bends that span over $4 \mathrm{~mm}$. The waveguide leg is offset by $15 \mathrm{Fm}$ from the output portion of the guide. This segment is $3 \mathrm{~mm}$ which allows time for the mode fluctuations to settle.

\subsection{Optical Measurement of Linear Resonator Motion}

BPM_CAD is a valuable simulation tool, however it is limited in the types of simulations it can perform [58]. The simulation of optical propagation through a structure occurs only over a positive $\mathrm{z}$ optical path, therefore the modeling tool can not perform back propagation or reflected optical power. To simulate the motion of the linear resonator, it is first presumed that the resonator acts as a perfectly reflecting surface, then the waveguide structure can be mirrored. The distance between the linear resonator is then simulated as a gap between the two waveguide structures. Figure 6-11 shows the

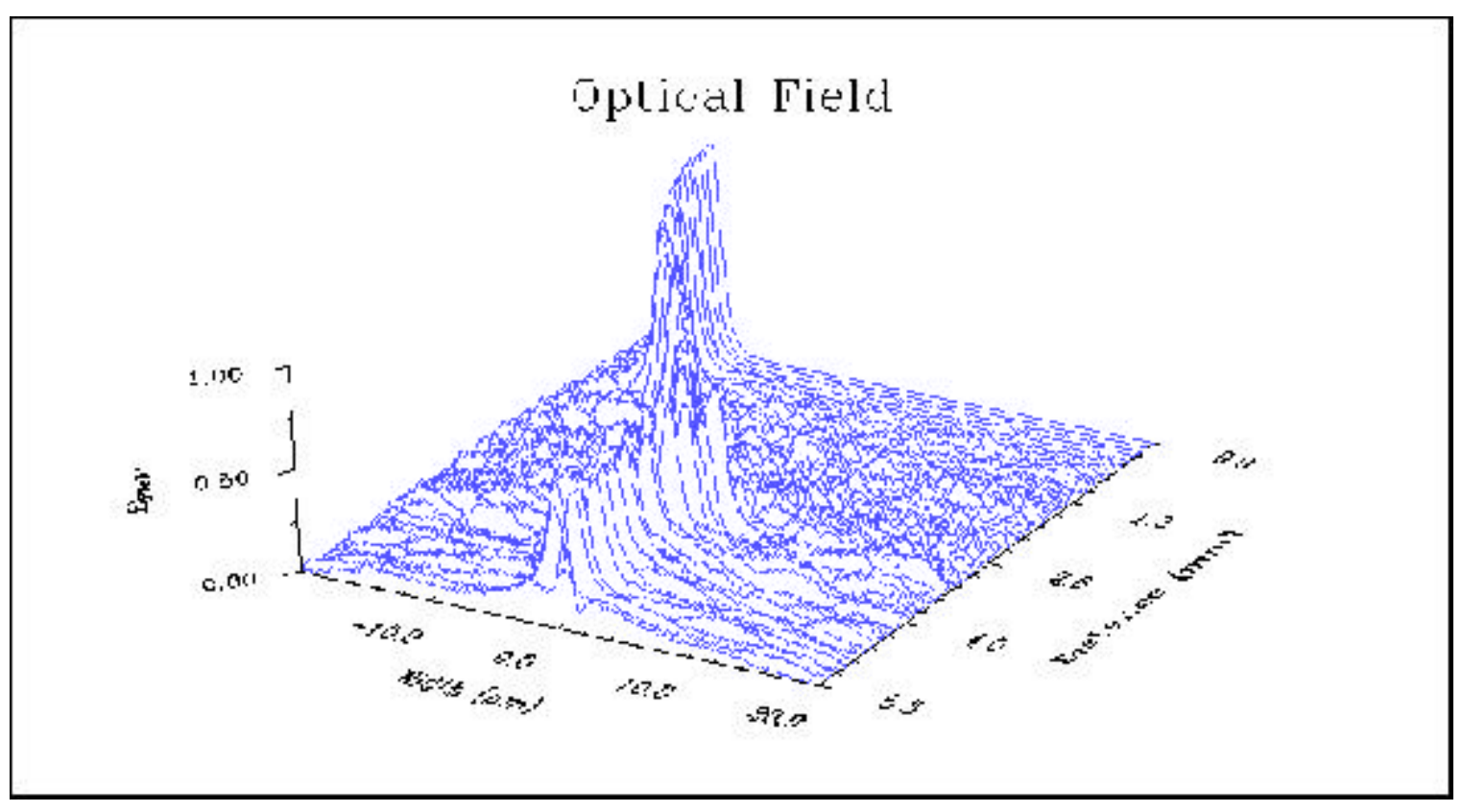

Figure 6-10: Compilation of Optical Mode Profiles over a 5 mm Propagation Distance. 
index of refraction profiles over the distance of propagation for a $20 \mathrm{Fm}$ gap between the linear resonator and optical waveguide.

The calculated optical intensity profiles inside this waveguide structure simulation is shown in Figure 6-12. During the simulation a measurement was made of the total optical intensity output of the right waveguide leg. This value was then normalized by dividing it by the total optical input power into the waveguide. The ratio of optical power out to optical power was collected for several gap sizes.

It was assumed that the end face of the optical waveguide structure was fabricated $20 \mathrm{Fm}$ away from the linear resonator stage. During operation the linear comb resonator stage never reaches the maximum physically allowable deflection, which is $\pm 20 \mathrm{Fm}$. However for the

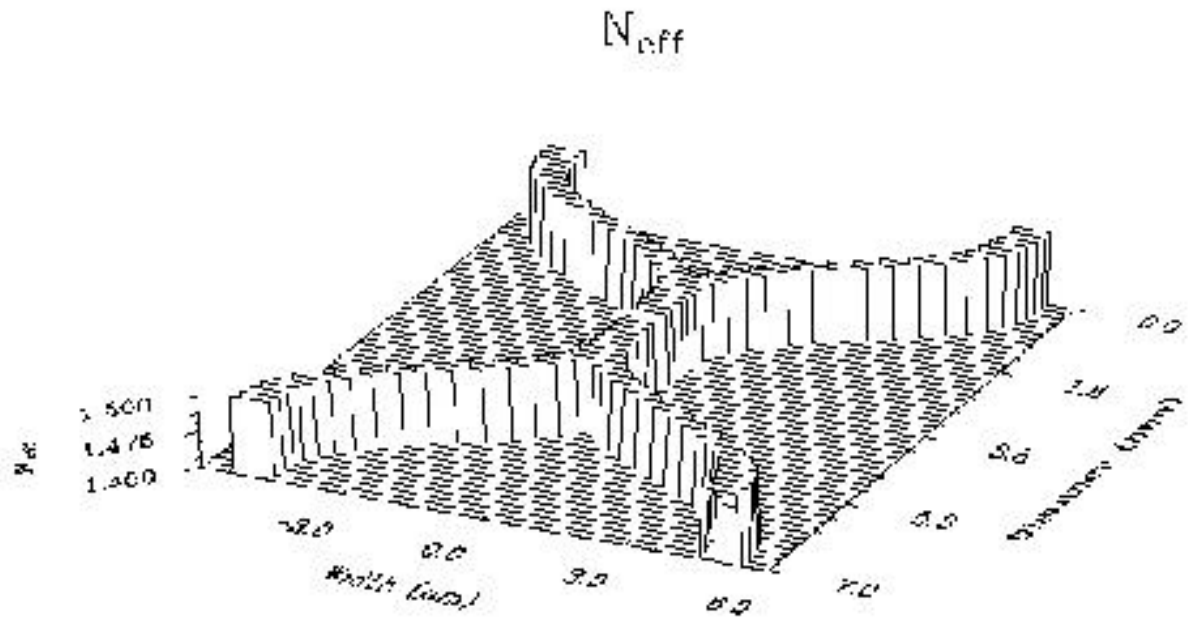

Figure 6-11: Index of Refraction Profile for IOM simulation of 20 Fm gap. 


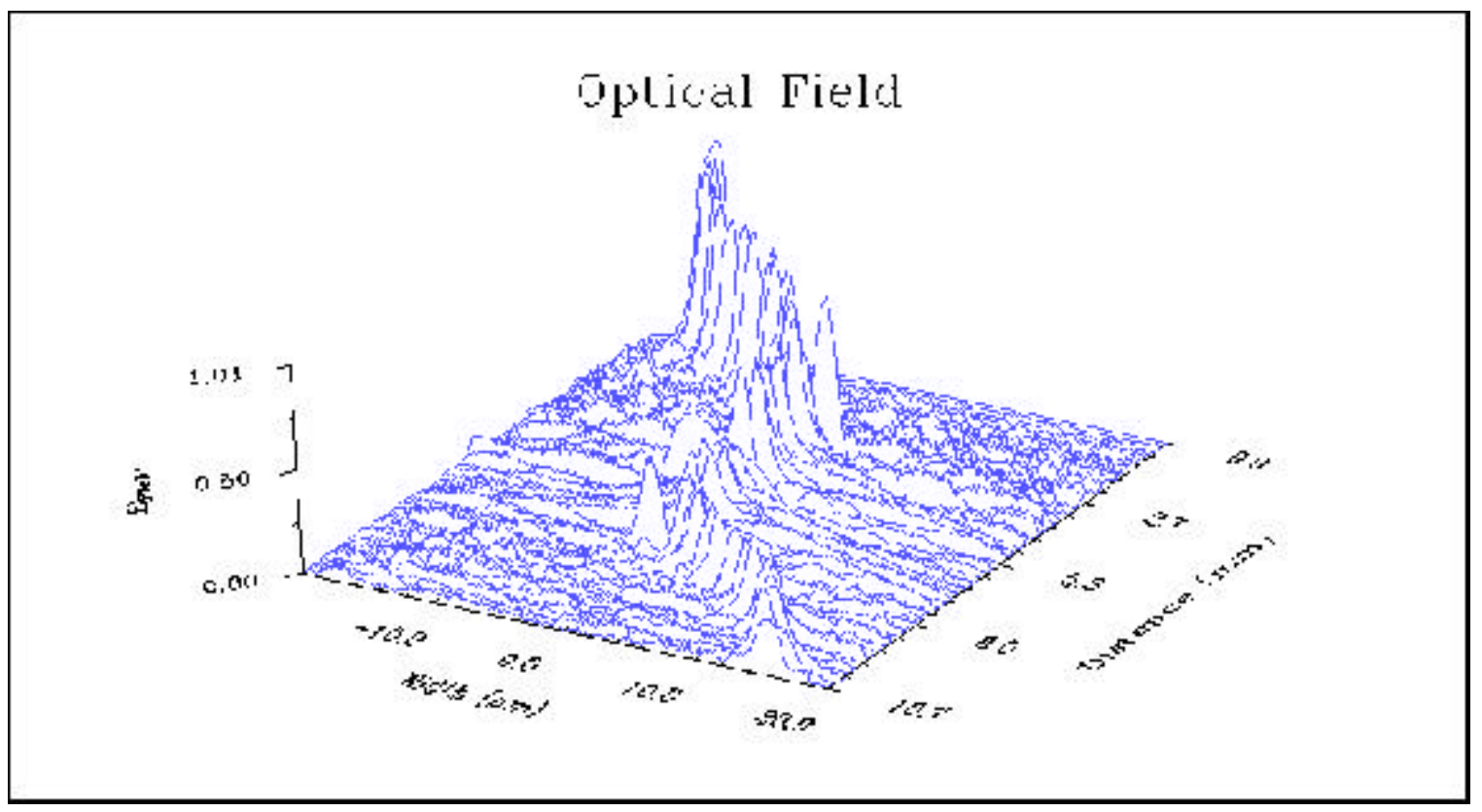

Figure 6-12: Optical Intensity Profiles for IOM simulation of 20 Fm gap.

simulations this gap was assumed to vary from $0 \mathrm{Fm}$ to $40 \mathrm{Fm}$ which represents the entire range of motion for the linear resonator stage. A graph of the optical output ratio versus the deflection distance is shown in Figure 6-13. This simulation only measures the change in optical intensity of the signal to measure the displacement of the linear resonator stage, under the assumption that the linear resonator is a perfectly reflecting surface. In actuality the linear resonator has finite dimensions. Polysilicon, of which the linear resonator is fabricated has a reflectivity of $30.9 \%$. The experimental optical losses in the system are expected to be higher.

As expected the detected optical intensity is not linear. This graph represents the optical intensity as a function of path length, which is an inverse square relationship. 


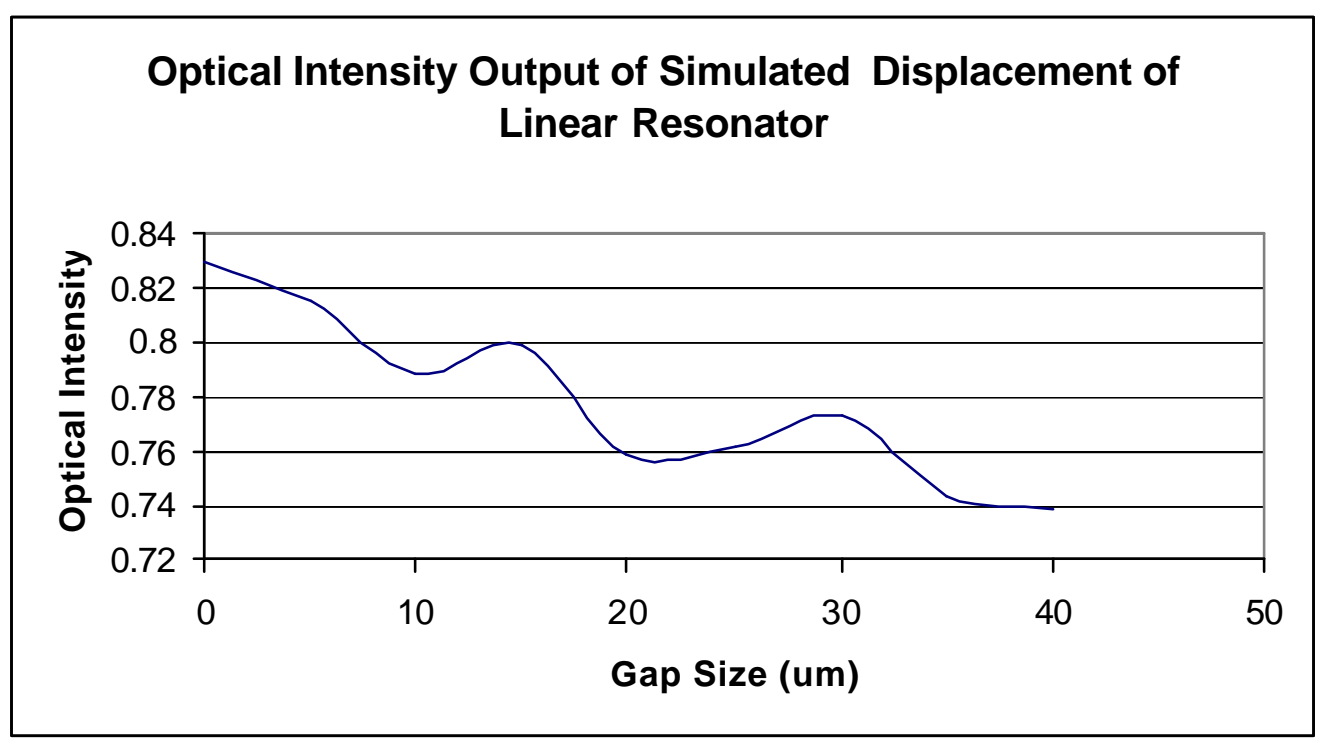

Figure 6-13: Simulated Normalize Output Optical Intensity of a Linear Resonator Displacement Stimulation.

\subsection{Optical Interference Measurement}

The main reason for using a single mode waveguide is to take advantage of the optical

interference effects which occur in the reflected optical signal. Small optical path length differences can create large intensity changes in the output signal. In the inorganic optical waveguide structure, the interference will be created by the phase shifts in the reflected optical signals.

The waveguide core is silicon oxynitride, which has an index of 1.5 . One endface of the optical waveguide probe structure is fabricated next to the linear comb resonator, for the purpose of illuminating the structure and collecting the reflected optical material. This endface is coated with a $0.25 \mathrm{Fm}$ layer of silicon nitride, which has a refractive index of 1.98. For the simulation it was assumed that the endface of the waveguide probe was fabricated $20 \mathrm{Fm}$ from the resonator 
stage edge, and that the maximum deflection of the resonator will be $\pm 20 \mathrm{Fm}$. A picture of the waveguide endface surface, nitride layer, and the linear resonator position is shown in Figure 6-14.

As light exits the waveguide, it encounters three distinct boundaries: the $\mathrm{SiON}-\mathrm{Si}_{3} \mathrm{~N}_{4}$ interface, the $\mathrm{Si}_{3} \mathrm{~N}_{4}$-air interface, and the air-polysilicon interface. At each boundary, part of the optical power of the beam will be reflected and part will be transmitted. As the beam undergoes multiple reflections, an interference pattern will be created in the light collected back in the waveguide. These interference effects will change as the optical path length changes as the linear resonator stage moves to the left or right. It is this Fabry-Perot interference effect that will create a strong signal difference with small displacement, and subsequently be able to detect very fine motion.

To determine the resolution of the reflected interference signal, a first-order derivation will be performed, where only the initial reflected beams will be considered and multiple reflections are ignored.

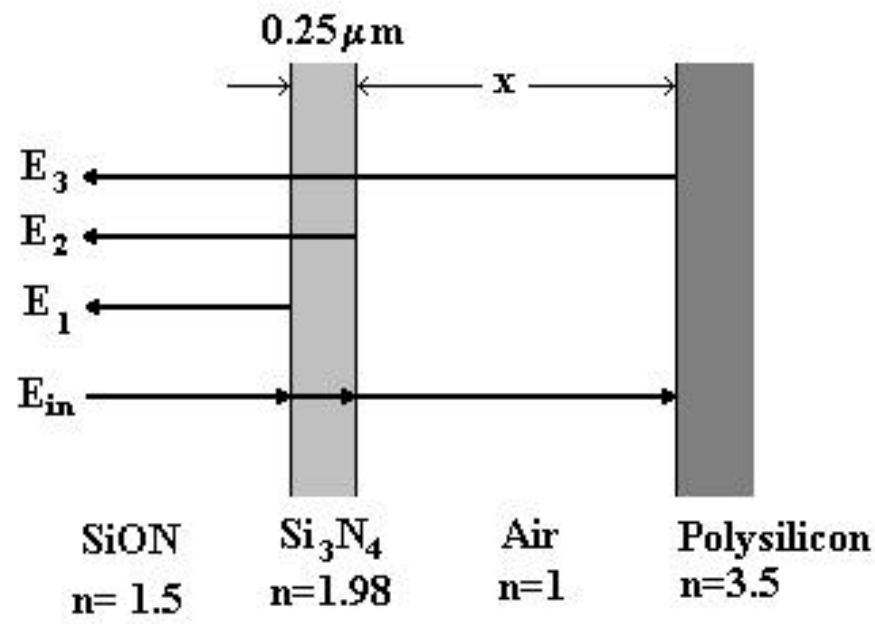

Figure 6-14: Endface Structure of Optical Waveguide. 
This analysis will be sufficient to determine the spacing in the interference pattern which will determine measurement resolution.

In Figure 6-14, it can be seen that three different optical signals are reflected back into the optical waveguide. By summing up these three signals, a graph of the output signal, electrical field potential can be generated. The input signal will take the form,

$$
E_{i n}=E_{0} e^{-j \omega t}
$$

$[35,59]$. The first optical field, $\mathrm{E}_{1}$, is the reflection of the input beam off the boundary between the silicon oxynitride and the silicon nitride. This surface has a reflectivity of 0.138 . Because the nitride has a higher index of refraction than the oxynitride, the reflected wave will undergo a phase shift of $\mathrm{p}$. Therefore the reflected electrical field is,

$$
E_{1}=0.138 E_{0} e^{-j(\omega t+\pi)}
$$

The second reflected optical field is created by the reflection of the input beam off the boundary between the silicon oxynitride and the air. This boundary has a reflectivity of 0.329 , but the beam has already penetrated the oxynitride/nitride boundary, which has a transmisivity of 0.86 . The reflected beam will then cross this boundary again. Therefore the magnitude of the reflected signal will be reduced by $(r)(t)^{2}$. Any, because the amount of light reflected off the surface more than once is negligible. The reflected optical beam travels a total path length of $0.5 \mathrm{Fm}$. The phase shift created by the optical path length difference can be calculated by multiplying the optical path length by the propagation constant $k$, which is equal to $n k_{0}$. The phase shift, $\mathrm{d}$, can be written by the equation,

$$
\delta=1.98 k_{0} 0.5 \mu m
$$


Using a wavelength of 1300 , the total phase shift is found to be 4.78 . Therefore the output electrical field, $\mathrm{E}_{2}$, can be written as,

$$
E_{2}=(0.329)(0.86)^{2} E_{0} e^{-j(\omega t+4.78)}
$$

The third reflected optical field is created by the reflection of the input beam off the boundary between the air and the polysilicon resonator stage. This boundary has a reflectivity of 0.556 , but the beam has already penetrated the oxynitride/nitride boundary and the nitride/air boundary, which has a transmisivity of 0.67 . The reflected beam will then cross both of these boundaries again, and the magnitude of the electric field will be reduced by $(\mathrm{r})\left(\mathrm{t}_{1}\right)^{2}\left(\mathrm{t}_{2}\right)^{2}$. The reflected optical beam travels a total path length of $2 x$, which is twice the length of the gap between the linear resonator and the waveguide endface. The phase shift $\mathrm{d}$, can be written by the equation,

$$
\delta=4.78+2 k_{0} x
$$

The signal also undergoes a phase shift of $\mathrm{p}$, upon reflection from the polysilicon, because it has an index of refraction higher than air. The output electrical field, $\mathrm{E}_{3}$, can be written as,

$$
E_{3}=(0.556)(0.67)^{2}(0.86)^{2} E_{0} e^{-j\left(\omega t+4.78+2 k_{0} x+\pi\right)}
$$

The total reflected optical field is the summation of these three fields:

$$
E_{t o t}=E_{1}+E_{2}+E_{3}
$$

[59]. This equation can be normalized by dividing it by the input electrical field, resulting in the equation,

$$
E_{\text {tot }}=0.138 e^{-j \pi}+0.243 e^{-j 4.78}+0.185 e^{-j\left(4.78+2 k_{0} x+\pi\right)} .
$$


The real part of the output field can be written,

$$
\begin{aligned}
& \operatorname{Re}\left\{E_{t o t}\right\}=0.138 \cos (-\pi)+ \\
& 0.243 \cos (-4.78)+0.185 \cos \left(-4.78-2 k_{0} x-\pi\right)
\end{aligned}
$$

Equation 6.9 is magnitude of the electrical field. The intensity, $\mathrm{I}_{\text {tot }}$, is equal to

$$
I_{t o t}=E_{t o t} E_{t o t}^{*}
$$

The intensity is graphed in Figure 6-15. The calculation was performed for $\mathrm{x}$ equal to $\pm 20 \mathrm{Fm}$.

Figure 6-16 shows a close up of the graph in Figure 6-15. In this graph, it can be seen that the period between optical maxima, which is greater than $0.7 \mathrm{Fm}$. Using a single mode waveguide probe can therefore detect motion of the linear stage on the order of $0.8 \mathrm{Fm}$.

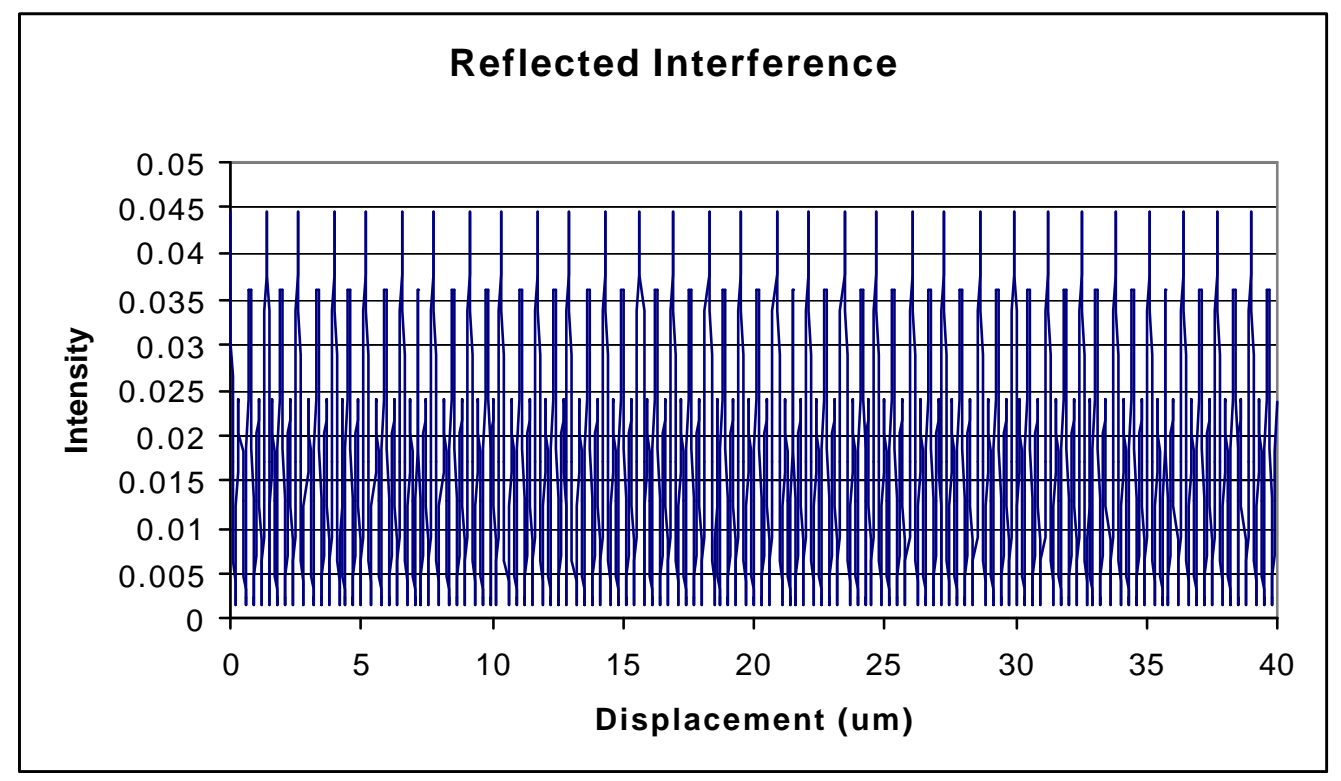

Figure 6-15: Interference Pattern Created by Reflected Optical Signal 


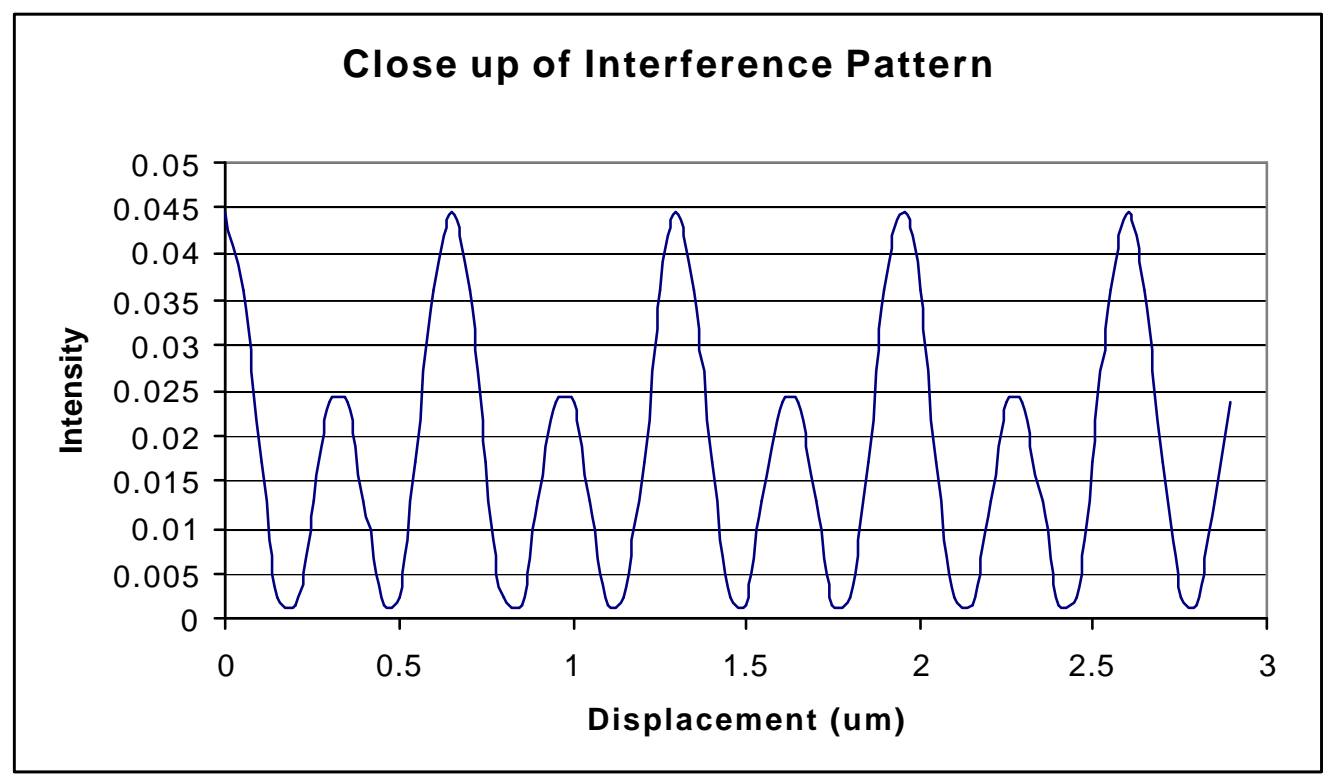

Figure 6-16: Reflected Signal Interference Pattern.

\subsection{Single Mode Integrated Optical Metrology}

Using inorganic optical waveguides to fabricate the integrated optical metrology structure

requires adding additional process steps to the MEMS fabrication process. Compared to the fabrication process for polymer multimode waveguides, the inorganic waveguide fabrication is more complex; however, the inorganic material processing steps are compatible with surface micromachinig processes. The advantage of the inorganic waveguides over the organic ones, is the ability to fabricate single mode waveguide structures. Single mode waveguides have the potential of sub-micron motion detection, due to the interference effects created by the reflected waves. 


\section{Chapter 7}

\section{Multimode Polymer Waveguides}

The co-integration of inorganic waveguides with MEMS structures requires adjusting the surface micro-machining process to accommodate the waveguide processing. However, using polymers to cointegrate waveguides with pre-fabricated linear comb resonators would be done in a post processing scenario which in combination with the foundry services available to researchers could have a significant impact on the technology without requiring extensive fabrication resources on the part of the researcher. In the case of the current program, linear resonator design can be fabricated at MCNC using the MUMPS process. Polymer waveguides can be fabricated in the lab at WVU upon the unreleased die. A cross section of the polymer waveguide co-integration is shown in Figure 7-1.
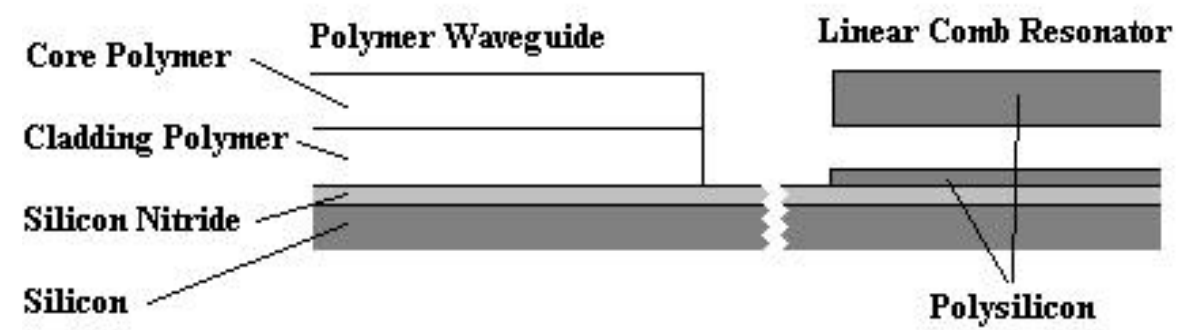

Figure 7-1: Cross Section of Organic Waveguide With a Fully Released Linear Comb Resonator. 
Because the MEMS devices are already fabricated, additional processing is a post-process, which is compatible with polymer material fabrication. When using optical polymers to fabricate waveguides it is presumed that they will be multimode, because most polymer indices are slightly higher than that of silicon dioxide, and because the polymer films of waveguide structures formed are relatively thick. The fabrication of single mode polymer waveguides, while achievable, is not considered here.

\subsection{Desired Optical Polymer Characteristics for the Formation of Co-}

\section{Integrated Optical Waveguides and MEMS Devices}

Previous discussion has presented the theoretical operation of the integrated optical metrology

probe. This research will also model and explore the feasibility of fabrication of the waveguide probe structure with pre-fabricated, linear resonator MEMS devices given the anticipated process requirements for polymer waveguides are within the scope of current microfabrication capabilities at WVU. Step one is identifying the right polymer to use, and finding a polymer is a process of matching application criteria.

An important polymer characteristic is how the application and patterning methods of the waveguides will be achieved. Spin casting of the polymer is required to fabricate the waveguide structure in Figure 7-1. The polymer must adhere to silicon nitride or silicon dioxide, since the sacrificial oxide will be removed before the application of the waveguides. In the lab at WVU, the simplest method of patterning of the waveguides is photo-patterning. This type of polymer patterning is desirable since both the cladding layer and the waveguide layer must be patterned. 
Post processing of the waveguides, after they have been fabricated requires that the polymer must be HF resistant, since they, as well as the MEMS devices will be submerged in a straight (50\% concentration) HF bath for 2.5 minutes. After the HF bath and rinse the waveguides will be subjected to a soak in isopropyl alcohol to reduce the probability of stiction. So it is also desirable that the fully cured film be resistant to alcohol [12]. The final baking temperatures will be $100 \mathrm{EC}$, therefore the glass transition temperature of the polymer must be above 100EC.

\subsection{Ultradel 9000 Polymers}

Final selection of the polymer material was the Ultradel 9000 series of polymers, which is manufactured by AMOCO [57]. The primary reason this polymer was chosen was because it was the only commercially available optical polymer in development quantities at the time this project began and throughout its duration, which fills the requirements listed previously.

The Ultradel polymer is synthesized in two formulations which act as a cladding and waveguide core. These flourinated polyimides have reasonable process characteristics and excellent thermal and optical stability. Both polymers are photo-patternable with UV light $(?=365 \mathrm{~nm})$. Films are subsequently developed using a special developer solution which is supplied with the polymers. This developer solution is Ether based, requiring extra-ordinary protection (an organic solvent filtered gas mask) when handling. The polymer materials have a glass transition of 390EC which is well above the desired temperature.

Optically the material has a transparency in the range of $633 \mathrm{~nm}$ to $1550 \mathrm{~nm}$. At a wavelength of $633 \mathrm{~nm}$ the material has an absorption coefficient of $0.32 \mathrm{~cm}_{-1}$, and at a wavelength of $1300 \mathrm{~nm}$ the 
material has an absorption coefficient $\mathrm{f} 0.08 \mathrm{~cm}_{-1}$. The cladding polymer has an index of refraction of 1.5479 at $633 \mathrm{~nm}$ and an index of 1.531 at $1300 \mathrm{~nm}$. The waveguide core polymer has a slightly higher index of 1.562 at a wavelength of $633 \mathrm{~nm}$ and an index of 1.544 at a wavelength of $1300 \mathrm{~nm}$ [57].

\subsection{Waveguide Cross-Sectional Structure}

The polymers will be spun-on the MEMS die, photo-patterned, and developed. The two easiest waveguide structures to fabricate are strip waveguides and buried strip waveguides. These are shown in Figure 7-2. The strip waveguide is created using a two layer process; first a cladding is applied and patterned, then the waveguide core is applied and patterned. In this case, the core is clad on the bottom by polymer and clad by air on the remaining three sides. The embedded strip waveguide is a three layer process, because a top coat of cladding polymer is applied so that the waveguide core is completely surrounded by cladding polymer.

The main difference between these two waveguides is the difference in number of modes that the same size core will support. Because air has a refractive index of 1 , the index difference between the
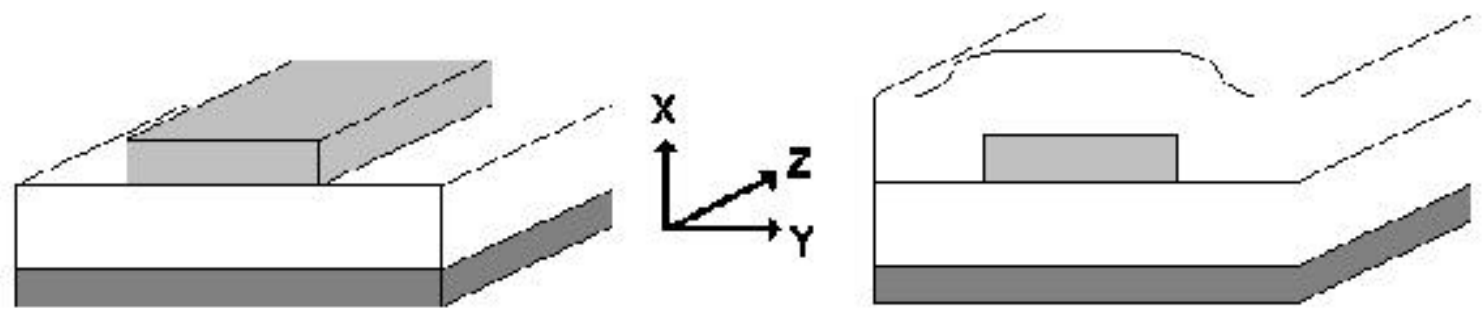

Figure 7-2: a) left is the strip waveguide and b) right is the embedded strip waveguide 
core and air is much greater than that for the difference between the core and cladding polymer. A larger index of refraction difference between two materials increases the critical angle (which dictates total internal reflection) and therefore increases the number of modes supported in the guide. Therefore a waveguide core that is bounded on both sides by air will support more modes than a waveguide core of the same size bounded by cladding polymer.

\subsection{2-D Modal Simulations}

One piece of information that will be needed when designing waveguide layouts is the physical dimensions of the guide. It is important to know what thicknesses and widths of the guide will create single mode guides and multimode guides. To determine guide thickness cutoff between single mode and multimode, the optical waveguide simulation tool, BPM_CAD by Optiwave will be used. The part of the modeling tool used for mode solving is the 2-D Mode Analysis. This portion of the software determines the number of modes that can be supported in a 2-D or slab dielectric waveguide using the effective index method. In this modeling approach a 2-D waveguide cross section is decomposed into two 1-D guides along its symmetry axis [58].

Looking back in Figure 7-2, there are three possible effective 1-D cross sections that can occur in these two different waveguide structures. In the strip waveguide of Figure 7-2a, the waveguide core is clad on both sides by air in the x-direction. In the y-direction, the waveguide core is clad on top by air and on the bottom by cladding polymer. The embedded strip waveguide of Figure $7-2 b$ is clad on both sides by the cladding polymer in both the $\mathrm{x}$ - and $\mathrm{y}$-directions. Therefore these three cladding profiles respectively: air-guide-air, air-guide-cladding, and cladding-guide-cladding, will be modeled to 
solve for mode profiles. This effective index treatment assumes fields do not appreciably extend beyond the boundaries of the end regions and is most accurate for modes far from cut off.

The thicknesses of the waveguide core is varied to determine the largest thickness of the waveguide core that will still support a single-mode. In the following simulations, the mode structures are modeled at two different wavelengths, and for each simulation set, different indices of refraction were used. At a wavelength of $632.8 \mathrm{~nm}, \mathrm{n}_{\text {air }}=1, \mathrm{n}_{\text {guide }}=1.562$, and $\mathrm{n}_{\text {cladding }}=1.5479$. At a wavelength of $1300 \mathrm{~nm}, \mathrm{n}_{\text {air }}=1, \mathrm{n}_{\text {guide }}=1.544$, and $\mathrm{n}_{\text {cladding }}=1.531$. The waveguide core thickness was varied in each simulation to find the maximum dimension that the core could be and still support a single mode [57].

\section{Air-Guide-Air Model}

The first simulation was to create an air-guide-air slab dielectric was to simulate the cross section of the strip waveguide in the y-direction. The following figures: Figure 7-3 and Figure 7-4, display the mode profiles at the $632.8 \mathrm{~nm}$ and $1064 \mathrm{~nm}$ wavelengths respectively. These figures are the mode profiles at the cut off thicknesses for single mode guiding. At a wavelength of $632.8 \mathrm{~nm}$ the thickness was found to be $0.26 \mathrm{Fm}$, and at a wavelength of $1300 \mathrm{~nm}$, the thickness was found to be $0.55 \mathrm{Fm}$.

\section{Air-Guide-Cladding Model}

The second slab dielectric waveguide that was simulated was the air-guide-cladding model. This simulation was to replicate the x-direction cross-section of the strip waveguide, where the waveguide core is clad by air on the top and clad by polymer on the bottom. Figure 7-5 shows the mode profile in a $2.21 \mathrm{Fm}$ thick waveguide at a wavelength of $632.8 \mathrm{~nm}$. This is the 


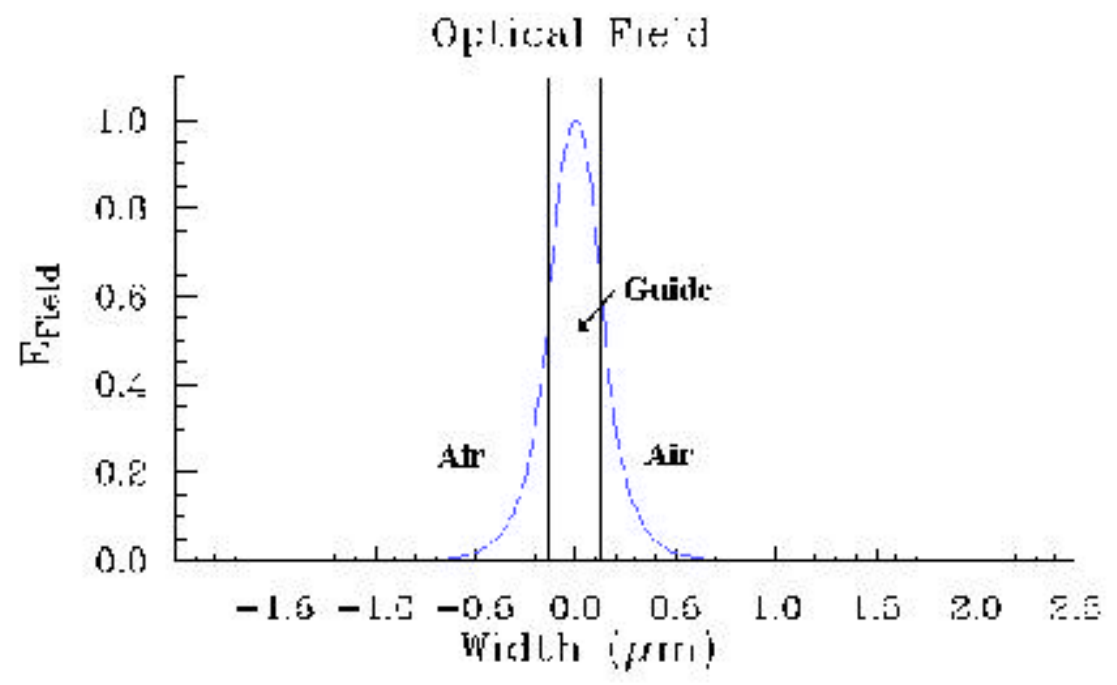

Figure 7-3: Single-Mode Profile at a Wavelength of $632.8 \mathrm{~nm}$.

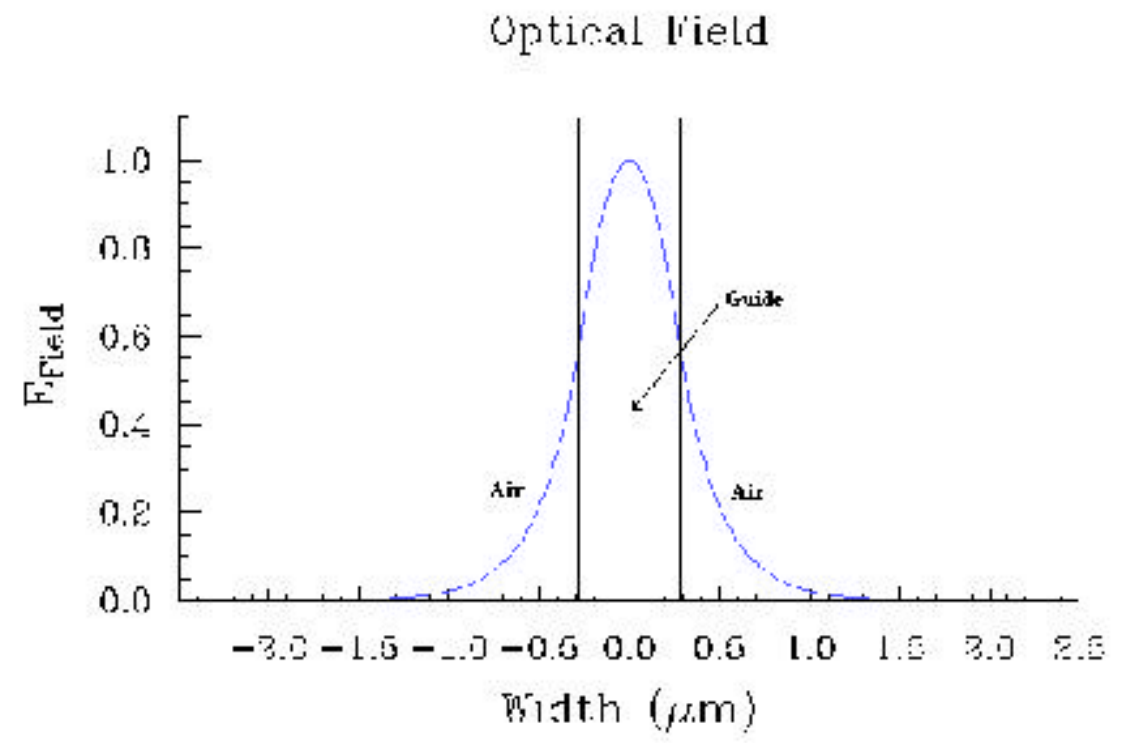

Figure 7-4: Single-Mode Profile at a Wavelength of $1300 \mathrm{~nm}$. 


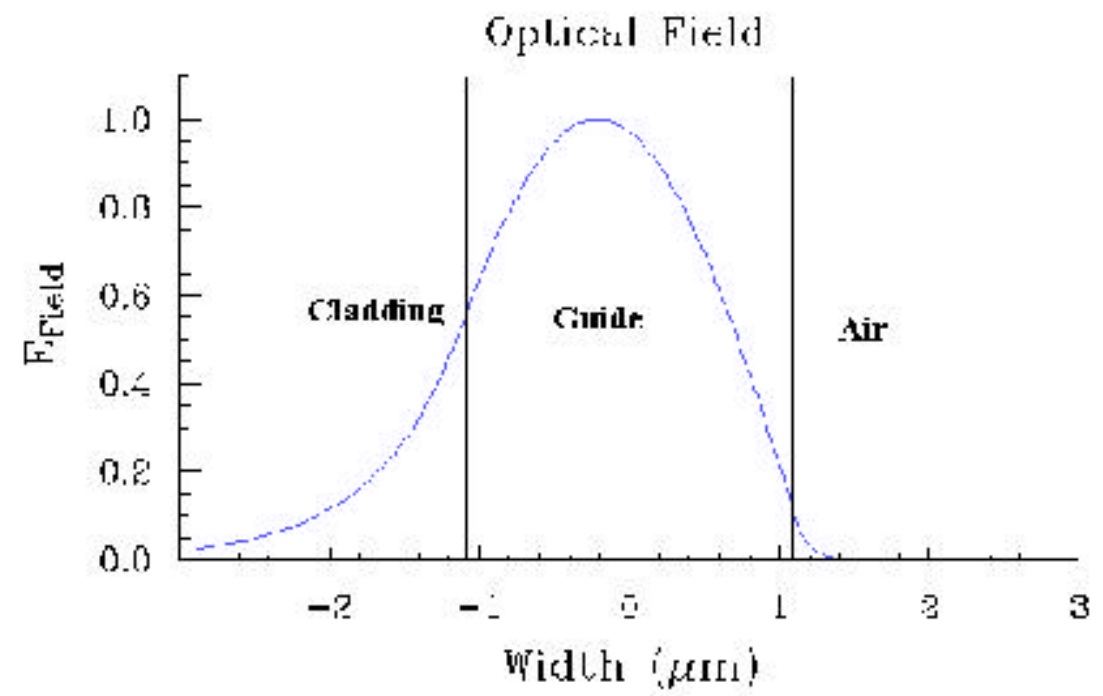

Figure 7-5: Single-Mode Profile at a Wavelength of $632.8 \mathrm{~nm}$.

Optinal liield

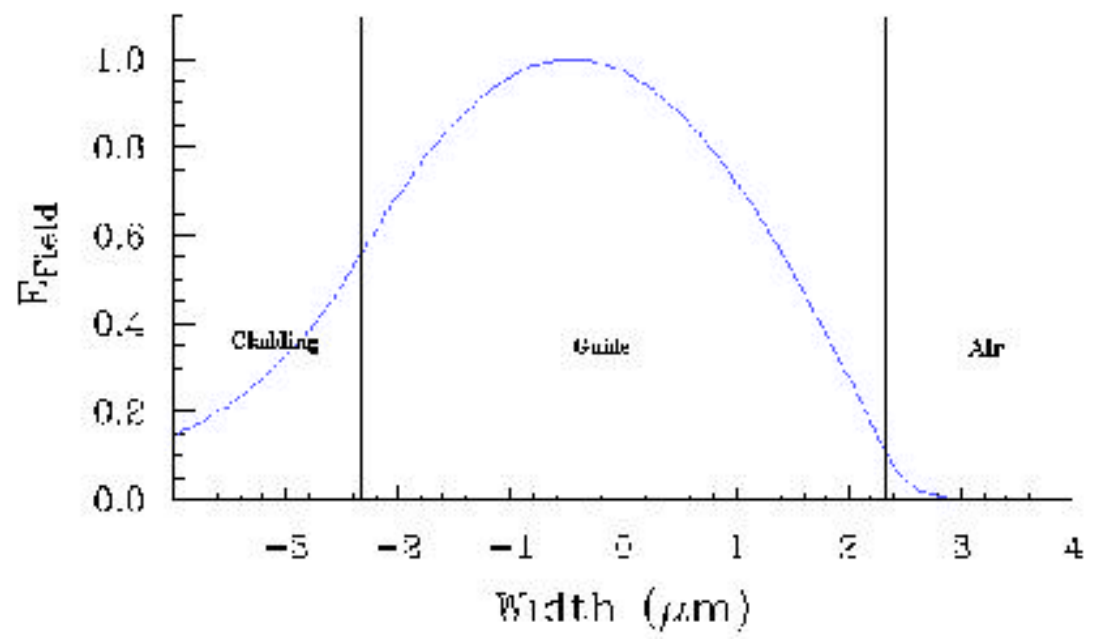

Figure 7-6: Single-Mode Profile at a Wavelength of $1300 \mathrm{~nm}$. 
thickest guide that will support only one mode. Figure 7-6 shows the mode profile at a wavelength of $1300 \mathrm{~nm}$, where the cut-off thickness was found to be $4.73 \mathrm{Fm}$.

\section{Cladding-Guide-Cladding Model}

The final simulation set was performed using the cladding-guide-cladding model. This simulation can be used to represent both the x-axis and the y-axis cross-section of the embedded strip waveguide, where the core is completely surrounded by polymer. In these simulations, the maximum thickness of a single mode guide at the $632.8 \mathrm{~nm}$ is $1.57 \mathrm{Fm}$. The profile of this guide is shown in Figure 7-7. The other simulation showed that at a wavelength of $1300 \mathrm{~nm}$ the cut-off thickness is $3.36 \mathrm{Fm}$. The profile for this waveguide is shown in Figure 7-8.

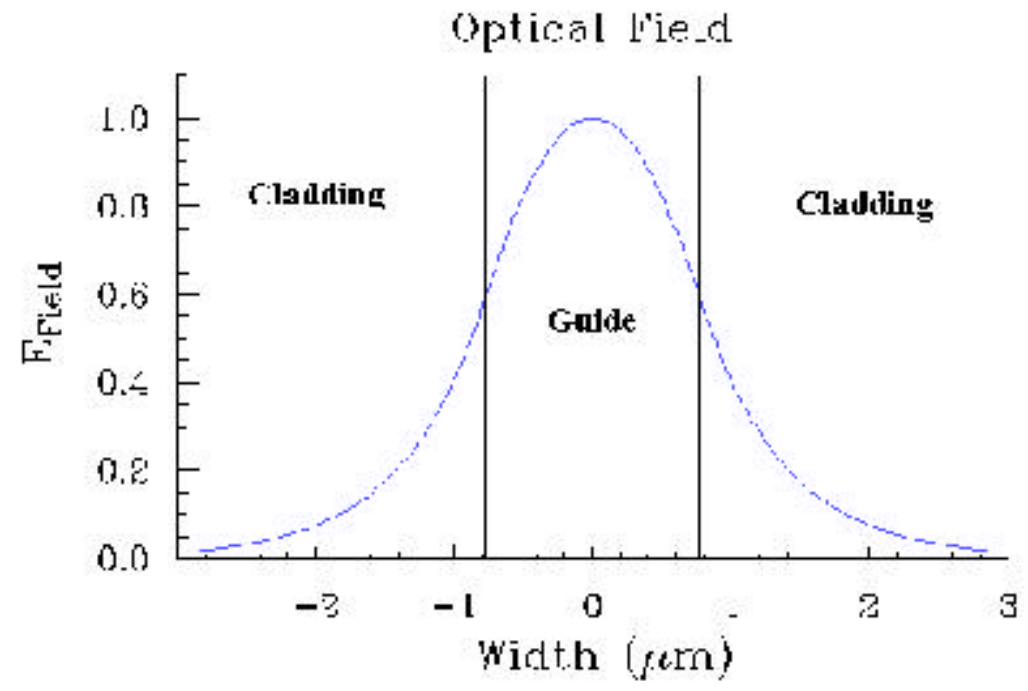

Figure 7-7: Single-Mode Profile at a Wavelength of 632.8 Fm. 


\section{Optinal liield}

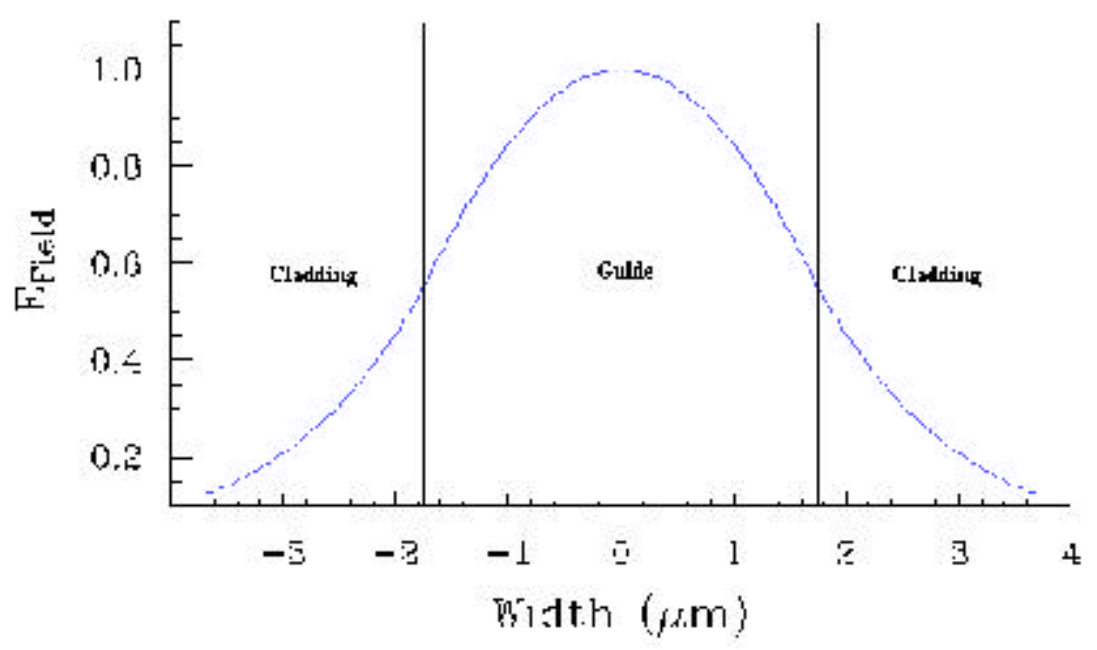

Figure 7-8: Single-Mode Profile at Wavelength of $1300 \mathrm{~nm}$.

\subsection{Single Mode Analysis}

The goal of finding the maximum thickness that the waveguide core could be and remain single mode was found to determine the minimum allowable thickness for a multimode guide. Table 7-1 summarize the minimum dimensions discovered using the 2-D mode profile solver. In this table the top two rows represent the minimum width and thickness of a strip waveguide structure respectively. The third row represents both the minimum thickness and width for an embedded strip waveguide.

The thinnest Ultradel film that can be applied is $2 \mathrm{Fm}$, and the thinnest line that can be readily patterned using the current WVU aligner facilities is $3 \mathrm{Fm}$. So the minimum dimensions of a waveguide core is $2 \mathrm{Fm} \times 3 \mathrm{Fm}$. These dimensions automatically discount creating single mode waveguides, either both strip or embedded strip, for a wavelength of $632.8 \mathrm{~nm}$. However 
the embedded strip waveguide can be used as a single mode waveguide at a wavelength of $1300 \mathrm{~nm}$ or greater.

\begin{tabular}{|l|r|r|}
\hline \multicolumn{3}{|c|}{ Minimum allowable thickness for a multimode guide. } \\
\hline Structure & $@ 632.8 \mathrm{~nm}$ & $@ 1300 \mathrm{~nm}$ \\
\hline Air-Guide-Air & $0.27 \mathrm{Fm}$ & $0.56 \mathrm{Fm}$ \\
\hline Air-Guide-Cladding & $2.22 \mathrm{Fm}$ & $4.74 \mathrm{Fm}$ \\
\hline Cladding-Guide-Cladding & $1.58 \mathrm{Fm}$ & $3.37 \mathrm{Fm}$ \\
\hline
\end{tabular}

Table 7-1: Single Mode Waveguide Thickness as a Function of Wavelength and Cladding Structure.

\subsection{Waveguide Layout}

Reviewing the above analysis and given the available fabrication facilities at the time, it was determined that multimode waveguiding structure would be the focus of the polymer work. When the waveguide mask was developed and the co-integration process laid-out, other factors also influenced the choice of multimode guides, over single mode guides based upon initial processing tests with the Ultradel polymer. As part of the patterning, the polymers are spray developed with a solvent, during this process the structures absorb some of the developer solution which causes the polymers to swell. Predominant swelling occurred on the edges and sidewalls. By creating large structures, the proportion of irregularities to waveguide features is minimized.

Another motivation for using multimode waveguides was the fact that larger endfaces make optical coupling alignment easier, enabling more optical power to be coupled to the guide and less severe alignment tolerances. In addition heavily moded guides are known to be less susceptible to 
processing imperfections and substrate topography. This is viewed as especially important in the highly topographic post processed surface micromachined environment.

A picture of the mask layout for the waveguides is shown in figure 7-9. The waveguide alignment with respect to the linear resonator devices is shown in Figure 7-10. As with the single mode guides discussed in the previous chapter, the waveguide probe consist of two legs. One leg is used to introduce light into the structure and the other leg is coupled to a detector.

The width of the waveguide probe integrated next to the resonator stage is $100 \mathrm{Fm}$ wide and the width of each leg is $50 \mathrm{Fm}$ wide. These structures utilize reflecting angle bends. They are utilized here because the mask layout software (LASI 5.0) available at the time could easily implement manhattan geometries, modified with straight line polynomials, but it was very difficult to draw arcs and curves.
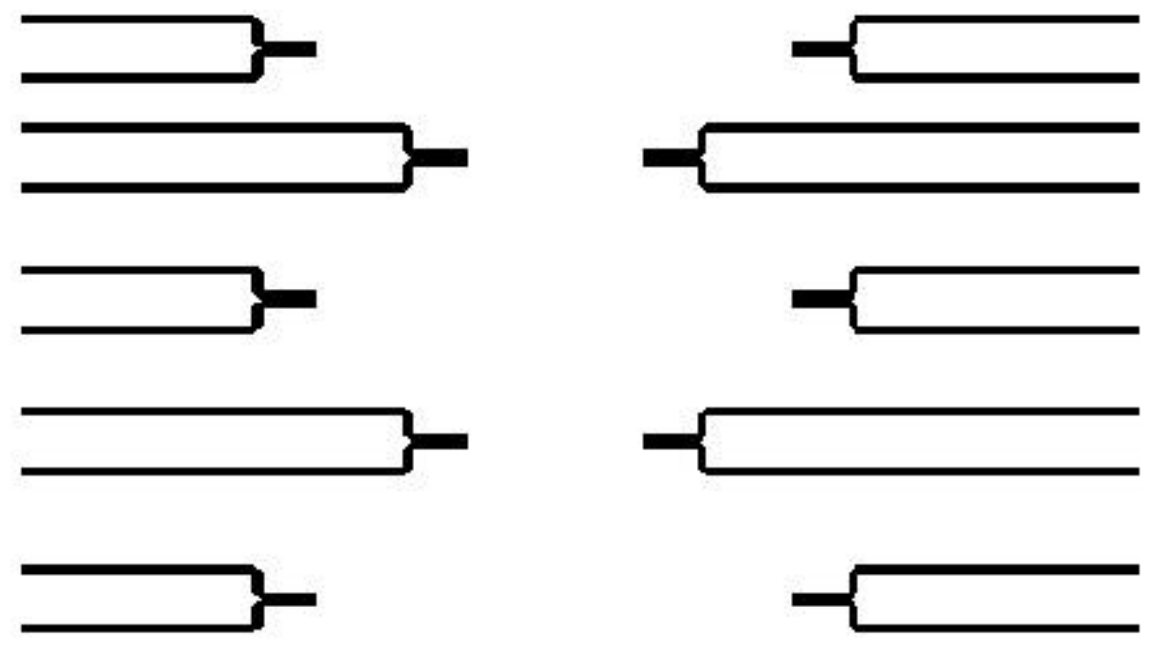

Figure 7-9: Mask Layout of Polymer Waveguides. 


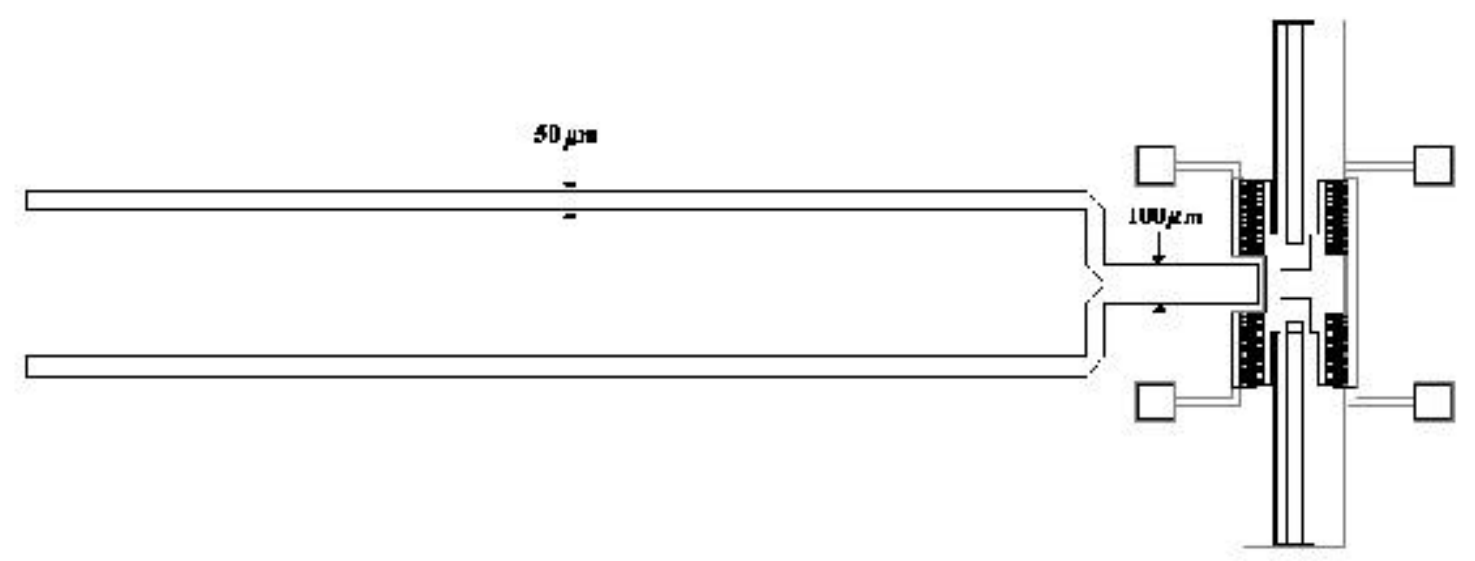

Figure 7-10: Multimode Waveguide Probe Structure Aligned with Linear Resonator.

The length of the $100 \mu \mathrm{m}$ segment of waveguide next to the linear comb resonator was chosen to be $400 \mu \mathrm{m}$. This was the minimum length necessary for this segment so that the probe legs do not overlap the contact pads of the resonator. In the following simulations, the length of this waveguide segment emerges as an important parameter when designing the waveguide probes. It is one of the parameters which will determine the amount of mode filling that occurs within this segment of waveguide.

\subsection{Simulated Optical Measurement of Linear Resonator Displacement}

The large dimensions for the multimode waveguide structure were chosen to alleviate alignment difficulties during fabrication and maximize input power coupling. However, this represents a tradeoff with ideal IOM performance. While the strip waveguide structure will only support one mode along the 
$\mathrm{x}$-axis, the $50 \mathrm{Fm}$ wide waveguide legs in the structure in Figure 7-10 will support 189 modes along the y-axis. Both legs of the waveguide structure are extremely multimode, while coupling into the large waveguides has been made easier, in practice it will be difficult to fill all the modes in the waveguides. As a result these large waveguides effectively act as slab dielectric waveguides, with clearly evident total internal reflection of the modes inside the guide which are filled. This is demonstrated in Figure 7-11, which is the 2-D propagation simulation of a $100 \mathrm{Fm}$ waveguide.

The waveguide simulation in Figure 7-11 shows an example where only part of the "middle" modes are filled and the light totally internal reflects inside the waveguide. This waveguide has taken on properties similar to a slab dielectric waveguide. Both the $50 \mathrm{Fm}$ and the $100 \mathrm{Fm}$ segments will only be partially filled. The waveguide structure in Figure 7-10 creates two optical paths: the input path which carries optical power to illuminate the linear resonator stage and the return optical path, which collects

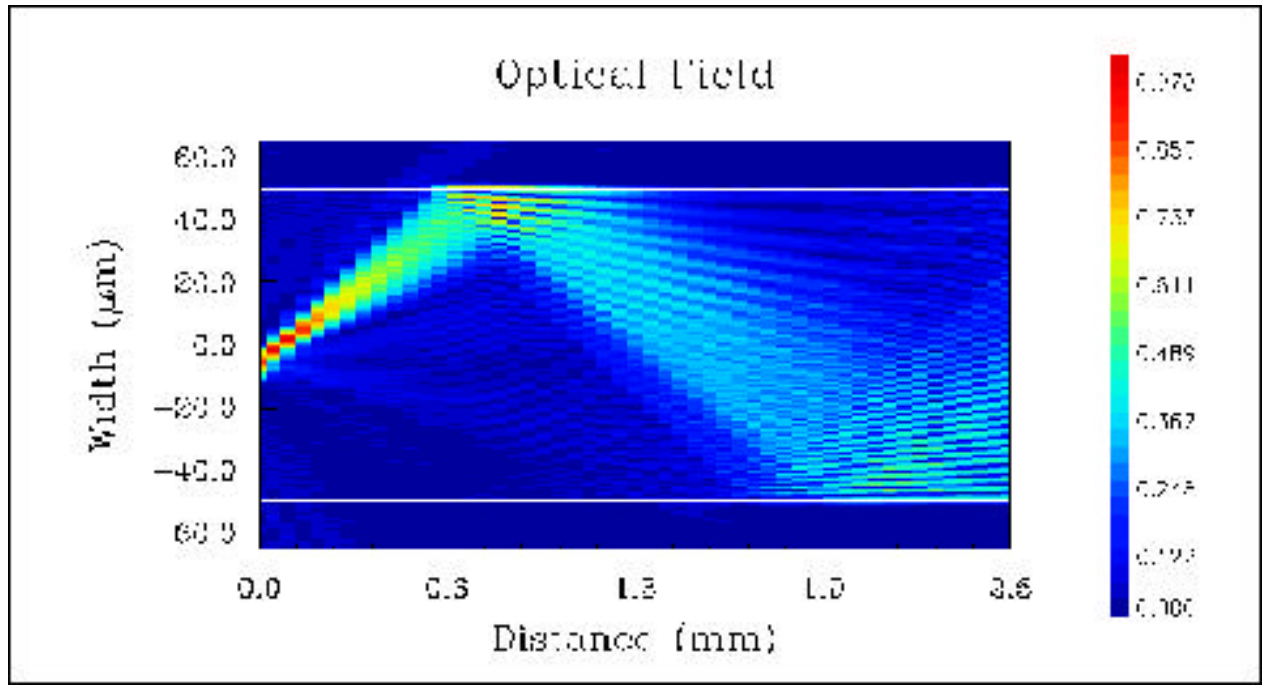

Figure 7-11: Optical Propagation of Partially Filled Modes in 100 Fm Polymer Waveguide. 
the reflected light and carries the reflected optical power to the detector. As shown in Figure 7-12 (a), (b), and (c), the optical path that the light follows will be dependent upon mode order. Figure 7-12(a) shows the optical path of the primary mode, Figure 7-12(b) shows the optical path of a "middle: order mode, and Figure 7-12(c), shows the optical path of a high order mode. The optical path that the reflected light will follow is also dependent upon the angle of reflections inside the $100 \mathrm{Fm}$ segment of the waveguide structure. As shown in Figure 7-12 (a), the primary mode path is perpendicular to the linear resonator stage edge, which will reflect the light back along the same path and very little light will reach the detector.

As with the single mode waveguide structure, to simulate the reflection of a signal from the linear resonator stage, it is assumed that the stage edge is a perfectly reflecting structure, so that the waveguide could be mirrored. This is shown in Figure 7-13. Because BPM_CAD cannot simulate strong vertical components of the electric field, the program cannot simulate the reflected angle bends, which require the optical path to travel in the y-direction. Therefore for the simulations only the $400 \mathrm{Fm}$ segment of the waveguide was simulated, as shown in Figure 7-14. The injection of the modes was simulated by creating input fields which could be treated as input to the waveguide structure. Because the simulation tool cannot handle strong vertical elements, simulations are performed for partial filling of the primary modes and lower order modes only. Four different simulations were performed to represent the different angles. 


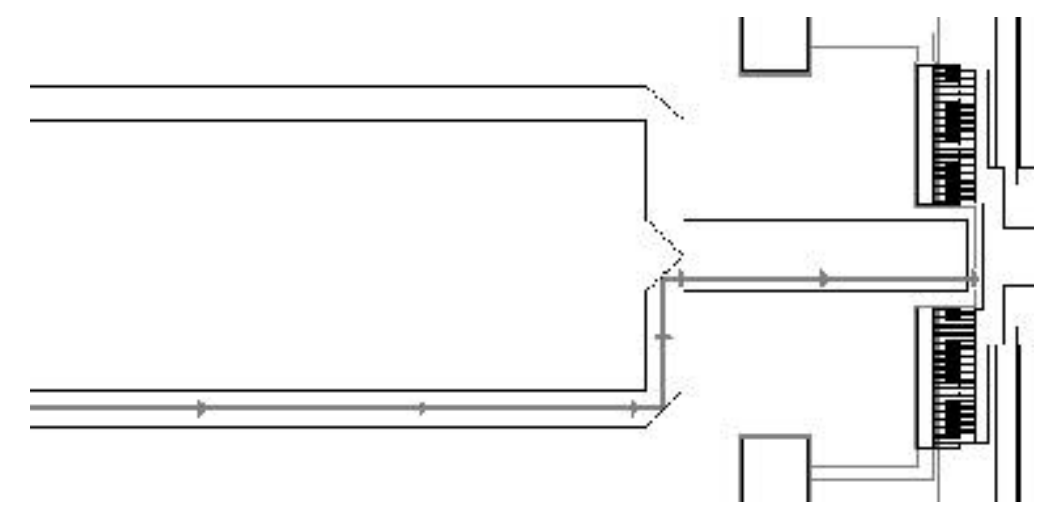

a) Primary Mode

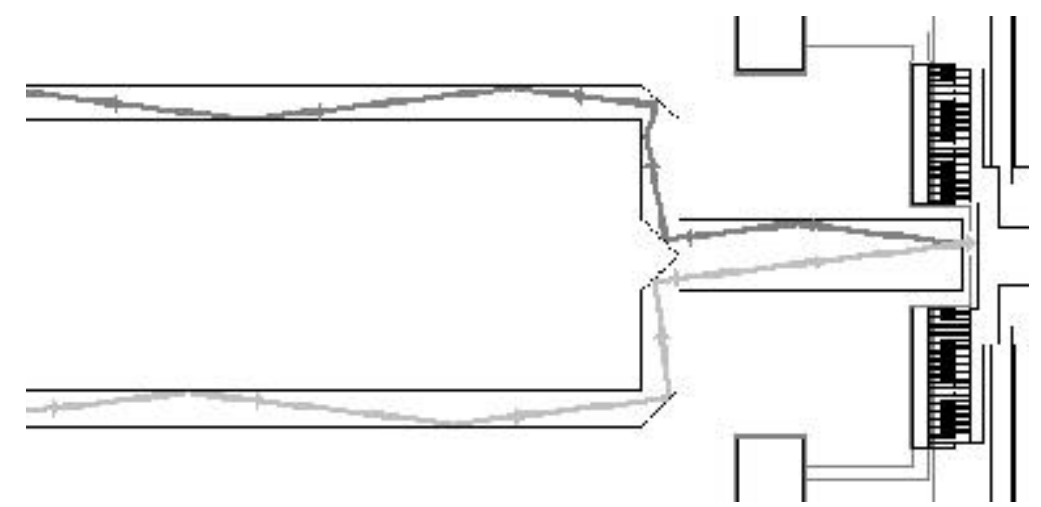

b) Middle Order Mode

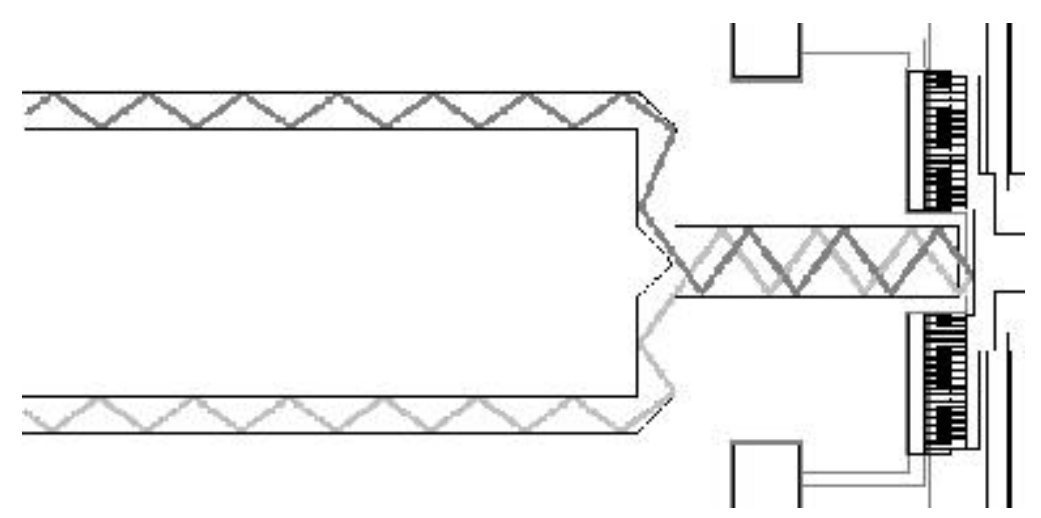

c) Higher Order Mode

Figure 7-12: Partially Filled Modes in Integrate Optical Metrology Structure. 


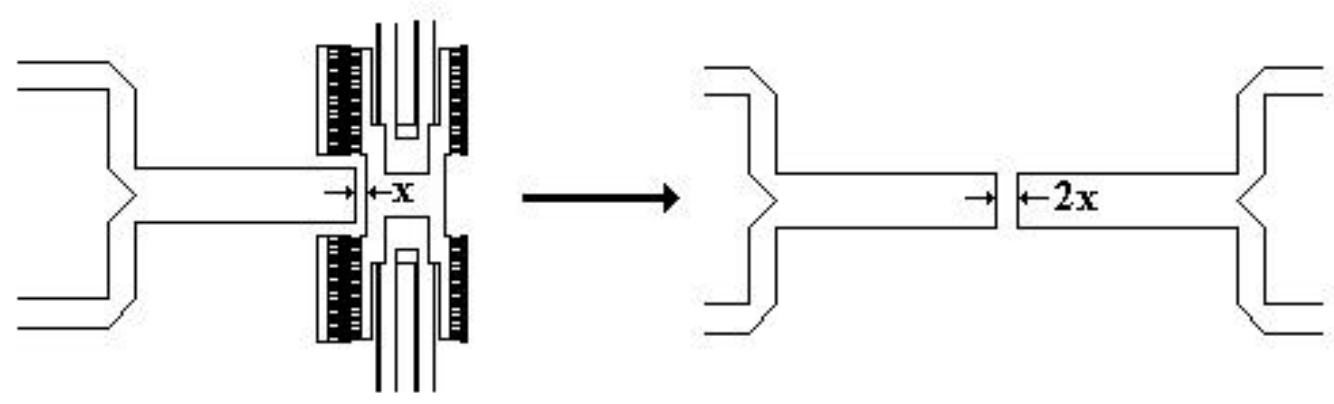

Figure 7-13: Reflection of Waveguide Structure to Simulate Optical Gap between Linear Resonator and Waveguide.

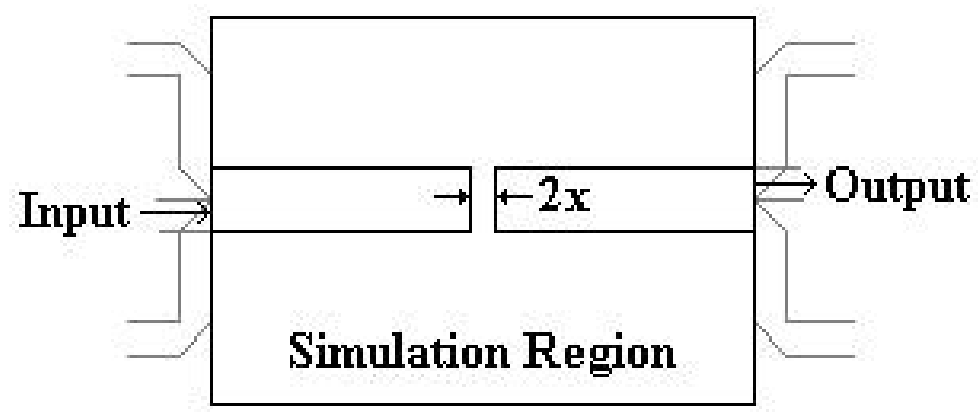

Figure 7-14: Region of Simulation of the Reflected Waveguide Structure.

The optical power out, divided by the input optical power was calculated for different gap sizes, which represent different deflection amounts of the linear resonator stage. Output optical signals were calculated for only the upper $50 \mathrm{Fm}$ of the waveguide to simulate the output optical path. 
Figure 7-15 depicts a distribution of the optical intensity inside the $400 \mathrm{Fm}$ waveguide segment; in this figure, the simulated gap is $20 \mathrm{Fm}$. This first simulation shows the partial filling of the waveguide which has most of the optical power contained in the lowest order modes. Figure 7-16 is a graph of the calculated output power ratio using BPM_CAD 2D propagation module. The amount of power in the reflected beam is around $20 \%$ and is maximum when the linear stage deflection is around $8 \mathrm{Fm}$ in the positive $\mathrm{x}$ direction (a deflection distance of $28 \mathrm{Fm}$ ).

Figure 7-17 also depicts a distribution of the optical intensity inside the $400 \mathrm{Fm}$ waveguide segment with a $20 \mathrm{Fm}$ gap. This simulation attempts to partially fill the waveguide modes which has the optical power contained in the lower order modes. Figure 7-18 is the graph of the calculated output power ratio. The amount of power in the reflected beam is around $30 \%$ and is maximum when the linear stage deflection is around $10 \mathrm{Fm}$ in the positive $\mathrm{x}$ direction (a deflection of $30 \mathrm{Fm}$ ).

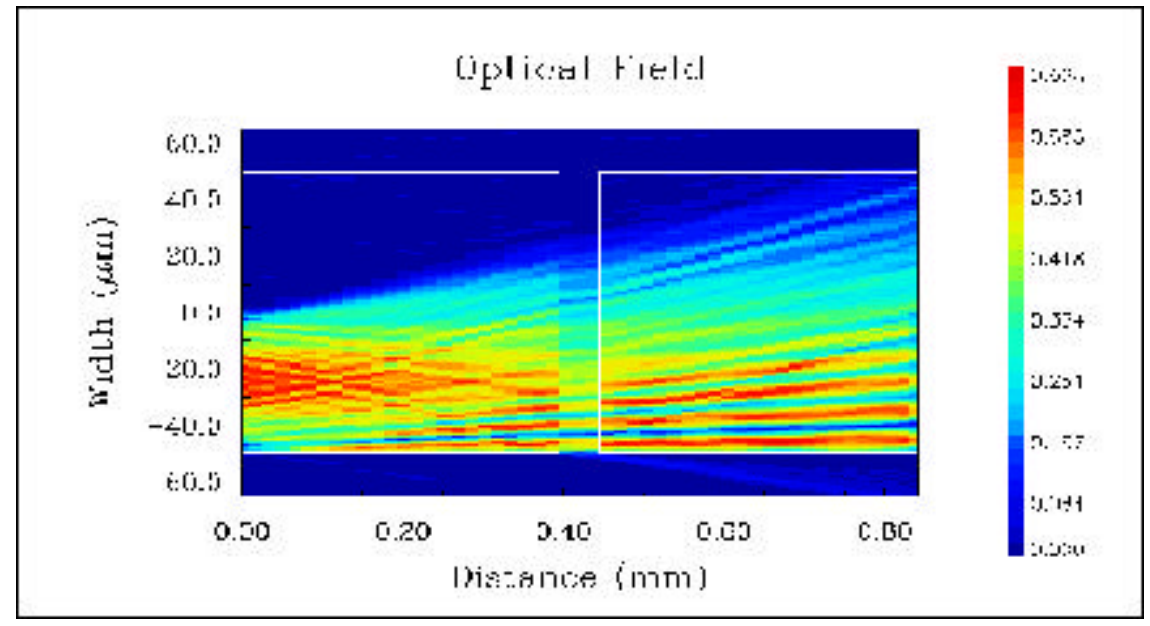

Figure 7-15: Optical Power Filling of Lowest Order Modes. 


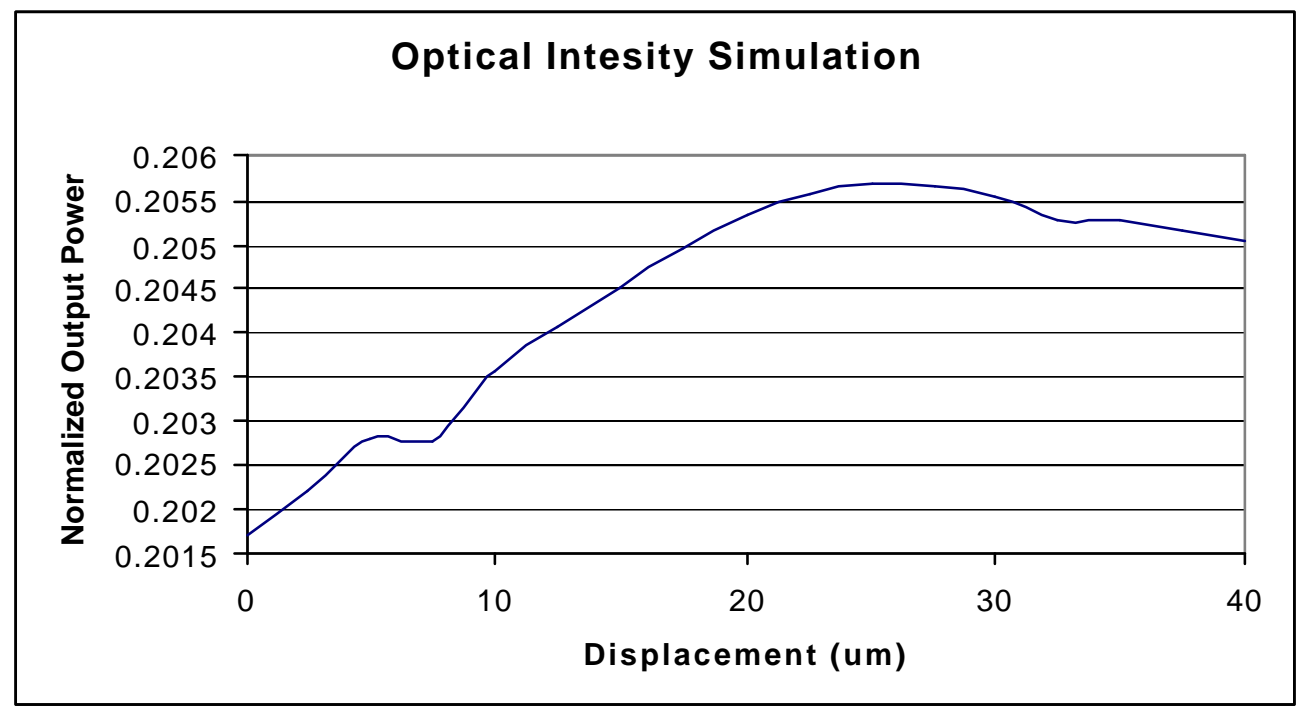

Figure 7-16: Simulated Output Signal Intensity with Lowest Order Modes Filled.

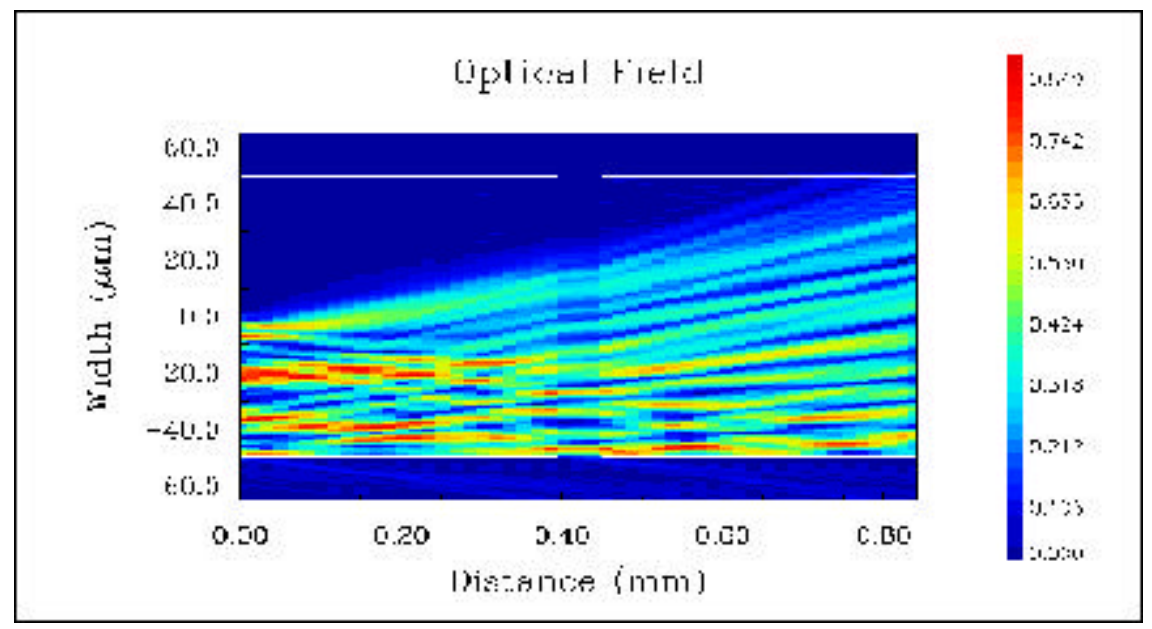

Figure 7-17: Optical Power Filling of Lower Order Modes. 


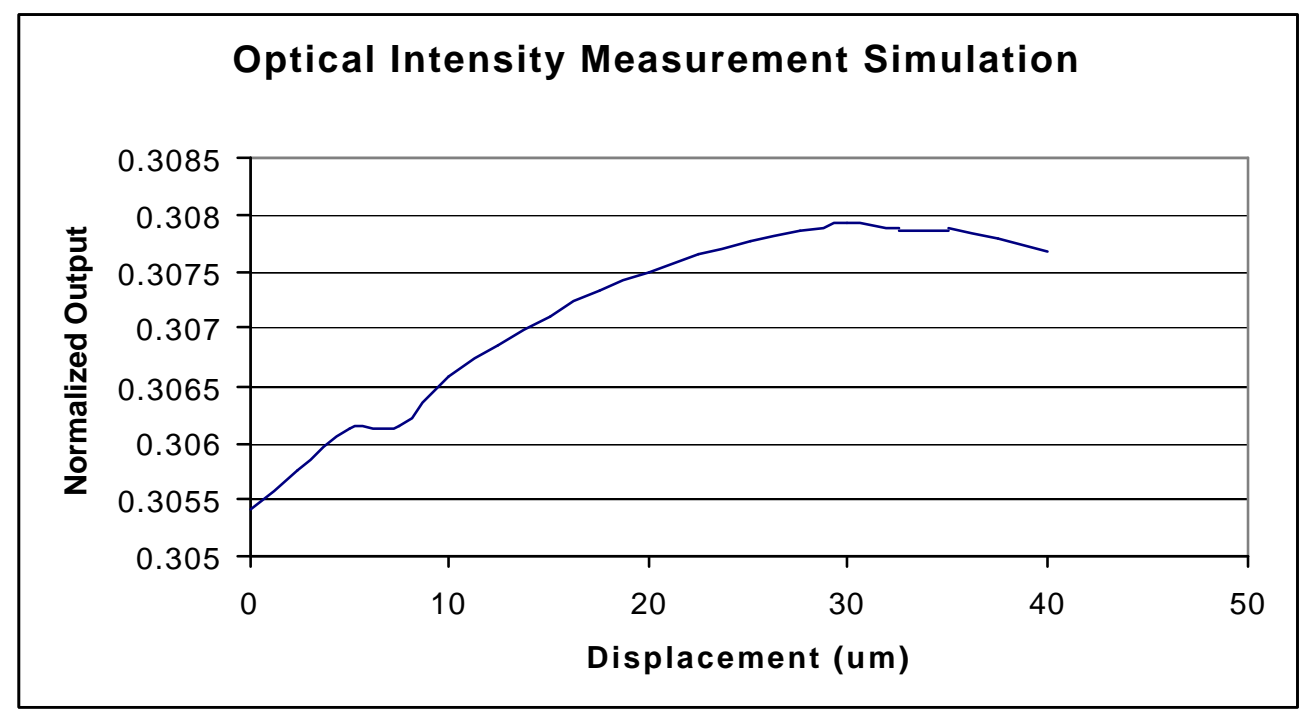

Figure 7-18: Simulated Output Signal Intensity with Lower Order Modes Filled.

Figure 7-19 displays the optical distribution in the $400 \mathrm{Fm}$ segment. In this simulation the deflection angle of the beam is higher and so more power is coupled into relatively higher order modes; however, the optical power coupling is still in the lower ordered modes. The graph of the measured feedback intensity is shown in Figure 7-20. In the graph in Figure 7-20, the detected optical signal is about $70 \%$ of the input signal and the value is around $15 \mathrm{Fm}$ (a deflection of $35 \mathrm{Fm}$ ). The intensity of the feedback signal is dependent upon which modes are filled in the waveguide probe structure due to the extremely large dimensions of the waveguide.

The intensity of the feedback signal is dependent upon which modes are filled in the waveguide probe structure. The problem with these measurements is the extremely large dimensions of the waveguide. 


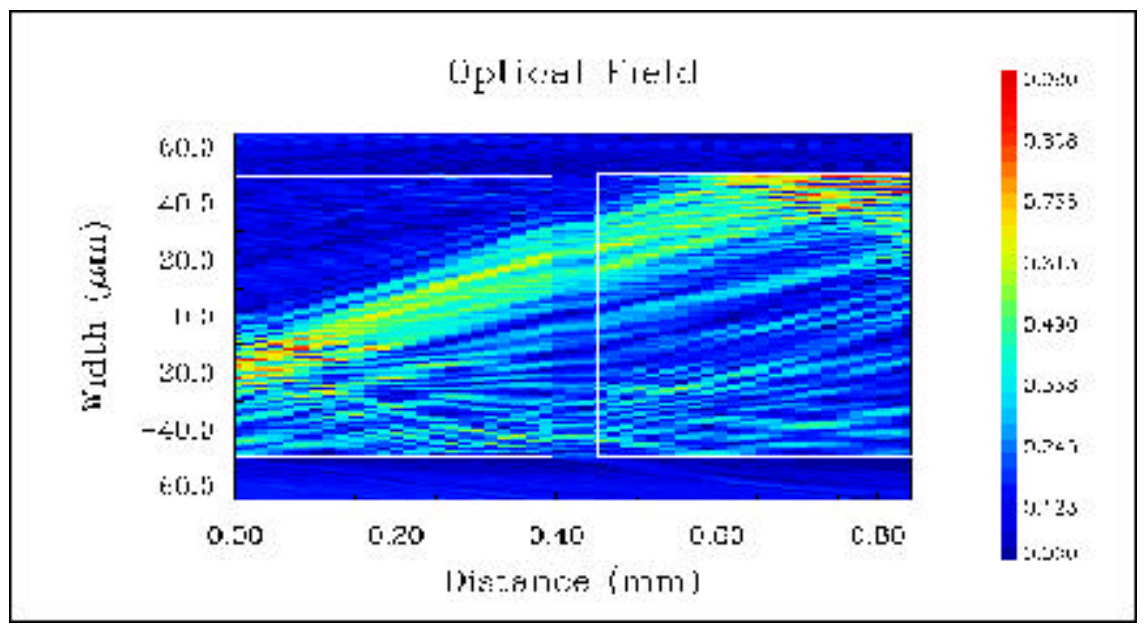

Figure 7-19: Optical Field Distribution of Relatively Higher Ordered Modes.

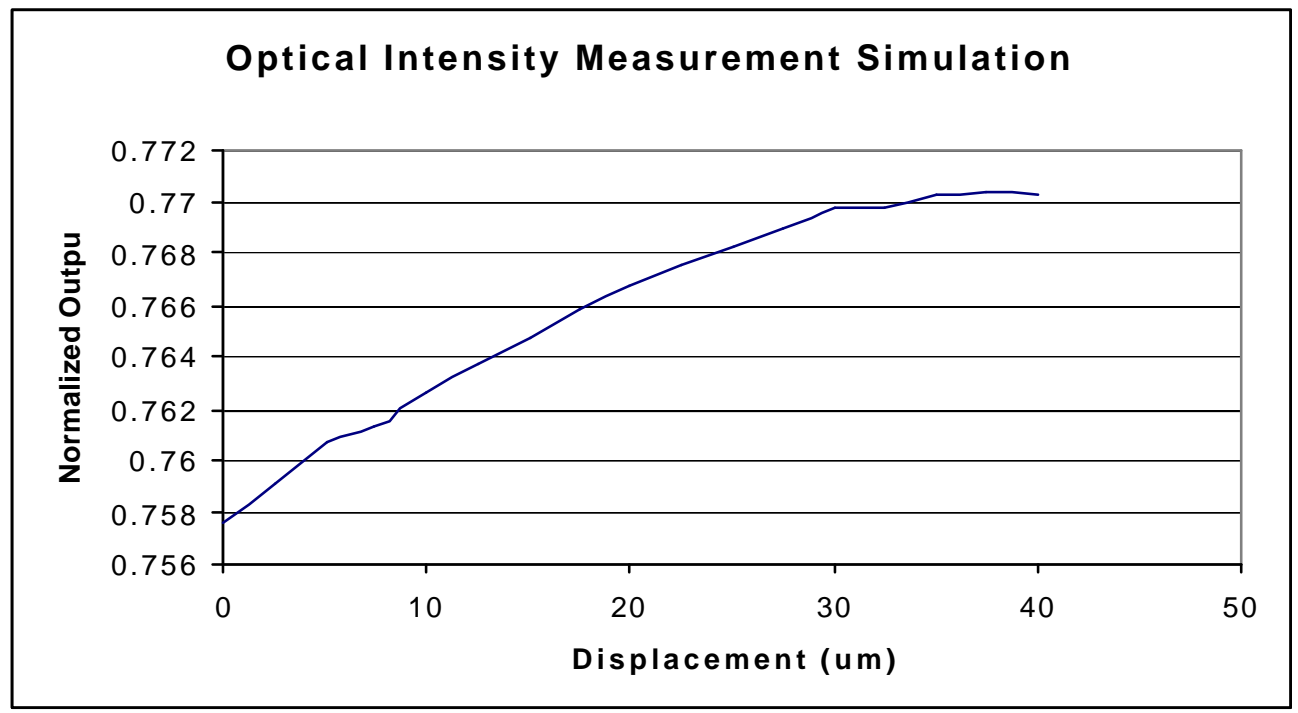

Figure 7-20: Simulated Optical Output Intensity with Relatively Higher Order Modes Filled. 
Another simulation was performed using a $12 \mathrm{Fm}$ waveguide. In this waveguide structure the legs of the guides would be $6 \mathrm{Fm}$ wide. A $6 \mathrm{Fm}$ waveguide of Ultradel polymers will support 22 modes and a $12 \mathrm{Fm}$ waveguide will support 45 . Even though there are fewer modes, and it is easier to couple the optical power into all of them, the $12 \mathrm{Fm}$ waveguide segment will not have all the modes filled, because the input waveguide is only $6 \mathrm{Fm}$. Figure 7-21 shows the optical power coupling in the $12 \mathrm{Fm}$ waveguide, the gap spacing if for a $20 \mathrm{Fm}$ gap between the waveguide and the linear resonator stage. A graph of the normalized optical output of these simulations is shown in Figure 7-22. In this graph the differences in optical output intensity is stronger, the output varies from $38 \%$ to $45 \%$ of the input power. While larger polymer waveguide structures may simplify alignments during the fabrication process, a smaller polymer waveguide will experience more optical loss over the deflection distance and therefore yield a greater change in output intensity. This advantage must be balanced against the increased difficulty in optical input coupling associated with these smaller waveguides, and thier increased susceptibility to dimensional variatons (e.g. due to process, topography, etc.) 


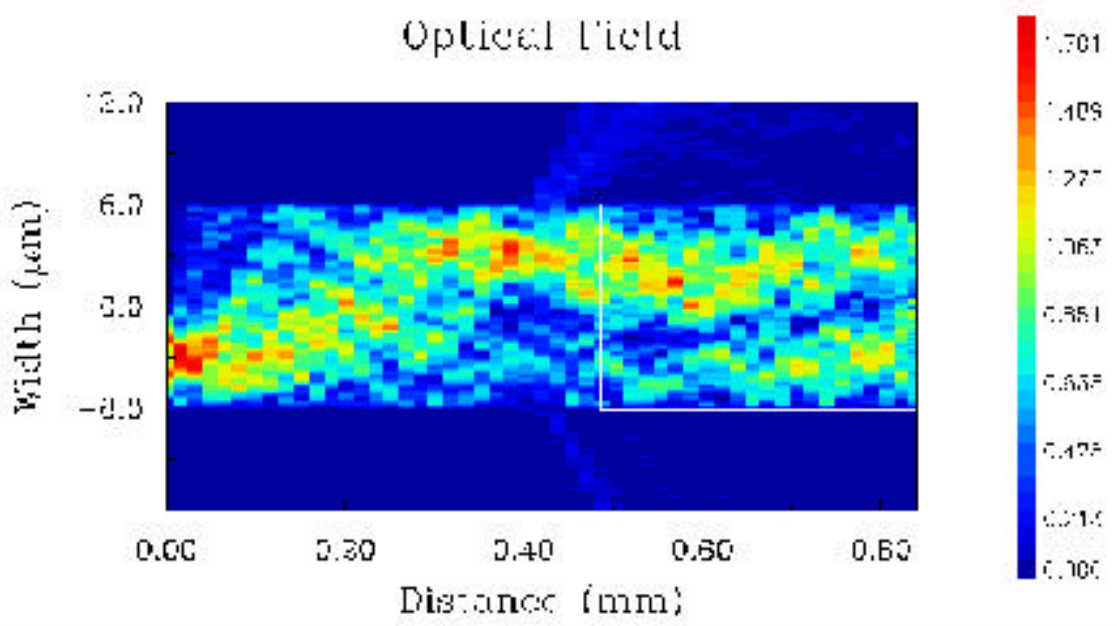

Figure 7-21: Optical Field Distribution in a $12 \mathrm{Fm}$ Waveguide.

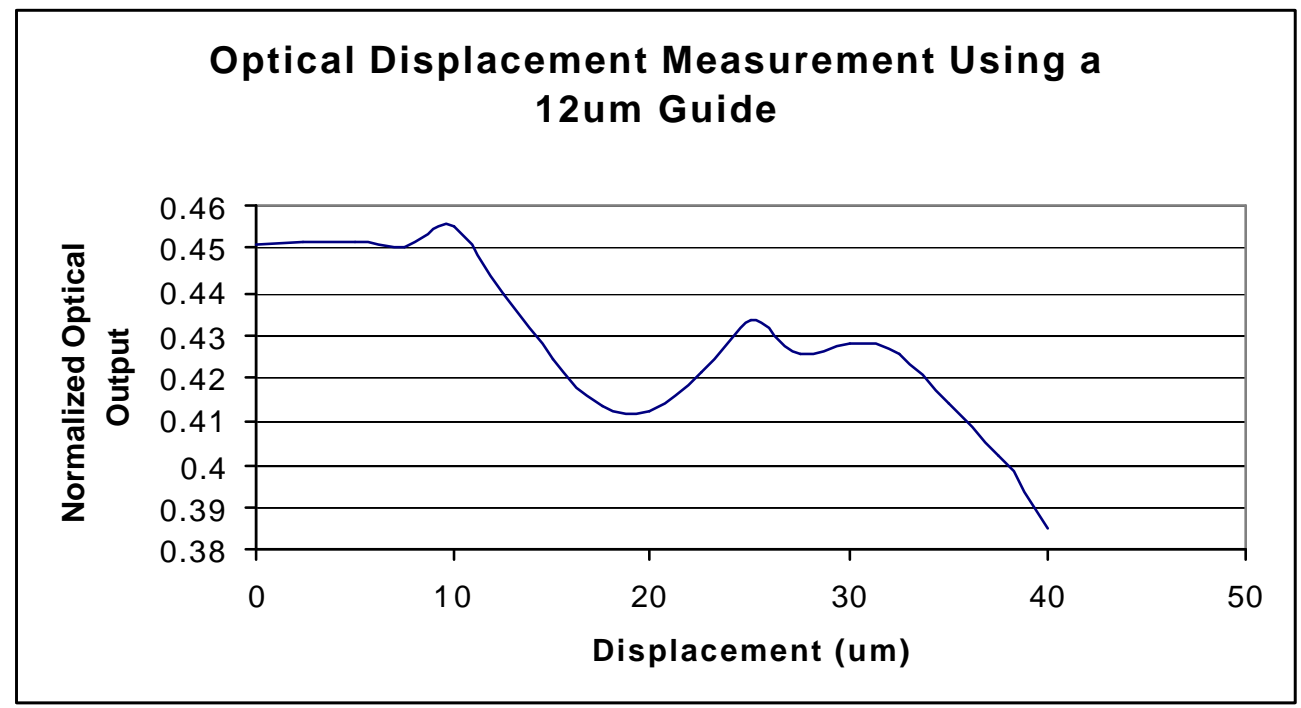

Figure 7-22: Simulated Optical Output using 12 Fm Guide. 


\section{Chapter 8}

\section{Optical Waveguide Co-Integration}

The goal of this research is to explore the feasibility of using optical waveguides for planar IOM of MEMS devices. One aspect of this research is to realize the co-integration process by fabricating optical waveguides with prefabricated linear comb resonators, using the facilities of the micro/nanosystems fabrication facilities at WVU. As presented in Chapters 5 and 7, the use of optical polymers as a waveguide material requires that the waveguide structures be fabricated after the MEMS structures. The ability of the post processing, and the photopatternability of the polymers Exploring the co-integration process required two steps. First was to develop the fabrication process of polymer waveguides at the WVU fabrication facilities, and second was to explore the cointegration of the waveguide probe structure fabrication with the pre-fabricated MEMS devices fabricated at MCNC using the MUMPS foundry service.

\subsection{Ultradel Optical Polymer Waveguides}

Ultradel 9000 series polymers produced by AMOCO were chosen to fabricate the optical waveguides next to the linear resonator structures that were fabricated at MCNC. This polymer choice was primarily dictated by the fact that it was the only commercial polymer available in development 
quantities at the time of this research. The ability to photo-pattern the polymer layers, and the ability to make single mode waveguides, even though the waveguides for this research were predetermined to be multimode, were additional attractive features.

Originally, Ultradel polymers were developed for optical polymer layers and waveguides on a circuit board level, however the fine feature resolution of the material allowed it to be used in chip level applications. The Ultradel polymer was supplied as a developer's kit, which contained a cladding polymer, waveguide core polymer, adhesion promoter, and developer. Ultradel 9020D is a cladding polymer which can be spun-cast into thin films with thicknesses between 2 and $5 \mathrm{Fm}$ after a hard bake. The material acts as a negative photoresist when exposed to UV light $(?=365 \mathrm{~nm}) .9020 \mathrm{D}$ polymer layers have an index of refraction of 1.5479 at a wavelength of $633 \mathrm{~nm}$ and an index of refraction of 1.5310 at a wavelength of $1300 \mathrm{~nm}$. Its complementary waveguide core polymer, Ultradel 9120D, can also be applied in thicknesses of 2 to $5 \mathrm{Fm}$, and acts as a negative resist. The 9120 has a refractive index of 1.562 at a wavelength of $633 \mathrm{~nm}$ and a refractive index of 1.300 at $1.3 \mathrm{Fm}$. Originally the polymer adhesive, which is applied to the substrate before creating waveguide structures, was supplied as two solutions, Ultradel A400/B400, which had to be mixed before application. Later the adhesion promoter was supplied as a single solution, Ultradel A600. The primary component of this adhesion promoter solution is methanol, and the active polymer ingredient was listed as a Trade Secret. The developing solution, Ultradel D902D, is primarily Diglyme, which is a type of ether, and is used for development of both the waveguide core and cladding polymer films [57]. 


\subsection{Waveguide Mask Layout}

Step one in developing a fabrication process for Ultradel polymers required the design and manufacturing of a waveguide mask. This mask contained several banks of waveguides using different waveguide widths and spacings to help in assessing the quality of waveguides that can be fabricated with the Ultradel polymers. The design of the mask was sent to PhotoSciences, Inc. (www.photo$\underline{\text { sciences.com) }}$ to be fabricated into a laser written, chrome on quartz mask.

The waveguide mask was designed to be a 5 inch square which was the largest mask size the aligner could hold. Using Lasi 5.0, a CAD program used to layout mask designs. The waveguide pattern was to cover half of the mask. Then it was mirrored and inverted to create a negative pattern on the other half of the mask The mask layout is divided into four separate banks of waveguides. In the first bank, all the waveguides are $15 \mathrm{Fm}$ wide but the spacing between the guides are different. The first set of guides were 20, $15 \mathrm{Fm}$ set on $20 \mathrm{Fm}$ centers, a second group of 20 guides are set on $25 \mathrm{Fm}$ centers, a third group of 20 guides were set on $30 \mathrm{Fm}$ centers, a fourth group of 20 set on $30 \mathrm{Fm}$ centers, and finally a last group set on 55 Fm centers. These five groups of guides were then repeated to make up the first bank of waveguides.

A second bank of guides was created using $20 \mathrm{Fm}$ guides with various spacings. These also were arranged in 5 groups of twenty guides. The first set of guides were on $25 \mathrm{Fm}$ center spacings, the second set on $30 \mathrm{Fm}$ centers, the third set on $35 \mathrm{Fm}$ center, the fourth set on $60 \mathrm{Fm}$, and the fifth set on $85 \mathrm{Fm}$ centers. These sets of waveguides were also repeated.

A third bank of guides was created using $25 \mathrm{Fm}$ guides, and once again followed the same patterns of 20 in a group. The first set was set on $35 \mathrm{Fm}$ centers, the second set on $40 \mathrm{Fm}$ 
centers, the third set on $45 \mathrm{Fm}$ centers, the fourth set on $75 \mathrm{Fm}$ centers, and finally, the fifth set on 100 Fm centers.

The fourth bank of waveguides were a set of three different waveguide widths. First there are 20 waveguides that are $15 \mathrm{Fm}$ in width set on $80 \mathrm{Fm}$ centers, then there are 20 waveguides that are 20 Fm wide set on $85 \mathrm{Fm}$ centers, and finally there are 20 waveguides that are $25 \mathrm{Fm}$ wide that are set on $100 \mathrm{Fm}$ centers.

\subsection{Ultradel Waveguide Process Flow}

A general guideline for Ultradel waveguide fabrication was provided by AMOCO. This process is outlined in Figure 8-1. Step one is to prepare the substrate by applying a thin layer of adhesion promoter on clean wafers. Following the substrate preparation, the cladding polymer is then applied to the surface using a spin-cast techniques which will produce the desired thickness of film. The cladding polymer is soft baked and then exposed 300 to $900 \mathrm{~mJ} / \mathrm{cm}^{2}$ of UV light of wavelength of $365 \mathrm{~nm}$ to pattern it. Once the material has been exposed for the desired amount of time, the material must be baked before development to improve feature resolution. The film is then spray developed and hardbaked at $300 \mathrm{EC}$ in a furnace for 30 to 60 minutes. A second polymer, the waveguide core, can be applied in a similar manner and processed using the same steps [57].

This general Ultradel process flow needed to be modified to the equipment in the lab at WVU. In some cases, this modification consisted of refining times and temperatures to achieve maximum feature resolution, but in other cases, modifications consisted of creating new 


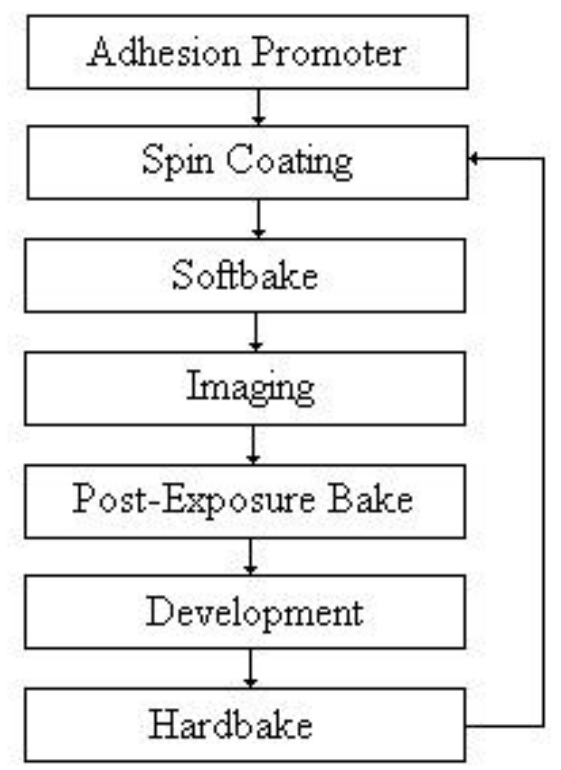

Figure 8-1: Ultradel Process Flow

equipment for the lab. All of this work was carried out in ERB309, the micro/nanosystems fabrication laboratory, . A complete outline of the process flow is shown in Figure 8-2.

The first part of the fabrication process is to clean the wafer or substrate. Two inch, silicon wafers were chosen for this characterization process because they use less chemicals for processing and are easier to handle. To start the cleaning process, the wafers are inserted in a hot SummaClean bath for 30 minutes. SummaClean (produced by Mallinkcroft Transistar) is a choline based solution that removes heavy metal ions from the surface of the wafer. The optimum cleaning temperature is between 50Eand 60EC. After the SummaClean bath, the wafers are inserted in a continuous DI bath for five minutes. Once the wafers are removed from the water, they are blown dry with nitrogen, and inserted in a 100EC furnace for at least 30 minutes for a 
Wator Clesuing

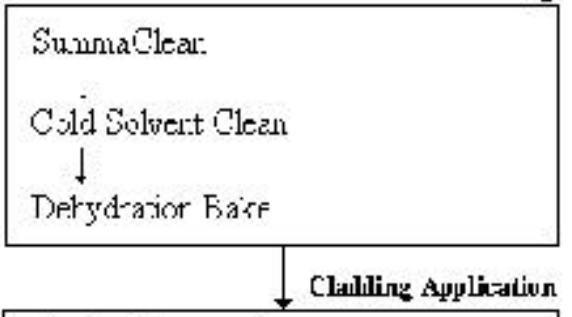
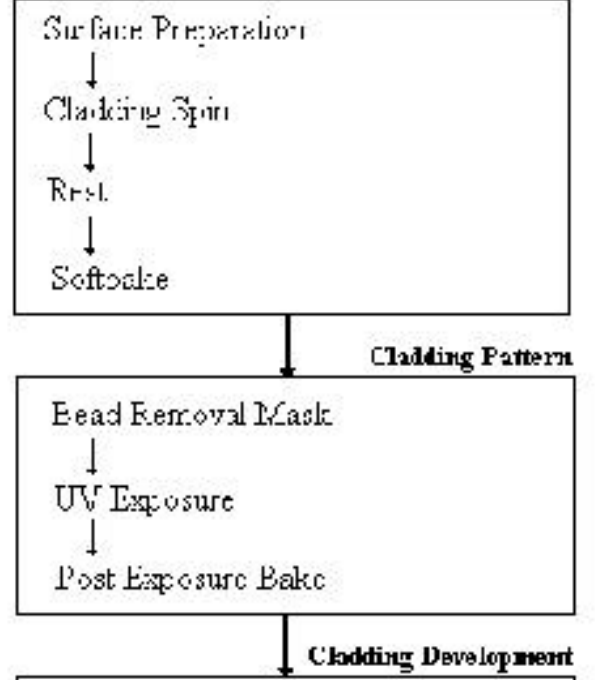

$$
\begin{aligned}
& \text { Dexicpemen: } \\
& \downarrow \\
& \text { H:- }
\end{aligned}
$$

Fateg uilk Syplicaún
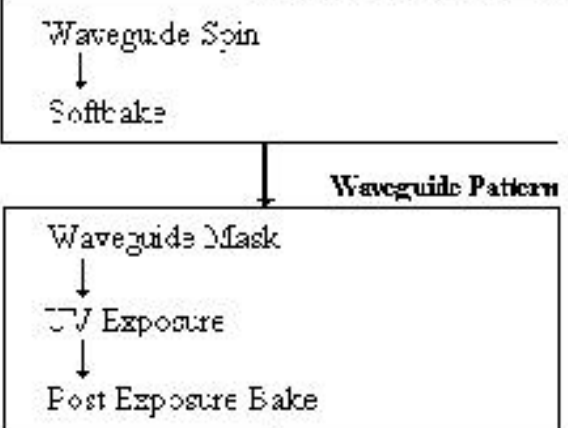

Wavezuide Derclopment

Develspement

$\downarrow$

Harithake.

Figure 8-2: Final Ultradel Polymer Waveguide Fabrication Process Flow 
dehydration bake. At this point the wafer substrates are scribed with two parallel lines along the crystal axis so that the wafer and waveguides could be cleaved after the processing was done.

After a SummaClean, the wafers are given a cold solvent cleaning process. There are two reasons for using this process: first, AMOCO recommended using this clean to improve adhesion of the polymers, and second, this solvent process is part of the resist strip required on the MEMS die. Since the die will be shipped unreleased they were supplied with a protective coating of photoresist which has to be removed before additional processing or release. The solvent clean consists of soaking the wafers for five minutes in Acetone, five minutes in Methanol and five minutes in DI water. When the wafers are moved from one bath to the next, they are kept wet. Once the wafers are removed from the DI bath, they are blown dry with nitrogen and inserted into a 100EC furnace for at least one hour for a dehydration bake.

If the wafers are not going to be used until the following day, they may be left in the furnace overnight. If the wafers needed to be stored for a longer period of time, they should be kept in a nitrogen purged dry box. Before applying a cladding layer to silicon, silicon dioxide, silicon nitride or ceramic substrates, an adhesion promoter must to be applied. To apply the promoter to the wafer, it is centered on the chuck in the photoresist spinner station. Spinner settings were adjusted so that the spin will be preceded by a spreading cycle. The spread is set to spin at 500 RPM for 0.5 seconds and then the spinner will increase spindle speed to 5 KRPM for 30 seconds. Adhesion promoter, Ultradel A600, is applied to the wafer surface with a pipette and then the wafer was spun. Afterwards, the wafer is placed on a 100EC hotplate for one minute to drive off all solvents in the adhesion promoter. 
The wafer is removed from the hotplate and repositioned on the spinner chuck for the cladding polymer application. Ultradel $9020 \mathrm{D}$ can be spun at different rates to produce films between 2 and 5 Fm in thickness. For this application a layer of $2.5 \mathrm{Fm}$ was chosen, so the spinner is set for a spread cycle of 500 RPMs for five seconds and a spin cycle of 4 KRPM for 60 seconds. A syringe is employed to apply the cladding polymer solution to the surface of the wafer. The spinner is then turned on and the polymer film is spun out evenly on the surface of the wafer. Originally at this point, the wafer is placed back on the hotplate for a soft bake, however initial waveguide trials revealed difficulties in adhesion around the edges of the wafer. It was noticed during initial experiments that the polymer films would curl and experience lift-off along edges of the wafers The curling of the films indicated a stress inside them.

To correct this problem, a process delay is performed after the cladding layer is spun onto the wafer. Once the spinner stops, the wafer is allowed to rest for one minute before transferring it to the hotplate. This period of rest serves two purposes that remove the adhesion problems: first, the rest allows the cladding polymer time to bond to the adhesion promoter, and secondly, the rest allowed time for the polymer to conform to the wafer surface and reduce internal stresses in the film. An additional advantage is created by allowing time for the film to rest, in that the relaxation of the films allows the edge bead of polymer, which is created during the spinning, to smooth out some. While not reducing the edge bead entirely, it did reduce to half its original height, and therefore reduced topology of the film, enabling better mask contact. Before exposing the film to pattern it, the wafer is placed on a $100 \mathrm{EC}$ hotplate for three minutes as a softbake. This bake drives off some of the solvents in the film and removes the tackiness of the surface of the polymer. 
The cladding layer does not be need to be patterned since this process development is using a bank of waveguides, however a bead removal mask was created to remove all of the edge bead on the wafer. This would create a smooth even substrate for patterning the waveguide film, reducing diffraction effects common in mass with repetitive patterns. A $1 \frac{7}{8}$ inch circle was cut in black matte board to create the mask. When the wafer is positioned in the aligner, the matte board mask is positioned over the mask holder. The film is exposed to UV light with a $365 \mathrm{~nm}$ wavelength for 50 seconds. Originally the aligner station was operating at a wavelength of $310 \mathrm{~nm}$, but the internal mirrors (which act as a wavelength filter) were changed so that the exposure wavelength would be 365 $\mathrm{nm}$.

Once the polymer film is exposed it is placed in a 170EC, nitrogen purge furnace. This post exposure bake is necessary to retain feature resolution. Its purpose is to harden the surface of the film and make it resistant to the developer, if the cladding polymer is not hardened, it absorbs some of the developer and swelling and distortion is created in the features. The purpose of the nitrogen atmosphere in the furnace is to displace any moisture in the furnace environment which might be absorbed by the polymer. While the cladding layer does not have fine features, it requires development to remove the edge bead.

The exposed and baked polymer must be developed with the Ultradel D902D, which is an ether based developer. Originally, the film development was attempted by dunking the wafer into a bath of developer, however this method was inefficient at removing the unexposed material from the features and there was a significant swelling of the material, which destroyed the surface of the polymer films. To perform spray development in the spinner station an experimental spray development setup was created. 
The exposed wafer is centered on the spinner chuck inside the spinner hood to develop it, and the spinner is set to spin at $1 \mathrm{KRPM}$ without a spread cycle. The timer on the spinner is not set, so the development spin could be timed manually by the operator. After starting the spinner, the spray development is initiated by turning on the nitrogen flow. As the wafer spins, the nozzle is manually moved from side to side to assure that the entire surface of the wafer is being sprayed with developer. This development spray is continued for one minute and five seconds. After the spray is turned off, the spin rate is increased to $4 \mathrm{KRPM}$ an additional minute to allow the wafer to dry off via evaporation of the developer. These timings of the development steps yielded optimum feature resolution for all patterned films.

Once the wafer is dry, it is hard baked in a nitrogen purged furnace set at 300EC. The wafers are left in the furnace for 75 minutes and then removed to cool. At this stage either a waveguide polymer film is applied and patterned, or if the waveguide application has to be delayed, the wafers can be stored in a nitrogen dry box overnight.

The wafer is once again centered on the spinning chuck in the spinner station, and the waveguide core polymer, Ultradel 9120 , is applied to the surface of the wafer using a syringe. It is then spun at a spread speed of 500 RPM for five seconds and a spin speed of 5 KRPM for 60 seconds. This spin rate produces a $1.8 \mathrm{Fm}$ film. Unlike the cladding spin, there is no need for a rest between the spin-cast and softbake, so the wafer is immediately removed from the spinner chuck and placed on a 100EC hotplate for 30 seconds to drive off some of the solvents. Once baked, the polymer film is ready for patterning. 
Both the wafer and the waveguide mask are placed in the UV aligner. Since the polymer acts as a negative resist, the wafer is positioned under the half of the mask which contains the negative waveguide patterns. Minimal alignment is necessary, since care is taken to align the the wafer flat in parallel with the waveguides. The wafer is then placed in contact with the waveguide mask and contact pressure is increased to 2.5 pounds. Exposure time is set for 44 seconds. After the polymer film is exposed, the wafer is removed from the aligner and placed in a $170 \mathrm{EC}$, nitrogen purged furnace for 30 minutes for a post-exposure, pre-development bake.

Once the film has been hardened in the bake, it is ready for a spray development. Again the wafer is centered on the spinner chuck in the spinner station, and the spinner is set for a speed of 1KRPM. When the wafer is spinning, the nitrogen flow is started to start the developer spray nozzle and the surface of the wafer is sprayed for one minute and five seconds. After the spray development is stopped, the speed of the spinner is increased to a speed of 4 KRPM and the wafer is spun for an additional minute to dry the wafer surface. The polymer film is then hardbaked for 60 minutes in a $300 \mathrm{EC}$, nitrogen purged furnace.

\subsection{Fabricated Ultradel Polymer Waveguides}

An SEM photograph of fabricated waveguides created in the micro/nanosystems fabrication laboratory at WVU is shown in Figure 8-3. The waveguides in this photo are $25 \mathrm{Fm}$ wide and set on $100 \mathrm{Fm}$ centers, which are the largest waveguides with the largest spacing between guides that can be fabricated using the mask.

A cross section of one of the $25 \mathrm{Fm}$ waveguides is measured using the AlphaStep, which is a stylus profiler, and is shown in Figure 8-4. The top of the waveguide is not flat, but is shaped with 


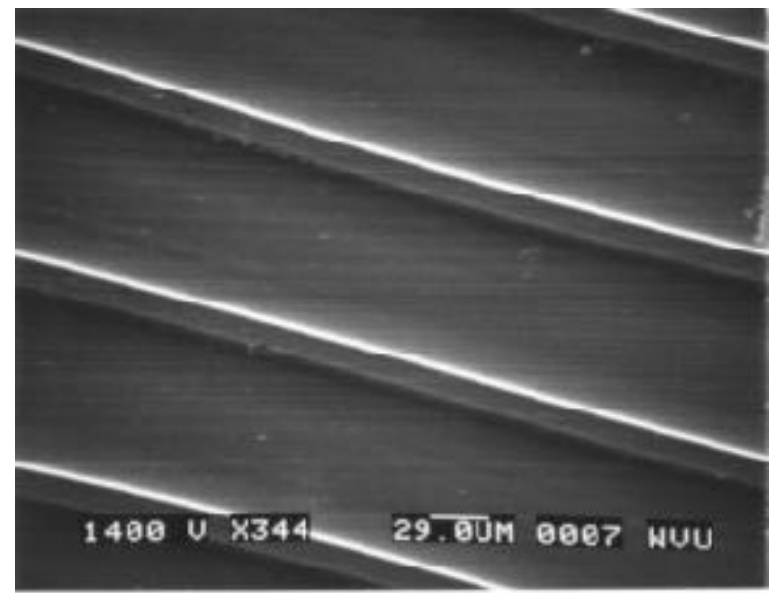

Figure 8-3: 25Fm Ultradel Polymer Waveguides

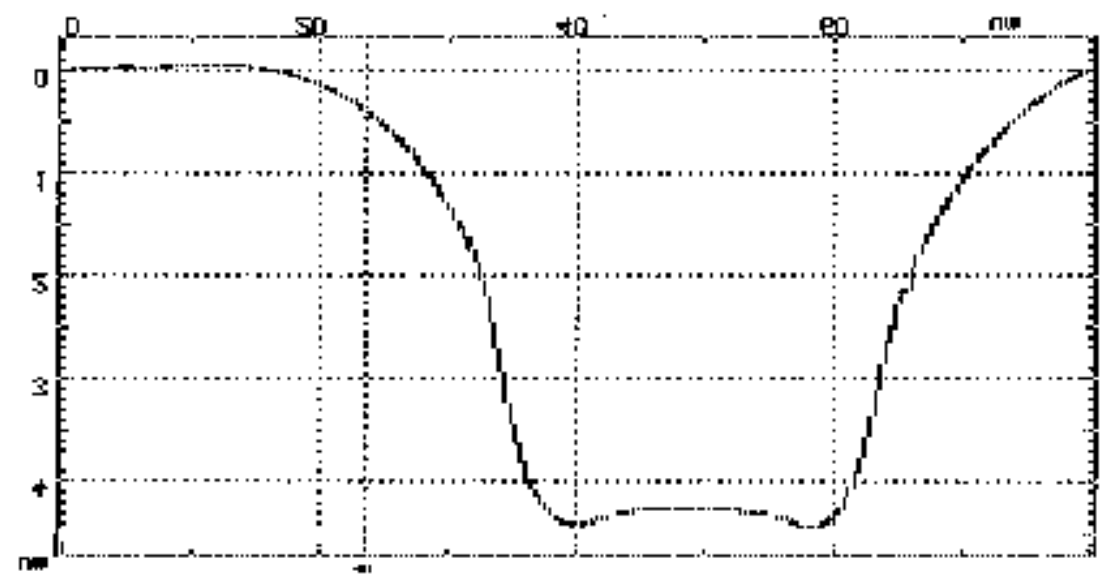

Figure 8-4: AlphaStep Profile of a 25 Fm waveguide.

horn-like structures on the top surface of the waveguides are believed to be created by the swelling that occurs when the polymer absorbs the development solution.

A $25 \mathrm{Fm}$ waveguide was activated by a He-Ne laser with a wavelength of $632.8 \mathrm{~nm}$. Before fabricating the waveguides, the wafer was scribed with two parallel lines, and after the waveguides were fabricated, the wafer was snapped, cleaving the waveguides. The laser was focused into the cleaved 
endface of the waveguide. Visually there was not significant optical loss over the entire length of the waveguide, which is about $25 \mathrm{~mm}$. The abnormal profile of the waveguide did not seem to affect its performance.

\subsection{Prefabricated Linear Resonator}

The feasibility of polymer optical waveguide co-integration with a MEMS linear comb resonator was examined experimentally. Linear comb resonator structures were fabricated at MCNC utilizing their Multi-User MEMS Process (MUMPS). These MEMS devices were a third phase of MEMS design at West Virginia University. A sample layout of one of the linear resonators is shown in Figure 85. This devices is one of 16, that are laid out on a die. Space was left to the right or left of the die for the waveguide probe structures. A scanning electron microscope photo of the fabricated device is shown in Figure 8-6.

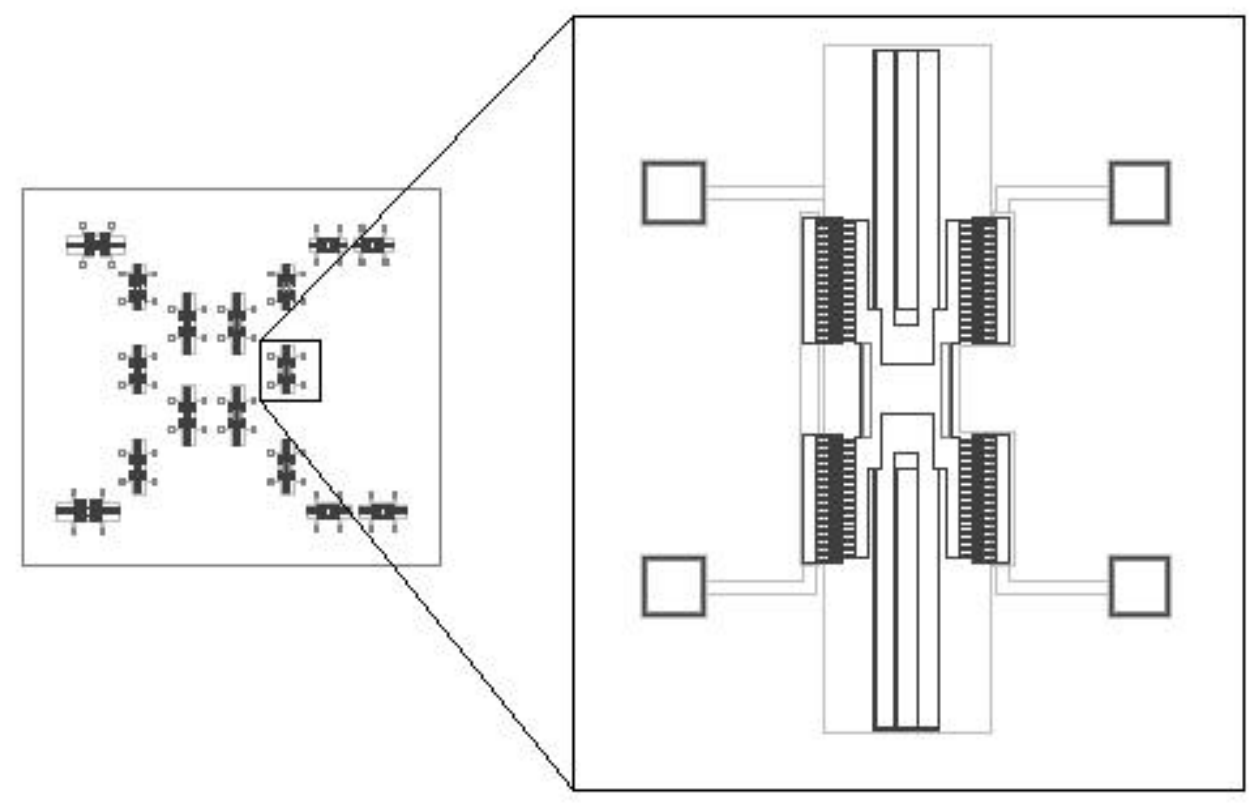

Figure 8-5: MEMS Die with Close-Up of Linear Resonator Structure 


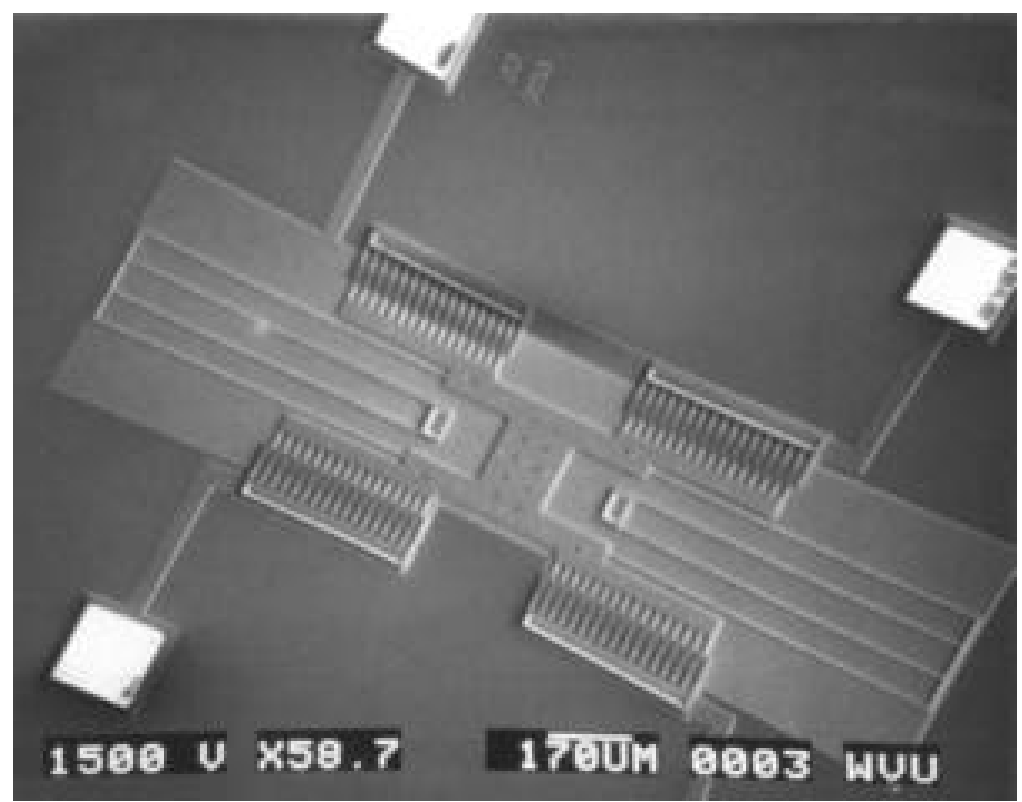

Figure 8-6: SEM Photograph of Fully Released Linear Comb Resonator.

\subsection{MUMPs Process Overview}

A process flow for the fabrication the linear comb resonator device in Figure 8-5 and 8-6 is shown in Figure 8-7. The MUMPs process is called a two-layer, surface micromachining process, because the maximum number of released structural layers is two [12].

Step one of the MCNC MUMPs process is to deposit a $0.7 \mathrm{Fm}$ thick layer of silicon nitride by low pressure chemical deposition (LPCVD) on the surface of the silicon wafer. This film acts as a passivation layer to electrically isolate the MEMS devices from the silicon substrate and to act as an etch stop during the Hydroflouric Acid (HF) release. Next a film of polysilicon is deposited on top of the nitride using LPCVD. This $0.5 \mathrm{Fm}$ polysilicon film is patterned using reactive ion etching (RIE) to form both grounding planes under the MEMS devices, and electrical interconnects lines with the bonding pads. This layer is often referred to 
Silicon Wafer with $0.7 \mu \mathrm{m}$

Nitride Film

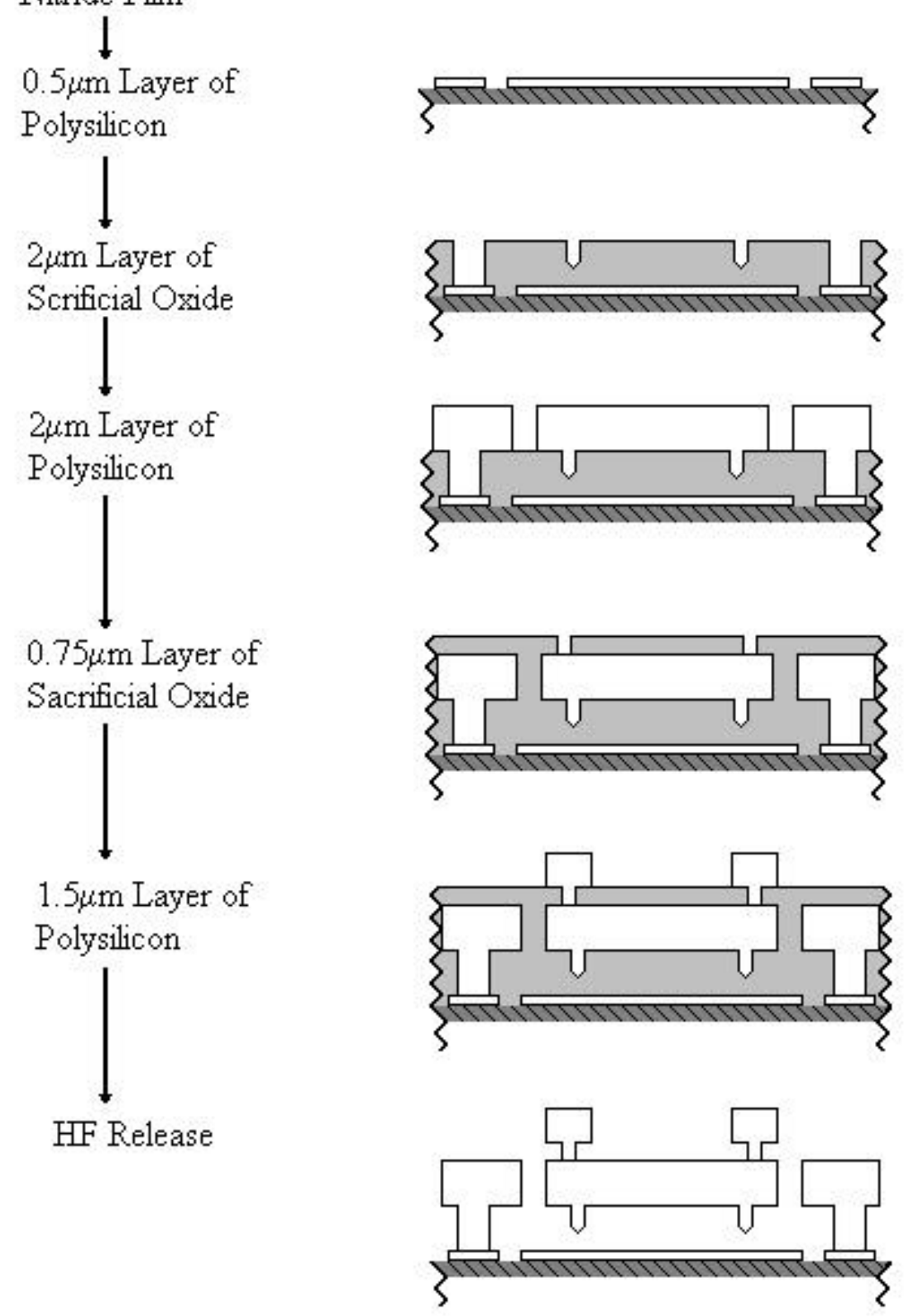

Figure 8-7: MEMS Fabrication Process Flow 
as Poly0. The next film, the Oxide1, is a 2 Fm thick film of silicon oxide that is deposited by LPCVD on the surface of the wafer and patterned using RIE. This oxide layer is a sacrificial layer that will be etched away later; therefore, the patterning of this film creates the negative space that will exist between the structures. Two distinctive features that are formed at this stage are vias which allow that next polysilicon layer to bond with the Poly0 layer, and "dimples" [12].

Dimples refer to small vias that are only $750 \mathrm{~nm}$ deep. Their purpose is to create small stilts under large MEMS structures, and reduce stiction by maintaining a small gap between the MEMS structures, the resonator stage in our structure, and the substrate during the HF release and DI rinse step at the conclusion of the die processing. On top of the Oxide1 layer, a $2 \mathrm{Fm}$ layer of polysilicon, known as Poly1, is deposited. This structural layer is patterned into the linear resonator device: the stage, comb fingers, flexures, and comb drives. A few small etch holes are patterned in the stage to assist with the $\mathrm{HF}$ release. The next layer is the Oxide2 layer, which is a $0.75 \mathrm{Fm}$ thick film of sacrificial oxide. Normally this layer is patterned for contact to a third layer of polysilicion; however, this is not necessary in our devices [12].

On only five of the linear resonator devices on the die, two channels are opened up on each side of the linear resonator stage to create some super structures. The purpose of the structures was to add extra topology to the devices and to extend the vertical height for optical reflection. Vias over the bonding pads are patterned into the Oxide2 layer. The final structural layer, Poly2, a $1.5 \mathrm{Fm}$ layer of polysilicon is deposited onto the surface of the wafer. This layer is patterned to form the superstructures on the linear resonators, and on the bonding pads. After all the structures are formed a gold/ chrome metalization is performed to create contacts on the bonding pads [12]. 
After the MEMs structures are completely fabricated they can be released by soaking the devices in $50 \% \mathrm{HF}$ for 2.5 minutes. Once they are removed from the HF, they should be rinsed in a continuous DI bath for a minimum of 10 minutes. Because the water will cause stiction, the devices should be soaked in isopropyl alcohol for 5 minutes after the DI bath. The die is then removed from the alcohol and placed in a 100EC furnace for an hour to drive off the alcohol and any remaining water [12].

\subsection{Optical Waveguide Co-integration}

\subsubsection{Optical Waveguide Co-integration Masks}

The co-integration of the optical waveguide probe structures with the prefabricated linear resonators required three mask layouts: an oxide removal mask, a cladding mask, and a waveguide mask. These two mask layouts are show in Figure 8-8. All of the masks are dark field because the polymers act as negative resists, which means that only the exposed areas of polymer are left after development. The alignment of the two polymer layers, waveguide core and cladding, with the linear comb resonators is shown in Figure 8-9. Before fabricating the waveguide on a linear comb resonator die, these masks were used to fabricate the waveguide IOM structures over silicon.
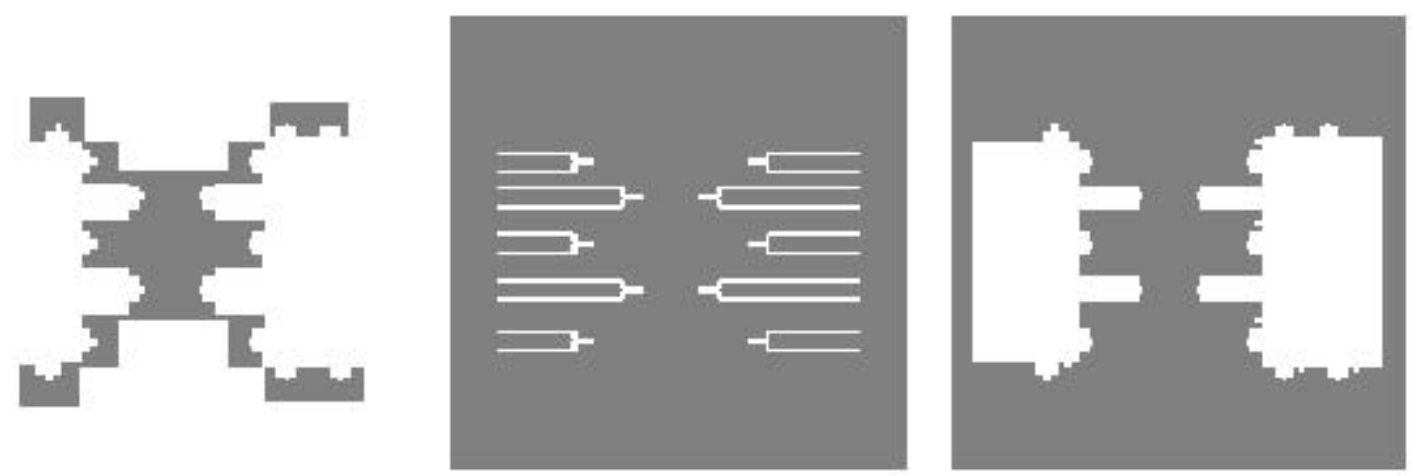

Figure 8-8: Oxide Removal Mask, Waveguide Mask and Cladding Mask. 


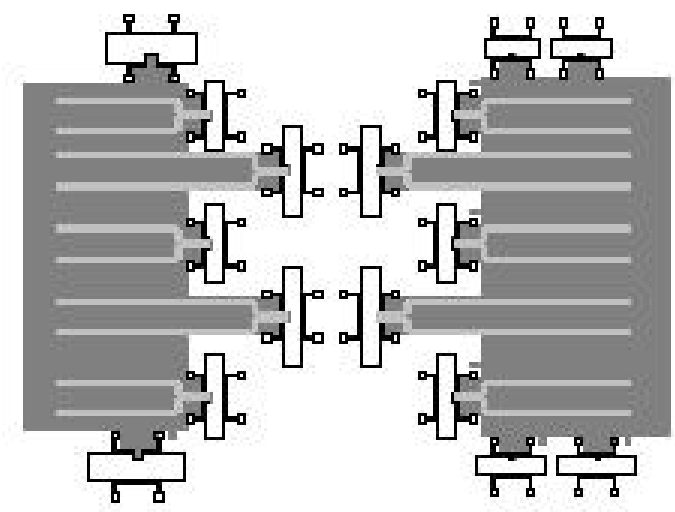

Figure 8-9: Co-Integrations of Linear Resonator, Cladding and Waveguide core.

\subsubsection{Ultradel Waveguide Co-Integration}

The die were received from MCNC unreleased. Covering the polysilicon structures was a thick coat of silicon oxide and a thick layer of photoresist. The thick photoresist was applied to protect the die during dicing and shipping. Before any waveguide fabrication is performed on the die, the photoresist must be removed. Following the recommendations of MCNC, the die are soaked for 20 minutes in acetone. To assist the removal process, the beaker of acetone is placed in an ultrasonic bath. When the die is removed from the acetone, it is soaked in methanol for five minutes. After the methanol soak, the die is placed in a continuous DI bath for an additional five minutes. The die are baked in a $100 \mathrm{EC}$, nitrogen purge furnace for thirty minutes for a dehydration bake. A process flow for the waveguide co-integration process is shown in Figure 8-10. 
Before processing can be performed on the die surface, it must first be mounted on a substrate wafer so that it can be easily handled by the spinner and aligner. Each die is a $10 \mathrm{~mm}$ square piece of silicon, and this size is too small to properly fit on the machine's vacuum chucks. Since there were many monitor grade two inch wafers available, and the lab equipment is rated to handle that size of wafers, a two inch wafer was used as a mounting substrate. The die is mounted on the wafer using photoresist. A drop of photoresist is applied in the center of the wafer using a pipette and then the die and shards were positioned on top of the resist. To avoid creating a bead on the edge of the die, which is more pronounced on non circular substrates, silicon wafer shards are positioned to butt up against the edges of the die. This is illustrated in Figure 8-11. To harden the resist, the wafer is then placed on a 100EC hotplate for one minute and then baked in a 100EC furnace for thirty minutes.

Once the photoresist is stripped off the die and properly mounted on a silicon wafer, the next step is to prepare it for an oxide etch. The sacrificial oxide layer, which is a phosphorosilica glass (PSG), needs to be removed from portions of the die where the polymer waveguides will be fabricated. Consideration was given to using the oxide as a cladding layer for a waveguide, however there were some difficulties with this idea. The waveguide would not be a sufficiently wide enough mask to stop the HF from under etching the oxide during a release. Secondly, the object of the waveguide fabrication was to place the waveguide in the same plane as the resonator stage, which meant the oxide needed to be removed in part. Therefore a photoresist mask is applied and patterned using the oxideetch mask before the etch. 


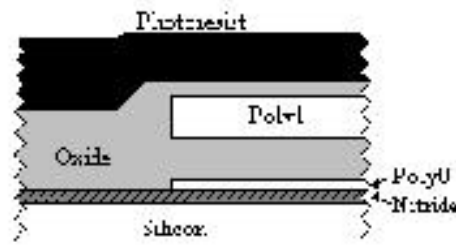

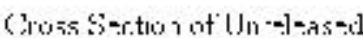

Linzar Kc3onator Struztice

with a trisl: 20 at $\circ f$

Protoresst.

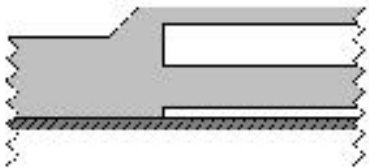

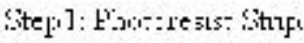

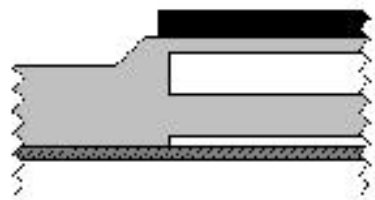

S.er 2. Fres: P:Jouresl

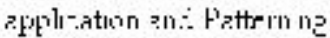
to form ct:h _Mask.

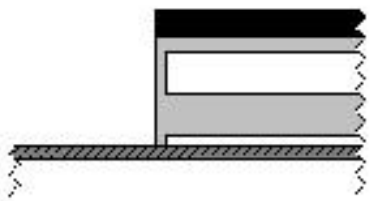

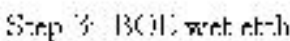

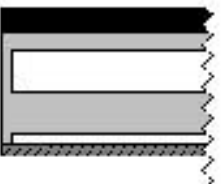

Stes 4 RE rixide etch

$\vdots$

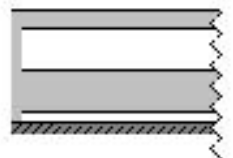

S-eF 5: Ptotoresiat

terruvid.

$\vdots$

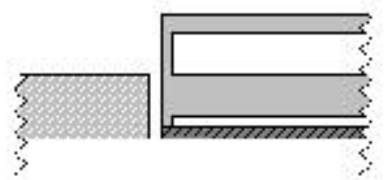

Step \&: Caciting Porner asplicat:or. and patterninc.

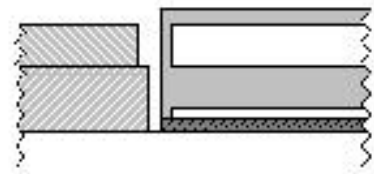

Stco? War:gxisc U'crc Folyre: applcation ard sateriag.

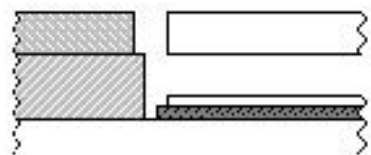

Step 8: HF release

Figure 8-10: Waveguide Co-Integration Process Flow. 


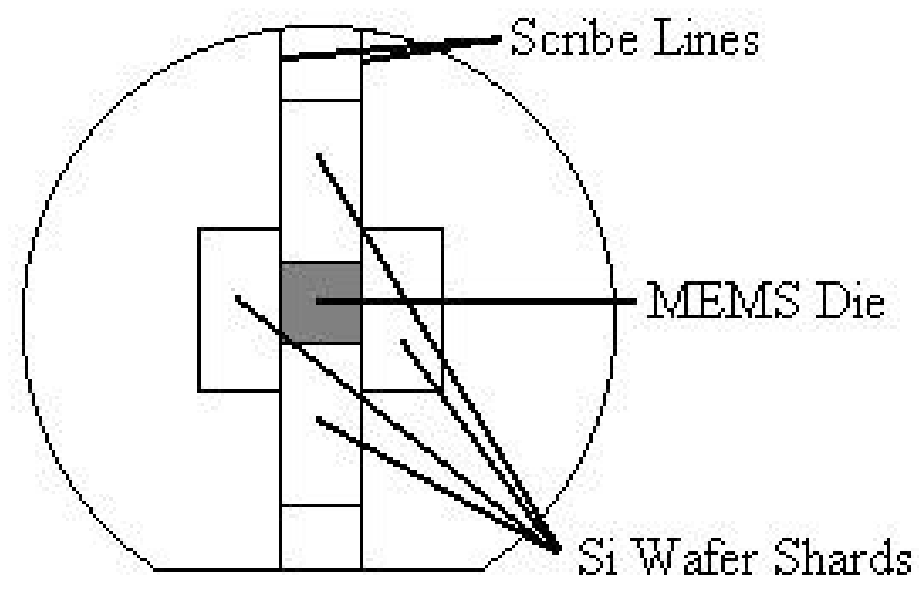

Figure 8-11: MEMS Die Mounted on Substrate Wafer.

Since the top surface of the die is a silicon oxide, the die needs to be primed with an adhesion promoter. The wafer and die are placed into the HMDS furnace which coats the surface with a a thin film of Hexamethyldisalisane. When the die was cool, it and the wafer are place on the spinner chuck to apply a layer of photoresist. The spinner settings were set to produce a spread cycle off 100 RPM for 5 seconds and a spin of 4.5 KRPM for 30 seconds. This spin speed will produce a $1.2 \mathrm{Fm}$ thick film of photoresist. The photoresist is applied to the surface with a pipette and then the spinner is started. After the spin, the wafer is removed from the chuck and placed on a 100EC hotplate for one minute to soft bake the resist.

The photoresist is to be used as an etch mask for a wet etch of the underlying silicon dioxide. $\mathrm{SiO}_{2}$ in the areas under the cladding must be removed, so the photoresist is patterned using the cladding layer mask. The mask is open in the areas the cladding will remain, since the polymer acts as a negative 
resist. This open area is the oxide that will be etched away. The mask is placed in the aligner with the MEMS die wafer. Alignment of the MEMS structures to the mask was critical, and difficult, since the mask hid the underlying features. Once alignment is achieved, the die is placed in contact with the mask using a pressure of 2.75 pounds. Exposure time is 3.5 seconds. The wafer and die is then removed from the aligner and the photoresist is developed, using a 3:1 solution of DI water to development solution. After development and inspection, the photoresist is hardbaked in a 100EC furnace for 30 minutes.

Now that the photoresist mask has been hardened the oxide can be etched. The wafer is submerged in a Buffered Oxide Etchant (BOE) bath for 30 minutes with only occasional agitation in order to inhibit underetching of the PSG. When the wafer is removed from the bath, it is rinsed in a continuous DI bath for a minimum of 10 minutes. The wafer is then removed from the water and dried with a nitrogen gun. The $\mathrm{BOE}$ weakens the photoresist mask but does not remove it entirely. At this time, the die is lifted off the wafer by using a razor blade to pry off the shards and die.

Originally, application of the cladding film to the silicon nitride was planned. One purpose of the nitride is to act as an etch stop for the HF during the release process. When cladding and waveguide films were fabricated on the nitride and then subjected to a release process, the polymer films lifted off in the DI rinse bath. When the die was inspected more closely, the surface of the die substrate appeared to have undergone a change. Besides the visual surface difference, measurements taken using the Nanospec, which measures film thicknesses by interference, had shown the nitride thickness had been reduced. It is believed that the HF had partially etched away the nitride as a result of the 2.5 minute exposure to straight $\mathrm{HF}$. The inability of the nitride to resist the HF acid and the previous success 
achieving adhesion on bare silicon meant that removal of the nitride from the die in the areas that the cladding would be applied may solve this problem.

Therefore, after the oxide on the die was etched away, the nitride was fully etched in a Flouroform (Halocarbon-23) and Oxygen plasma in the Oxford Instruments reactive ion etcher. The plasma was maintained at a pressure of 55 mTorr and a power of 140 Watts. A Halocarbon-23 rich plasma was desired, so the gas flows were set for $75 \mathrm{sccm}$ of Halocarbon-23 and $5 \mathrm{sccm}$ of Oxygen. Ideally this plasma etch should be performed using a Silicon Graphite cover plate for the RIE head [60]. The Quartz cover plate was substituted for the Graphite plate since one was not available. Difficulties created in the etching by the change in cover plate materials will be discussed later in this chapter. The die was placed in the RIE and a process run was performed. The best time length for the nitride etch was found to be 27 minutes via experimentation.

After the nitride was removed it was time to apply the optical polymers to construct waveguides. In general the fabrication process for the polymer waveguides followed the outline for the Ultradel process described in Section 8.3. A few modifications had to be made to the process. First, the die is cleaned utilizing the cold solvent clean of 5 minutes acetone, 5 minutes methanol, 5 minutes DI, and a 30 minute dehydration bake in a 100EC. Afterwards it is remounted on a two inch wafer substrate, which is pre-scribed with two parallel lines that are $10 \mathrm{~mm}$ apart. The die is mounted on this substrate between the scribe marks using some 9020 polymer as an adhesive. To get an even coat of polymer, for mounting, a small amount of 9020 is applied and spun cast on the wafer. It is then removed from the chuck and the die is mounted between the scribe marks. The scribe marks will allow the wafer to be cleaved away later. Four shards are positioned around the wafer, similar to the earlier wafer mounting. 
Once all pieces are mounted on the surface, the wafer is placed on a 100EC hotplate for 1 minute to solidify the film. Then the wafer is baked in a $300 \mathrm{EC}$ furnace for 30 minutes to anneal the polymer.

Once the wafer was cooled, it is remounted in the spinner chuck for an adhesion promoter spin and a cladding layer spin. The fabrication steps followed are those listed earlier. The desired thickness of the cladding is $3.1 \mathrm{Fm}$, which is the thickness of the removed silicon nitride and Oxide1 layer, so the spinning speed is set for 3.4 KRPM [12]. This thickness of the polymer will vertically align the waveguide core with the linear resonator stage edge. Patterning the cladding is different than earlier, because the cladding pattern requires a mask. Now the die has to be aligned to the mask. Once alignment is achieved, a contact pressure of 2.5 pounds is applied and the UV exposure time is 44 seconds. The cladding film is then post-exposure baked, developed and annealed.

The next layer to apply is the waveguide core polymer. The process is repeated the same as described above. Waveguide thicknesses needs to be $2 \mathrm{Fm}$, which is the thickness of the linear comb resonator stage, so no changes are made in the application spin speed. The film is spun on, exposed, post-exposure baked, developed, and annealed.

After the waveguides are completely fabricated, the wafer is cleaved along the scribe marks, and then the rest of the wafer is cleaved away. The last step of the process is to release the structures. This procedure is the one recommended by MCNC. The die are soaked in a straight HF bath for 2.5 minutes with gentle agitation. When the die is removed from the HF, it is placed in a continuous DI bath for 10 minutes. After the DI bath the die is soaked in isopropyl alcohol for five minutes to displace the water. Finally the die is baked in a $100 \mathrm{EC}$ furnace for 30 minutes. 


\subsubsection{Results}

As shown in Figure 8-12, the optical waveguide structures were successfully fabricated on a prefabricated MEMS die. This figure shows a polymer waveguide fabricated next to the phase two generation of linear comb resonator designed at WVU. The MEMS device in the pictures was not released. Fabricated polymer structures consistently lifted off the die surface during the HF release, DI rinse, and alcohol soak As discussed in the preceding section, it was believed that the silicon nitride layer was being etched away by exposure to HF.

An experimental path was taken to reactively ion etch the silicon nitride layer to solve the lift-off problems occurring during the release process. During the nitride etch experiments, one set of waveguides were fabricated and successfully released without lift-off. Unfortunately, at that time no

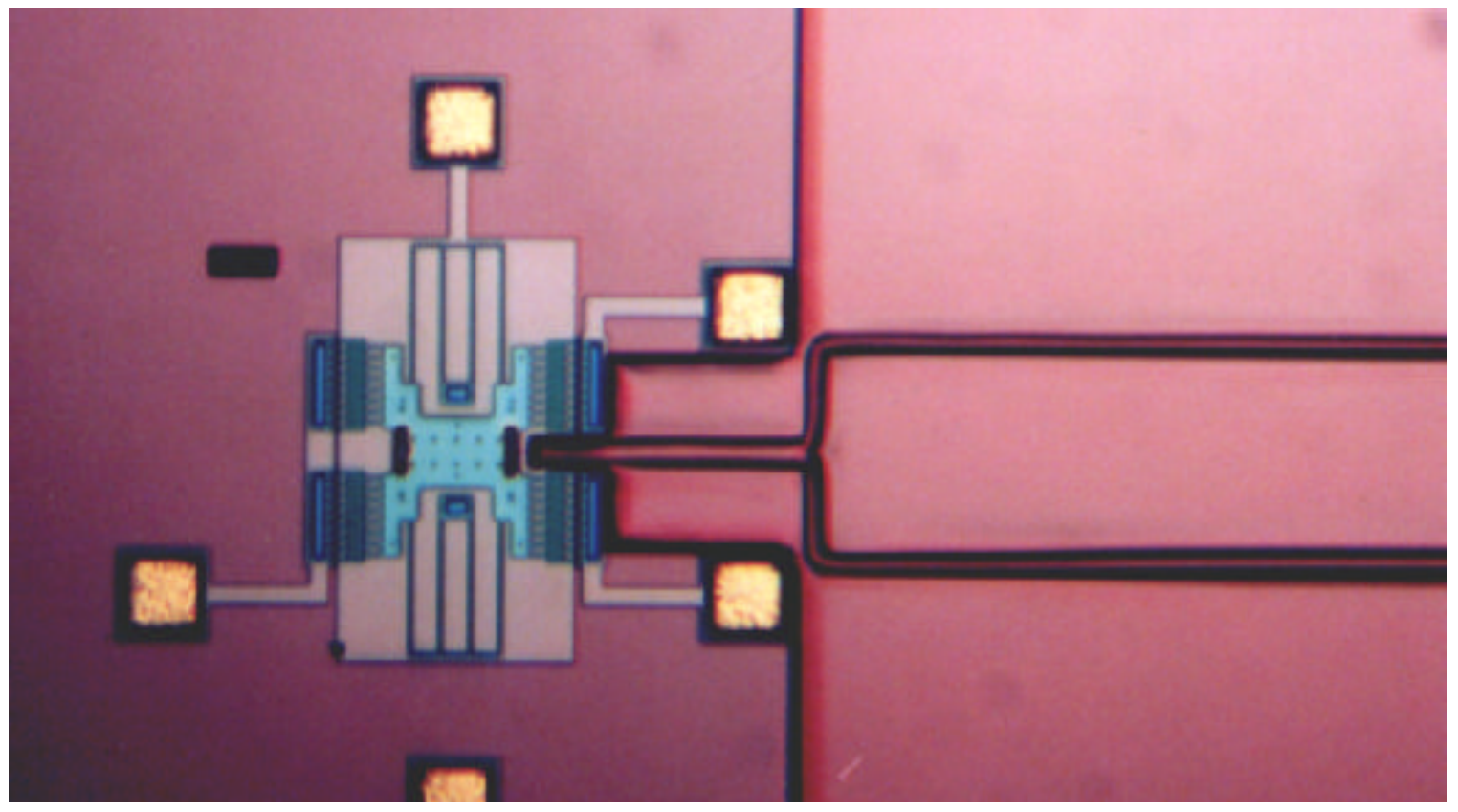

Figure 8-12: Polymer Waveguide and Cladding Fabricate Next to Phase 2 Linear Comb Resonator. 
photo-documentation was taken. However this success encouraged exploration of the RIE nitride removal. The objective of the etch was to remove all the nitride film and expose the bare silicon on the die. Subsequent experimental etches revealed this path to be more difficult than perceived.

The length of the etch time became critical. Too short of etch time would underetch the nitride and the film could possibly leave a barrier of silicon nitride which would still etch away during the HF release process and the polymer waveguide structures would lift-off. Lengthening the etch time to ensure the silicon nitride film was etched away completely would cause over etching. The resist mask that was used to mask the oxide during a wet etch was weakened by the oxide etching. Freon/Oxygen plasma, would eventually etch away the resist and start attacking the sacrificial oxide. If the etch lasted long enough it would eventually attack the polysilicon, MEMS structures. A second problem encountered with overetching was a problem with redeposition. The edges of the die started changing color and showing possible signs of redeposition. The redeposited material is believed to be a silicon oxide or quartz film created by the etching and redeposition of the quartz cover plate. Reactively ion etching the nitride has very tight tolerances and will require a thorough study of the etch process.

\subsection{Future Work}

As of June 1999 Ultradel polymer was no longer commercially available AMOCO had decided to discontinue their manufacturing of the material. The batches of Ultradel Polymers that had been purchased before the discontinuation have passed there shelf life and usability. Figure 8-13 shows a cladding polymer film that was applied with expired solution. To continue the research co-integrating 
optical polymer waveguide structures a new viable polymer must be found and substituted in the process that has already been defined.

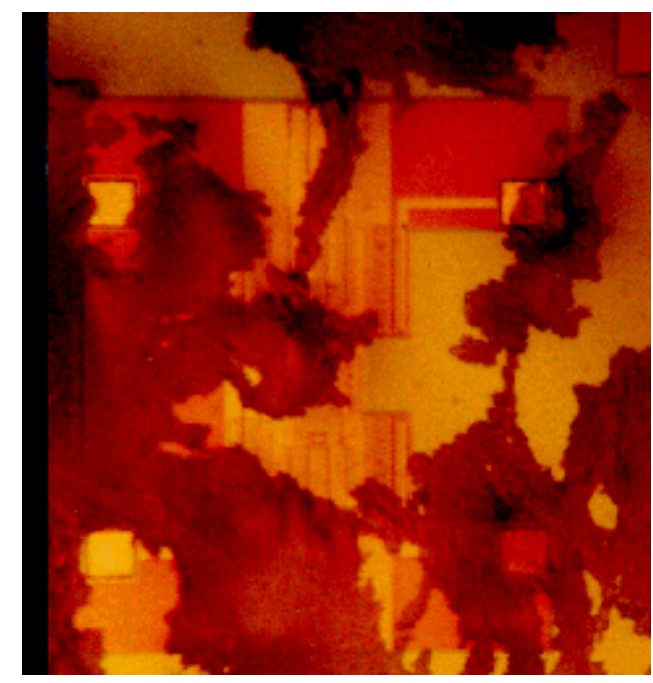

Figure 8-13: Unusable Cladding Polymer Film 


\section{Chapter 9}

\section{Summary and Conclusions}

The goal of this research was to explore the feasibility of using integrated optical waveguides for integrated optical metrology of microelectromechanical structure motion. Work presented here is an initial study which explored the basic issues that face integrated optical metrology and the methods for co-integrating measurement systems. Measurements, simulations and analysis focused on characterizing a comb driven, linear resonator MEMS device. This device was chosen because it is a standard device used in many MEMS systems and it displays linear motion along a single axis. The ability of optical metrology was evaluated by the measurement of displacement, operating frequency, phase shifts and resonant frequency.

Initial, bulk optical metrology studies indicated the effectiveness of guided optics to detect the displacement of the linear comb resonator. Using multimode fibers, the measured signals confirmed device behavior matches the theoretically derived values. A measurement signal representing the motion of the device could be dectected using single mode input dector fiber, and that the device was behaving as expected. This use of a single mode fiber for detection also demonstrated the sensitivity of alignment to external vibrations and the potential of coherent IOM. 
After establishing optical metrology with optical fibers, the research explored the viability of developing an integrated version of the optical fiber measurements. Integrating the bulk optical metrology, will allow in-situ measurements of the device during operation, without interfering with the operation. Developing an integrated optical waveguide structure with the MEMS device using planar waveguides creates two methods of measurement. These two measurement approaches have the potential to yield significant positional information.

Single mode waveguides give the ability to make interference measurements because the emission of a single mode guide is one uniform wavefront, which can interfere with reflections from the linear resonator stage. Both the optical performance simulations and proposed fabrication process for co-integration, are presented. The fabrication of single mode waveguide probe structures can be achieved through MEMS process interuption utilizing iorganic optical materials. Further exploration of the single mode integrated waveguide structure should focus on the optimization of the waveguide layout for minimum power loss and minimum footprint.

Multimode waveguides carry many more modes, and can only be used to measure changes in the output intensity due to optical path length changes created by the motion of the device. The multimode microprobe structures can be fabricated using optical polymers, and a large section of the dissertation discusses the design and fabrication issues of polymer waveguide structures for cointegration. While the development of the polymer waveguide co-integration can create a new basis for optical device co-integration with MEMS structures and expansion of this process can create new micro-optoelectromechanical structures, experimental results indicate that guided wave IOM would best be explored by examining through process interruption of hybrid techniques, given existing polymer 
materials. Additional exploration of multimode guides can include a detailed analysis of displacement measurement with respect to partial mode filling in the guide structure to determine the best dimensions of the waveguide layout. 


\section{Bibliography}

[1] C. A. Ball, M. D. Walters and R. L. Wood, "Contamination issues for MEMS fabrication",

Cleanrooms, pp. 26-31. March 1996.

[2] G. K. Fedder, "Future trends in microelectromechanical systems (MEMS)", Web Page, 1998, http://www.ece.cmu.edu/pubs/vision/mems.html

[3] P. Coy, J. Carey, and N. Gross, "Mighty mites hit it big", Business Week, pp. 92-94, April 26, 1993.

[4] W. B. Scott, "Micro-machines hold promise for aerospace", Aviation Week \& Space Technology, pp. 36-39, March 1, 1993.

[5] R. T. Howe, R. S. Muller,. K. J. Gabriel, and S. N. Trimmer, "Silicon micromechanics: sensor and actuators on a chip", IEEE Spectrum, pp. 29-35, July 1990.

[6] G. Stix , "Micron machinations", Scientific American, pp. 106-116, November 1992.

[7] J. J. Sniegowski and E. J. Garcia, "Microfabricated actuators and their application to optics", in Proceeding SPIE, vol. 2383, pp. 46-64, 1995.

[8] DARPA, "Microelectromechanical systems: a DoD dual use technology industrial assessment", Final Report, December 1995. 
[9] T. A. Core, W. K. Tsang and S. J. Sherman, "Fabrication technology for an integrated surfacemicromachined sensor”, Advanced Device Packaging, pp. 39-46, October 1993.

[10] J. Rhea, “ARPA MEMS program to rely on semiconductor industry”, Military \&Space Electronics, pp. 3, April 1996.

[11] K. J. Gabriel, “Engineering microscopic machines”, Scientific American, pp. 118-121, September 1995.

[12] D. A. Koester, R. Mahadevan, A. Shishkoff and K. W. Markus, "MUMPs design handbook (rev. 4)", Web Page, 1996, http://mems.menc.org/smumps/SMTPOC.html.

[13] “Rotary side drive motor”, Web Page, 1999, http://mems.mcnc.org/figs/sem5.gif.

[14] K. E. Peterson, "Silicon as a mechanical material”, in Proceedings SPIE, vol. 70, pp. 420-437, 1982.

[15] A. Kolpekwar and R. D. Blanton, "Development of a MEMS testing methodology", in IEEE International Test Conference, pp. 923-931, 1997.

[16] J. M. Younse, “Mirrors on a chip”, IEEE Spectrum, pp. 27-31, November 1993.

[17] J. T. Stewart, "Finite element modeling of microelectromechanical structures for sensing applications", in Proceedings SPIE, vol. 2642, pp. 194-205, 1995.

[18] W. Ye, S. Mukherjee and N. C. MacDonald, “Optimal shape design of an electrostatic comb drive in microelectrmechanical systems", Journal of Microelectromechanical Systems, vol. 7, No. 1, pp. 16-26, March 1998.

[19] W. A. Johnson and L. K. Warne, "Electrophysics of micromechanical comb actuators", Journal of Microelectromechancial Systems, vol. 4, No. 1, pp. 49-59, March 1995. 
[20] S. A. Miller, Y. Xu, and N. C. MacDonald, "Micomechanical cantilevers and scanning probe microscopes", in Proceedings SPIE, vol. 1640, pp. 45-52, 1995.

[21] Y. Kim and D. P. Neikirk, "Micromachined fabry-perot cavity pressure transducer with optical fiber interconnects", in Proceedings SPIE, vol. 2462, pp. 242-249, 1995.

[22] R. Maboudian, "Adhesion and friction issues associated with reliable operation of MEMS", MRS Bulletin, pp. 47-51, June 1998.

[23] R. Maboudian and R. T. Howe, "Critical review: adhesion in surface micromechanical structures", in Journal of Vacuum Science Technology B, vol. 15, No. 1. American Vacuum Society, pp. 1-20, January-February 1997.

[24] P. R. Nelson, P. B. Chu, and K. S. J. Pister, "Optical methods for characterization of MEMS device motion", in Proceeding SPIE, vol. 2640, pp. 53-57, 1995.

[25] A. T. T. D. Tran, J. J. Lee, K. Zhang, and Y. J. Lo, "Ultrafine motion detection of micromechanical structures using optical moire patterns", IEEE Photonics Technology Letters, vol. 8, No. 8, pp. 10581060, August 1996.

[26] M. T. Ching, R. A. Brennen and R. M. White, "Microfabricated optical chopper”, Optical Engineering, vol. 33, No. 11, pp. 3634-3642, November 1994.

[27] G. K. Fedder and R. T. Howe, "Multimode digital control of a suspended polysilicon Microstructure", Journal of Microelectromechanical Systems, vol. 5, No. 4, pp. 283-297, December 1996. 
[28] W. D. Cowan, V. M. Bright and G. C. Dalton, "Measuring frequency response of surfacemicromachined resonators", in Proceedings SPIE, vol. 3225, pp. 32-43, 1997.

[29] J. S. Burdess, A. J. Harris, D. Wood, R. J. Pitcher and D. Glennie, “A system for the dynamic characterization of microstructure", Journal of Microelectromechanical Systems, vol. 6, no. 4, pp. 322-328, December 1997.

[30] R. M. Dickey, S. C. Holswade, L. A. Hornak, and K. S. Brown, “Optical methods for micromachined monitoring and feedback", Sensors and Actuators A: Physical, (Printing Date Pending).

[31] M. E. Motamedi, "Micro-opto-electro-mechanical systems", Optical Engineering, vol. 22, No. 11, pp. 3505-3517, November 1994.

[32] A. Tran, G. L. Christenson, A. Lopez, and Y. H. Lo, "Micromachined micro-optic devices", LEOS Newsletter, pp. 10-11, April 1996.

[33] J. M. Dawson, J. Chen, K. S. Brown, P. Famouri, and L. A. Hornak, “Through-wafer interrogation of microstructure motion for MEMS feedback control", in Proceedings SPIE, Vol. 3878, pp. 281-292.

[34] W. C. Tang, M. G. Lim and R. T. Howe, "Electrostatic comb drive levitation and control method", Journal of Microelectromechanical Systems, vol. 1, No. 4, pp. 170-178, December 1992.

[35] D. K. Cheng, Field and Wave Electromagnetics, Addison-Wesley, Reading, Massachusetts, second edition, 1989.

[36] F. W. Sears, M. W. Zemansky, and H. D. Young, University Physics, Addison-Wesley, Reading, Massachusetts, fifth edition, 1978. 
[37] R. T. Wiedner and M. E. Browne, Physics, Allyn and Bacon, Boston, revised edition, 1989.

[38] H. J. Lee, M. Tachiki, C. Lieu, and K. S. J. Pister, "UCLA automated MEMS desing and analysis tools- the design of resonant microstructures”, Final report, UCLA MEMS Transactions, 1994.

[39] Y. Cho, A.P. Pisano, and R. T. Howe, "Viscous damping model for laterally oscillating microstructures", Journal of Microelectromechanical Systems, Vol. 3, No. 2, pp. 81-86, June 1994. [40] V. Barger and M. Olsson, Classical Mechanics: A Modern Perspective, Mcgraw-Hill, New York, 1973.

[41] T. Tamir, Integrated Optics, vol. Seven of Topics in Applied Physics, Springer-Verlag, New York, second edition, 1985.

[42] D. L. Lee, Electromagnetic Principles of Integrated Optics, John Wiley and Sons, New York, 1986.

[43] Y. S. Liu, et. al., "Polymer optical interconnect tehcnology (POINT)- optoelectronic packaging and interconnects for board and backplane applications", Critical Reviews, Vol. CR62, pp. 406-414. [44] D. A. Christensen, "Plasma-etched polymer waveguides for intrachip optical interconnects", in $O$ E/Fibers '87. SPIE-The International Society for Optical Engineers, 1987.

[45] T. Tamir, and S. T. Peng, "Analysis and design of grating couplers", Applied Physics, Vol. 14, pp. 235-254, 1977.

[46] M. C. Tucker, "Polyimides have the right chemistry for MCMs", Hybrid Circuit Technology, pp. 23-28, October 1991.

[47] L. A. Hornak and T. W. Weidman, "Propagation loss of index imaged poly(cyclohexylsilyne) thin film optical waveguides", Applied Physics Letters, Vol. 62, No. 9, pp. 913-915, March 1993. 
[48] E. Voges and M. Hoffmann, “Optical waveguides on silicon combined with micromechanical structures”, LEOS Summer Topical Meeting Advanced Applications of Lasers In Materials and Processing Proceedings, pp. 69-70, 1996.

[49] Y. Shani, C. H. Henry, R. C. Kistler, R. F. Kazarinov, an K. J. Orlowsky, “Integrated optic adiabatic devices on silicon", IEEE Journal of Quantum Electronics, Vol. 27, No. 3, pp. 556-566, March 1991.

[50] J. R. Kulish, H. Franke, A. Singh, R. A. Lessard, and E. J. Knystautas, "Ion implantation, a method for fabricating light guides in polymers", Journal of Applied Physics, vol. 63, pp. 2517-2521, April 1988.

[51] A. J. Beuhler, D. A. Wargowski, K. D. Singer, and T. Kowalczyk, "Fabrication of low loss polyimide optical waveguides using thin-film multichip module process technology", in ProceedingsElectronic Components and Technology Conference, pp. 618-620. IEEE, May 1994.

[52] H. Franke and J. D. Crow, “Optical waveguiding in polyimide”, in Integrated Optical Circuit Engineering III. SPIE- The International Society for Optical Engineers, 1986.

[53] L. A. Hornak, S. K. Tewksbury, T. W. Weidman, E. W. Kwock, W. R. Holland, and G. L. Wolk, "The impact of polymer integrated optics on silicon wafer area networks", in Proceedings SPIE, Vol. 1337, pp. 12-22, 1990.

[54] A. J. Buehler and D. A. Wargowski, "Optical polyimides for single mode waveguides”, in Optoelectronic Interconnects, pp. 92-103. SPIE-The International Soceity for Optical Engineers, 1993. 
[55] S. M. Sze, VLSI Technology, Mcgraw-Hill, New York, second edition, 1988.

[56] K. S. Brown , Characterization of Polyphenylsilsesquioxane (PPSQ) as an Integrated Optical Waveguide Material, Masters thesis, West Virginia University, Morgantown, December 1996.

[57] AMOCO Chemical Company, Naperville, Illinois, Ultradel Microelectronic Coatings.

[58] OptiWave Corporation, Quebec, BPM_CAD 3.0 Waveguide Optics Modeling Software System.

[59] E. Hecht, Optics, Addison-Wesley, Reading, Massachusetts, second edition, 1987.

[60] Oxford Instruments, Reactive Ion Etching Process Flowsheets. 


\author{
Kolin S. Brown \\ 114 Country Squire \\ Morgantown, WV 26508 \\ (304)594-2893 \\ kbrown@michaelangelo.net
}

\title{
Education
}

\section{Ph.D. Engineering}

West Virginia University (Fall 2000)

Dissertation: On the Feasibility of Integrated Optical Waveguide-based In-Situ Monitoring of Microelectromechanical Systems (MEMS)

Specialization: Photonic and Microelectromechanical Systems (MEMS)

\section{Graduate Certificate in Materials Engineering}

College of Engineering and Mineral Resources, West Virginia University (Fall 1997)

Specialization: Photonic and Microelectronic Materials

\section{S. Electrical Engineering}

West Virginia University (Fall 1996)

Thesis: Characterization of Poly(phenylsilsesquioxane)(PPSQ) as an Integrated Optical

Waveguide Material.

Specialization: Photonics and Microelectronics.

\section{B. S. Electrical Engineering}

West Virginia University (Spring 1993)

\section{Employment:}

(August 2000-Present) Freshman Engineering Coordinator. College of Engineering and Mineral Resources, WVU. Responsibilities: I oversaw the freshman curriculum, classes, advising, and activities of engineering freshman students.

(August 1999-May 2000) Lecturer. Department of Electrical Engineering and Computer Science, WVU. Responsibilities: I was the CS5 Co-ordinator and my duties included training and overseeing 20 TAs a semester, as they taught introductory computer skills and Microsoft Office, and handling all conflicts in the CS5 Sections. I also taught EE 101 and EE103, where I taught introductory electrical engineering to mechanical, aerospace, industrial, civil, and petroleum engineering students.

(May1999-June1999) Research Assistant/ Lab Manager. Department of Electrical Engineering and Computer Science, WVU. Responsibilities: I am responsible for installing and characterizing a new 
Chemical Vapor Deposition unit in the micro fabrication lab at West Virginia University. I am also responsible for overseeing other graduate students using the lab and training the new Post Doctoral Employee on lab equipment operation.

(January 1999-May 1999) Teaching Assistant. Department of Electrical Engineering and Computer Science, WVU. Responsibilities: I taught EE101, an introductory electrical engineering course for mechanical, industrial and petroleum engineers. The class consisted of 51 juniors and seniors. Topics covered were introduction to DC and AC circuits and power machines.

(June 1994-December 1998) Research Assistant/ Lab Manager. Department of Electrical and Computer Engineering, WVU. Responsibilities: I was the sole student responsible fore the activation of the micro fabrication laboratory at West Virginia University. Responsibilities also included characterization of processes, equipment repair, and the fabrication and safety instruction of laboratory users.

(Sept. 1993- May 1994) Teaching Assistant. Department of Electrical and Computer Engineering, WVU. Sponsored by University Space Research Association. Responsibilities: I managed three NASA sponsored senior design seminar projects. (See above).

(Summer 1993) Summer Research Student. Goddard Flight Center, Sponsored by University Space Research Association. Responsibilities: I created a virtual model of the Intelligent Robotics Lab at Goddard by simulating robotic arms, tools, and lab environment with I-Grip Cad software. This project was part of a study for robotic arm assistance to astronauts during space flight repair to the Hubble telescope.

\section{Research:}

Co-Integration of Polymer Waveguides with Microelectromechanical Structures (MEMS) for Integrated Optical Metrology

(Doctoral Dissertation Research)

I am currently developing the micro fabrication process to integrate optical polymer waveguides with microelectromechanical structures to detect submicron motion. MEMS are movable silicon structures that are currently being used as sensors and actuators, however the submicron motion of these advanced devices is difficult to detect by standard optical microscopy. My research intends to expand the characterization of these devices by incorporating on-chip optical measurement techniques.

Sponsored by NSF.

\section{Characterization of Poly(phenylsilsesquioxane)(PPSQ) as an Integrated Optical Waveguide}

\section{Material}

(Master's Thesis Research)

I optically characterized PPSQ, a siloxane based polymer, for use in fabricating integrated waveguides for interconnections in hybrid optical-microelectronic systems. PPSQ has been explored for 
use as photoresist and as a dielectric material in microelectronic circuits. My research shows that PPSQ has low optical losses and a good index of refraction, making it a potential candidate material for optical interconnects.

Sponsored by NSF.

Demonstration Model of a Magnetic Levitation Transportation System

(Senior Design Project)

The Magnetic Levitation Model project was the first of a series of mag-lev senior projects at WVU. Our system was partially successful in demonstrating the independent magnetic forces required for mag-lev transportation: lift, guidance, and propulsion. The project demonstrated that a repulsive lift design requires a large guidance force which was only achievable with a mechanical guidance system. Sponsored by Dr. Ron Klein and the Department of Electrical and Computer Engineering at West Virginia University.

\section{Supervision of Undergraduate Research:}

Development and Fabrication of Microelectromechanical (MEMS) Optical Switches

As lab manager, I instructed and supervised three seniors in micro fabrication processing and safety as part of a senior design project. The students designed electrostatically actuated cantilever beams for laser beam steering, which were fabricated on 2 " silicon wafers. The fabrication methods included photolithography, wet chemical etching, and sputtering. The senior students were successful in creating 6 um cantilever beams which could be deflected with an applied voltage.

Sponsored by Dr. Larry Hornak and the Microelectronic Systems Research Center at West Virginia University.

\section{Capaciflector Imager for Virtual Robotic Arm Vision System}

As project Advisor, I managed three senior design groups, containing 11 students, working on the NASA sponsored projects. The groups focused on hardware design, noise reduction, and software interfacing of a capaciflector imager array for use as a vision system for remote robotic arm navigation in space. The capaciflector is a distance sensor invented by John Vranish of NASA and Robert McConnell of WVU for robotic arm positioning. The senior design groups were successful in developing the three subsystems, however total system integration was unsuccessful before the project deadline due to failure of the microprocessor.

Sponsored by University Space Research Associates and NASA.

\section{Publications:}

1. J. M. Dawson, K. S. Brown, P. F. Famouri, and L. A. Hornak, Through-Wafer Interrogation of Microstructure Motion for MEMS Feedback Control, Micromachining and Microfabrication Symposium, Miniaturized Systems with Micro-Optics and Micromechanics, (Santa Clara, CA September 1999, Accepted) 
2. F. M. Dickey, S. C. Holswade, L. A. Hornak, and K. S. Brown, Optical Methods for Micromachine Monitoring and Feedback, Sensors and Actuators A: Physical, (Printing Date Pending)

3. K. S. Brown, B. J. Taylor, J. M. Dawson, and L. A. Hornak, Polymer Waveguide Cointegration with Microelectromechanical Systems (MEMS) for Integrated Optical Metrology, SPIE Photonics West Symposium, Miniaturized Systems with Micro-Optics and Micromechanics III, pp. 112-122, (San Jose, CA March 1998)

4. K. S. Brown, B. J. Taylor, L. A. Hornak, and T. W. Weidman, Characterization of Poly(phenylsilsesquioxane) Thin-film Planar Optical Waveguides, IEEE Photonic Technology Letters, vol. 9(6), pp. 791-793 (June 1997)

5. K. S. Brown, B. J. Taylor, L. A. Hornak, and T. W. Weidman, Characterization of Poly(phenylsilsesquioxan)(PPSQ) for Planar Integrated Optical Waveguide Applications, SPIE Photonics West Symposium, Optical Interconnects and Packaging IV, pp. 163-169, (San Jose, CA February 1997).

6. L. A. Hornak, K. S. Brown, B. J. Taylor, and J. C. Barr, Polymer Guided-Wave Integrated Optics: An Enabling Technology for Micro-Opto-Electro-Mechanical Systems, SPIE Photonics West Symposium, Micro-Optics and Micromechanics II, pp. 124-135, (San Jose, Ca February 1997).

7. L. A. Hornak, J. C. Barr, W. D. Cox, K. S. Brown, R. A. Morgan, and M. K. Hibbs-Brenner, Low Temperature (10-300K) Characterization of MOVPE-grown Vertical-Cavity Surface-Emitting Lasers, IEEE Photonics Technology Letters, vol. 7(10), pp. 110-1112 (1995).

8. L. A. Hornak, S. K. Tewksbury, J. C. Barr, W. D. Cox, and K. S. Brown, Optical Interconnections and Cryoelectronics: Complimentary Enabling Technologies for Emerging Mainstream Systems, SPIE Photonics West Symposium, Optical Interconnections III, pp. 224-234, (San Jose, CA February 1995).

9. J. C. Barr, W. D. Cox, J. J. Wolfgang, L. A. Hornak, and K. S. Brown, Development and Fabrication of Microelectromechancial Optical Switches, Proceedings of the Ninth National Conference on Undergraduate Research 1995, pp. 599-603, (Asheville, NC July 1995).

10. K. Brown, R. McConnell, et. al., Electric Camera Visual Imaging System, Proceedings of the Tenth Annual Summer Conference, NASA/USRA University Advanced Aeronautics Design Program and advanced Space Design Program, (Pasadena, CA June 1994). 
11. K. Brown, C. Harshbarger, and R. Martin, Design for a Model of a Magnetic Levitation Transportation System, Proceedings of the Southeast Regional Undergraduate Design '93, North Carolina State University Electrical and Computer Engineering Undergraduate Design Center, pp. 1-6, (Raleigh, NC April 1993).

\section{Presentations:}

"The Characterization of poly(phenylsilsesquioxane)(PPSQ) for use as an Integrated Optical Interconnection". Photonic Materials and Devices Seminar, Morgantown, WV, October 1995.

\section{Honors and Awards:}

\section{Phi Kappa Phi}

Mountain (WVU Ranking Honorary)(Treasurer 1994-1998)

Golden Key National Honor Society

Tau Beta Pi Engineering Honorary

Eta Kappa Nu Electrical Engineering Honorary (Recording Secretary 1992-93)

WVU Presidential Scholarship 1989-1993

Mr. Mountaineer 1992 